\title{
Case Studies \\ Assessment Report
}

\section{M.}

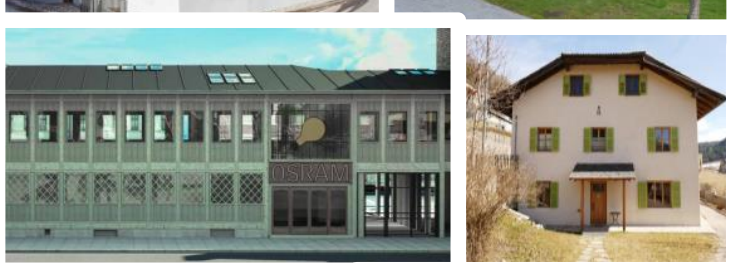

\section{.}
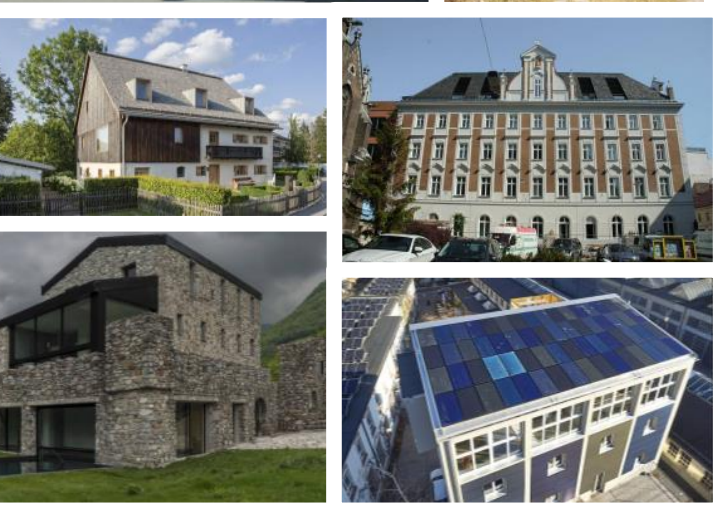
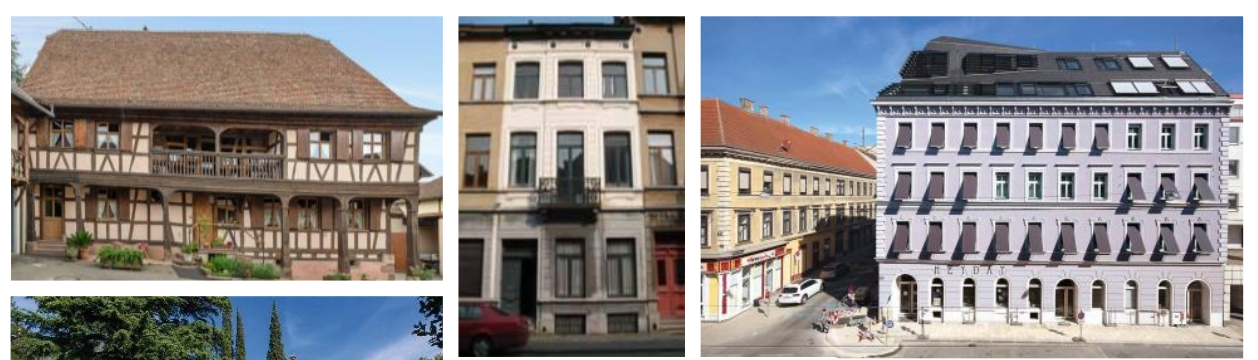

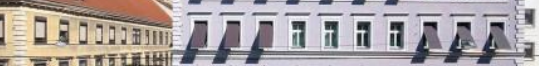

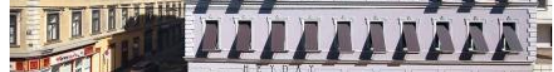
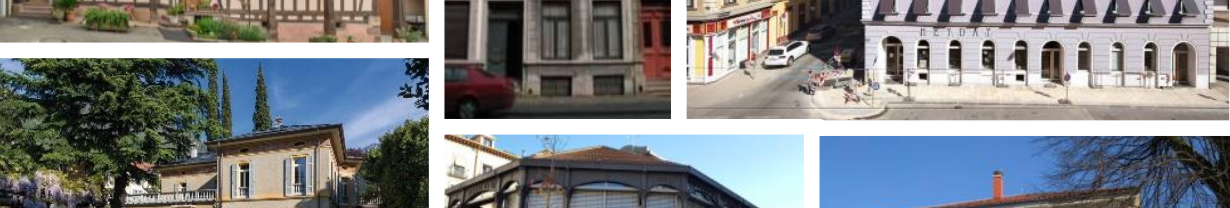

4.
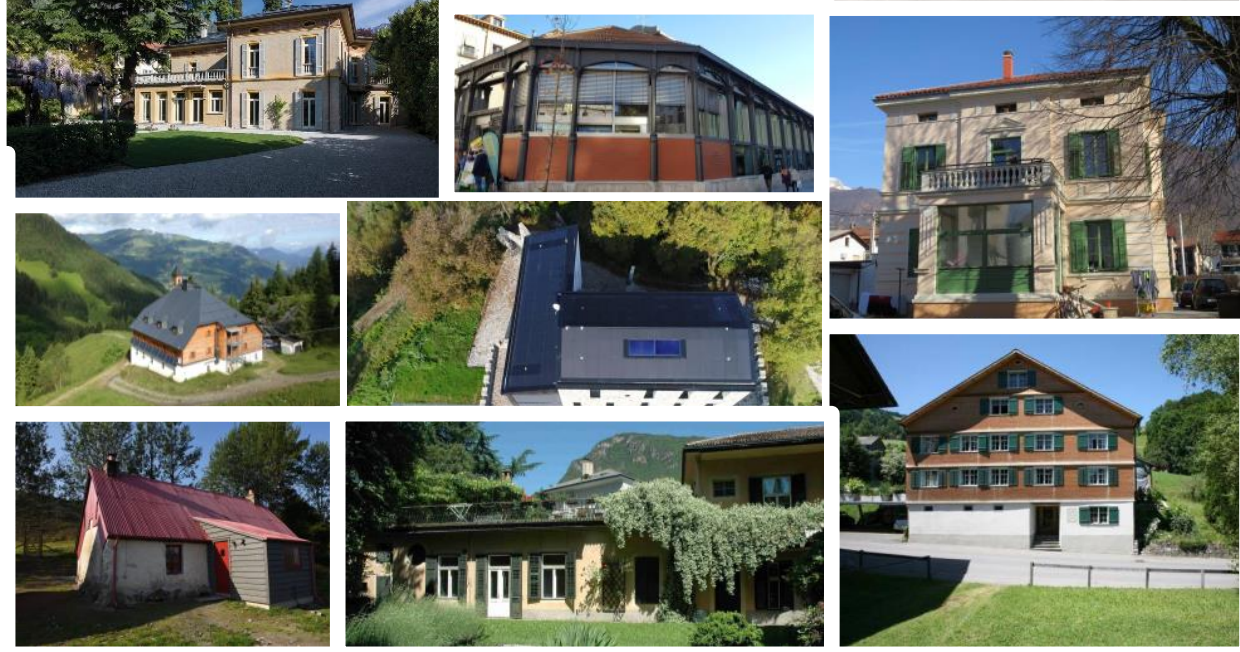

IEA SHC TASK 59 | EBC Annex 76

Renovating Historic Buildings Towards Zero Energy 


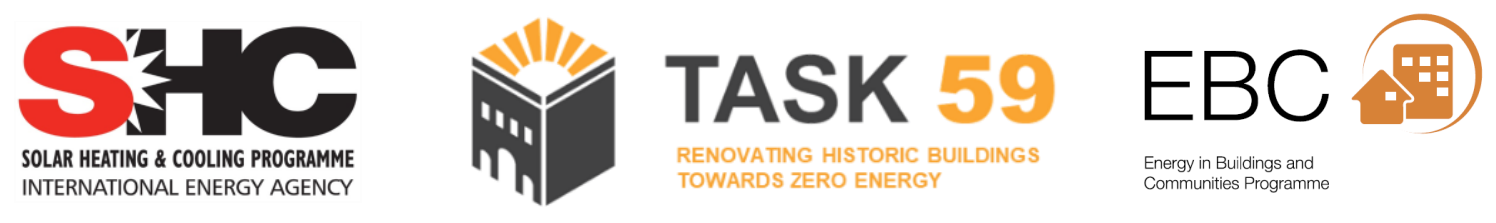

\section{Case Studies \\ Assessment Report}

\section{SHC Task 59 | EBC Annex 76 | Report D.A2}

Daniel Herrera, Franziska Haas, Alexandra Troi (EURAC Bolzano)

Gustaf Leijonhufvud, Tor Broström (Uppsala Universitet)

Alexander Rieser (University Innsbruck)

Jørgen Rose (Aalborg Universitet)

Walter Hüttler, Susanne Kuchar (e7 energy innovation \& engineering, Vienna)

October 2021

SHC Task 59 | EBC Annex 76 | Report D.A2

DOI: 10.18777/ieashc-task59-2021-0001

The contents of this report do not necessarily reflect the viewpoints or policies of the International Energy Agency (IEA) or its member countries, the IEA Solar Heating and Cooling Technology Collaboration Programme (SHC TCP) members or the participating researchers. 


\section{IEA SHC Task 59 | EBC Annex 76: Deep renovation of historic buildings towards lowest possible energy demand and $\mathrm{CO}_{2}$ emission (NZEB)}

\section{Solar Heating and Cooling Technology Collaboration Programme (IEA SHC)}

The Solar Heating and Cooling Technology Collaboration Programme was founded in 1977 as one of the first multilateral technology initiatives ("Implementing Agreements") of the International Energy Agency.

Our mission is "Through multi-disciplinary international collaborative research and knowledge exchange, as well as market and policy recommendations, the IEA SHC will work to increase the deployment rate of solar heating and cooling systems by breaking down the technical and non-technical barriers."

IEA SHC members carry out cooperative research, development, demonstrations, and exchanges of information through Tasks (projects) on solar heating and cooling components and systems and their application to advance the deployment and research and development activities in the field of solar heating and cooling.

Our focus areas, with the associated Tasks in parenthesis, include:

- $\quad$ Solar Space Heating and Water Heating (Tasks 14, 19, 26, 44, 54)

- Solar Cooling (Tasks 25, 38, 48, 53, 65)

- Solar Heat for Industrial and Agricultural Processes (Tasks 29, 33, 49, 62, 64)

- Solar District Heating (Tasks 7, 45, 55)

- Solar Buildings/Architecture/Urban Planning (Tasks 8, 11, 12, 13, 20, 22, 23, 28, 37, 40, 41, 47, 51, 52, 56, $59,63,66)$

- Solar Thermal \& PV (Tasks 16, 35, 60)

- Daylighting/Lighting (Tasks 21, 31, 50,61)

- Materials/Components for Solar Heating and Cooling (Tasks 2, 3, 6, 10, 18, 27, 39)

- Standards, Certification, and Test Methods (Tasks 14, 24, 34, 43, 57)

- $\quad$ Resource Assessment (Tasks 1, 4, 5, 9, 17, 36, 46)

- $\quad$ Storage of Solar Heat (Tasks 7, 32, 42, 58, 67)

In addition to our Task work, other activities of the IEA SHC include our:

$>$ SHC Solar Academy

$>$ Solar Heat Worldwide, annual statics report

$>$ SHC International Conference

Our members

$\begin{array}{lll}\text { Australia } & \text { European Copper Institute } & \text { SICREEE } \\ \text { Austria } & \text { France } & \text { Slovakia } \\ \text { Belgium } & \text { Germany } & \text { South Africa } \\ \text { Canada } & \text { International Solar Energy Society } & \text { Spain } \\ \text { CCREEE } & \text { Italy } & \text { Sweden } \\ \text { China } & \text { Netherlands } & \text { Switzerland } \\ \text { Denmark } & \text { Norway } & \text { Turkey } \\ \text { EACREEE } & \text { Portugal } & \text { United Kingdom } \\ \text { ECREEE } & \text { RCREEE } & \\ \text { European Commission } & \text { SACREEE } & \end{array}$

For more information on the IEA SHC work, including many free publications, please visit www.iea-shc.org.

\section{Energy in Buildings and Communities Technology Collaboration Programme (IEA EBC)}

To reach the objectives of SHC Task 59 the IEA SHC implementing Agreement has collaborated with the IEA EBC Implementing Agreement at a "Medium Level Collaboration", and with the IEA PVPS Implementing Agreement at a "Minimum Level Collaboration" as outlined in the SHC Implementing Agreement's Policy on Collaboration. 


\section{Contents}

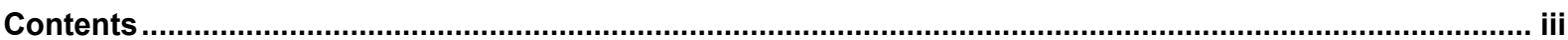

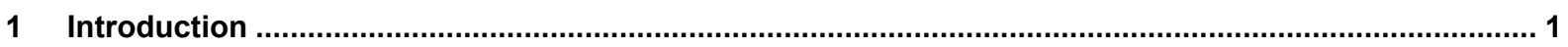

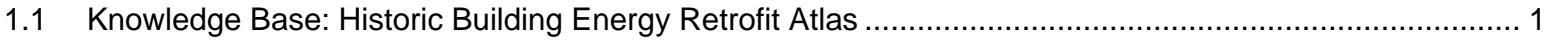

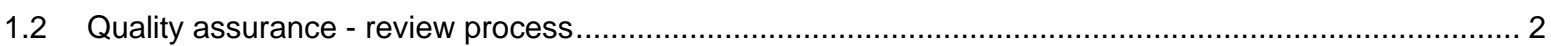

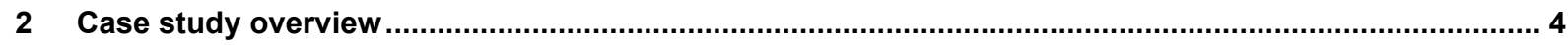

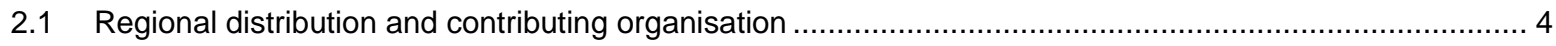

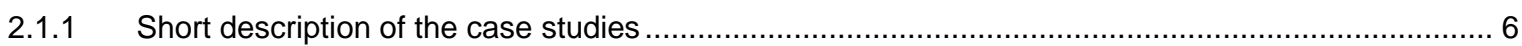

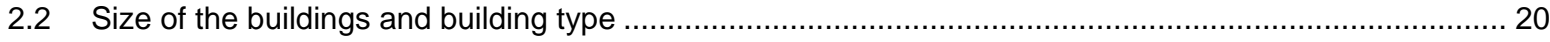

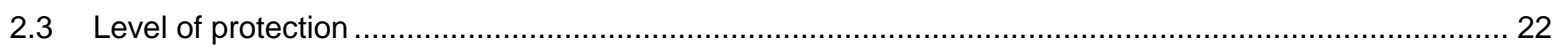

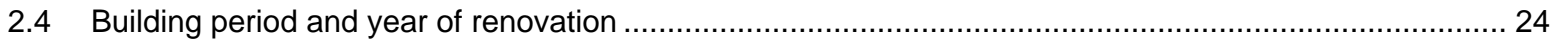

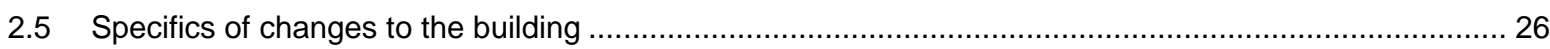

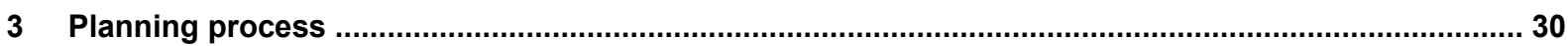

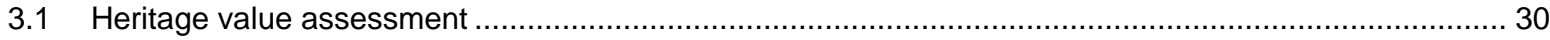

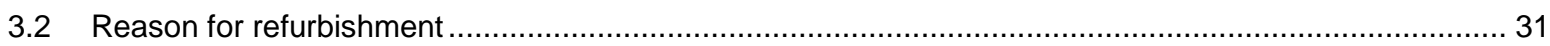

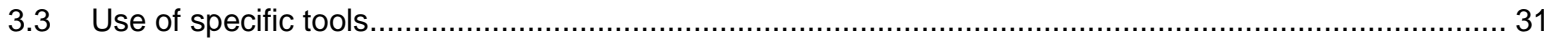

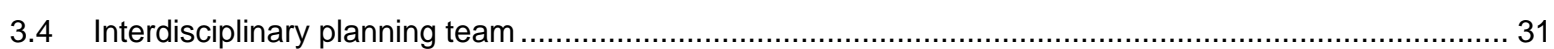

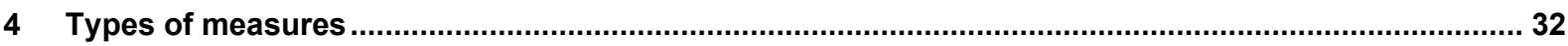

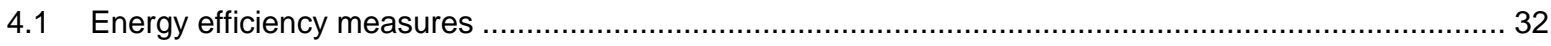

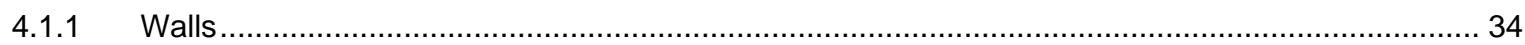

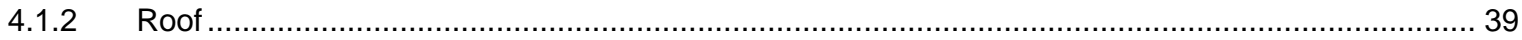

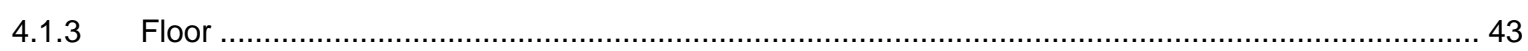

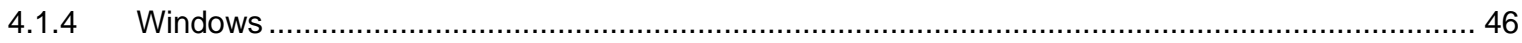

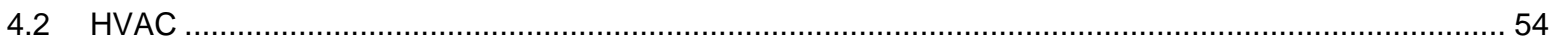

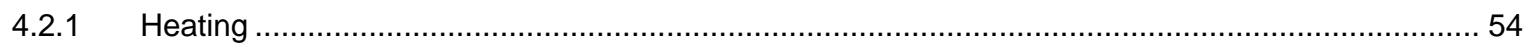

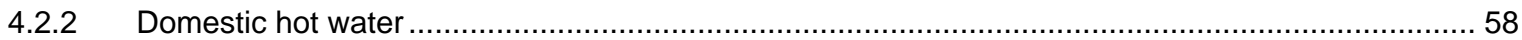

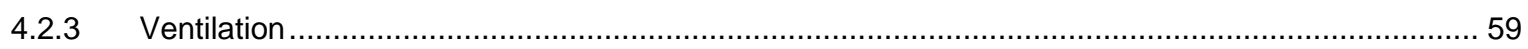

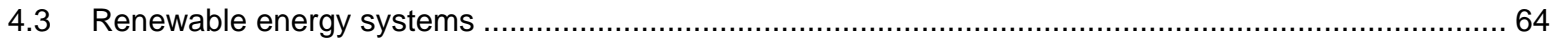

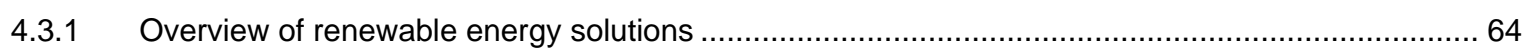

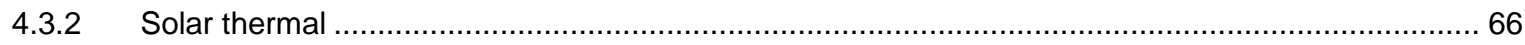

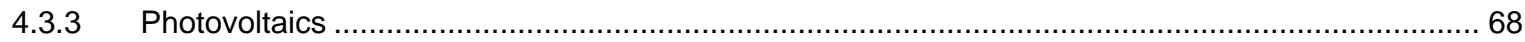

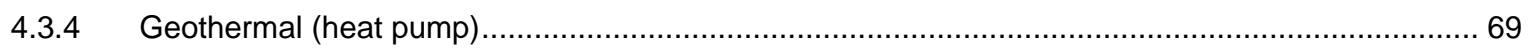

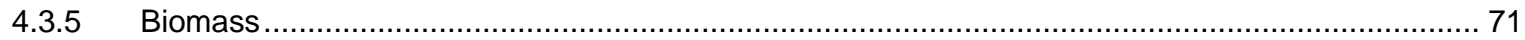

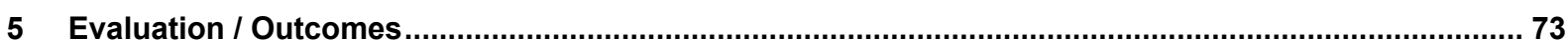

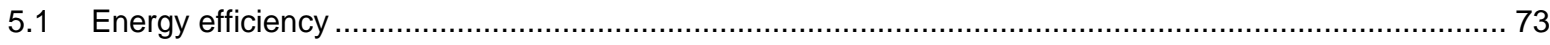

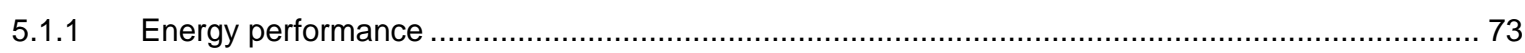

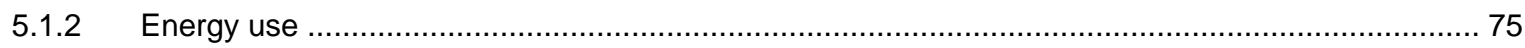

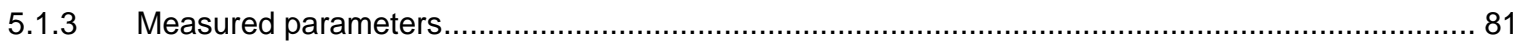

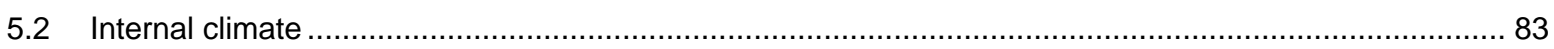

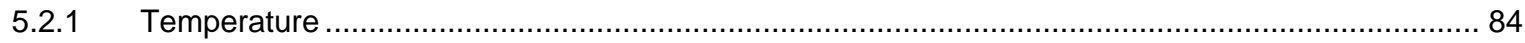




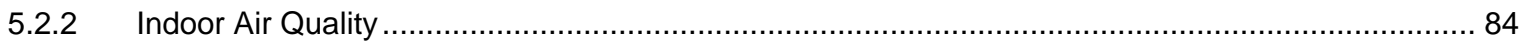

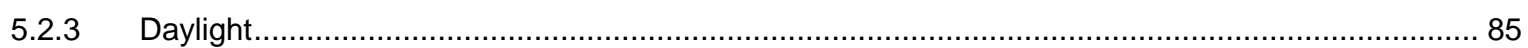

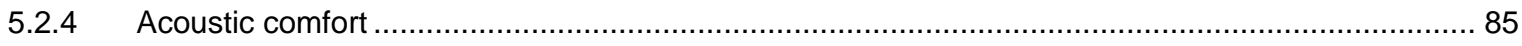

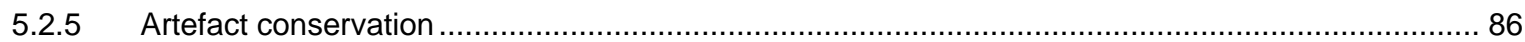

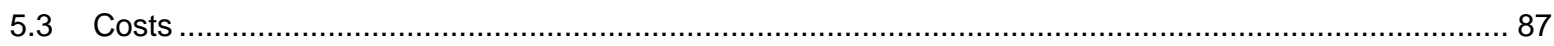

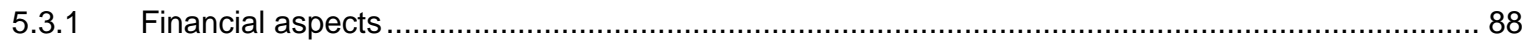

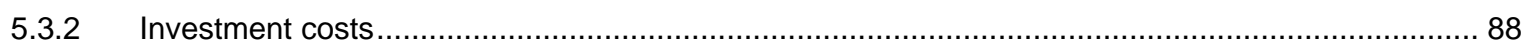

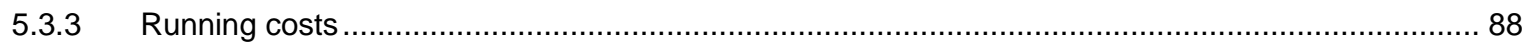

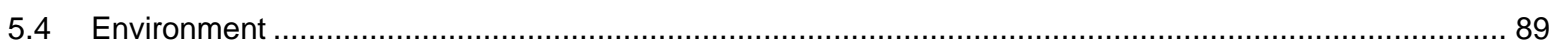

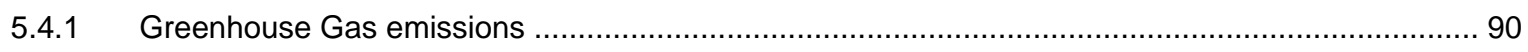

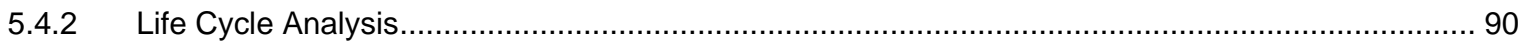

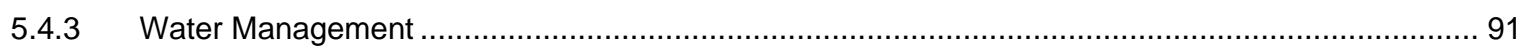

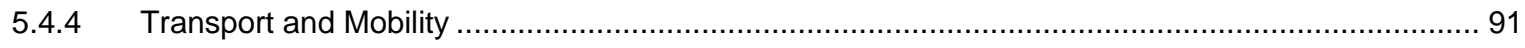

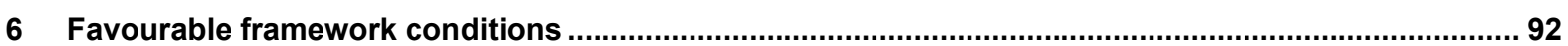

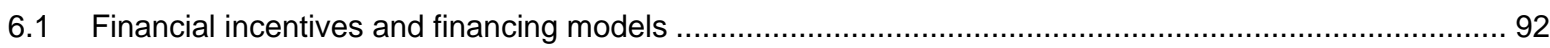

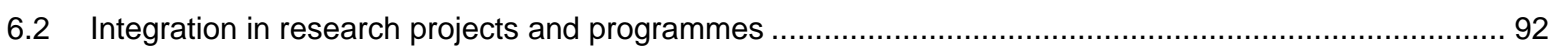

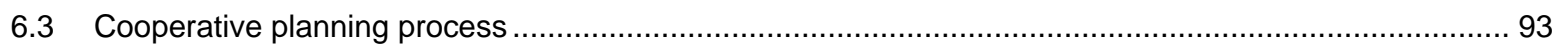

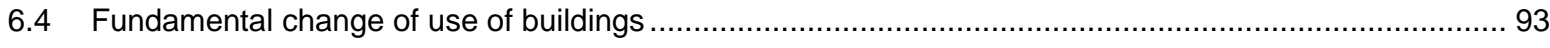

6.5 Other beneficial factors for good practice examples................................................................... 93

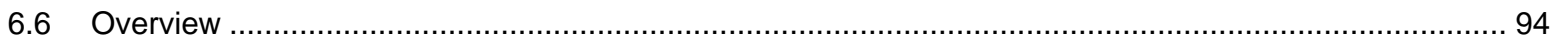

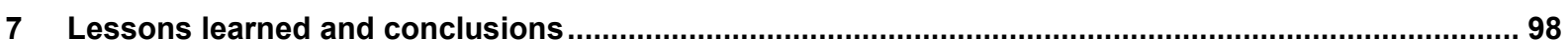

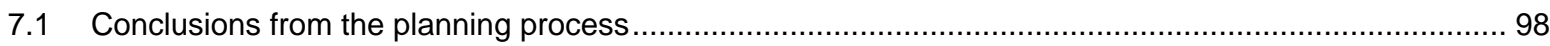

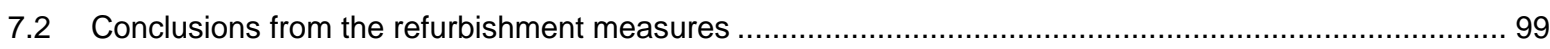

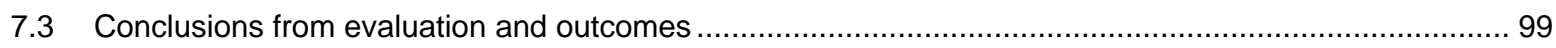

7.4 Conclusions regarding the favourable framework conditions and transferability ............................. 100 


\section{Introduction}

\subsection{Knowledge Base: Historic Building Energy Retrofit Atlas}

One of the main targets of SHC Task 59 is to provide a solid knowledge base on deep renovation of historic buildings. The Historic Building Energy Retrofit Atlas (HiBERatlas, www.hiberatlas.com) provides a bestpractice database of exemplary energy efficient interventions in historic buildings. The database presents bestpractice examples of how a historic building can be renovated to achieve high levels of energy efficiency while respecting and protecting its heritage significance.

The scope and content of this database has been discussed with the interdisciplinary panel of experts that forms the SHC Task 59. The aim is to make existing best-practice experiences available to decision-makers. However, this is not a homogeneous target group as it includes different stakeholders with different understanding of the complexity of the renovation process (e.g. building owners, real estate developers and managers, public sector, and architects, engineers and planners). It is crucial to identify the characteristics that define a best-practice as well as the requirements for the data provided so that the information made available to the users meets their needs.

Establishing a single quantitative criterion or threshold to measure the degree of success of an intervention exclusively as a function of the energy saving (i.e. $\mathrm{kWh} / \mathrm{m}^{2}$ ), would not be compatible with the definition of lowest possible energy demand presented before as it goes against the principle that every building must be considered individually. Instead, this database considers as best-practice any example that fulfils the following requirements:

- Renovation of the whole building. The database aims to present examples that have sought an improvement in the building's overall energy performance, rather than remain limited to the improvement of a single aspect of the building. Also, in the case of retrofitted historic buildings, the whole is often greater than the sum of its parts and the integrated application of solutions is key for the success of the intervention (Troi and Bastian, 2015) ${ }^{1}$.

- The project has been implemented. Most of the limitations in the renovations of historic buildings appear when it comes to the compatibility with the existing construction and use. It is therefore important that the database shows renovation projects that have already been implemented.

- The intervention followed the results of a thorough heritage value assessment. Energy improvement in historic buildings cannot be achieved at the expense of their heritage value. This database should therefore illustrate the relationship between the building's heritage significance and the solutions adopted.

- A significant energy demand reduction was achieved. Although the result achieved will depend largely on the heritage value assessment, all projects included in the database should have pursued an ambitious renovation in terms of efficiency.

- A detailed documentation of the decision process, technical solutions and evaluation results was made available. Access to targeted and robust information is crucial to the successful dissemination of best-practices (The Swedish Environmental Protection Agency, 2003)2.

The Historic Building Energy Retrofit Atlas is a joint effort from SHC Task 59 and the Interreg AlpineSpace project ATLAS and is available online since October 2019 (as a beta version) and currently undergoing final testing.

The contributions from partners of SHC Task 59 and the Interreg AlpineSpace project ATLAS are expected to add up to 80 case studies. Such a repository is expected to attract the interest of decision-makers and additionally create the momentum needed to attract new examples from architects, owners or new research projects.

\footnotetext{
1 Troi, A. and Bastian, Z. (2015), Energy Efficiency Solutions for Historic Buildings: A Handbook, BIRKHÄUSER, Basel, available at: https://doi.org/10.1017/CBO9781107415324.004.

2 The Swedish Environmental Protection Agency. (2003), The Power of Example: An Evaluation of How Examples of Best Practices, Good Examples and Success Stories Are Used in the Work for Sustainability, Naturvårdsverket, Stockholm.
} 


\subsection{Quality assurance - review process}

Ensuring the quality of the best-practices displayed in the database is crucial to help eradicating any concern about professionals' expertise. The implementation of a review process that can assess the validity of the projects and, most importantly, the way they are documented becomes necessary. The ultimate goal of the review process is not to reject proposed examples but to ensure their robustness and to improve the way they are presented. The review model takes inspiration from the academic peer-review process. Every best-practice gathered during the project was and will be assessed by the experts participating in the SHC Task 59 following this methodology to test the feasibility of such review process. At the end of the SHC Task 59 project, the lessons learned will be used to adapt and improve the reviewing model.

Figure 1: Review process in SHC Task 59

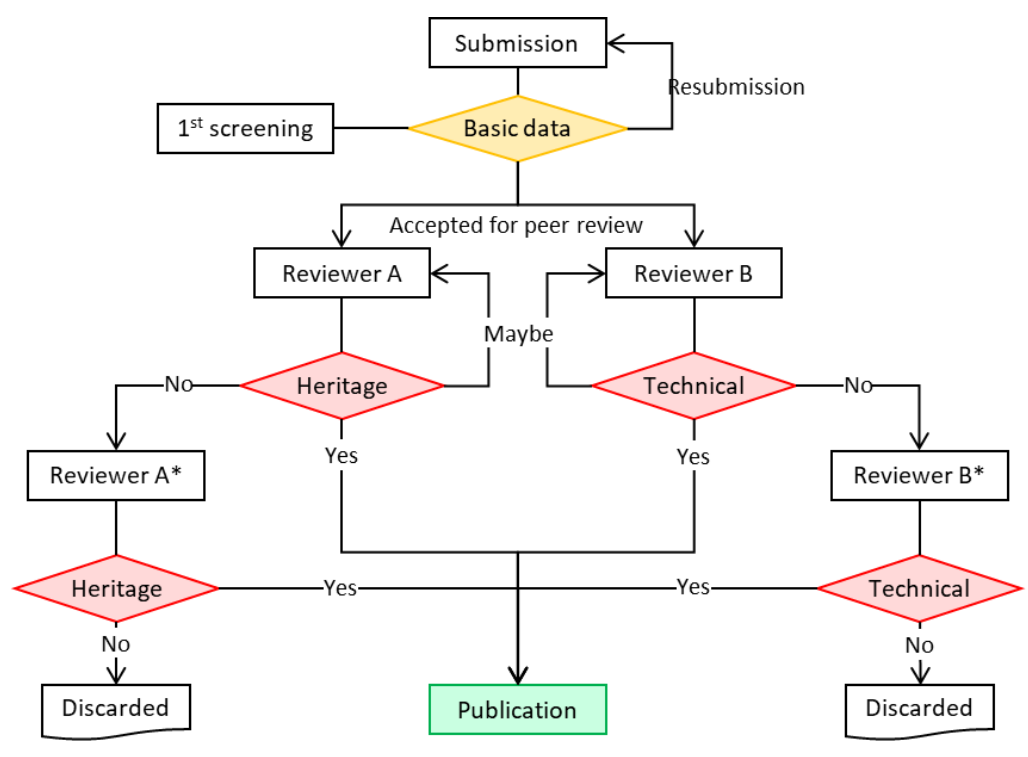

After checking the completeness of the information provided, the best-practice is assigned to at least two members with different expertise: Heritage or Technical. If a case study is rejected by one of the reviewers, this is submitted to an alternative reviewer for a second opinion. A second rejection will mean that the project should not be included in the database.

For the review a template was developed, that was tailored to heritage and technical focus. It was asked for an overall evaluation according to Heritage Compatibility and Technical feasibility. Furthermore, there is the possibility to mention other positive aspects of the project, like economic and environmental aspects, robustness and others.

Table 1 Extract of the review template, first part with key information

1.) Overall evaluation (the overall evaluation should be completed for all aspects 1.1 - 1.3 by all reviewers regardless of their expertise):

1.1) What is your overall assessment of the project regarding Heritage Compatibility? Please select one of the categories below and write a short explanation

\begin{tabular}{|c|c|c|c|}
\hline $\begin{array}{c}\text { Recommended } \\
\text { as role model }\end{array}$ & $\begin{array}{c}\text { Recommended } \\
\text { with limitations }\end{array}$ & $\begin{array}{c}\text { Not } \\
\text { recommended }\end{array}$ & $\begin{array}{l}\text { Please justify your selection shortly! Further explanations } \\
\text { can be found below. }\end{array}$ \\
\hline & & &
\end{tabular}

This key- information part of the template is followed by questions which are supposed to be answered more in detail, asking mainly for the completeness and comprehensibility of the documentations.

It turned out that most projects were rated yellow, what means the projects seemed suitable for the database, but the documentation had to be at least partially completed. Only one project received a red rating in the first review 
from the Heritage expert. After a second review, however, this project was also given a yellow rating and asked to provide additional information. Only one of the evaluated projects received a green rating in both aspects, heritage and technical, with the first review.

Table 2 Overview of the review results (exemplary)

\begin{tabular}{|c|c|c|c|}
\hline & & Heritage Compatibility & Technical Feasibility \\
\hline \multirow[t]{3}{*}{ Bauernhof Trins } & Heritage Compatibility & $\bar{S}$ & \\
\hline & Technical Feasibility & & \\
\hline & Other aspects (economic, environmental, social,...) & & \\
\hline \multirow[t]{3}{*}{ Beim Jäger } & Heritage Compatibility & still missing & \\
\hline & Technical Feasibility & & \\
\hline & Other aspects (economic, environmental, social,...) & & \\
\hline \multirow[t]{3}{*}{ Hof 6, Schwarzenberg, Voralberg, Austria } & Heritage Compatibility & & \\
\hline & Technical Feasibility & & \\
\hline & Other aspects (economic, environmental, social,...) & & \\
\hline \multirow[t]{3}{*}{ Notarjeva vila } & Heritage Compatibility & 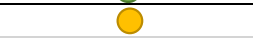 & still missing \\
\hline & Technical Feasibility & & \\
\hline & Other aspects (economic, environmental, social,...) & & \\
\hline \multirow[t]{3}{*}{ Rainhof } & Heritage Compatibility & 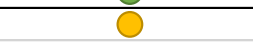 & \\
\hline & Technical Feasibility & ) & \\
\hline & Other aspects (economic, environmental, social,...) & S & \\
\hline \multirow[t]{4}{*}{ Solar silo } & Heritage Compatibility & still missing & \\
\hline & Technical Feasibility & & \\
\hline & Other aspects (economic, environmental, social,...) & & \\
\hline & Solutions & & \\
\hline \multirow[t]{5}{*}{ Villa Castelli } & Heritage Compatibility & & \\
\hline & Technical Feasibility & 0 & \\
\hline & Other aspects (economic, environmental, social,...) & 0 & P \\
\hline & & & \\
\hline & & Heritage Compatibility & Technical Feasibility \\
\hline \multirow[t]{3}{*}{ Klitgaarden } & Heritage Compatibility & 00 & \\
\hline & Technical Feasibility & $\bigcirc$ & \\
\hline & Other aspects (economic, environmental, social,...) & O & \\
\hline \multirow[t]{3}{*}{ Maison Rubens } & Heritage Compatibility & & \\
\hline & Technical Feasibility & & \\
\hline & Other aspects (economic, environmental, social,...) & & \\
\hline \multirow[t]{3}{*}{ Osramhuset } & Heritage Compatibility & & \\
\hline & Technical Feasibility & & \\
\hline & Other aspects (economic, environmental, social,...) & & \\
\hline \multirow[t]{3}{*}{ Maison Rubens } & Heritage Compatibility & & \\
\hline & Technical Feasibility & & \\
\hline & Other aspects (economic, environmental, social,...) & & \\
\hline \multirow[t]{3}{*}{ Half-timberframed house } & Heritage Compatibility & 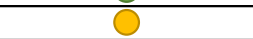 & $J$ \\
\hline & Technical Feasibility & & \\
\hline & Other aspects (economic, environmental, social,...) & O & 0 \\
\hline \multirow{3}{*}{ Mercado del Val } & Heritage Compatibility & & O \\
\hline & Technical Feasibility & & 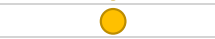 \\
\hline & Other aspects (economic, environmental, social,...) & 0 & 0 \\
\hline
\end{tabular}




\section{Case study overview}

This case study assessment report covers 69 case studies $^{3}$. They can be categorized according to regional distribution, size, type of building, level of protection, age and year of renovation. Below, the buildings are presented in these categories.

\subsection{Regional distribution and contributing organisation}

The case studies were collected in 12 countries and are located in Austria, Belgium, Switzerland, Denmark, France, Italy, Slovenia, Spain, Turkey, the United Kingdom, and the USA.

The organisations responsible for the case studies are e7, UIBK and EIV in Austria, BBRI/UCL/FHA in Belgium, SUPSI in Switzerland, FraunhoferISE and EURAC in Germany, SBi in Denmark, Cerema in France, EURAC, POLIMI, Arch. Mayr Fingerle/Truden and UNIGE in Italy, PRC in Slovenia, CARTIF and TECNALIA in Spain, IYTE in Turkey, HES in the UK and Drexel University in the USA (see table below).

Table 3 - Overview of the case studies, their location, the contributing person/organisation

\begin{tabular}{|c|c|c|c|}
\hline No & Case study & Country & Organisation \\
\hline 1 & Klostergebäude Kaiserstrasse & AT & e7 \\
\hline 2 & Farm house Trins & AT & UIBK \\
\hline 3 & Hof 6, Schwarzenberg * & AT & EIV \\
\hline 4 & Kelchalm - Bochumer alpine hut & AT & UIBK \\
\hline 5 & Mariahilferstrasse 182 & AT & e7 \\
\hline 6 & House Maurer, Wolfurt * & AT & EIV \\
\hline 7 & House Breuer, Tschagguns * & AT & EIV \\
\hline 8 & Music school in Velden & AT & e7 \\
\hline 9 & Hof Neuhäusl & AT & UIBK \\
\hline 10 & Community Hall Zwischenwasser * & AT & EIV \\
\hline 11 & Freihof Sulz * & AT & EIV \\
\hline 12 & Oeconomy building Josef Weiss * & AT & EIV \\
\hline 13 & Giatla Haus & AT & UIBK \\
\hline 14 & Rhine Valley House Irgang * & AT & EIV \\
\hline 15 & Baur Residence, Lustenau * & AT & EIV \\
\hline 16 & Kasperhof & AT & UIBK \\
\hline 17 & Maison Rubens & $\mathrm{BE}$ & BBRI/UCL/FHA \\
\hline 18 & Half-timberframed house in Alken & $\mathrm{BE}$ & BBRI/UCL/FHA \\
\hline 19 & Doragno Castle, Rovio & $\mathrm{CH}$ & SUPSI \\
\hline 20 & Solar silo & $\mathrm{CH}$ & SUPSI \\
\hline 21 & Wohn- und Geschäftshaus Feldbergstrasse & $\mathrm{CH}$ & SUPSI \\
\hline 22 & Mehrfamilienhaus Magnusstrasse & $\mathrm{CH}$ & SUPSI \\
\hline 23 & St. Franziskus Church Ebmatingen & $\mathrm{CH}$ & SUPSI \\
\hline 24 & Kindergarten and apartments (PEB) Chur & $\mathrm{CH}$ & SUPSI \\
\hline 25 & Single family home Luisenstrasse - Bern & $\mathrm{CH}$ & SUPSI \\
\hline 26 & Single Family House - Gstaad & $\mathrm{CH}$ & SUPSI \\
\hline 27 & Glaserhaus in Affoltern & $\mathrm{CH}$ & SUPSI \\
\hline 28 & PalaCinema Locarno & $\mathrm{CH}$ & SUPSI \\
\hline 29 & Casa Rossa Chemnitz & $\mathrm{DE}$ & FraunhoferISE \\
\hline 30 & Rathaus Bergrheinfeld & $\mathrm{DE}$ & EURAC \\
\hline
\end{tabular}

\footnotetext{
${ }^{3}$ Some organisations, who were contributing to the case studies, were involved in SHC Task 59 and the ATLAS project.
} Therefore, projects from partners only working in Atlas are marked with * in Table 3. 


\begin{tabular}{|c|c|c|c|}
\hline 31 & Farmhouse Straub & DE & EURAC \\
\hline 32 & Early work Sep Ruf & DE & EURAC \\
\hline 33 & Ackerbürgerhäuschen & DE & EURAC \\
\hline 34 & Ritterhof & DE & EURAC \\
\hline 35 & Rathaus Burgkunstadt & DE & EURAC \\
\hline 36 & Osramhuset & DK & $\mathrm{SBi}$ \\
\hline 37 & Klitgaarden & DK & $\mathrm{SBi}$ \\
\hline 38 & RYESGADE 30 & DK & $\mathrm{SBi}$ \\
\hline 39 & Timber-framed house in Alsace & FR & Cerema \\
\hline 40 & Elementary School in Mulhouse & FR & Cerema \\
\hline 41 & Timber-framed barn in the north of France & FR & Cerema \\
\hline 42 & Rainhof & IT & EURAC \\
\hline 43 & Villa Castelli & IT & EURAC \\
\hline 44 & Ansitz Kofler & IT & EURAC \\
\hline 45 & Basilica di Santa Maria di Collemaggio & IT & POLIMI \\
\hline 46 & House Pernter * & IT & Arch. Mayr Fingerle/Truden \\
\hline 47 & Kohlerhaus & IT & EURAC \\
\hline 48 & Ruckenzaunerhof & IT & EURAC \\
\hline 49 & Aussergrubhof & IT & EURAC \\
\hline 50 & Oberbergerhof & IT & EURAC \\
\hline 51 & Platzbonhof & IT & EURAC \\
\hline 52 & Mairhof & IT & EURAC \\
\hline 53 & Obergasserhof & IT & EURAC \\
\hline 54 & Rebecco Farm & IT & PoliMi \\
\hline 55 & Villa Capodivacca & IT & UNIGE \\
\hline 56 & House Moroder & IT & EURAC \\
\hline 57 & Huberhof & IT & EURAC \\
\hline 58 & Notarjeva vila * & SL & PRC \\
\hline 59 & Rožna ulica 15, Idrija * & SL & PRC \\
\hline 60 & Hiša trentarskih vodnikov * & SL & PRC \\
\hline 61 & Idrija mercury smelting plant * & SL & PRC \\
\hline 62 & Mercado del Val, Valladolid & SP & CARTIF \\
\hline 63 & Correria 119 & SP & Tecnalia \\
\hline 64 & Ahmet Aga Mansion & TK & IYTE \\
\hline 65 & Nwcip Pasa Library & TK & IYTE \\
\hline 66 & Downie's Cottage & UK & HES \\
\hline 67 & Hollyrood Park Lodge & UK & HES \\
\hline 68 & Annat Road & UK & HES \\
\hline 69 & Aspinall Courthouse & USA & Drexel University \\
\hline
\end{tabular}




\subsubsection{Short description of the case studies}

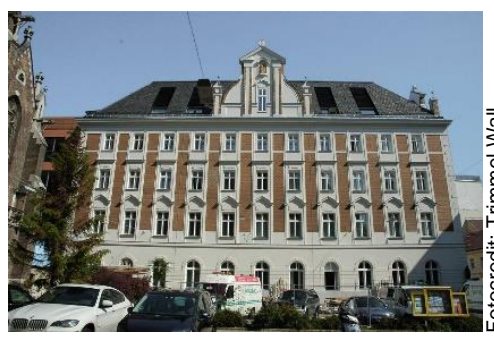

A multi-purpose used convent building in the heart of Vienna has been refurbished with particular attention to monument preservation and to a new solution for renovating Viennese-type box windows. The goal was to present a sustainable system solution with the energetic refurbishment of the existing building and the highly efficient loft conversion according to the requirements of the monument protection.

\begin{tabular}{|llll|}
\hline $\begin{array}{l}\text { Klostergebäude Kaiserstrasse } \\
\text { Vienna, AT }\end{array}$ & $\begin{array}{l}\text { Building Period: } 1850-1899 \\
\text { Renovation: } 2013\end{array}$ & $\begin{array}{l}\text { Use: Residential (urban) } \\
\text { Protection level: listed }\end{array}$ & $\begin{array}{l}\text { Contact: } \\
\text { e7 energy innovation \& engineering }\end{array}$ \\
\hline
\end{tabular}

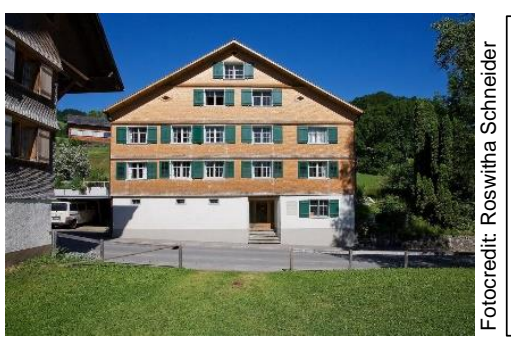

The former farm house and later on home of the painter Angelika Kaufmann was almost 450 years old, when the architect and new owner Thomas Mennel decided to restore the building and play with it's given qualities. He kept the outward appearance and changed its internals into a spaceship full of places to experience, with its different light and shades - it is a playground and an oasis in the same.

\begin{tabular}{|llll|}
\hline $\begin{array}{l}\text { Hof 6, Schwarzenberg } \\
\text { Vorarlberg, AT }\end{array}$ & $\begin{array}{l}\text { Building Period: } 1600-1700 \\
\text { Renovation: 2013 }\end{array}$ & $\begin{array}{l}\text { Use: Residential (rural) } \\
\text { Protection level: listed }\end{array}$ & $\begin{array}{l}\text { Contact: } \\
\text { Energieinstitut Vorarlberg }\end{array}$ \\
\hline
\end{tabular}

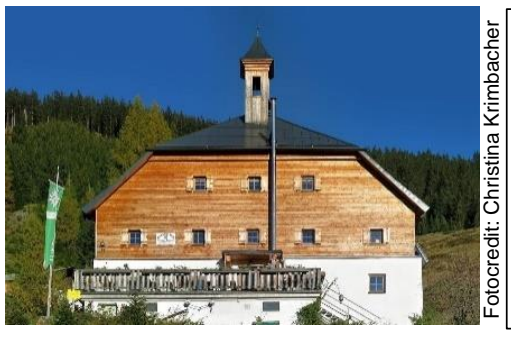

Exemplary sustainable renovation of a historic mountain hut at 1,432 meters above sea level in Aurach near Kitzbühel. The renovation resulted in a significant increase in the levels of comfort in a mountain hut exposed to severe weather conditions. The building is used all year round after renovation.

\begin{tabular}{llll}
$\begin{array}{l}\text { Kelchalm - Bochumer Hütte } \\
\text { Tirol, AT }\end{array}$ & Building Period: $1800-1849$ & Use: Hotel/Restaurant & Contact: \\
Renovation: 2013 & Protection level: not listed & University of Innsbruck \\
\hline
\end{tabular}

\begin{tabular}{|l|l|}
$\begin{array}{l}\text { Modernization of a Gründerzeit building with the use of an aerogel insulating plaster after } \\
\text { it was demolished by an gas explosion. In addition to the restored façade, the exterior of } \\
\text { the new attic storey is initially noticeable. he building received two major awards: the 1st } \\
\text { prize of the 33rd Vienna Urban Renewal Prize by the National Guild Construction Vienna, } \\
\text { and the State Prize for Architecture and Sustainability } 2019 \text { by the Federal Ministry for } \\
\text { Sustainability and Tourism. }\end{array}$ \\
\begin{tabular}{llll} 
Mariahilferstrasse 182 & Building Period: $1850-1899$ & $\begin{array}{l}\text { Use: Residential (urban) } \\
\text { Protection level: not listed }\end{array}$ & $\begin{array}{l}\text { Contact: } \\
\text { e7 energy innovation \& engineering }\end{array}$ \\
\hline
\end{tabular}
\end{tabular}

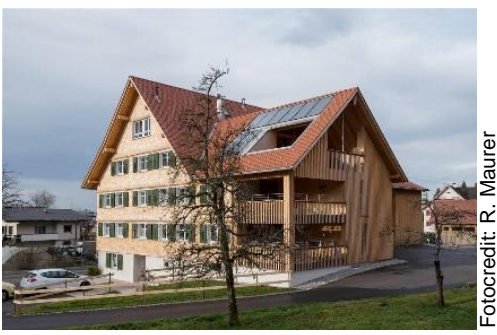

The owner managed to renovate the building in a way, which kept the outer appearance. The hole faced and situation of the roof were kept. The main house did not change much while the extension changes in function and design to a more modern part of the building. All windows were exchanged by wooden windows, which all have good thermal values. The living area changed from a 4-flat house of $370 \mathrm{~m}^{2}$ for in average 5 person to a 7 -flat house for 12 residents. The heating requirement before renovation was 142 $\mathrm{kWh} / \mathrm{m}^{2} \mathrm{a}$ and could be reduced to a quarter of this. A thermal solar system now supports the former heating system of gas.

\begin{tabular}{|llll|}
\hline $\begin{array}{l}\text { House Maurer } \\
\text { Wolfurt, AT }\end{array}$ & Building Period: $1800-1849$ & Use: Residential (rural) & Contact: \\
Renovation: 2015 & Protection level: not listed & Energieinstitut Vorarlberg \\
\hline
\end{tabular}




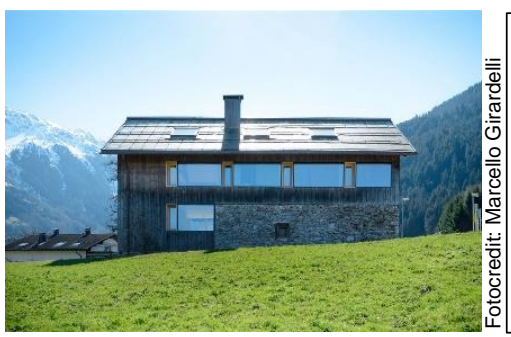

The associated farmhouse was demolished at the beginning of the 1970s. The aim was to convert the farm building into a high-quality residential building. In the process, the outer shell was supplemented by a few openings, which makes the new use of the building readable. The work on the original construction was carried out using old techniques wherever possible. Thus, the plugged connections are again in this form. The outer wooden facade was preserved entirely.

\begin{tabular}{|llll|}
\hline $\begin{array}{l}\text { House Breuer } \\
\text { Tschagguns, AT }\end{array}$ & Building Period: $1900-1944$ & $\begin{array}{l}\text { Use: Residential (rural) } \\
\text { Renovation: 2015 }\end{array}$ & Protection level: not listed \\
\hline
\end{tabular}

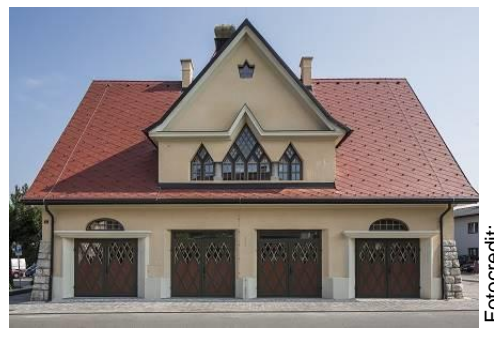

Restoration of a former fire station and transformation into a music school including a comprehensive thermal-energetic renovation. The renovation of the listed building was carried out within the framework of the demonstration programme "Mustersanierung".

\begin{tabular}{|llll|}
\hline $\begin{array}{l}\text { Music School Velden } \\
\text { Velden, AT }\end{array}$ & Building Period: 1900-1944 & $\begin{array}{l}\text { Use: Educational/Research } \\
\text { Renovation: 2015 }\end{array}$ & $\begin{array}{l}\text { Contact: } \\
\text { e7 energy innovation \& engineering }\end{array}$ \\
\hline
\end{tabular}

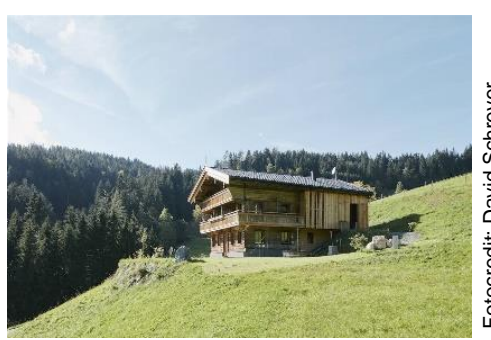

The 300-year-old "Hof Neuhäusl" is a prime example of the combination of old building stock and energy efficiency. While retaining its historical appearance, the building was refurbished completely in 2017. The preservation of the façade required the implementation of consistent interior insulation. Inside, the rooms were restructured in order to meet the highest, modern living standards. The revitalisation of the courtyard was awarded with the "Tiroler Sanierungspreis" in 2018.

\begin{tabular}{|llll|}
\hline $\begin{array}{l}\text { Hof Neuhäusl } \\
\text { Tirol, AT }\end{array}$ & Building Period: $1700-1800$ & Use: Residential (rural) & Contact: \\
Renovation: 2017 & Protection level: not listed & University of Innsbruck \\
\hline
\end{tabular}

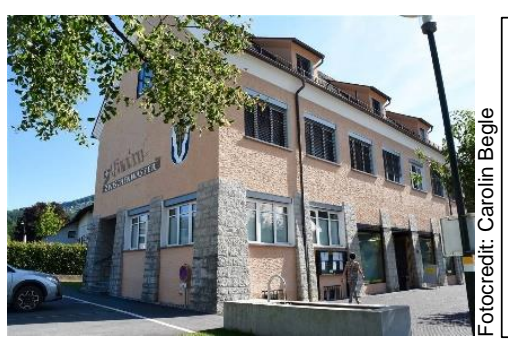

The comprehensive modernisation of the municipal office has now also been completed to a high standard. While the ground floor was on the raised ground floor before the conversion, large parts of this level have now been lowered to street level and glazed and thus converted into a barrier-free and transparent citizens' office. The renovation relies on night cooling and cross-ventilation. The reduction of the heating energy demand is achieved, among other things, by a wall system of interior insulation with clay plaster and at the same time makes it possible to leave the façade unchanged.

$\begin{array}{llll}\text { Community Hall } & \text { Building Period: 1900-1944 } & \text { Use: Community Hall } & \text { Contact: } \\ \text { Zwischenwasser, AT } & \text { Renovation: 2015 } & \text { Protection level: not listed } & \text { Energieinstitut Vorarlberg }\end{array}$

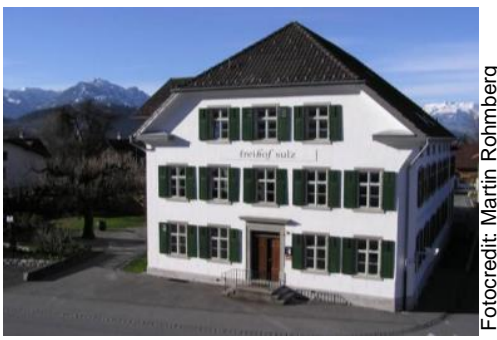

Renovation of ecological Freihof Sulz: Holistic redevelopment of the cultural heritage "Freihof Sulz", which is worthy of preservation, into a lively meeting place. A demonstration object for local culture and quality of life, old building techniques, as well as energy-saving and ecological renovation.

\begin{tabular}{|llll|}
\hline $\begin{array}{l}\text { Freihof Sulz } \\
\text { Sulz, AT }\end{array}$ & Building Period: $1700-1800$ & Use: Restaurant/Stores/Practices & Contact: \\
Renovation: 2006 & Protection level: listed & Energieinstitut Vorarlberg \\
\hline
\end{tabular}




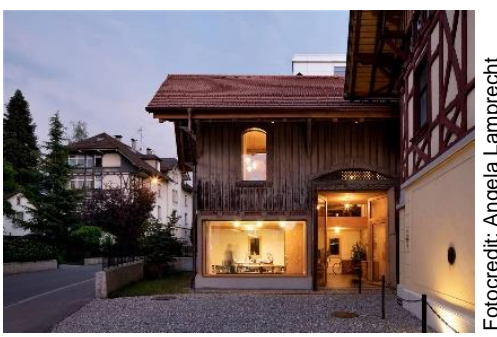

The potential that lies dormant in vacant properties and which must be exploited is shown by the "Öeconomicgebäude" in Dornbirn, which has been brought back to life. Contemporary living and working are being introduced into the sensitively adapted urban barn, which is a listed building. For Julia Kick, who acted in this project in the dual role of architect and builder, the "Oeconomiegebäude" was a stroke of luck, because in her work she has been dealing intensively with the topic of vacancy for several years.

\begin{tabular}{|llll|}
\hline $\begin{array}{l}\text { Oeconomy-Building } \\
\text { Dornbirn, AT }\end{array}$ & $\begin{array}{l}\text { Building Period: } 1850-1899 \\
\text { Renovation: } 2016\end{array}$ & $\begin{array}{l}\text { Use: Residential with atelier } \\
\text { Protection level: listed }\end{array}$ & $\begin{array}{l}\text { Contact: } \\
\text { Energieinstitut Vorarlberg }\end{array}$ \\
\hline
\end{tabular}

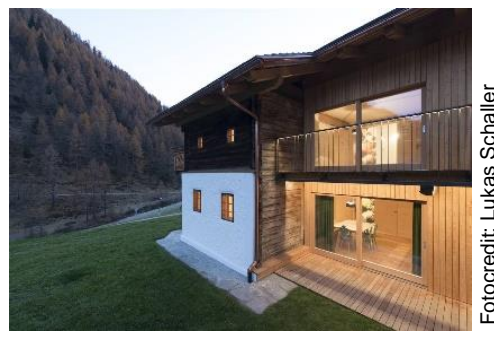

The Giatla house, a 300-year-old farmhouse typical of the region, had been neglected and was in a very devastated condition. There were some indications that the house was sliding down the slope. A new use as a holiday apartment and a careful intervention has brought this building back to life.

\begin{tabular}{|llll|}
\hline $\begin{array}{l}\text { Giatla House } \\
\text { Innervillgraten, AT }\end{array}$ & Building Period: $1600-1700$ & $\begin{array}{l}\text { Use: Hotel/Restaurant } \\
\text { Renovation: 2015 }\end{array}$ & $\begin{array}{l}\text { Contact: } \\
\text { University of Innsbruck }\end{array}$ \\
\hline
\end{tabular}

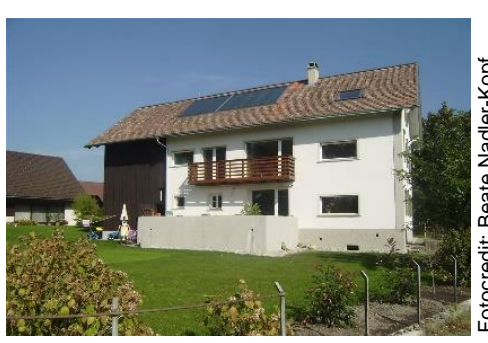

The Irgang family has been dealing with the idea of demolishing the old Rhine valley house and building a new one for a long time. But this idea finally gave way to the advantages of the renovation. Decisive factors were the floor plans which offered many possibilities, the family-related history, the charm of the old Rhine Valley House and last but not least the comparison of costs and usable space.

\begin{tabular}{|llll|}
\hline $\begin{array}{l}\text { Rhine Valley House Irgang } \\
\text { Rankweil, AT }\end{array}$ & Building Period: $1850-1899$ & Use: Residential (rural) & Contact: \\
Renovation: 2008 & Protection level: not listed & Energieinstitut Vorarlberg \\
\hline
\end{tabular}

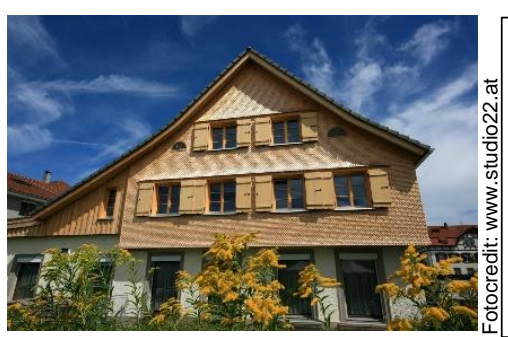

"Cozy, comfortable, plesant - our home! The atmosphere of old and new, the special character and the history that come with an old house, make it something special. Based on experience we can say: dare to preserve old building fabric. You won't regret it!" says the owner. Built in 1878, the house has always been owned by the same family. In 2011 the Rhine-valley house was renovated by the great-great-great-grandson of the builder with a lot of passion and love for detail.

\begin{tabular}{|llll|}
\hline $\begin{array}{l}\text { Baur Residence } \\
\text { Lustenau, AT }\end{array}$ & Building Period: $1850-1899$ & $\begin{array}{l}\text { Use: Residential (rural) } \\
\text { Protection level: not listed }\end{array}$ & Contact: \\
Energieinstitut Vorarlberg
\end{tabular}

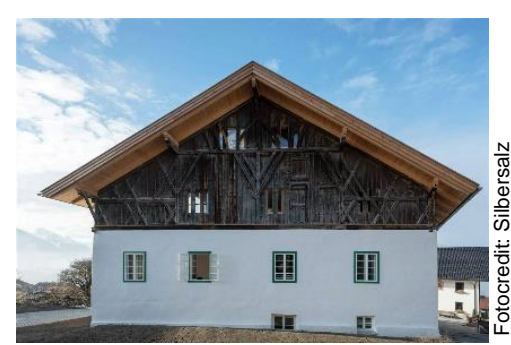

The revitalisation of the village centre and its versatile use, with an area of about $650 \mathrm{~m}^{2}$, has been realised by the refurbishment of a former farm, the Kasperhof in Patsch, which had been vacant for more than 20 years.

\begin{tabular}{|llll|}
\hline $\begin{array}{l}\text { Kasperhof } \\
\text { Patsch, AT }\end{array}$ & Building Period: $1600-1700$ & Use: Residential (rural) & Contact: \\
Renovation: 2019 & Protection level: not listed & University of Innsbruck \\
\hline
\end{tabular}




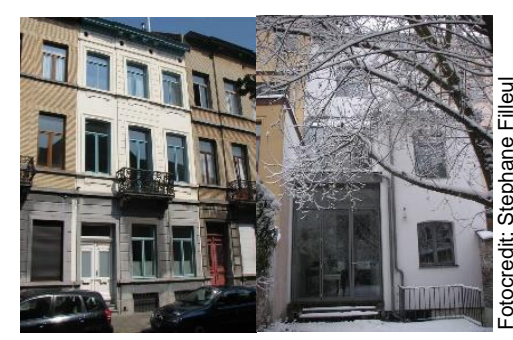

The "maison Rubens" is a typical middle-class row house from late 19th century, in neoclassical style. It reflects the Belgium bourgeoisie life at this period. Since 1888 (date of construction) no major renovation had been done. The building was almost in its original condition, but in very bad conservation state. The renovation followed two main goals: i) very good insulation (using bio-based materials) and mechanical ventilation of the whole house while conserving the valuable heritage details. ii) energy consumption below $60 \mathrm{kWh} / \mathrm{m}^{2} \mathrm{y}$.

\begin{tabular}{|llll|}
\hline $\begin{array}{l}\text { Maison Rubens } \\
\text { Schaerbeek, BE }\end{array}$ & Building Period: $1850-1899$ & Use: Residential (urban) & Contact: \\
\hline
\end{tabular}

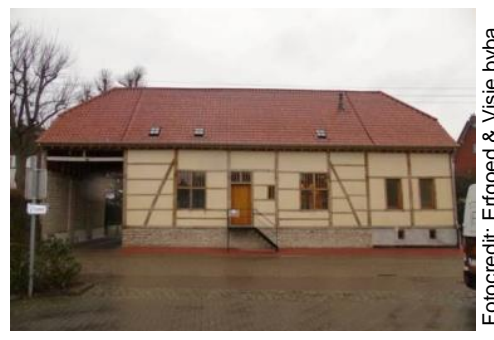

Half-timber framed building with late 17th century core, expanded on both sides in the 19th century. The building was out of use and in a very bad state at the moment of renovation. The purpose of the total building renovation (including improvement of energy performance and comfort) was residential reuse. The building renovation comprehended renovation of the roof, the external walls and windows, floor insulation as well as some other interventions such as introduction of a new condensing gas wall boiler, underfloor heating for the ground floor and radiators for the first floor.

\begin{tabular}{|llll|}
\hline $\begin{array}{l}\text { Half-timberframed house } \\
\text { Alken, BE }\end{array}$ & Building Period: $1600-1700$ & $\begin{array}{l}\text { Use: Residential (rural) } \\
\text { Protection level: listed }\end{array}$ & $\begin{array}{l}\text { Contact: } \\
\text { Flanders Heritage Agency }\end{array}$ \\
\hline
\end{tabular}

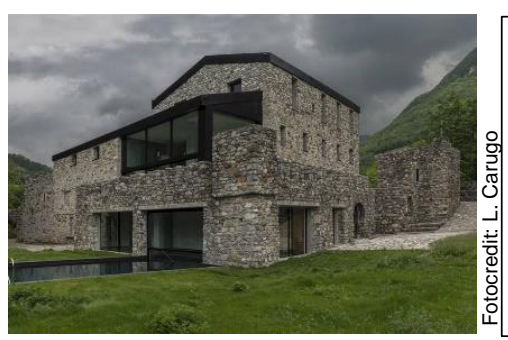

Single-family house, restoration and new building extension. Private residence building (historic not-listed building in Ticino) with NZEB target using also solar renewables energy in an integrated roof solution. The castle of Doragno was born from the transformation of an ancient medieval castle. The original stone walls are highlighted by the large windows that complete the volume of the existing building. An integrated photovoltaic system and solar collectors are installed on the roof.

\begin{tabular}{|llll|}
\hline $\begin{array}{l}\text { Doragno Castle } \\
\text { Rovio, } \text { CH }\end{array}$ & Building Period: before 1600 & Use: Residential (rural) & Contact: \\
Penovation: 2017 & Protection level: not listed & SUPSI \\
\hline
\end{tabular}

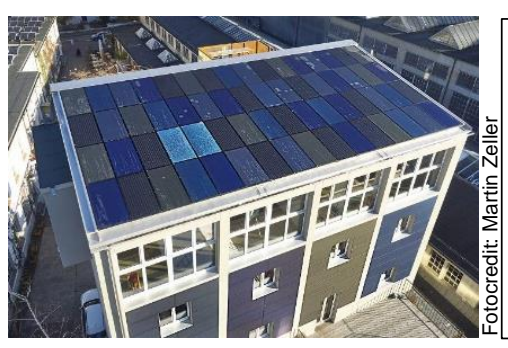

This best practice building in "Grundelinger Feld" investigates new approaches for BIPV integration as cladding innovative materials and new energy storage strategies. As "Gundeldinger Feld" ensemble is under heritage protection, the remodelled building was required to match the style and colour scheme of the site. The project is part of the "2000 - Watt society - pilot region Basel" and was rewarded in the "renovation" category with the 2015 Swiss Solar Prize.

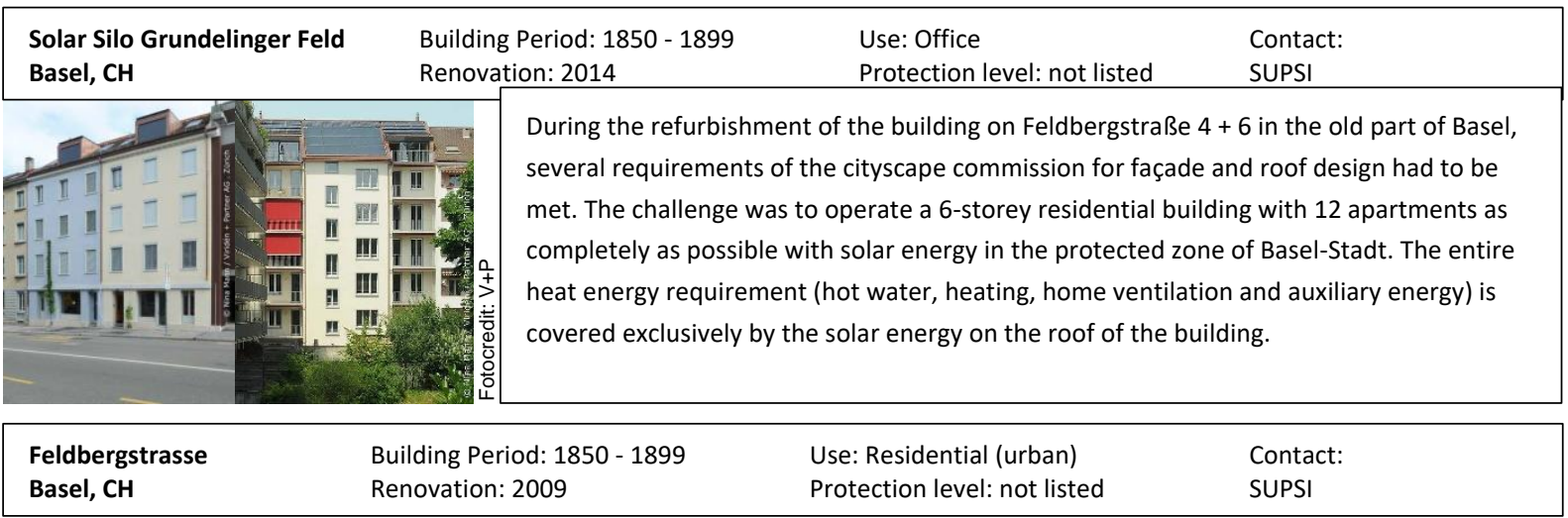




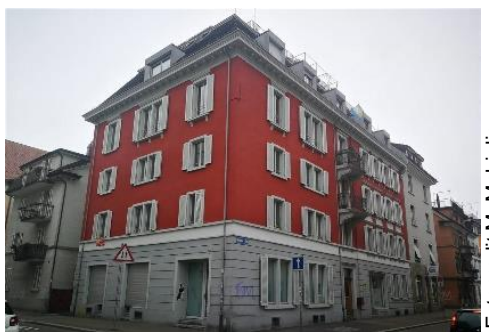

The multi-family house in Zurich needed a complete modernization. Thanks to the optimal use of building regulations and the vision of the architects, the new roof could be raised on the courtyard side so far that a new storey and thus more living space was created. Despite preservation requirements, the building could be well insulated today reaches the Minergie new construction standard. For the placement of solar collectors, the roof was not optimally aligned and too small. Rentable terrace and energy-collecting panels must therefore share the space on the roof.

\begin{tabular}{|llll|}
\hline Magnusstrasse & Building Period: $1850-1899$ & Use: Residential (urban) & Contact: \\
Zürich, $\mathbf{C H}$ & Renovation: 2007 & Protection level: listed & SUPSI \\
\hline
\end{tabular}

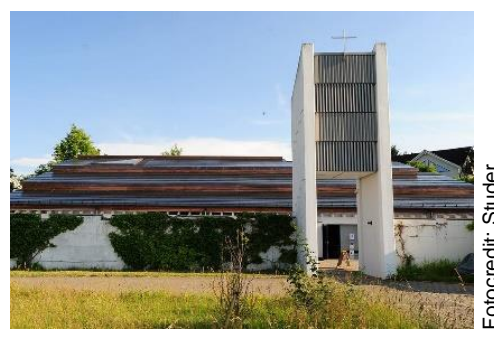

The Roman Catholic St. Franziskus Ebmatingen Church is emission-free after the energetic renovation in 2018/19. Thanks to significantly improved roof insulation, the solarpowered geothermal heat pump, the use of solar heat with $161 \mathrm{~m} 2$ of photovoltaic thermal modules (PVT) and the LED lighting the church is a plus-Energy-Building with an energy supply of $221 \%$. For this concept in combination with the architecturally outstanding implementation, the project has been awarded in 2019 with the Swiss and European Solar Prize.

\begin{tabular}{|llll|}
\hline $\begin{array}{l}\text { St. Franziskus Church } \\
\text { Ebmatingen, } \mathbf{C H}\end{array}$ & Building Period: 1980 & Use: Religious & Contact: \\
Renovation: 2018 & Protection level: not listed & SUPSI \\
\hline
\end{tabular}

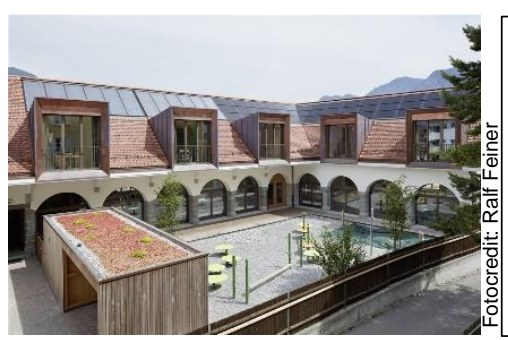

The building complex with a double kindergarten and two penthouses built in 1914 consumed 184,300 kWh/a before the renovation. Thanks to the good thermal insulation and the use of direct solar generation, the total energy requirement was reduced by $84 \%$ to $29,400 \mathrm{kWh} / \mathrm{a} .79 \mathrm{~m}^{2}$ of the latest generation of solar panels bring a lot of light and heat into the building. Inside there is enough mass to absorb the heat and store it above ground.

\begin{tabular}{|llll|}
\hline $\begin{array}{l}\text { Kindergarten and apartments } \\
\text { Chur, } \mathbf{C H}\end{array}$ & Building Period: $1900-1944$ & $\begin{array}{l}\text { Use: Educational/Research } \\
\text { Protection level: not listed }\end{array}$ & $\begin{array}{l}\text { Contact: } \\
\text { SUPSI }\end{array}$ \\
\hline
\end{tabular}

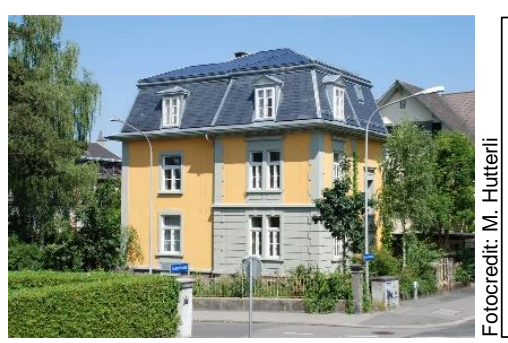

The neo-baroque style house of the Hutterli Röthlisberger family has been extensively renovated and thermally refurbished between 2011 and 2015. The intervention concerns the thermal improvement of the envelope, intervening on walls, roof and windows. It also concerns the installation of a photovoltaic system and a solar thermal system, as well as the replacement of the gas heating system with a heat pump, geothermal probes and a stove. The intervention also involved the installation of a comfort ventilation system with heat recovery. This renovation deserved the Swiss Solar Prize 2014.

\begin{tabular}{|lll}
$\begin{array}{l}\text { Single family house } \\
\text { Bern, } \mathrm{CH}\end{array}$ & $\begin{array}{l}\text { Building Period: } 1850 \text { - } 1899 \\
\text { Protection level: listed }\end{array}$ \\
Renovation: 2015 & SUPSI
\end{tabular}

\begin{tabular}{|llll|}
\hline $\begin{array}{l}\text { Single family house } \\
\text { Gstaad, CH }\end{array}$ & Building Period: $1700-1800$ & Use: Residential (rural) & Contact: \\
Renovation: 2018 & Protection level: listed & SUPSI \\
\hline
\end{tabular}




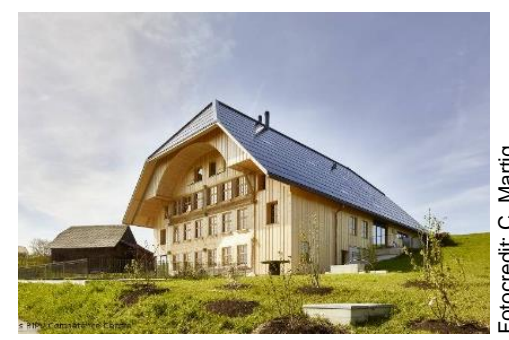

This restoration is connected with the aim of preserving the overall appearance of the building, repairing the roof, facades and surroundings and carefully restoring the prestigious south facade. From a technical point of view, the building is solidly stabilised and energetically brought up to the latest standards. The project was developed with the involvement of the cantonal monument preservation authorities. Several meetings and inspections took place, on the basis of which the project was further developed in accordance with the requirements of the preservation of historical monuments.

\begin{tabular}{|llll|}
\hline $\begin{array}{l}\text { Glaserhaus } \\
\text { Affoltern, CH }\end{array}$ & Building Period: $1700-1800$ & Use: Residential (rural) & Contact: \\
Renovation: 2015 & Protection level: listed & SUPSI \\
\hline
\end{tabular}

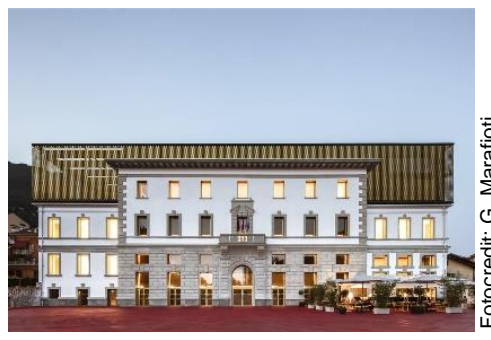

The Palazzo del Cinema Locarno project is guided by principles of economy, trying to capitalise in the existing structure and the public affection for the Palazzo Scolastico, to provide an architectural identity for the new cinema complex in Piazza Remo Rossi. At a time when energy resources are dwindling and climate change has become a crucial problem for our cities, it would have been irresponsible to simply discard the existing building in order to build an entirely new one, with the corresponding expenditure of vital resources. Urban Recycling is a more adequate strategy for this intervention.

\begin{tabular}{|llll|}
\hline $\begin{array}{l}\text { PalaCinema } \\
\text { Locarno, CH }\end{array}$ & Building Period: $1850-1899$ & $\begin{array}{l}\text { Use: Multicultural platform for cinematic arts } \\
\text { Protection level: not listed }\end{array}$ & $\begin{array}{l}\text { Contact: } \\
\text { SUPSI }\end{array}$ \\
\hline
\end{tabular}

\begin{tabular}{|l}
\begin{tabular}{llll|}
\hline $\begin{array}{l}\text { Casa Rossa Chemnitz } \\
\text { Chemnitz, DE }\end{array}$ & Building Period: $1900-1944$ & Use: Residential (urban) & Contact \\
Renovation: 2018 & Protection level: not listed & Fraunhofer ISE \\
\hline
\end{tabular}
\end{tabular}

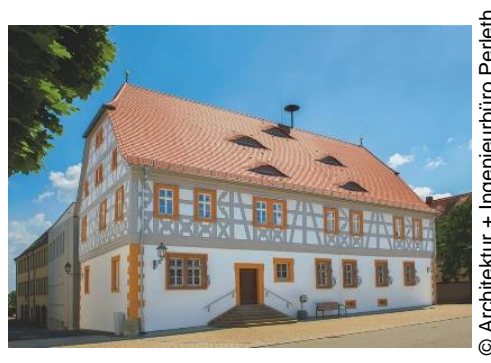

The listed town hall in Bergrheinfeld was built in 1660 and has been converted and renovated again and again over the past centuries. The most recent restoration of the cultural heritage included, in addition to the existing old building, the construction of a modern, barrier-free extension building with a textile facade and together they form the new and modern center of the community.

\begin{tabular}{|llll|}
\hline Town Hall & Building Period: $1600-1700$ & Use: Town Hall & Contact: \\
Bergrheinfeld, DE & Renovation: 2018 & Protection level: listed & EURAC Research \\
\hline
\end{tabular}

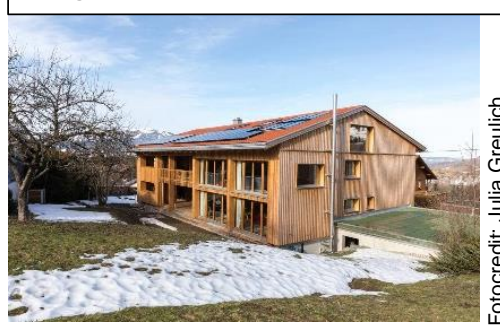

Authentic, energy-efficient and ecological: the Straub family had specifically looked for an old farm; together with the architect, they succeeded in creating a cosy home. For the most part, the traditional use was retained, in some places reinterpreted for today's requirements. The historic building fabric from the 18th century was in good condition and was preserved as well as staged with modern materials. This also succeeds under the premise of sustainability, through insulation with cellulose fibres as well as a pellet boiler and solar panels for heating and hot water.

$\begin{array}{llll}\begin{array}{l}\text { Farmhouse Straub } \\ \text { Sonthofen, DE }\end{array} & \text { Building Period: } 1700-1800 & \text { Use: Residential (rural) } & \text { Contact: } \\ & \text { Renovation: 2018 } & \text { Protection level: not listed } & \text { EURAC Research }\end{array}$




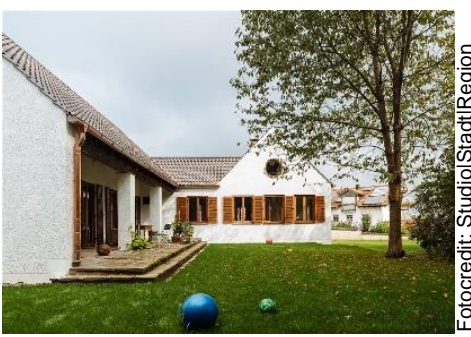

The single-family house was built in 1936 by Sep Rüf for the writer Josef Martin Bauer. It consists of two single-story structures with gable roofs, which are arranged in a Z-shape to each other, thus forming an entrance courtyard and a terrace courtyard. In order to be able to use the building as a residential house in 2013 , it had to be extensively renovated. For this purpose, a differentiated renovation concept was developed, which provided for specific measures and procedures for the building envelope and the interior respectively.

\begin{tabular}{|llll|}
\hline $\begin{array}{l}\text { Frühwerk Sep Ruf } \\
\text { Dorfen, DE }\end{array}$ & Building Period: $1900-1944$ & Use: Residential (rural) & Contact: \\
Renovation: 2014 & Protection level: listed & EURAC Research \\
\hline
\end{tabular}

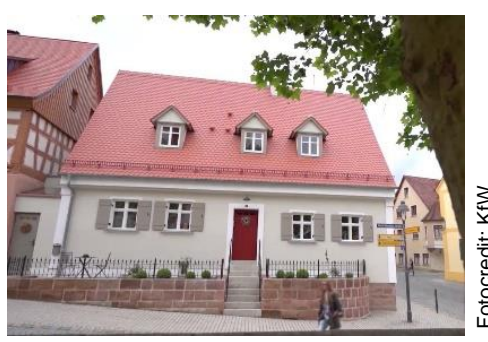

The "Ackerbürgerhäuschen" is located in middle Franconia and was built around 1400. The building is listed for preservation and is situated in the old town of Hilpoltstein, which is listed as well. During the renovation of the Ackerbürgerhäuschen in 2016, Monika and Thomas Fritsch set themselves the goal of preserving not only the possibly 1,000-year-old cellar vault, but also the outer wall made of sandstone and the oak beams of the framework and roof truss. The house got the KfW Award "Bauen und Wohnen 2016" because of the excellent energetic renovation.

\begin{tabular}{|c|c|c|c|}
\hline Ackerbürgerhäuschen & Building Period: before 1600 & Use: Residential (rural) & Contact: \\
\hline Hilpoltstein, DE & Renovation: 2015 & Protection level: listed & EURAC Research \\
\hline
\end{tabular}

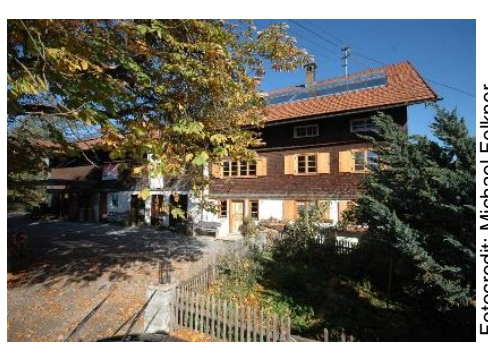

Step by step, always following the principle of the best possible solution, architect Michael Felkner is renovating the Ritterhof: the farmhouse from the late 17th century now houses his office and the flat for the family, but also a health food shop and a granny flat converted for age-appropriate living. The remaining heating needs are covered by wood and solar energy.

\begin{tabular}{|llll|}
\hline $\begin{array}{l}\text { Ritterhof } \\
\text { Oberdorf, DE }\end{array}$ & Building Period: $1850-1899$ & Use: Residential (rural) & Contact: \\
Renovation: 2016 & Protection level: not listed & EURAC Research \\
\hline
\end{tabular}

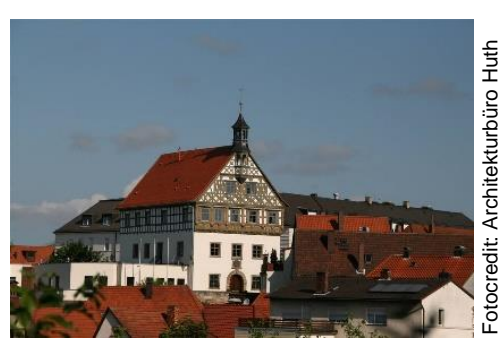

On the site of the former "Altenburg ob Kunstadt", the Burgkunstädter town hall has formed the town's skyline for over 300 years. In 1689/90, the master of baroque halftimbered construction, Jörg Hoffmann constructed the impressive decorative halftimbered structure on the early medieval masonry base. After an inadequate restoration in the 1970s, considerable damage was found on the entire building in 2000. The aim of the overall renovation of the old building was to preserve and emphasise the historical value of this important monument and to equip it for the demands of the present day.

\begin{tabular}{|llll|}
\hline $\begin{array}{l}\text { Town Hall } \\
\text { Burgkunstadt, DE }\end{array}$ & $\begin{array}{l}\text { Building Period: before } 1600 \\
\text { Renovation: } 2009\end{array}$ & $\begin{array}{l}\text { Use: Town Hall } \\
\text { Protection level: listed }\end{array}$ \\
EURAC Research
\end{tabular}

\begin{tabular}{|llll|}
\hline $\begin{array}{l}\text { Osramhuset } \\
\text { Copenhagen, DK }\end{array}$ & $\begin{array}{l}\text { Building Period: 1945-1959 } \\
\text { Renovation: 2009 }\end{array}$ & $\begin{array}{l}\text { Use: Culture and community centre } \\
\text { Protection level: listed }\end{array}$ & $\begin{array}{l}\text { Contact: } \\
\text { Danish Building Research Institute }\end{array}$ \\
\hline
\end{tabular}




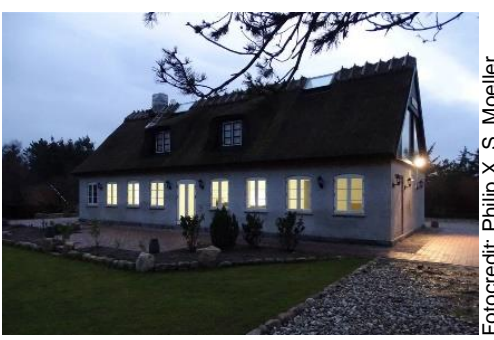

Klitgaarden is a single-family house from 1875 in two stories with a total of $221 \mathrm{~m}^{2}$. The building is erected with solid masonry walls and a foundation of granite boulders on top of a stone foundation. The renovation project of the building aimed for a minimum heating requirement and a preservation of original outdoor facade details. It was finished in 2016. The renovation was motivated by a very high-energy demand for heating and in general the fact that the house had not been used in twenty years.

\begin{tabular}{|llll|}
\hline $\begin{array}{l}\text { Klitgaarden } \\
\text { Hundested, DK }\end{array}$ & Building Period: $1850-1989$ & Use: Residential (rural) & Contact: \\
Renovation: 2016 & Protection level: not listed & Danish Building Research Institute \\
\hline
\end{tabular}

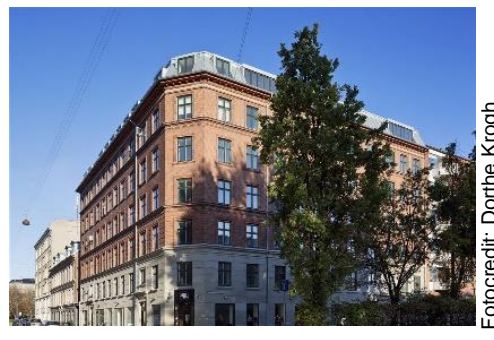

This typical residential building from the end of the 19th century, was outdated and in need for renovation. The indoor climate was poor and the energy consumption quite high. After a renovation that included new windows, thermal insulation on walls and ceiling, improved air-tightness, mechanical ventilation with heat recovery, and photovoltaic, the building achieved a $63 \%$ reduction in energy demand.

\begin{tabular}{|llll|}
\hline $\begin{array}{l}\text { RYESGADE 30 } \\
\text { Copenhagen, DK }\end{array}$ & Building Period: 1850 - 1899 & $\begin{array}{l}\text { Use: Residential (urban) } \\
\text { Protection level: listed }\end{array}$ & $\begin{array}{l}\text { Contact: } \\
\text { Danish Building Research Institute }\end{array}$ \\
\hline
\end{tabular}

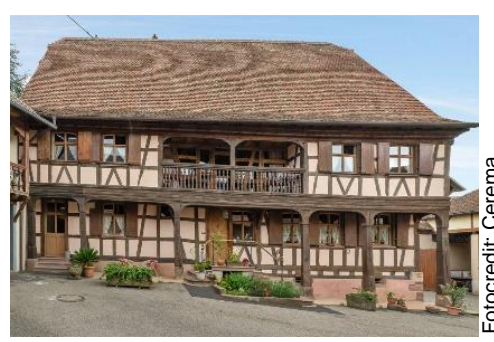

This timber-framed house is located in Schnersheim in the Alsace region (north-eastern France), near the border with Germany. It belongs to the same family since the 17th century and it has recently been retrofitted and restored. The project reaches a balance between low energy consumption and heritage preservation. The house is one of the case studies of the CREBA (French knowledge center for responsible retrofit of heritage building) website.

\begin{tabular}{|llll|}
\hline $\begin{array}{l}\text { Timber-framed house Alsace } \\
\text { Schnersheim, FR }\end{array}$ & Building Period: $1700-1800$ & Use: Residential (rural) & Contact: \\
Renovation: 2015 & Protection level: not listed & Cerema \\
\hline
\end{tabular}

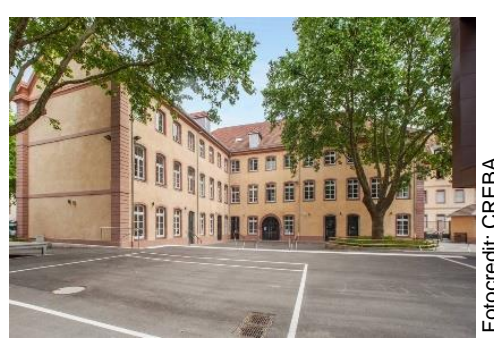

This hard-stone elementary school is located in Mulhouse in the Alsace region (northeastern France), near the border with Germany. It is a listed building: it first was a spinning factory at a time when Mulhouse was well-known for its textile industry and became a school after the annexation of Alsace and Moselle by Germany in 1870. The project reaches a balance between low energy consumption and heritage preservation, despite a constraint budget. The school is one of the case studies of the CREBA (French knowledge center for responsible retrofit of heritage building) website.

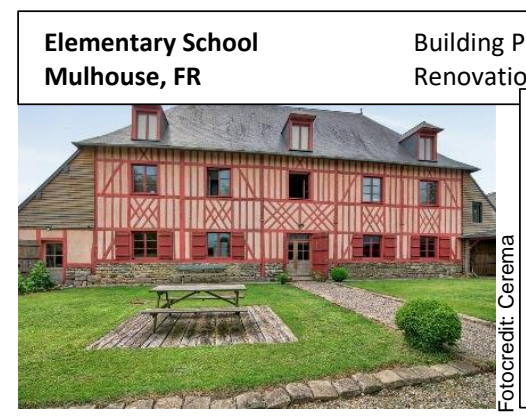

Use: Educational/Research
Protection level: listed

Contact: Cerema

This timber-framed building is located in Saint-Samson-la-Poterie in the Oise region (northern France), over Paris, and especially in the natural region called "Pays de Bray". It was the barn of a landlord housing of the 17th century and it has recently been retrofitted and restored by the owner himself. A careful work on the materials and on the execution was made. The barn is one of the case studies of the CREBA (French knowledge center for responsible retrofit of heritage building) website.

\begin{tabular}{|llll|}
\hline Timber-framed barn North France & Building Period: $1700-1800$ & Use: Residential (rural) & Contact: \\
Saint-Samson-la-Poterie, FR & Renovation: 2016 & Protection level: not listed & Cerema \\
\hline
\end{tabular}




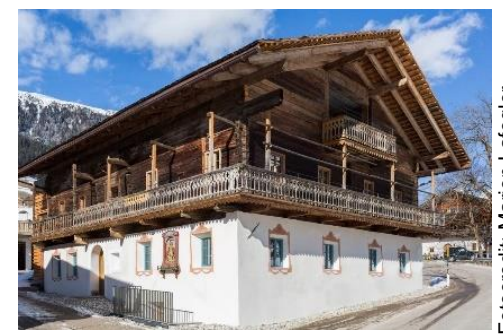

The Rainhof is one of the most precious rural buildings of the Gsiesertal valley, at $1.500 \mathrm{~m}$ above sea level. Built with solid stone masonry walls and the vernacular "Blockbau" (solid wood) construction, the building presents many traditional features, such as windows with deep reveals, decorated painted frames around the windows, and vaulted ceilings. The project was awarded the 1st prize at the Bauern(h)auszeichnung - ITAS-Preis 2016 for the best renovation of a farmhouse in Südtirol.

\begin{tabular}{|llll|}
\hline Rainhof & Building Period: before 1600 & Use: Residential (rural) & Contact: \\
Gsies, IT & Renovation: 2016 & Protection level: listed & EURAC Research \\
\hline
\end{tabular}

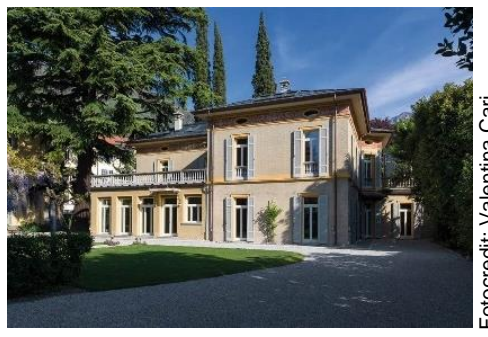

Villa Castelli is a listed building from the 19th century located at the riverside of Lake Como (Italy). The owners set the ambitious goal of renovating the Villa, which had belonged to the family for about 140 years, to the lowest possible energy demand while maintaining the original use of the rooms and the external appearance. The renovation achieved a $90 \%$ energy demand reduction and a significant increase in comfort, demonstrating that also a listed building can become nZEB.

\begin{tabular}{|llll|}
\hline $\begin{array}{l}\text { Villa Castelli } \\
\text { Bellano, IT }\end{array}$ & Building Period: $1850-1899$ & $\begin{array}{l}\text { Use: Residential (rural) } \\
\text { Protection level: listed }\end{array}$ & $\begin{array}{l}\text { Contact: } \\
\text { EURAC Research }\end{array}$ \\
\hline
\end{tabular}

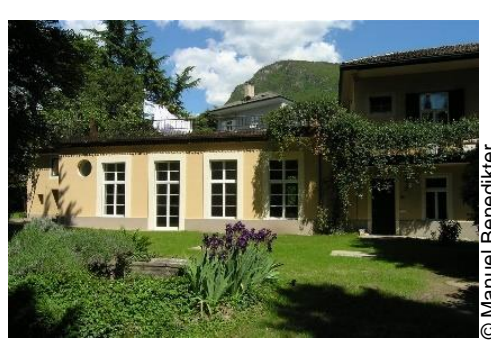

Retrieval of lost Orangerie character and energy retrofit - these were the targets which the owner aimed for. He showed that factor 10 reduction in energy demand is possible also in a listed building.

\begin{tabular}{|llll|}
\hline $\begin{array}{l}\text { Ansitz Kofler } \\
\text { Bozen, IT }\end{array}$ & Building Period: $1700-1800$ & Use: Residential (urban) & Contact \\
Renovation: 2008 & Protection level: listed & EURAC Research \\
\hline
\end{tabular}

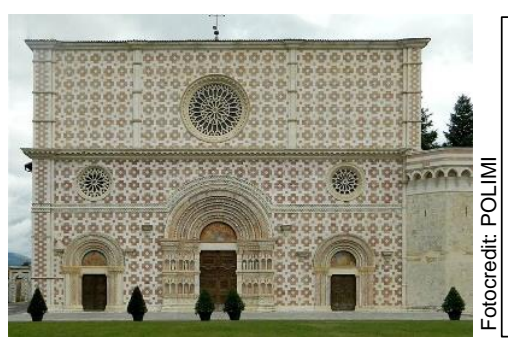

Basilica di Santa Maria di Collemaggio L'Aquilia, IT

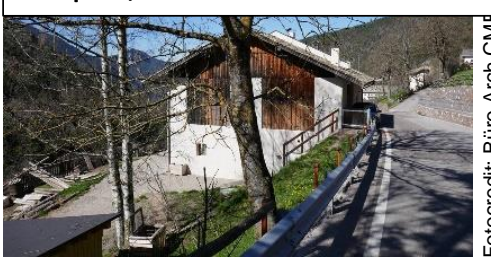

House Pernter

Truden, IT
The Basilica di S. Maria di Collemaggio, L'Aquila, is a masterpiece of Abruzzese Romanesque and Gothic architecture and a very important religious site for the original Papal Jubilee devised by Pope Celestine V, who is buried there. The main challenge was to design a heating system comprehensive of its connections and pipes without interfering in the original appearance of the church, able at the same time to preserve the cultural heritage of the Basilica. The restoration of the Basilica was financially supported by ENI after demolition by an earthquake.

The renovation of the house Pernter in Truden is based on the character of the village. The building from 1923 was formerly used entirely for agricultural purposes. The typical Tyrolean style of the construction is expressed in the compact design with residential house, stable and barn under one roof. The aim of the renovation was to optimise the energy balance without altering the existing building envelope. Emphasis was placed on the use of regional materials and the preservation of old building elements to preserve the atmosphere of the farmhouse. 


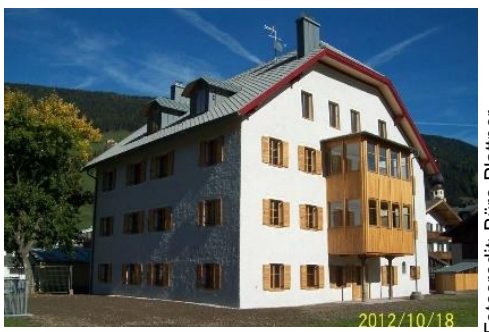

The Kohlerhaus, a building dating back to the 14th century, was renovated to accommodate 10 apartments with the high standards of comfort and efficiency. The historical research carried out by the architect revealed the rich past of the building, that served as priest house, hotel or guest house. The retrofit of the building included external insulation the wall with reed mats, new replica windows and new building services (radiating heating and mechanical ventilation).

\begin{tabular}{|llll|}
\hline $\begin{array}{l}\text { Kohlerhaus } \\
\text { Innichen, IT }\end{array}$ & Building Period: before 1600 & Use: Residential (urban) & Contact: \\
& Renovation: 2011 & Protection level: not listed & EURAC Research \\
\hline
\end{tabular}

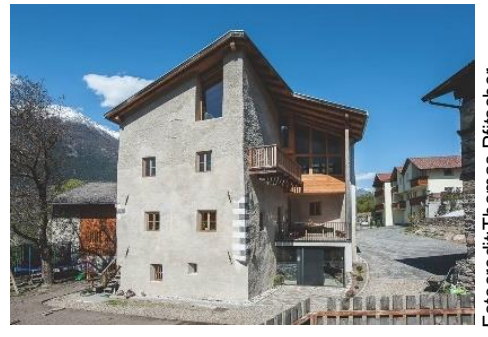

In the village of Tarsch, in the middle of an inner alpine location, the "Ruckenzaunerhof" presents itself as a typical, Vinschgau stone building. The oldest parts of the farm can be dated back to the 15th century. Combined with new components, which have been sensitively integrated into the historical building, they now form a harmonious unit. The project was awarded the ITAS prize in $\mathbf{2 0 1 5}$ for the best renovation of a farmhouse in South Tyrol.

\begin{tabular}{|llll|}
\hline $\begin{array}{l}\text { Ruckenzaunerhof } \\
\text { Tarsch, IT }\end{array}$ & Building Period: before 1600 & Use: Residential (rural) & Contact: \\
Renovation: 2015 & Protection level: listed & EURAC Research \\
\hline
\end{tabular}

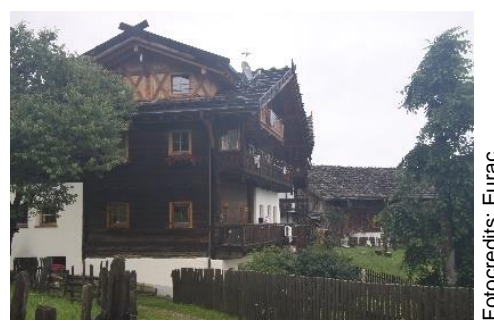

The farmhouse of the Aussergrubhof is situated in an idyllic landscape on a sunny slope above St. Nikolaus in Ulten. Although the farm house is not listed, the Thöni family was committed to maintain the historical character of their home. The Aussergrubhof was awarded with the ITAS prize in 2014 as a remarkable example for renovation.

\begin{tabular}{|llll|}
\hline $\begin{array}{l}\text { Aussergrubhof } \\
\text { Ulten, IT }\end{array}$ & Building Period: $1600-1700$ & Use: Residential (rural) & Contact: \\
Renovation: 2014 & Protection level: not listed & EURAC Research \\
\hline
\end{tabular}

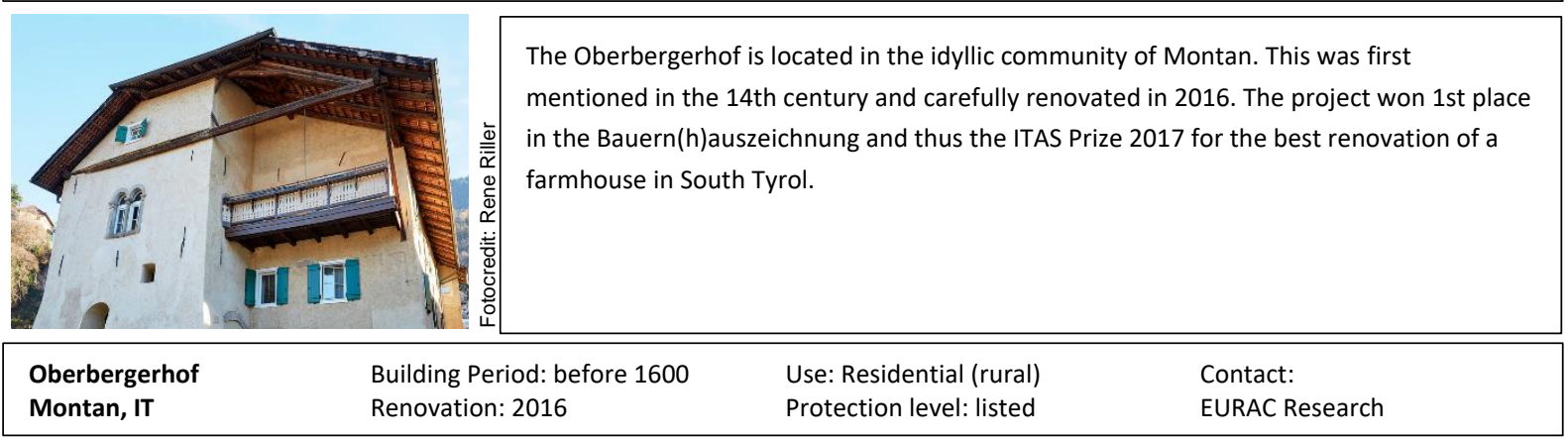

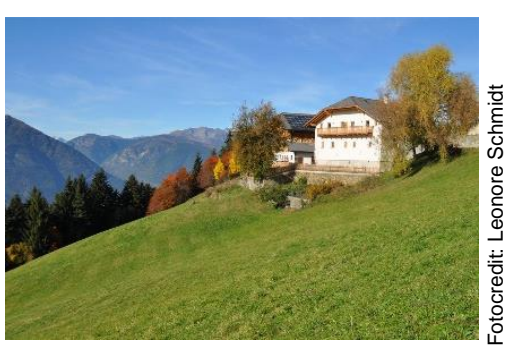

The mountain farm is located above St. Andrä, with a wide view over the Eisack valley. There are only meadows and woods all around, no traffic noise disturbs the peace and quiet. The old farmhouse has been renovated with loving attention to detail, while at the same time paying attention to a biological construction method and the use of regional materials.

\begin{tabular}{|llll|}
\hline $\begin{array}{l}\text { Platzbonhof } \\
\text { Brixen, IT }\end{array}$ & Building Period: before 1600 & Use: Residential (rural) & Contact: \\
Renovation: 2016 & Protection level: not listed & EURAC Research \\
\hline
\end{tabular}




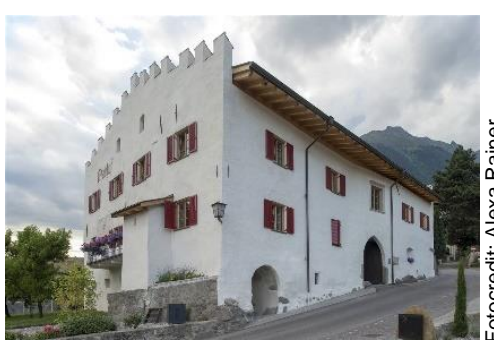

Mairhof, as listed farmer house, is situated on a hillside in the immediate vicinity of the centre of Partschins in Vinschgau. The rural house is characterised by its crenellated gables on the narrow sides, net vaulted corridors on ground and upper floor, as well as original "Stuben" and wooden ceilings with over 800 -year-old beams. The farm was awarded with the ITAS prize in 2018 for its first-class retrofit measures.

\begin{tabular}{|llll|}
\hline $\begin{array}{l}\text { Mairhof } \\
\text { Partschins, IT }\end{array}$ & Building Period: before 1600 & Use: Residential (rural) & Contact: \\
& Renovation: 2018 & Protection level: listed & EURAC Research \\
\hline
\end{tabular}

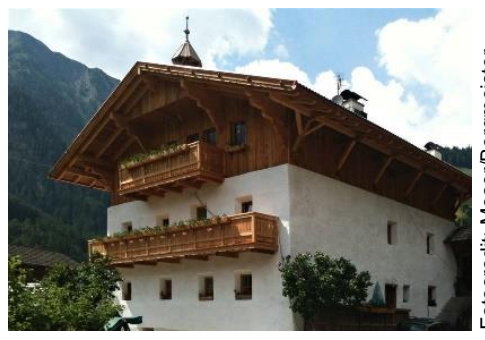

The "Obergasserhof" is located above the village of Pfunders along the country road, about $200 \mathrm{~m}$ into the valley. The farm is engaged in dairy and cattle farming and has recently started renting a holiday apartment as a sideline. The primary goal of the renovation measures in the residential building was to secure the architectural heritage.

\begin{tabular}{|llll|}
\hline $\begin{array}{l}\text { Obergasserhof } \\
\text { Vintl, IT }\end{array}$ & Building Period: before 1600 & Use: Residential (rural) & $\begin{array}{l}\text { Contact: } \\
\text { Renovation: 2013 }\end{array}$ \\
\hline
\end{tabular}

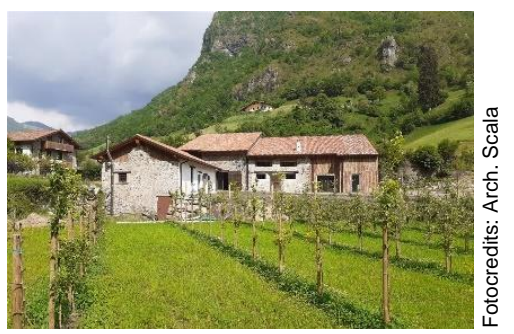

'Rebecco Farm' project is part of the "Resilient Valleys" program aimed to give new life to the area of the high valleys Trompia and Sabbia by enhancing their cultural, historical and environmental potential. From an architectural point of view, the complex consists in two stone-wall buildings, in a state of abandonment and partially collapsed, with a covered area of about $300 \mathrm{~m}^{2}$. Given its strategic position, the rural complex of 'Rebecco Farm' has been retrofitted to house a bed and breakfast and an educational service, for training and aggregation activities linked to the territory agricultural knowledge.

\begin{tabular}{|llll|}
\hline Rebecco Farm & Building Period: before 1600 & Use: B\&B - Farm & Contact: \\
Brescia, IT & Renovation: 1980 & Protection level: not listed & Politecnico Milano \\
\hline
\end{tabular}

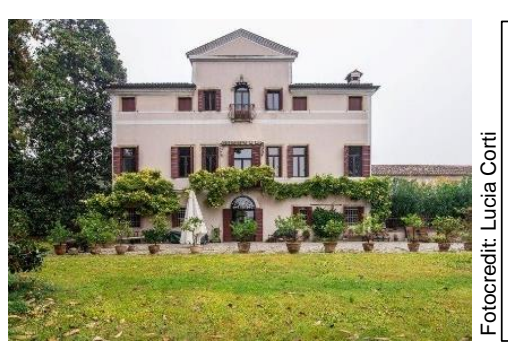

The restoration of Villa Capodivacca in the province of Padua is among those we can define "low impact". The combination of new functions and adequate technical solutions has allowed the historical characteristics and the original atmosphere of the building to emerge that have been handed down over the centuries. From the intersection of the differentiated use of the three floors of the villa, the problems encountered and the constraints imposed by the Superintendence, an articulated project of restoration, static restoration and plant and energy renovation was born.

\begin{tabular}{|llll|}
\hline $\begin{array}{l}\text { Villa Capodivacca } \\
\text { Saccolongo, IT }\end{array}$ & Building Period: before 1600 & Use: Residential (rural) & Contact: \\
Renovation: 2017 & Protection level: listed & University of Genoa \\
\hline
\end{tabular}

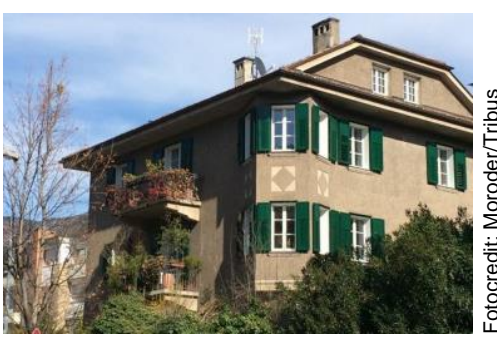

The historic residential building is located in the city center of Bolzano. It was built in 1926, near the old town, and it has hardly been changed on the outside, thanks to well thought-out renovation measures from the inside which ensure a new level of comfort and a minimization of the energy demand of the building.

House Moroder Bozen, IT
Building Period: 1900 - 1944 Renovation: 2015
Use: Residential (urban) Protection level: not listed
Contact:

EURAC Research 


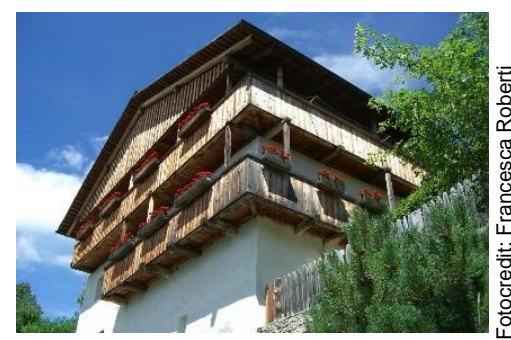

The farmhouse Huber is a listed building typical of the South Tyrolean countryside consisting of a main house and a barn. Given the poor conservation of the building, it went through an extensive refurbishment. The main goal was to adapt the house to modern standards while keeping unaltered its historic values and preserving it over time. Two apartments were created while preserving the old substance as much as possible. The barn was also renovated, rotten structural components were replaced. The farmhouse Huber was awarded as the best energy-efficient renovation in 2008.

\begin{tabular}{|llll|}
\hline $\begin{array}{l}\text { Huberhof } \\
\text { Rodeneck, IT }\end{array}$ & Building Period: before 1600 & Use: Residential (rural) & Contact: \\
Renovation: 2008 & Protection level: listed & EURAC Research \\
\hline
\end{tabular}

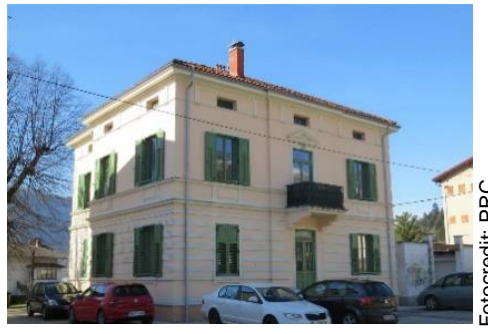

The house was built after the 1st world war as notary's villa in the former periphery of Tolmin where richer buildings were located. The house expresses the characteristics of secession and is listed in the Register of Slovene cultural heritage. Firstly, served as a single-family house, after the 2nd world war it was used for State security administration. Later it was rearranged to a four-apartment building. The earthquakes in 1998 and in 2004 caused severe damage on the building. It was therefore included in the national programme of renovation after the earthquake.

\begin{tabular}{|llll}
$\begin{array}{l}\text { Notarjeva Vila } \\
\text { Tolmin, SL }\end{array}$ & Building Period: $1900-1944$ & Use: Residential (urban) & Contact: \\
Renovation: 2015 & Protection level: listed & Posoški razvojni center
\end{tabular}

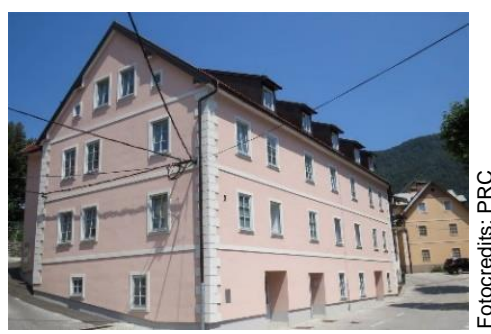

This building was constructed in the second half of the 19th century for the residence of mining families. It has several characteristics of the miner's house which is typical for the town of Idrija and represents very unique architecture. The building was in residential use until 2004, when it was so damaged by an earthquake that it was no longer habitable. Today, the ground floor is used for business premises and the upper floors are organized as apartments. For the needs of energy rehabilitation of the building, the external walls and roof were adequately insulated. Facade ornaments were made anew in accordance with the cultural heritage office demands.

\begin{tabular}{|llll|}
\hline $\begin{array}{l}\text { Rožna ulica 15 } \\
\text { Idrija, SL }\end{array}$ & Building Period: $1850-1899$ & Use: Residential (urban) & Contact: \\
& Renovation: 2018 & Protection level: listed & Posoški razvojni center \\
\hline
\end{tabular}

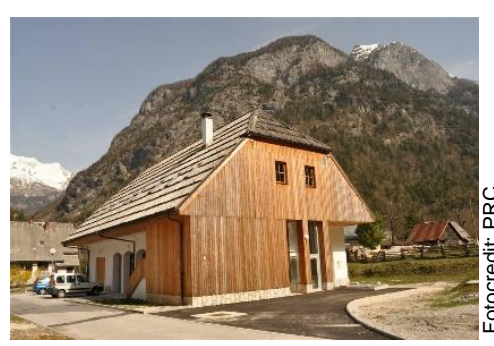

History of this building goes back to the early 30s of the 20th century when a new complex of the Cantore barracks was built by Italians in order to defend the Rapallo border. Within the renovation in 1999 the original structure was refurbished and upgraded in line with the local building typology. In 2012 the entire building was insulated and organised as a multipurpose house. The case shows how non typical architecture (former military barrack) can be renovated in a way that it gains traditional elements while following today's energy renovation standards.

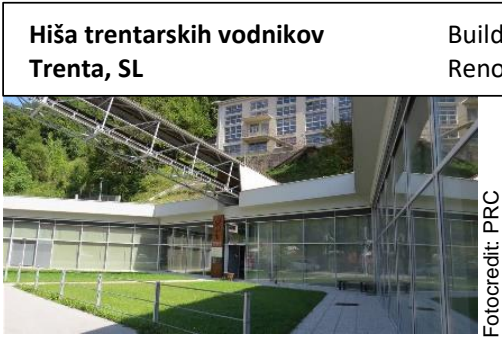

Build The diverse and unique industrial and technical heritage of Idrija's 500-year-old mining history was inscribed on UNESCO World Heritage List in 2012. One of the crucial parts of the mercury mine was the smelting plant, which stopped operating in 1995. The energy renovation of the building was carried out in accordance with the possibilities and specifications of the building, which belongs to the technical cultural heritage. Today the building serves as a museum with a visitor center that was additionally extended to the lower part of the smelting plant.

$\begin{array}{llll}\begin{array}{l}\text { Idrija mercury smelting plant } \\ \text { Idrija, SL }\end{array} & \text { Building Period: } 1945-1959 & \begin{array}{l}\text { Use: Educational/Research } \\ \text { Renovation: } 2017\end{array} & \begin{array}{l}\text { Contact: } \\ \text { Protection level: listed }\end{array} \\ \text { Posoški razvojni center }\end{array}$




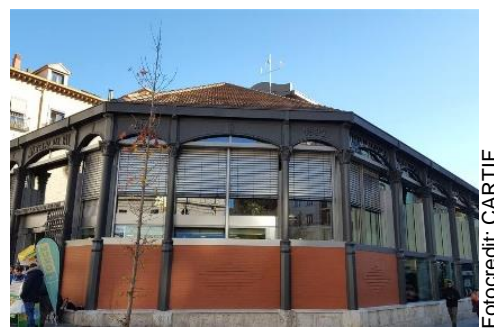

Mercado del Val is an iron market whose construction was completed in 1882 and it is located within the old town of Valladolid, Spain, being currently the oldest preserved market in the city. In 2013, the market was fully renovated recovering a late 19th century representative building of an architecture and commercial activity from that period, being respectful with its essence, but transforming it into an innovative building that meets the potentialities and commercial needs of the 21st century.

\begin{tabular}{|llll|}
\hline $\begin{array}{l}\text { Mercado del Val } \\
\text { Valladolid, ES }\end{array}$ & Building Period: $1850-1899$ & $\begin{array}{l}\text { Use: Wholesale \& Retail } \\
\text { Protection level: listed }\end{array}$ & $\begin{array}{l}\text { Contact: } \\
\text { CARTIF }\end{array}$ \\
\hline
\end{tabular}
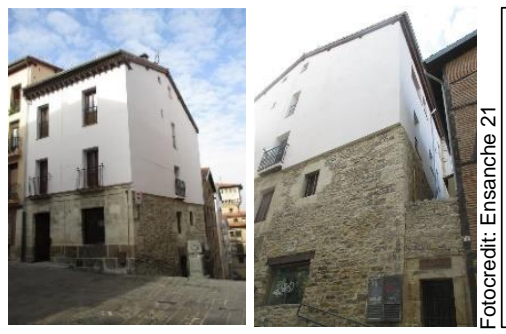

Building located at the historic centre of Vitoria-Gasteiz (Spain) that was constructed in 1886. It has a wooden structure and load-bearing façade walls of masonry and ashlar masonry and brick walls. It has been refurbished within the framework of the ENERPAT project where it was selected for being representative of the buildings of the historic district. It has basic protection due to being located in a protected area.

\begin{tabular}{|llll|}
\hline Correria 119 & Building Period: $1850-1899$ & Use: Residential (urban) & Contact: \\
Vitoria-Gasteiz, SP & Renovation: 2020 & Protection level: not listed & Tecnalia \\
\hline
\end{tabular}

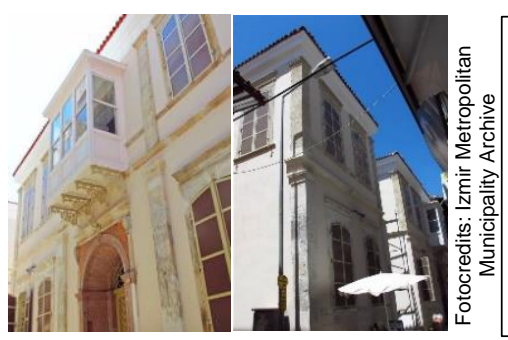

Ahmet Aga Mansion represents the typical interaction type of residence from the early 19th century of Izmir. The mansion was used for purposes other than housing by the late 19th century: firstly, as Gendarmerie School, then as Izmir Headquarters of Committee of Union and Progress and thirdly as the National Library. The building was abandoned and derelict for many years, until Izmir Metropolitan Municipality hired it to restore. The restoration was completed in 2013 and the mansion has been serving as the office of Directorate of Historical Environment and Cultural Properties.

\begin{tabular}{|llll|}
\hline $\begin{array}{l}\text { Ahmet Aga Mansion } \\
\text { Izmir, TK }\end{array}$ & Building Period: $1800-1849$ & Use: Offices & Contact: \\
Renovation: 2020 & Protection level: listed & Izmir Institute of Technology \\
\hline
\end{tabular}

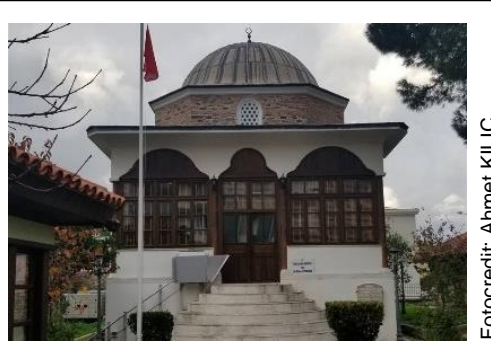

Necip Paşa Library was built in 1827 as an original library building by Necib Mehmed Pasha, an Ottoman statesman, to house his own book collection accumulated during his official service. Now, it contains 2718 rare manuscripts and printed books. The library, restored in 2017, provides the service to the researchers under the control and management of The Turkish Prime Ministry, Directorate General of Foundations.

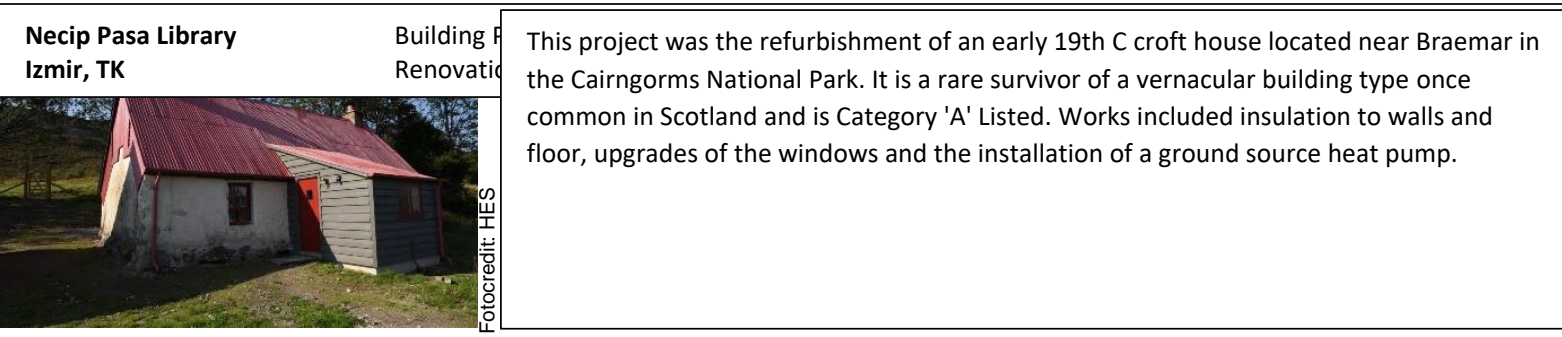

\begin{tabular}{|llll|}
\hline $\begin{array}{l}\text { Downie's Cottage } \\
\text { Braemar, GB }\end{array}$ & Building Period: $1800-1849$ & Use: Residential (rural) & Contact: \\
Renovation: 2016 & Protection level: listed & Historic Environment Scotland \\
\hline
\end{tabular}




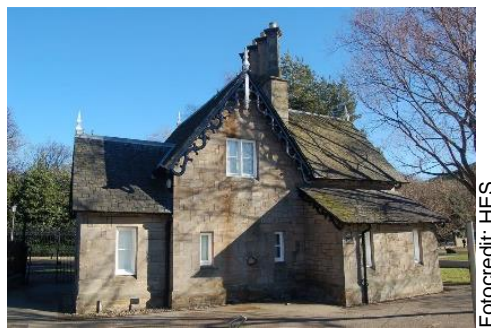

This project was the thermal upgrade and general improvements to a Listed property in Edinburgh dating from 1858. It was a whole house approach where the retention of historic fabric was a priority. Good results have been obtained and an improved energy rating (EPC) has been achieved. In addition, various traditional features have been repaired and reused for the buildings new use.

$\begin{array}{llll}\text { Holyrood Park Lodge } & \text { Building Period: } 1850-1899 & \text { Use: Wholesale \& Retail } & \text { Contact: } \\ \text { Edinburgh, GB } & \text { Renovation: } 2017 & \text { Protection level: listed } & \text { Historic Environment Scotland }\end{array}$

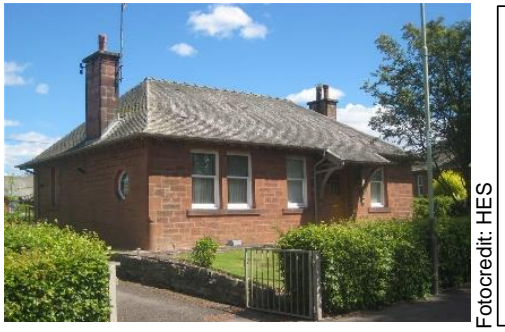

This project was the thermal upgrade of a traditional building in Perth dating to 1927 . This included improvements to the insulation of the walls, floor and roof as well as better natural ventilation. Natural and vapour open materials were prioritised. The project was a success and improved the $U$-values of individual elements considerably. This project was used to inform further refurbishment of other buildings on the estate.

\begin{tabular}{|c|c|c|}
\hline $\begin{array}{l}\text { Annat Road } \\
\text { Perth, GB }\end{array}$ & $\begin{array}{l}\text { Building Period: } 1900 \text { - } 1944 \\
\text { Renovation: } 2014\end{array}$ & $\begin{array}{l}\text { Use: Residential (urban) } \\
\text { Protection level: not listed }\end{array}$ \\
\hline
\end{tabular}

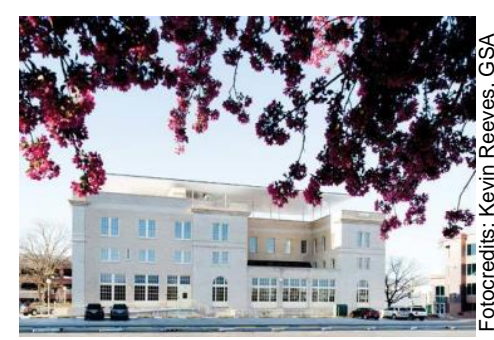

The US Federal government is mandated with improving efficiency of buildings, incorporating renewable energy, and achieving net-zero energy operations where possible. This challenge led GSA to consider aligning historic preservation renovations with net-zero energy goals. The agency chose the Wayne N. Aspinall Federal Building and U.S. Courthouse, to prove that net-zero energy goals can be achieved not only in an older building, but one that has a bevy of preservation requirements from being listed on the National Register of Historic Places.

$\begin{array}{llll}\text { Wayne N. Aspinall Courthouse } & \text { Building Period: } 1900-1944 & \text { Use: Offices } & \text { Contact: } \\ \text { Grand Junction, USA } & \text { Renovation: } 2013 & \text { Protection level: listed } & \text { Drexel University }\end{array}$




\subsection{Size of the buildings and building type}

The case studies are divided into large buildings, that are above 800 square meters gross floor area and small buildings with less than 800 square meters gross floor area (see Table 4).

The rural residential buildings are nearly all small buildings, except of one with $985 \mathrm{~m}^{2}$, whereas the urban residential buildings are more diverse in terms of their size, with 16 small buildings and 4 large buildings.

All other building types represented by the case studies have small as well as large examples, except of the religious buildings, which are only large buildings.

Overall, 55 case studies are small buildings and 14 case studies are large buildings.

Table 4 - Overview of Size

\begin{tabular}{|c|c|c|c|c|}
\hline No & Case study & Country & Size & Building type/use \\
\hline 1 & Klostergebäude Kaiserstrasse & AT & Large - $2750 \mathrm{~m}^{2}$ & Residential (urban) \\
\hline 2 & Farm house Trins & AT & Small - $698 \mathrm{~m}^{2}$ & Residential (rural) \\
\hline 3 & Hof 6, Schwarzenberg, Vorarlberg & AT & Small - $300 \mathrm{~m}^{2}$ & Residential (rural) \\
\hline 4 & Kelchalm - Bochumer alpine hut & AT & Small - $650 \mathrm{~m}^{2}$ & Hotel/Restaurant \\
\hline 5 & Mariahilferstrasse 182 & AT & Large - $3072 \mathrm{~m}^{2}$ & Residential (urban) \\
\hline 6 & House Maurer, Wolfurt & AT & Small - $370 \mathrm{~m}^{2}$ & Residential (rural) \\
\hline 7 & House Breuer, Tschagguns & AT & Small - $160 \mathrm{~m}^{2}$ & Residential (rural) \\
\hline 8 & Music school in Velden & AT & Small - $680 \mathrm{~m}^{2}$ & Educational/Research \\
\hline 9 & Hof NeuhäusI & AT & Small - $159 \mathrm{~m}^{2}$ & Residential (rural) \\
\hline 10 & Community Hall Zwischenwasser & AT & Small - 597 m² $^{2}$ & Community Hall \\
\hline 11 & Freihof Sulz & AT & Large $-1018 \mathrm{~m}^{2}$ & Restaurant/Commerce \\
\hline 12 & Oeconomy building Josef Weiss & AT & Small - $200 \mathrm{~m}^{2}$ & Residential with Atelier \\
\hline 13 & Giatla Haus & AT & Small - 236 m² $^{2}$ & Hotel/Restaurant \\
\hline 14 & Rhine Valley House Irgang & AT & Small - $180 \mathrm{~m}^{2}$ & Residential (rural) \\
\hline 15 & Baur Residence, Lustenau & AT & Small - 208 m² $^{2}$ & Residential (rural) \\
\hline 16 & Kasperhof & AT & Small - $650 \mathrm{~m}^{2}$ & Residential (rural) \\
\hline 17 & Maison Rubens & $\mathrm{BE}$ & Small - $239 \mathrm{~m}^{2}$ & Residential (urban) \\
\hline 18 & Half-timberframed house in Alken & BE & Small - $288 \mathrm{~m}^{2}$ & Residential (rural) \\
\hline 19 & Doragno Castle, Rovio & $\mathrm{CH}$ & Small - $490 \mathrm{~m}^{2}$ & Residential (rural) \\
\hline 20 & Solar silo & $\mathrm{CH}$ & Small - $606 \mathrm{~m}^{2}$ & Offices \\
\hline 21 & Wohn- und Geschäftshaus Feldbergstraße & $\mathrm{CH}$ & Small - 745 m² $^{2}$ & Residential (urban) \\
\hline 22 & Mehrfamilienhaus Magnusstrasse & $\mathrm{CH}$ & Large $-1040 \mathrm{~m}^{2}$ & Residential (urban) \\
\hline 23 & St. Franziskus Church Ebmatingen & $\mathrm{CH}$ & Large - $1420 \mathrm{~m}^{2}$ & Religious \\
\hline 24 & Kindergarten and apartments (PEB) Chur & $\mathrm{CH}$ & Small - $703 \mathrm{~m}^{2}$ & Educational/Research \\
\hline 25 & Single family home Luisenstrasse - Bern & $\mathrm{CH}$ & Small - $215 \mathrm{~m}^{2}$ & Residential (urban) \\
\hline 26 & Single Family House - Gstaad & $\mathrm{CH}$ & Small - $160 \mathrm{~m}^{2}$ & Residential (rural) \\
\hline 27 & Glaserhaus in Affoltern & $\mathrm{CH}$ & Large $-985 \mathrm{~m}^{2}$ & Residential (rural) \\
\hline 28 & PalaCinema Locarno & $\mathrm{CH}$ & Large $-5400 \mathrm{~m}^{2}$ & Platform for cinematic arts \\
\hline 29 & Casa Rossa Chemnitz & $\mathrm{DE}$ & Small - $578 \mathrm{~m}^{2}$ & Residential (urban) \\
\hline 30 & Rathaus Bergrheinfeld & DE & Small - $441 \mathrm{~m}^{2}$ & Town Hall \\
\hline 31 & Farmhouse Straub & $\mathrm{DE}$ & Small - $561 \mathrm{~m}^{2}$ & Residential (rural) \\
\hline 32 & Early work Sep Ruf & DE & Small - $216 \mathrm{~m}^{2}$ & Residential (rural) \\
\hline 33 & Ackerbürgerhäuschen & DE & Small - 208 m² $^{2}$ & Residential (rural) \\
\hline 34 & Ritterhof & $\mathrm{DE}$ & Small - $703 \mathrm{~m}^{2}$ & Residential (rural) \\
\hline 35 & Rathaus Burgkunstadt & DE & Small - $797 \mathrm{~m}^{2}$ & Town Hall \\
\hline 36 & Osramhuset (The Osram Building) & DK & Large - $824 \mathrm{~m}^{2}$ & Culture \& Community Centre \\
\hline 37 & Klitgaarden & DK & Small - $221 \mathrm{~m}^{2}$ & Residential (rural) \\
\hline
\end{tabular}




\begin{tabular}{|c|c|c|c|c|}
\hline 38 & RYESGADE 30 & DK & Large - $2760 \mathrm{~m}^{2}$ & Residential (urban) \\
\hline 39 & Timber-framed house in Alsace & FR & Small - $350 \mathrm{~m}^{2}$ & Residential (rural) \\
\hline 40 & Elementary School in Mulhouse & FR & Large - $1925 \mathrm{~m}^{2}$ & Education/Research \\
\hline 41 & Timber-framed barn in the north of France & $\mathrm{FR}$ & Small - $400 \mathrm{~m}^{2}$ & Residential (rural) \\
\hline 42 & Rainhof & IT & Small - $390 \mathrm{~m}^{2}$ & Residence (rural) \\
\hline 43 & Villa Castelli & IT & Small - $564 \mathrm{~m}^{2}$ & Residential (rural) \\
\hline 44 & Ansitz Kofler & IT & Small - $141 \mathrm{~m}^{2}$ & Residential (urban) \\
\hline 45 & Basilica di Santa Maria di Collemaggio & IT & Large $-2140 \mathrm{~m}^{2}$ & Religious \\
\hline 46 & House Pernter & IT & Small - $242 \mathrm{~m}^{2}$ & Residential (rural) \\
\hline 47 & Kohlerhaus & IT & Small - 195 m² $^{2}$ & Residential (urban) \\
\hline 48 & Ruckenzaunerhof & IT & Small - $267 \mathrm{~m}^{2}$ & Residential (rural) \\
\hline 49 & Aussergrubhof & IT & Small - $365 \mathrm{~m}^{2}$ & Residential (rural) \\
\hline 50 & Oberbergerhof & IT & Small - 134 m² $^{2}$ & Residential (rural) \\
\hline 51 & Platzbonhof & IT & Small - 369 m² $^{2}$ & Residential (rural) \\
\hline 52 & Mairhof & IT & Small - $770 \mathrm{~m}^{2}$ & Residential (rural) \\
\hline 53 & Obergasserhof & IT & Small - 369 m² $^{2}$ & Residential (rural) \\
\hline 54 & Rebecco Farm & IT & Small - $318 \mathrm{~m}^{2}$ & B\&B - Farm \\
\hline 55 & Villa Capodivacca & IT & Small - $748 \mathrm{~m}^{2}$ & Residential (urban) \\
\hline 56 & House Moroder & IT & Small - 268 m² $^{2}$ & Residential (urban) \\
\hline 57 & Huberhof & IT & Small - 394 m² $^{2}$ & Residential (rural) \\
\hline 58 & Notarjeva vila & SL & Small - $412 \mathrm{~m}^{2}$ & Residential (urban) \\
\hline 59 & Rožna ulica 15, Idrija & SL & Small - $793 \mathrm{~m}^{2}$ & Residential (urban) \\
\hline 60 & Hiša trentarskih vodnikov & SL & Small - $514 \mathrm{~m}^{2}$ & Multipurpose \\
\hline 61 & Idrija mercury smelting plant & SL & Large - - $\mathrm{m}^{2}$ & Educational/Research \\
\hline 62 & Mercado del Val, Valladolid & SP & Large - $3936 \mathrm{~m}^{2}$ & Wholesale and Retail \\
\hline 63 & Correria 119 & SP & Small - $371 \mathrm{~m}^{2}$ & Residential (urban) \\
\hline 64 & Ahmet Aga Mansion & TK & Small - $460 \mathrm{~m}^{2}$ & Offices \\
\hline 65 & Nwcip Pasa Library & TK & Small - $66 \mathrm{~m}^{2}$ & Library \\
\hline 66 & Downie's Cottage & UK & Small - $20 \mathrm{~m}^{2}$ & Residential (rural) \\
\hline 67 & Hollyrood Park Lodge & UK & Small - $70 \mathrm{~m}^{2}$ & Wholesale and Retail \\
\hline 68 & Annat Road & UK & Small - $77 \mathrm{~m}^{2}$ & Residential (urban) \\
\hline 69 & Aspinall Courthouse & USA & Large - $3900 \mathrm{~m}^{2}$ & Offices \\
\hline
\end{tabular}




\subsection{Level of protection}

The main categories regarding levels of protection are listed or protected buildings and non-listed buildings. A further distinction was made in case the buildings are situated in a protected area (see Table 5).

Listed are 31 of the 69 case studies, protected are 34 buildings. Situated in a protected area are 24 buildings.

Table 5 - Overview on building period and the year of the last renovation

\begin{tabular}{|c|c|c|c|c|c|c|}
\hline No & Case study & 竞 & 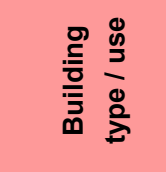 & 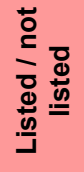 & 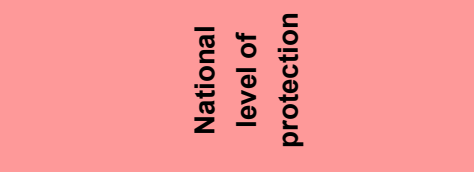 & 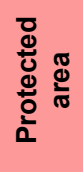 \\
\hline 1 & Klostergebäude Kaiserstrasse & AT & Res / urban & $x$ & Monument protection & - \\
\hline 2 & Farm house Trins & AT & Res / rural & - & - & - \\
\hline 3 & Hof 6, Schwarzenberg, Vorarlberg & AT & Res / rural & $x$ & $\begin{array}{l}\text { The former main-/living part of the } \\
\text { house has a heritage protection }\end{array}$ & $\mathrm{x}$ \\
\hline 4 & Kelchalm - Bochumer alpine hut & AT & Hotel/Rest & - & - & - \\
\hline 5 & Mariahilferstrasse 182 & AT & Res / urban & - & - & - \\
\hline 6 & House Maurer, Wolfurt & AT & Res / rural & - & "Rhine valley houses" & $\mathrm{x}$ \\
\hline 7 & House Breuer, Tschagguns & AT & Res / rural & - & - & - \\
\hline 8 & Music school in Velden & AT & Educational & $x$ & DMSG $\S 2 a$ & - \\
\hline 9 & Hof NeuhäusI & AT & Res / rural & - & - & - \\
\hline 10 & Community Hall Zwischenwasser & AT & $\begin{array}{l}\text { Community } \\
\text { Hall }\end{array}$ & - & - & - \\
\hline 11 & Freihof Sulz & AT & Multipurpos & $x$ & - & - \\
\hline 12 & Oeconomy building Josef Weiss & AT & $\begin{array}{l}\text { Res + } \\
\text { Atelier }\end{array}$ & $x$ & - & - \\
\hline 13 & Giatla Haus & AT & Hotel/Rest & - & - & - \\
\hline 14 & Rhine Valley House Irgang & AT & Res / rural & - & - & - \\
\hline 15 & Baur Residence, Lustenau & AT & Res / rural & - & - & - \\
\hline 16 & Kasperhof & AT & Res / rural & - & Protection of built Landscapes & $x$ \\
\hline 17 & Maison Rubens & $\mathrm{BE}$ & Res / urban & - & - & - \\
\hline 18 & Half-timberframed house in Alken & BE & Res / rural & $\mathrm{x}$ & Protected Monument & - \\
\hline 19 & Doragno Castle, Rovio & $\mathrm{CH}$ & Res / rural & - & - & - \\
\hline 20 & Solar silo & $\mathrm{CH}$ & Offices & - & $\begin{array}{l}\text { "Grundelinger Feld" as an ensemble } \\
\text { is under heritage protection }\end{array}$ & $\mathrm{x}$ \\
\hline 21 & $\begin{array}{l}\text { Wohn- und Geschäftshaus } \\
\text { Feldbergstraße }\end{array}$ & $\mathrm{CH}$ & Res / urban & - & $\begin{array}{l}\text { "Schonzone" of Basel-Stadt, with } \\
\text { aesthetic requirements }\end{array}$ & $x$ \\
\hline 22 & Mehrfamilienhaus Magnusstrasse & $\mathrm{CH}$ & Res / urban & $\mathrm{x}$ & $\begin{array}{l}\text { The two street-side facades are } \\
\text { subject to preservation requirements }\end{array}$ & $\mathrm{x}$ \\
\hline 23 & St. Franziskus Church Ebmatingen & $\mathrm{CH}$ & Religious & - & - & - \\
\hline 24 & Kindergarten and apartments (PEB) & $\mathrm{CH}$ & Educational & - & - & - \\
\hline 25 & $\begin{array}{l}\text { Single family House Luisenstrasse - } \\
\text { Bern }\end{array}$ & $\mathrm{CH}$ & Res / urban & $x$ & "high" & $\mathrm{x}$ \\
\hline 26 & Single Family House - Gstaad & $\mathrm{CH}$ & Res / rural & $\mathrm{X}$ & $\begin{array}{l}\text { Federal legal restrictions on the } \\
\text { preservation of cultural landscapes }\end{array}$ & $\mathrm{X}$ \\
\hline 27 & Glaserhaus in Affoltern & $\mathrm{CH}$ & Res / rural & $x$ & $\begin{array}{l}\text { Protected at cantonal level (Canton } \\
\text { Bern) }\end{array}$ & - \\
\hline 28 & PalaCinema Locarno & $\mathrm{CH}$ & Cultural & - & A (maximum level of protection) & $x$ \\
\hline 29 & Casa Rossa Chemnitz & $\mathrm{DE}$ & Res / urban & - & - & $x$ \\
\hline 30 & Rathaus Bergrheinfeld & $\mathrm{DE}$ & Town Hall & $x$ & - & - \\
\hline
\end{tabular}




\begin{tabular}{|c|c|c|c|c|c|c|}
\hline 31 & Farmhouse Straub & $\mathrm{DE}$ & Res / rural & - & - & - \\
\hline 32 & Early work Sep Ruf & $\mathrm{DE}$ & Res / rural & $x$ & - & - \\
\hline 33 & Ackerbürgerhäuschen & $\mathrm{DE}$ & Res / rural & $x$ & - & $x$ \\
\hline 34 & Ritterhof & $\mathrm{DE}$ & Res / rural & - & - & - \\
\hline 35 & Rathaus Burgkunstadt & $\mathrm{DE}$ & Townhall & $x$ & - & $x$ \\
\hline 36 & Osramhuset (The Osram Building) & DK & $\begin{array}{l}\text { Comm } \\
\text { Centre }\end{array}$ & $x$ & The façade is worthy of preservation & - \\
\hline 37 & Klitgaarden & DK & Res / rural & - & - & - \\
\hline 38 & RYESGADE 30 & DK & Res / urban & $x$ & $\begin{array}{l}\text { SAVE IVl } 4 \text { - façade cannot be } \\
\text { changed }\end{array}$ & - \\
\hline 39 & Timber-framed house in Alsace & FR & Res / rural & - & - & - \\
\hline 40 & Elementary School in Mulhouse & $\mathrm{FR}$ & Educational & $x$ & $\begin{array}{l}\text { The street-facing façade, the roof } \\
\text { and two inner staircases are } \\
\text { classified on the French supple- } \\
\text { mentary historic monument list }\end{array}$ & - \\
\hline 41 & $\begin{array}{l}\text { Timber-framed barn in the north of } \\
\text { France }\end{array}$ & FR & Res / rural & - & - & - \\
\hline 42 & Rainhof & IT & Res / rural & $x$ & $\begin{array}{l}\text { Constraint Provision: BLR-LAB } 607 \\
\text { from 16/02/1987 }\end{array}$ & - \\
\hline 43 & Villa Castelli & IT & Res / rural & $x$ & BLR-LAB Number and Date missing & $x$ \\
\hline 44 & Ansitz Kofler & IT & Res / urban) & $x$ & $\begin{array}{l}\text { Constraint Provision BLR-LAB } 4980 \\
\text { from 25/07/1977 }\end{array}$ & $\mathrm{x}$ \\
\hline 45 & $\begin{array}{l}\text { Basilica di Santa Maria di } \\
\text { Collemaggio }\end{array}$ & IT & Religious & $x$ & $\begin{array}{l}\text { National Monument - BLR-LAB } \\
\text { Number and Date missing }\end{array}$ & $\mathrm{x}$ \\
\hline 46 & House Pernter & IT & Res / rural & - & - & - \\
\hline 47 & Kohlerhaus & IT & Res / urban & - & Ensemble protection South Tyrol & $x$ \\
\hline 48 & Ruckenzaunerhof & IT & Res / rural & $x$ & $\begin{array}{l}\text { Protected monument - BRL-LAB } \\
\text { Number and Date missing }\end{array}$ & - \\
\hline 49 & Aussergrubhof & IT & Res / rural & - & - & - \\
\hline 50 & Oberbergerhof & IT & Res / rural & $x$ & $\begin{array}{l}\text { Protected monument - BRL-LAB } \\
\text { Number and Date missing }\end{array}$ & - \\
\hline 51 & Platzbonhof & IT & Res / rural & - & - & - \\
\hline 52 & Mairhof & IT & Res / rural & $x$ & BRL-LAB Number and Date missing & $\mathrm{x}$ \\
\hline 53 & Obergasserhof & IT & Res / rural & $x$ & $\begin{array}{l}\text { Protected monument - BRL-LAB } \\
\text { Number and Date missing }\end{array}$ & - \\
\hline 54 & Rebecco Farm & IT & B\&B - Farm & - & Landscape Restriction & $x$ \\
\hline 55 & Villa Capodivacca & IT & Res / urban & $x$ & $\begin{array}{l}\text { Decreto di vincolo 6/2/1076 ai sensi } \\
\text { della legge } 1089 / 39 \text { - DLgs } 42 / 2004\end{array}$ & $x$ \\
\hline 56 & House Moroder & IT & Res / urban & - & - & - \\
\hline 57 & Huberhof & IT & Res / rural & $x$ & $\begin{array}{l}\text { Architectural protection DGP-LAB } \\
504107 / 10 / 1985\end{array}$ & - \\
\hline 58 & Notarjeva vila & SL & Res / urban & $x$ & $\begin{array}{l}\text { Listed in national register of cultural } \\
\text { heritage }\end{array}$ & - \\
\hline 59 & Rožna ulica 15 , Idrija & $\mathrm{SL}$ & Res / urban & $x$ & Heritage of local importance & $\mathrm{x}$ \\
\hline 60 & Hiša trentarskih vodnikov & SL & Multipurpos & - & National park & $x$ \\
\hline 61 & Idrija mercury smelting plant & SL & Educational & $x$ & $\begin{array}{l}\text { UNESCO World Heritage List, } \\
\text { cultural monument of national } \\
\text { importance }\end{array}$ & $x$ \\
\hline 62 & Mercado del Val, Valladolid & $\mathrm{SP}$ & Retail & $x$ & Classified as P3 (art. 5.2.7 PECH) & $x$ \\
\hline 63 & Correria 119 & $\mathrm{SP}$ & Res / urban & - & "Basic" & $\mathrm{x}$ \\
\hline 64 & Ahmet Aga Mansion & TK & Offices & $x$ & $\begin{array}{l}2^{\text {nd }} \text { degree of cultural property in } \\
\text { need of protection / civil architecture } \\
\text { example }\end{array}$ & $x$ \\
\hline 65 & Nwcip Pasa Library & TK & Library & $x$ & Monumental & $\mathrm{x}$ \\
\hline 66 & Downie's Cottage & UK & Res / rural & $x$ & $\begin{array}{l}\text { Scottish Category A - denoting } \\
\text { national importance }\end{array}$ & $\mathrm{x}$ \\
\hline
\end{tabular}




\begin{tabular}{|l|l|l|l|l|l|l|}
\hline 67 & Holyrood Park Lodge & UK & Retail & X & $\begin{array}{l}\text { Scottish Category B - regionally } \\
\text { important }\end{array}$ & X \\
\hline 68 & Annat Road & UK & Res / urban & - & - & - \\
\hline 69 & Aspinall Courthouse & USA & Offices & X & National Register of Historic Places & X \\
\hline
\end{tabular}

\subsection{Building period and year of renovation}

The case studies are divided according to the period of their construction. This is useful to determine their age, their state of repair and the technologies that were used in their construction. Also, the year of the last renovation, and, if available, the date of previous renovations is identified.

The range of this categorization is from before 1600 (14 case studies), 1600-1700 (7 case studies), $1700-1800$ (9 case studies), 1800-1849 (5 case studies), 1850-1899 (19 case studies), 1900-1944 (12 cases studies), to 1945 1959 ( 2 case studies) to 1980-present ( 1 case study). Table 6 gives an overview on the building periods of the case studies and the year of the latest renovation.

In the period before 1600, there is an emphasis on South Tyrolean (farm) houses. Between 1600 and 1800 (former) Austrian farm houses are dominant. In the period from 1850-1899, primarily urban buildings (with different use) were analysed. The rest of the case studies are evenly spread in the other periods.

Most of the renovations (56 case studies) took place since 2013.

Table 6 - Overview on building period and the year of the last renovation

\begin{tabular}{|c|c|c|c|c|c|}
\hline No & Case study & 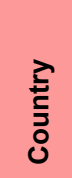 & 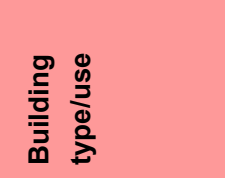 & 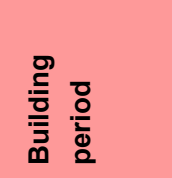 & 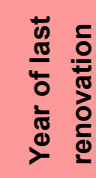 \\
\hline 1 & Klostergebäude Kaiserstrasse & AT & Res / urban & 1850-1899 & 2013 \\
\hline 2 & Farm house Trins & AT & Res / rural & $1600-1700$ & 2019 \\
\hline 3 & Hof 6, Schwarzenberg, Vorarlberg & AT & Res / rural & $1600-1700$ & 2013 \\
\hline 4 & Kelchalm - Bochumer alpine hut & AT & Hotel/Rest & $1800-1849$ & 2013 \\
\hline 5 & Mariahilferstrasse 182 & AT & Res / urban & 1850-1899 & 2018 \\
\hline 6 & House Maurer, Wolfurt & AT & Res / rural & $1800-1849$ & 2015 \\
\hline 7 & House Breuer, Tschagguns & AT & Res / rural & $1900-1944$ & 2015 \\
\hline 8 & Music school in Velden & AT & Educational & $1900-1944$ & 2015 \\
\hline 9 & Hof Neuhäusl & AT & Res / rural & $1700-1800$ & 2017 \\
\hline 10 & Community Hall Zwischenwasser & AT & Community Hall & $1900-1944$ & 2015 \\
\hline 11 & Freihof Sulz & AT & Multipurpose & $1700-1800$ & 2006 \\
\hline 12 & Oeconomy building Josef Weiss & AT & Res + Atelier & 1850-1899 & 2016 \\
\hline 13 & Giatla Haus & AT & Hotel/Rest & $1600-1700$ & 2015 \\
\hline 14 & Rhine Valley House Irgang & AT & Res / rural & 1850-1899 & 2008 \\
\hline 15 & Baur Residence, Lustenau & AT & Res / rural & 1850-1899 & 2011 \\
\hline 16 & Kasperhof & AT & Res / rural & $1600-1700$ & 2019 \\
\hline 17 & Maison Rubens & $\mathrm{BE}$ & Res / urban & 1850-1899 & 2008 \\
\hline 18 & Half-timberframed house in Alken & $\mathrm{BE}$ & Res / rural & $1600-1700$ & 2016 \\
\hline 19 & Doragno Castle, Rovio & $\mathrm{CH}$ & Res / rural & before 1600 & 2017 \\
\hline 20 & Solar silo & $\mathrm{CH}$ & Offices & $1850-1899$ & 2014 \\
\hline 21 & $\begin{array}{l}\text { Wohn- und Geschäftshaus } \\
\text { Feldbergstraße }\end{array}$ & $\mathrm{CH}$ & Res / urban & 1850-1899 & 2009 \\
\hline 22 & Mehrfamilienhaus Magnusstrasse & $\mathrm{CH}$ & Res / urban & 1850-1899 & 2007 \\
\hline
\end{tabular}




\begin{tabular}{|c|c|c|c|c|c|}
\hline 23 & St. Franziskus Church Ebmatingen & $\mathrm{CH}$ & Religious & 1980-present & 2018 \\
\hline 24 & Kindergarten and apartments (PEB) & $\mathrm{CH}$ & Educational & $1900-1944$ & 2016 \\
\hline 25 & Single family home Luisenstrasse & $\mathrm{CH}$ & Res / urban & $1850-1899$ & 2015 \\
\hline 26 & Single Family House - Gstaad & $\mathrm{CH}$ & Res / rural & $1700-1800$ & 2018 \\
\hline 27 & Glaserhaus in Affoltern & $\mathrm{CH}$ & Res / rural & $1700-1800$ & 2015 \\
\hline 28 & PalaCinema Locarno & $\mathrm{CH}$ & Cultural & $1850-1899$ & 2017 \\
\hline 29 & Casa Rossa Chemnitz & DE & Res / urban & $1900-1944$ & 2018 \\
\hline 30 & Rathaus Bergrheinfeld & DE & Town Hall & $1600-1700$ & 2018 \\
\hline 31 & Farmhouse Straub & $\mathrm{DE}$ & Res / rural & $1700-1800$ & 2018 \\
\hline 32 & Early work Sep Ruf & DE & Res / rural & $1900-1944$ & 2014 \\
\hline 33 & Ackerbürgerhäuschen & DE & Res / rural & before 1600 & 2015 \\
\hline 34 & Ritterhof & DE & Res / rural & $1850-1899$ & 2016 \\
\hline 35 & Rathaus Burgkunstadt & DE & Townhall & before 1600 & 2009 \\
\hline 36 & Osramhuset (The Osram Building) & DK & Comm Centre & $1945-1959$ & 2009 \\
\hline 37 & Klitgaarden & DK & Res / rural & $1850-1899$ & 2016 \\
\hline 38 & RYESGADE 30 & DK & Res / urban & $1850-1899$ & 2011 \\
\hline 39 & Timber-framed house in Alsace & $\mathrm{FR}$ & Res / rural & $1700-1800$ & 2015 \\
\hline 40 & Elementary School in Mulhouse & FR & Educational & $1700-1800$ & 2015 \\
\hline 41 & $\begin{array}{l}\text { Timber-framed barn in the north of } \\
\text { France }\end{array}$ & FR & Res / rural & $1700-1800$ & 2016 \\
\hline 42 & Rainhof & IT & Res / rural & before 1600 & 2016 \\
\hline 43 & Villa Castelli & IT & Res / rural & $1850-1899$ & 2013 \\
\hline 44 & Ansitz Kofler & IT & Res / urban) & $1700-1800$ & 2008 \\
\hline 45 & Basilica di Santa Maria di Collemaggio & IT & Religious & before 1600 & 2017 \\
\hline 46 & House Pernter & IT & Res / rural & $1900-1944$ & 2017 \\
\hline 47 & Kohlerhaus & IT & Res / urban & before 1600 & 2011 \\
\hline 48 & Ruckenzaunerhof & IT & Res / rural & before 1600 & 2015 \\
\hline 49 & Aussergrubhof & IT & Res / rural & $1600-1700$ & 2014 \\
\hline 50 & Oberbergerhof & IT & Res / rural & before 1600 & 2016 \\
\hline 51 & Platzbonhof & IT & Res / rural & before 1600 & 2016 \\
\hline 52 & Mairhof & IT & Res / rural & before 1600 & 2018 \\
\hline 53 & Obergasserhof & IT & Res / rural & before 1600 & 2013 \\
\hline 54 & Rebecco Farm & IT & B\&B - Farm & before 1600 & $\sim 2017$ \\
\hline 55 & Villa Capodivacca & IT & Res / urban & before 1600 & 2017 \\
\hline 56 & House Moroder & IT & Res / urban & $1900-1944$ & 2015 \\
\hline 57 & Huberhof & IT & Res / rural & before 1600 & 2008 \\
\hline 58 & Notarjeva vila & SL & Res / urban & $1900-1944$ & 2015 \\
\hline 59 & Rožna ulica 15, Idrija & SL & Res / urban & $1850-1899$ & 2018 \\
\hline 60 & Hiša trentarskih vodnikov & SL & Multipurpose & $1900-1944$ & 2012 \\
\hline 61 & Idrija mercury smelting plant & SL & Educational & $1945-1959$ & 2017 \\
\hline 62 & Mercado del Val, Valladolid & SP & Retail & $1850-1899$ & 2016 \\
\hline 63 & Correria 119 & SP & Res / urban & $1850-1899$ & 2020 \\
\hline 64 & Ahmet Aga Mansion & TK & Offices & $1800-1849$ & 2020 \\
\hline 65 & Nwcip Pasa Library & TK & Library & $1800-1849$ & 2017 \\
\hline 66 & Downie's Cottage & UK & Res / rural & $1800-1849$ & 2016 \\
\hline 67 & Holyrood Park Lodge & UK & Retail & $1850-1899$ & 2017 \\
\hline 68 & Annat Road & UK & Res / urban & $1900-1944$ & 2014 \\
\hline 69 & Aspinall Courthouse & USA & Offices & $1900-1944$ & 2013 \\
\hline
\end{tabular}




\subsection{Specifics of changes to the building}

The specifics of changes to the buildings were analysed to determine if it was a refurbishment or a retrofit, or if extensions or some other type of modification were made to the building. Overall, 18 extensions and 32 transformations were made in the 69 case studies (see Table 7).

Extensions primarily included attic space (4 case studies), and adding living space (6 case studies).

Most transformations (16 case studies) concerned agricultural buildings (partly including residential use) which became mainly residential (including apartments, B\&B, ....). There is also a group of 5 case studies in which private residential buildings were transformed into apartment buildings. In 9 cases a more substantial transformation especially in the use of the building took place:

- The music school in Velden used to be a fire station.

- Doragno Castle was transformed from a medieval ruin to a private residence.

- The solar silo, a former coal silo, is now a multi-purpose office building.

- Kindergarten and apartments (PEB) Chur used to be a public space and are now residential/educational.

- The PalaCinema Locarno was transformed form a school into a cultural building.

- Osramhuset was an industrial building and is now a cultural building.

- Idrija mercury smelting plant was converted to a museum with a visitor center.

- Hiša trentarskih vodnikov was a military barrack used as a kitchen and dining place and is now a multipurpose educational building.

- Ahmet Aga Mansion was in its origin a residential building, meanwhile serving as a gendarmerie school, headquarters for a political party, and national library, but was before its refurbishment an abandoned ruin. Now it is used as a municipal office building.

- Farmhouse Trins had a large part, that was used for holding sheep, but is now a living space.

Table 7 - Overview on the projects, the categories and the commentary

\begin{tabular}{|c|c|c|c|c|c|c|}
\hline No & Case study & 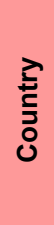 & 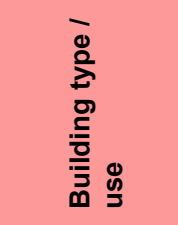 & 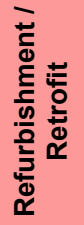 & $\begin{array}{l}\frac{c}{0} \\
\frac{0}{d} \\
\frac{x}{x} \\
\text { ய }\end{array}$ & 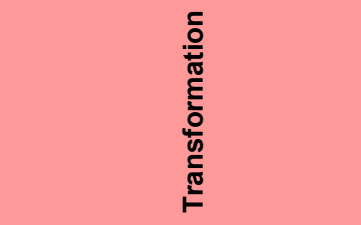 \\
\hline 1 & Klostergebäude Kaiserstrasse & AT & Res / urban & $x$ & $\begin{array}{l}\text { Extension of the } \\
\text { attic }\end{array}$ & - \\
\hline 2 & Farm house Trins & AT & Res / rural & $x$ & $\begin{array}{l}\text { House-in-house } \\
\text { concept }\end{array}$ & $\begin{array}{l}\text { Transformation from a } \\
\text { former sheep stable into } \\
\text { residential space }\end{array}$ \\
\hline 3 & $\begin{array}{l}\text { Hof 6, Schwarzenberg, } \\
\text { Vorarlberg }\end{array}$ & AT & Res / rural & $x$ & $\begin{array}{l}\text { Extension of the } \\
\text { living area to the } \\
\text { former barn }\end{array}$ & - \\
\hline 4 & $\begin{array}{l}\text { Kelchalm - Bochumer alpine } \\
\text { hut }\end{array}$ & AT & Hotel/Rest & $x$ & - & - \\
\hline 5 & Mariahilferstrasse 182 & AT & Res / urban & $x$ & $\begin{array}{l}\text { Added two-storey } \\
\text { attic with } 9 \\
\text { apartments }\end{array}$ & - \\
\hline 6 & House Maurer, Wolfurt & AT & Res / rural & $x$ & - & - \\
\hline 7 & House Breuer, Tschagguns & AT & Res / rural & $x$ & - & $\begin{array}{l}\text { Transformation from an } \\
\text { agricultural building to a } \\
\text { high-quality residential } \\
\text { building }\end{array}$ \\
\hline 8 & Music school in Velden & AT & Educational & $x$ & - & $\begin{array}{l}\text { Transformation from a } \\
\text { former fire station to a music } \\
\text { school }\end{array}$ \\
\hline 9 & Hof Neuhäusl & AT & Res / rural & $x$ & - & $\begin{array}{l}\text { Transformation of the former } \\
\text { barn to a garage with } \\
\text { storage and technical room }\end{array}$ \\
\hline 10 & $\begin{array}{l}\text { Community Hall } \\
\text { Zwischenwasser }\end{array}$ & AT & $\begin{array}{l}\text { Community } \\
\text { Hall }\end{array}$ & $x$ & - & - \\
\hline 11 & Freihof Sulz & AT & Multipurpose & $x$ & - & $\begin{array}{l}\text { Transformation from a } \\
\text { residential house to a "lively } \\
\text { meeting place" }\end{array}$ \\
\hline
\end{tabular}




\begin{tabular}{|c|c|c|c|c|c|c|}
\hline 12 & $\begin{array}{l}\text { Oeconomy building Josef } \\
\text { Weiss }\end{array}$ & AT & Res + Atelier & $x$ & - & - \\
\hline 13 & Giatla Haus & AT & Hotel/Rest & $x$ & - & $\begin{array}{l}\text { Farmhouse was converted } \\
\text { into an apartment house }\end{array}$ \\
\hline 14 & Rhine Valley House Irgang & AT & Res / rural & $x$ & - & - \\
\hline 15 & Baur Residence, Lustenau & AT & Res / rural & $x$ & $\begin{array}{l}\text { The utility wing in } \\
\text { the northern part } \\
\text { of the building was } \\
\text { extended }\end{array}$ & $\begin{array}{l}\text { Former non-living area is } \\
\text { now used as living area. }\end{array}$ \\
\hline 16 & Kasperhof & AT & Res / rural & $x$ & - & $\begin{array}{l}\text { Transformation from a farm } \\
\text { house to residential use and } \\
\text { offices. }\end{array}$ \\
\hline 17 & Maison Rubens & $\mathrm{BE}$ & Res / urban & $\mathrm{X}$ & - & - \\
\hline 18 & $\begin{array}{l}\text { Half-timberframed house in } \\
\text { Alken }\end{array}$ & $\mathrm{BE}$ & Res / rural & $x$ & - & - \\
\hline 19 & Doragno Castle, Rovio & $\mathrm{CH}$ & Res / rural & $x$ & - & $\begin{array}{l}\text { Transformation of a } \\
\text { medieval castle into a } \\
\text { private residence }\end{array}$ \\
\hline 20 & Solar silo & $\mathrm{CH}$ & Offices & $\mathrm{X}$ & - & $\begin{array}{l}\text { Transformation of a coal silo } \\
\text { into a multi-purpose building }\end{array}$ \\
\hline 21 & $\begin{array}{l}\text { Wohn- und Geschäftshaus } \\
\text { Feldbergstraße }\end{array}$ & $\mathrm{CH}$ & Res / urban & $x$ & - & - \\
\hline 22 & $\begin{array}{l}\text { Mehrfamilienhaus } \\
\text { Magnusstrasse }\end{array}$ & $\mathrm{CH}$ & Res / urban & $x$ & $\begin{array}{l}\text { The roof was } \\
\text { raised on the } \\
\text { courtyard side to } \\
\text { create a new floor }\end{array}$ & - \\
\hline 23 & $\begin{array}{l}\text { St. Franziskus Church } \\
\text { Ebmatingen }\end{array}$ & $\mathrm{CH}$ & Religious & $x$ & - & - \\
\hline 24 & $\begin{array}{l}\text { Kindergarten and apartments } \\
\text { (PEB) Chur }\end{array}$ & $\mathrm{CH}$ & Educational & $x$ & - & $\begin{array}{l}\text { The building was turned into } \\
\text { two Kindergarten spaces } \\
\text { (ground storey) and } \\
\text { residential area (upper floor) }\end{array}$ \\
\hline 25 & $\begin{array}{l}\text { Single family home } \\
\text { Luisenstrasse - Bern }\end{array}$ & $\mathrm{CH}$ & Res / urban & $x$ & - & - \\
\hline 26 & Single Family House - Gstaad & $\mathrm{CH}$ & Res / rural & $x$ & $\begin{array}{l}\text { Extension of the } \\
\text { basement floor }\end{array}$ & $\begin{array}{l}\text { Transformation from } \\
\text { agricultural to residential use }\end{array}$ \\
\hline 27 & Glaserhaus in Affoltern & $\mathrm{CH}$ & Res / rural & $x$ & - & - \\
\hline 28 & PalaCinema Locarno & $\mathrm{CH}$ & Cultural & $x$ & - & $\begin{array}{l}\text { Transformation from a } \\
\text { former school to a cinema } \\
\text { and cultural centre }\end{array}$ \\
\hline 29 & Casa Rossa Chemnitz & $\mathrm{DE}$ & Res / urban & $x$ & - & - \\
\hline 30 & Rathaus Bergrheinfeld & $\mathrm{DE}$ & Town Hall & $x$ & $\begin{array}{l}\text { Extension of the } \\
\text { building with a } \\
\text { textile façade }\end{array}$ & - \\
\hline 31 & Farmhouse Straub & $\mathrm{DE}$ & Res / rural & $x$ & & $\begin{array}{l}\text { Transformation from a farm } \\
\text { house to a residential } \\
\text { building }\end{array}$ \\
\hline 32 & Early work by Sep Ruf & $\mathrm{DE}$ & Res / rural & $x$ & - & - \\
\hline 33 & Ackerbürgerhäuschen & $\mathrm{DE}$ & Res / rural & $x$ & - & - \\
\hline 34 & Ritterhof & $\mathrm{DE}$ & Res / rural & $x$ & - & $\begin{array}{l}\text { Transformation from a farm } \\
\text { house to a multi-purpose } \\
\text { use: office, flat, shop }\end{array}$ \\
\hline 35 & Rathaus Burgkunstadt & $\mathrm{DE}$ & Townhall & $x$ & - & - \\
\hline 36 & $\begin{array}{l}\text { Osramhuset (The Osram } \\
\text { Building) }\end{array}$ & DK & Comm Centre & $x$ & - & $\begin{array}{l}\text { Transformation of a former } \\
\text { industrial building into a } \\
\text { community centre }\end{array}$ \\
\hline 37 & Klitgaarden & DK & Res / rural & $x$ & - & - \\
\hline 38 & RYESGADE 30 & DK & Res / urban & $x$ & - & - \\
\hline 39 & $\begin{array}{l}\text { Timber-framed house in } \\
\text { Alsace }\end{array}$ & FR & Res / rural & $x$ & - & - \\
\hline
\end{tabular}




\begin{tabular}{|c|c|c|c|c|c|c|}
\hline 40 & $\begin{array}{l}\text { Elementary School in } \\
\text { Mulhouse }\end{array}$ & $\mathrm{FR}$ & Educational & $\mathrm{X}$ & $\begin{array}{l}\text { Additional building } \\
\text { for canteen and } \\
\text { sports hall }\end{array}$ & - \\
\hline 41 & $\begin{array}{l}\text { Timber-framed barn in the } \\
\text { north of France }\end{array}$ & $\mathrm{FR}$ & Res / rural & $\mathrm{x}$ & - & $\begin{array}{l}\text { Transformation from a hay } \\
\text { barn to a residential building }\end{array}$ \\
\hline 42 & Rainhof & $\mathrm{IT}$ & Res / rural & $x$ & $\begin{array}{l}\text { Extension on the } \\
\text { north of the house }\end{array}$ & $\begin{array}{l}\text { Transformation from an } \\
\text { agricultural and living use to } \\
\text { an apartment building }\end{array}$ \\
\hline 43 & Villa Castelli & $\mathrm{IT}$ & Res / rural & $x$ & - & - \\
\hline 44 & Ansitz Kofler & $\mathrm{IT}$ & Res / urban & $x$ & - & - \\
\hline 45 & $\begin{array}{l}\text { Basilica di Santa Maria di } \\
\text { Collemaggio }\end{array}$ & $\mathrm{IT}$ & Religious & $x$ & - & - \\
\hline 46 & House Pernter & $\mathrm{IT}$ & Res / rural & $x$ & - & $\begin{array}{l}\text { Transformation into two } \\
\text { separate apartments }\end{array}$ \\
\hline 47 & Kohlerhaus & IT & Res / urban & $x$ & - & $\begin{array}{l}\text { Transformation into ten } \\
\text { apartments }\end{array}$ \\
\hline 48 & Ruckenzaunerhof & $\mathrm{IT}$ & Res / rural & $x$ & $\begin{array}{l}\text { Extension in the } \\
\text { west for additional } \\
\text { living space }\end{array}$ & - \\
\hline 49 & Aussergrubhof & $\mathrm{IT}$ & Res / rural & $x$ & $\begin{array}{l}\text { Heating system in } \\
\text { an external } \\
\text { underground room }\end{array}$ & $\begin{array}{l}\text { In addition to the residential } \\
\text { use two holiday apartments } \\
\text { were integrated }\end{array}$ \\
\hline 50 & Oberbergerhof & $\mathrm{IT}$ & Res / rural & $x$ & - & - \\
\hline 51 & Platzbonhof & $\mathrm{IT}$ & Res / rural & $x$ & - & $\begin{array}{l}\text { In addition to agricultural } \\
\text { and residential use two } \\
\text { holiday units were integrated }\end{array}$ \\
\hline 52 & Mairhof & $\mathrm{IT}$ & Res / rural & $x$ & $\begin{array}{l}\text { Extension } \\
\text { residential }\end{array}$ & - \\
\hline 53 & Obergasserhof & $\mathrm{IT}$ & Res / rural & $x$ & $\begin{array}{l}\text { Extension of two } \\
\text { stairways outside } \\
\text { of the house. } \\
\text { Heating system } \\
\text { extended }\end{array}$ & $\begin{array}{l}\text { A holiday apartment was } \\
\text { integrated into the attic }\end{array}$ \\
\hline 54 & Rebecco Farm & $\mathrm{IT}$ & B\&B - Farm & $x$ & - & $\begin{array}{l}\text { Transformation from } \\
\text { abandoned farm to } B \& B \text { and } \\
\text { educational use }\end{array}$ \\
\hline 55 & Villa Capodivacca & IT & Res / urban & $x$ & - & $\begin{array}{l}\text { Transformation from a } \\
\text { single-family house to three } \\
\text { apartments (one per floor) }\end{array}$ \\
\hline 56 & House Moroder & $\mathrm{IT}$ & Res / urban & $x$ & - & - \\
\hline 57 & Huberhof & IT & Res / rural & $x$ & - & $\begin{array}{l}\text { Transformation into two } \\
\text { apartments }\end{array}$ \\
\hline 58 & Notarjeva vila & SL & Res / urban & $x$ & - & $\begin{array}{l}\text { Transformation from four to } \\
\text { six apartment building }\end{array}$ \\
\hline 59 & Rožna ulica 15, Idrija & SL & Res / urban & $x$ & $\begin{array}{l}\text { Extension in the } \\
\text { attic for extra living } \\
\text { area }\end{array}$ & - \\
\hline 60 & Hiša trentarskih vodnikov & SL & Multipurpose & $x$ & $\begin{array}{l}\text { Extension in the } \\
\text { attic }\end{array}$ & $\begin{array}{l}\text { Transformation into a multi- } \\
\text { purpose building }\end{array}$ \\
\hline 61 & Idrija mercury smelting plant & SL & Educational & $x$ & $\begin{array}{l}\text { Extension for } \\
\text { reception of } \\
\text { visitors }\end{array}$ & $\begin{array}{l}\text { The former industrial plant } \\
\text { became a museum }\end{array}$ \\
\hline 62 & Mercado del Val, Valladolid & SP & Retail & $x$ & $\begin{array}{l}\text { Supermarket } \\
\text { added in the } \\
\text { basement of the } \\
\text { building }\end{array}$ & - \\
\hline 63 & Correria 119 & SP & Res / urban & $x$ & - & - \\
\hline 64 & Ahmet Aga Mansion & TK & Offices & $x$ & - & $\begin{array}{l}\text { Transformation to an office } \\
\text { building }\end{array}$ \\
\hline 65 & Nwcip Pasa Library & TK & Library & $x$ & - & - \\
\hline 66 & Downie's Cottage & UK & Res / rural & $x$ & - & $\begin{array}{l}\text { Transformation from } \\
\text { abandoned to residential } \\
\text { (holiday) use }\end{array}$ \\
\hline
\end{tabular}




\begin{tabular}{|l|l|l|l|l|l|l|}
\hline 67 & Holyrood Park Lodge & UK & Retail & X & - & $\begin{array}{l}\text { Transformation from a } \\
\text { residential building to a } \\
\text { building with retail use }\end{array}$ \\
\hline 68 & Annat Road & UK & Res / urban & X & - & - \\
\hline 69 & Aspinall Courthouse & USA & Offices & X & - & - \\
\hline
\end{tabular}




\section{Planning process}

\subsection{Heritage value assessment}

Most, but not all, assessed buildings are officially protected for their heritage values (see above section 2.3). The "Heritage significance" heading is divided in two sections: "Elements worthy of preservation" and "Heritage value assessment". In most cases of case study descriptions, these two sections intermingle.

In most projects, there is information about which elements are worthy of preservation. Given the limited text length, this information can only give examples or be very general, for example that the façade is protected. In several cases, this section describes the architecture of the building or the building type.

A few projects give clues on how the heritage value assessments have been carried out. In some cases, it is plainly stated that the building is protected according to the national heritage legislation, and no more information is given. Interestingly, in a number of cases it is mentioned that the building owner has complemented the heritage assessment and added elements worthy of preservation: "The protection decret mentions the neoclassical facade and a Madonna relief in marble above the flat arched main entrance. The owner himself added as elements worthy of preservation the old jasmine, the wine grape in the north corner going up to the terrasse and the tile stove in the living room" (Ansitz Kofler).

Also, when the building is lacking official recognition, the building owner has had aspirations to preserve the "character" of the building: "Das Gebäude steht nicht unter Denkmalschutz, dennoch haben die Besitzer bei den Sanierungsarbeiten darauf geachtet, dass der ursprüngliche Charakter des Hofes erhalten wird." (Oberbergerhof).

As mentioned, a few projects give clues on how the heritage assessment has been carried out and the role in the planning process. One of these is Villa Castelli, where it is evident that initially there was a lack of detailed heritage value assessment and that this hampered the process: "...there was no detailed assessment prior to the retrofit planning /.../ The only document the building owner had, was a vague description that all vertical structures and ceilings had to be maintained and that no intervention from outside was possible. /.../ the planning team was proposing a solution to the heritage office and in case they declined, the planning team proposed a new solution (often several times) until it was approved." (Villa Castelli).

In contrast, as far as project reporting a more successful collaboration with the heritage authorities is Rainhof, stressing: "open dialogue and the mutual support between building owner, architect and heritage office". (Rainhof). Also, Maison Rubens, a non-listed Belgian building, has a more detailed descriptions of how the heritage value assessment was carried out, using a number of criteria for different interests. In the case of Ryesgade, a quantitative system based on the SAVE-methodology was used. In one case, some correspondence with the planning authorities about the project has been attached. This gives invaluable information about the unfolding of the planning process (Residential and commercial building Feldbergstrasse - Basel).

An interesting outlier is Correria 119, where a participatory process was used the planning process: "The renovation process and the heritage value assessment were defined through a participatory process in two workshops where relevant stakeholders participate. In the first workshop, it was proposed a multicriteria exercise where the stakeholders decided which of the criteria (energy efficiency, heritage impact, economic feasibility...) were more important. The impact of the solutions on cultural values was considered a priority and these cultural values where identified. In the second, the stakeholders were involved in a co-creation process to choose solutions that would respect the identified heritage values of the building. A shortlist of measures was discussed and the impact evaluated to select the final solutions. (Correria 119)

Given the limited information that is available about the heritage assessments, it is not possible to perform an indepth analysis of how heritage assessments have been carried out, or what role they have played in the planning process. 


\subsection{Reason for refurbishment}

A majority of the buildings have been in a poor state and have been refurbished for residential use, with improved functionality (including thermal comfort) and improved energy performance as the main goals.

The Solar silo and Osramhuset are former industrial buildings that have been transformed into office spaces. Two buildings (Mariahilferstrasse and Basilica di Santa Maria di Collemaggio) were rebuilt after partial demolition due to a gas explosion and an earthquake, respectively. Several buildings are farm buildings, either dwellings or barns that have lost their previous function. These have been refurbished to modern use, mainly as dwellings (with examples of both permanent use and as holiday homes with high living standard). See also chapter 2.5. In some of the buildings that are occupied by the building owners themselves, it is emphasized that the aim was to create an atmosphere of well-being and comfort, while at the same time preserving the appearance or character of the building.

All projects have had an ambitious target for energy performance post refurbishment, but only a few have had an explicit quantitative target. Most of the projects have instead had an "as low as possible" approach. A few projects have reported an explicit aim to reduce carbon emissions over the whole life cycle, in addition to a lowered energy use. A few projects have had unusually ambitious targets for energy performance, often involving microgeneration (e.g. St. Franziskus Church, Kindergarten Chur). Musikschule Velden participated in a funding program to become a "model renovation", which was supportive of achieving sustainability targets.

Other mentioned reasons are to support local craftspeople, to use bio-based materials, to be a prototype for other buildings in the area. One project (Necip Pasa Library) mentions a good indoor climate for preservation as the main aim for refurbishment. Also, adaptation to climate change is mentioned in one project (Annat Road)

One has to keep in mind that the reasons for refurbishment described in the database is what project owners have communicated themselves post intervention. The true reasons might therefore differ from those stated.

\subsection{Use of specific tools}

Within the case studies a considerable number of different tools were used e.g. for detailed energy calculation, building physics or dynamic simulation. Some projects have used the same tools, indicated with bars (I).

- PHPP ॥

- Delphin II

- Standard calculation tools for regional or national energy certification (Austrian, South Tyrol)

- Econ calc

- Ham4D_VIE

- Hygrothermal monitoring II

- Flow meters

- EPB-software v8.0.4

- $\mathrm{LCA}$

- be15

- Wufi II

- Energy plus II

- Ansys fluent

- Klimahaus IIII

- TRNSYS

- SIA 380/1:2009

- Thermal imaging

- Therm

Regarding the use of systematic process, this is a yes/no question in the database. Therefore, it is not possible to draw conclusions. Three projects have answered yes (Obergasserhof, Holyrood lodge and Alken) but there is no information about which process. Probably, more projects have followed some sort of systematic process.

\subsection{Interdisciplinary planning team}

It is not possible to draw any conclusions from the information given in this section. In many projects (esp. the larger ones) there are several different competencies involved, but it is not known to what extent they have been involved and in what phase of the project. 


\section{Types of measures}

\subsection{Energy efficiency measures}

In the following, the various refurbishment measures implemented in the 69 best practice cases are described in a summarised form.

Table 8 shows an overview on which measures were implemented in each case study.

Table 8: Overview on measures implemented in the case studies

\begin{tabular}{|c|c|c|c|c|c|c|c|c|c|c|c|c|c|c|}
\hline \multirow[b]{2}{*}{ 은 } & \multirow[b]{2}{*}{ 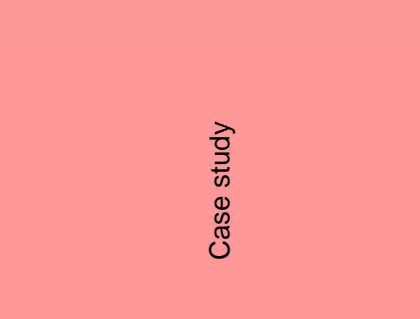 } & \multirow[b]{2}{*}{ 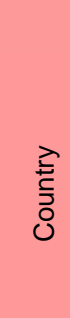 } & \multicolumn{3}{|c|}{$\mathrm{EE}$} & \multicolumn{4}{|c|}{ HVAC } & \multicolumn{5}{|c|}{ REN } \\
\hline & & & $\frac{\infty}{\bar{N}}$ & 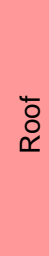 & 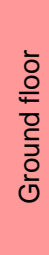 & 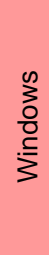 & 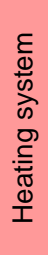 & 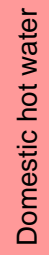 & 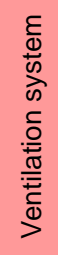 & 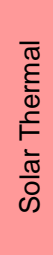 & $\begin{array}{l}\frac{0}{\pi} \\
\frac{T}{0} \\
\frac{0}{0} \\
\frac{0}{0} \\
\frac{C}{0}\end{array}$ & $\begin{array}{l}\mathscr{N} \\
\mathbb{0} \\
\text { Eั } \\
\text { 으 }\end{array}$ & 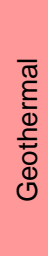 & $\begin{array}{l}\text { ठ্র } \\
\frac{0}{0} \\
\frac{1}{0} \\
\frac{0}{3} \\
\sum_{3}\end{array}$ \\
\hline 1 & Klostergebäude Kaiserstrasse & AT & --- & $x$ & --- & $x$ & $x$ & $x$ & $x$ & --- & --- & --- & --- & --- \\
\hline 2 & Farm house Trins & AT & $x$ & $x$ & -- & $x$ & $x$ & $x$ & $x$ & --- & --- & $x$ & $x$ & --- \\
\hline 3 & Hof 6, Schwarzenberg & AT & $x$ & $x$ & $x$ & $x$ & $x$ & $x$ & --- & $x$ & -- & $x$ & -- & -- \\
\hline 4 & Kelchalm & AT & $x$ & $x$ & --- & $x$ & $x$ & $x$ & $x$ & $x$ & --- & $x$ & --- & --- \\
\hline 5 & Mariahilferstrasse 182 & AT & $x$ & $x$ & --- & --- & $x$ & --- & $x$ & $x$ & --- & --- & --- & --- \\
\hline 6 & House Maurer, Wolfurt & AT & $x$ & $x$ & $x$ & $x$ & $x$ & --- & --- & $x$ & --- & --- & --- & --- \\
\hline 7 & House Breuer, Tschagguns & AT & $x$ & $x$ & $x$ & $x$ & $x$ & $x$ & $x$ & $x$ & $x$ & $x$ & --- & --- \\
\hline 8 & Music school in Velden & AT & $x$ & $x$ & $x$ & $x$ & $x$ & --- & $x$ & --- & --- & --- & --- & --- \\
\hline 9 & Hof Neuhäusl & AT & $x$ & $x$ & $x$ & $x$ & $x$ & $x$ & $x$ & --- & --- & --- & --- & --- \\
\hline 10 & Community Hall & AT & $x$ & $x$ & $x$ & $x$ & $x$ & $x$ & $x$ & --- & --- & --- & --- & --- \\
\hline 11 & Freihof Sulz & AT & $x$ & --- & --- & $x$ & $x$ & $x$ & $x$ & $x$ & --- & $x$ & --- & --- \\
\hline 12 & Oeconomy building & AT & $x$ & $x$ & $x$ & $x$ & $x$ & $x$ & $x$ & --- & --- & --- & --- & --- \\
\hline 13 & Giatla Haus & AT & $x$ & $x$ & $x$ & $x$ & $x$ & $x$ & $x$ & --- & --- & $x$ & --- & --- \\
\hline 14 & Rhine Valley House Irgang & AT & $x$ & $x$ & $x$ & $x$ & $x$ & $x$ & --- & $x$ & --- & $x$ & --- & --- \\
\hline 15 & Baur Residence, Lustenau & AT & $x$ & $x$ & --- & $x$ & $x$ & $x$ & --- & $x$ & --- & $x$ & --- & --- \\
\hline 16 & Kasperhof & AT & $x$ & $x$ & $x$ & $x$ & $x$ & $x$ & --- & --- & --- & --- & --- & --- \\
\hline 17 & Maison Rubens & $\mathrm{BE}$ & $x$ & $x$ & $x$ & $x$ & $x$ & $x$ & $x$ & $x$ & --- & $x$ & --- & --- \\
\hline 18 & Half-timberframed house & $\mathrm{BE}$ & $x$ & $x$ & $x$ & $x$ & $x$ & $x$ & $x$ & --- & --- & --- & --- & --- \\
\hline 19 & Doragno Castle, Rovio & $\mathrm{CH}$ & $x$ & $x$ & $x$ & $x$ & $x$ & $x$ & $x$ & $x$ & $x$ & --- & --- & --- \\
\hline 20 & Solar silo & $\mathrm{CH}$ & $x$ & $x$ & --- & $x$ & $x$ & --- & $x$ & --- & $x$ & --- & --- & --- \\
\hline 21 & Wohn- und Geschäftshaus & $\mathrm{CH}$ & $x$ & $x$ & $x$ & $x$ & $x$ & $x$ & $x$ & $x$ & $x$ & --- & -- & -- \\
\hline 22 & Mehrfamilienhaus & $\mathrm{CH}$ & $x$ & $x$ & $x$ & $x$ & $x$ & $x$ & $x$ & $x$ & --- & $x$ & --- & --- \\
\hline 23 & St. Franziskus Church & $\mathrm{CH}$ & --- & $x$ & --- & $x$ & $x$ & $x$ & --- & $x$ & $x$ & --- & $x$ & --- \\
\hline 24 & Kindergarten and apartments & $\mathrm{CH}$ & $x$ & $x$ & $x$ & $x$ & $x$ & $x$ & $x$ & $x$ & $x$ & $x$ & --- & --- \\
\hline 25 & Single family home & $\mathrm{CH}$ & $x$ & $x$ & $x$ & $x$ & $x$ & $x$ & $x$ & $x$ & $x$ & $x$ & $x$ & --- \\
\hline 26 & Single Family House - Gstaad & $\mathrm{CH}$ & $x$ & $x$ & $x$ & $x$ & $x$ & $x$ & --- & --- & $x$ & --- & --- & --- \\
\hline 27 & Glaserhaus in Affoltern & $\mathrm{CH}$ & $x$ & $x$ & $x$ & $x$ & $x$ & $x$ & $x$ & --- & $x$ & --- & $x$ & --- \\
\hline 28 & PalaCinema Locarno & $\mathrm{CH}$ & $x$ & $x$ & $x$ & $x$ & $x$ & $x$ & $x$ & --- & $x$ & --- & $x$ & --- \\
\hline 29 & Casa Rossa Chemnitz & $\mathrm{DE}$ & $x$ & $x$ & --- & $x$ & $x$ & $x$ & $x$ & $x$ & --- & --- & --- & --- \\
\hline 30 & Rathaus Bergrheinfeld & $\mathrm{DE}$ & $x$ & $x$ & $x$ & $x$ & $x$ & --- & $x$ & --- & --- & --- & --- & --- \\
\hline 31 & Farmhouse Straub & $\mathrm{DE}$ & $x$ & $x$ & $x$ & $x$ & $x$ & $x$ & --- & $x$ & --- & --- & --- & --- \\
\hline 32 & Early work Sep Ruf & $\mathrm{DE}$ & $x$ & $x$ & $x$ & $x$ & $x$ & $x$ & --- & --- & --- & $x$ & --- & --- \\
\hline
\end{tabular}




\begin{tabular}{|c|c|c|c|c|c|c|c|c|c|c|c|c|c|c|}
\hline 33 & Ackerbürgerhäuschen & $\mathrm{DE}$ & $x$ & $x$ & $x$ & $x$ & $x$ & $x$ & $x$ & --- & --- & --- & --- & --- \\
\hline 34 & Ritterhof & $\mathrm{DE}$ & $x$ & $x$ & $x$ & $x$ & $x$ & $x$ & $x$ & $x$ & $x$ & $x$ & --- & --- \\
\hline 35 & Rathaus Burgkunstadt & $\mathrm{DE}$ & $x$ & $x$ & $x$ & $x$ & $x$ & $x$ & $x$ & --- & --- & --- & --- & --- \\
\hline 36 & Osramhuset & DK & $x$ & --- & --- & $x$ & $x$ & $x$ & $x$ & --- & --- & --- & --- & --- \\
\hline 37 & Klitgaarden & DK & $x$ & $x$ & $x$ & $x$ & $x$ & --- & $x$ & --- & --- & --- & --- & --- \\
\hline 38 & RYESGADE 30 & DK & $x$ & $x$ & --- & $x$ & $x$ & --- & $x$ & --- & $x$ & --- & --- & --- \\
\hline 39 & Timber-framed house & FR & $x$ & $x$ & $x$ & $x$ & $x$ & $x$ & $x$ & --- & --- & $x$ & --- & --- \\
\hline 40 & Elementary School in & $\mathrm{FR}$ & $x$ & $x$ & $x$ & $x$ & $x$ & --- & $x$ & --- & --- & --- & --- & --- \\
\hline 41 & Timber-framed barn & FR & $x$ & $x$ & $x$ & $x$ & $x$ & --- & $x$ & --- & --- & --- & --- & --- \\
\hline 42 & Rainhof & IT & $x$ & $x$ & $x$ & $x$ & $x$ & $x$ & $x$ & $x$ & $x$ & $x$ & $x$ & $x$ \\
\hline 43 & Villa Castelli & $\mathrm{IT}$ & $x$ & $x$ & $x$ & $x$ & $x$ & $x$ & $x$ & --- & --- & --- & $x$ & $x$ \\
\hline 44 & Ansitz Kofler & IT & $x$ & $x$ & $x$ & $x$ & $x$ & $x$ & $x$ & --- & --- & $x$ & $x$ & --- \\
\hline 45 & Basilica di Santa Maria di C. & IT & --- & --- & --- & $x$ & $x$ & --- & --- & --- & --- & --- & $x$ & --- \\
\hline 46 & House Pernter & $\mathrm{IT}$ & $x$ & --- & $x$ & $x$ & $x$ & $x$ & $x$ & $x$ & --- & $x$ & --- & --- \\
\hline 47 & Kohlerhaus & $\mathrm{IT}$ & $x$ & $x$ & $x$ & $x$ & $x$ & $x$ & $x$ & --- & --- & --- & --- & --- \\
\hline 48 & Ruckenzaunerhof & IT & $x$ & $x$ & $x$ & $x$ & $x$ & $x$ & --- & --- & --- & --- & --- & --- \\
\hline 49 & Aussergrubhof & $\mathrm{IT}$ & $x$ & $x$ & $x$ & $x$ & $x$ & $x$ & --- & --- & --- & $x$ & --- & -- \\
\hline 50 & Oberbergerhof & IT & $x$ & $x$ & $x$ & $x$ & $x$ & $x$ & --- & --- & --- & $x$ & --- & --- \\
\hline 51 & Platzbonhof & $\mathrm{IT}$ & $x$ & $x$ & $x$ & $x$ & $x$ & $x$ & --- & --- & --- & $x$ & --- & --- \\
\hline 52 & Mairhof & $\mathrm{IT}$ & $x$ & $x$ & $x$ & $x$ & $x$ & $x$ & $x$ & $x$ & --- & $x$ & --- & --- \\
\hline 53 & Obergasserhof & IT & --- & $x$ & $x$ & $x$ & $x$ & $x$ & -- & --- & --- & $x$ & --- & --- \\
\hline 54 & Rebecco Farm & IT & $x$ & $x$ & --- & $x$ & $x$ & --- & --- & --- & --- & $x$ & --- & --- \\
\hline 55 & Villa Capodivacca & $\mathrm{IT}$ & $x$ & $x$ & --- & $x$ & $x$ & $x$ & $x$ & --- & --- & --- & --- & --- \\
\hline 56 & House Moroder & $\mathrm{IT}$ & $x$ & --- & $x$ & $x$ & $x$ & -- & $x$ & --- & --- & --- & --- & --- \\
\hline 57 & Huberhof & $\mathrm{IT}$ & --- & --- & --- & --- & --- & --- & --- & --- & --- & --- & --- & --- \\
\hline 58 & Notarjeva vila & $\mathrm{SL}$ & $x$ & $x$ & $x$ & $x$ & $x$ & $x$ & --- & --- & --- & $x$ & --- & --- \\
\hline 59 & Rožna ulica 15, Idrija & $\mathrm{SL}$ & $x$ & $x$ & $x$ & $x$ & $x$ & $x$ & --- & --- & --- & --- & --- & --- \\
\hline 60 & Hiša trentarskih vodnikov & $\mathrm{SL}$ & $x$ & $x$ & --- & $x$ & $x$ & $x$ & --- & --- & --- & $x$ & --- & --- \\
\hline 61 & Idrija mercury smelting plant & $\mathrm{SL}$ & $x$ & $x$ & --- & $x$ & $x$ & $x$ & --- & --- & --- & $x$ & --- & --- \\
\hline 62 & Mercado del Val, Valladolid & $\mathrm{SP}$ & --- & $x$ & $x$ & $x$ & $x$ & $x$ & $x$ & --- & --- & --- & $x$ & --- \\
\hline 63 & Correria 119 & $\mathrm{SP}$ & $x$ & $x$ & --- & $x$ & $x$ & $x$ & --- & --- & --- & --- & --- & --- \\
\hline 64 & Ahmet Aga Mansion & TK & --- & $x$ & $x$ & $x$ & $x$ & --- & --- & --- & --- & --- & --- & --- \\
\hline 65 & Nwcip Pasa Library & TK & $x$ & --- & $x$ & $x$ & $x$ & --- & $x$ & --- & --- & --- & --- & --- \\
\hline 66 & Downie's Cottage & UK & $x$ & $x$ & $x$ & $x$ & $x$ & $x$ & --- & --- & --- & --- & $x$ & --- \\
\hline 67 & Hollyrood Park Lodge & UK & $x$ & $x$ & $x$ & $x$ & $x$ & --- & $x$ & --- & --- & --- & --- & --- \\
\hline 68 & Annat Road & UK & $x$ & $x$ & $x$ & $x$ & $x$ & --- & $x$ & --- & --- & --- & --- & --- \\
\hline 69 & Aspinall Courthouse & USA & $x$ & $x$ & --- & $x$ & $x$ & --- & $x$ & --- & $x$ & --- & $x$ & --- \\
\hline
\end{tabular}




\subsubsection{Walls}

From the 69 best practice examples, 6 examples did not carry out thermal measures on the walls. As some buildings have several documented wall solutions a total of 67 different wall concepts are described. A large part, namely 41 of these thermal wall improvements were achieved by internal insulation. In 22 solutions the thermal improvement was realized with external insulation and in one case a cavity insulation was implemented.

\subsubsection{Overview of wall-solutions}

Table 9 shows an overview, which kinds of insulations were used for new wall solutions, the U-values before and after the intervention and what kind of wall was there initially.

Table 9: Overview of wall-solutions

\begin{tabular}{|c|c|c|c|c|c|c|}
\hline \multirow[t]{2}{*}{ No } & \multirow[t]{2}{*}{$\begin{array}{l}\text { 홍 } \\
\text { 苟 } \\
0 \\
0 \\
0 \\
0 \\
0\end{array}$} & \multirow[t]{2}{*}{ 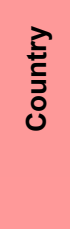 } & \multirow[t]{2}{*}{ 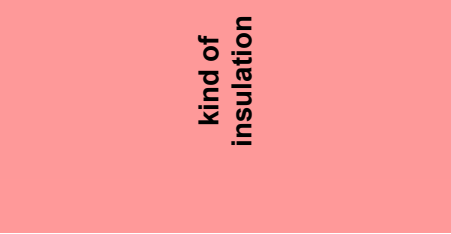 } & \multicolumn{2}{|c|}{ 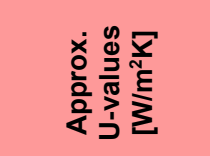 } & \multirow[t]{2}{*}{ 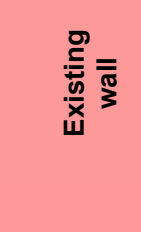 } \\
\hline & & & & before & after & \\
\hline 1 & Klostergebäude Kaiserstrasse & AT & internal insulation - mineral wool & 0.92 & 0.44 & $\begin{array}{l}\text { Solid brick } \\
\text { wall }\end{array}$ \\
\hline 2 & Farm house Trins & AT & $\begin{array}{l}\text { external reversible system - } \\
\text { cellulose }\end{array}$ & 2.13 & 0.12 & $\begin{array}{l}\text { Stone } \\
\text { masonry }\end{array}$ \\
\hline 3 & $\begin{array}{l}\text { Hof 6, Schwarzenberg, } \\
\text { Voralberg }\end{array}$ & AT & internal insulation - wood fibre & 1.20 & 0.60 & Log wall \\
\hline 4 & $\begin{array}{l}\text { Kelchalm - Bochumer alpine } \\
\text { hut }\end{array}$ & AT & $\begin{array}{l}\text { external insulation with hemp } \\
\text { external insulation with cellulose }\end{array}$ & $\begin{array}{l}1.56 \\
0.34\end{array}$ & $\begin{array}{l}0.20 \\
0.19\end{array}$ & $\begin{array}{l}\text { Stone } \\
\text { masonry } \\
\text { Log wall }\end{array}$ \\
\hline 5 & Mariahilferstrasse 182 & AT & $\begin{array}{l}\text { external insulation with aerogel - } \\
\text { plaster }\end{array}$ & 1.13 & 0.30 & $\begin{array}{l}\text { Solid brick } \\
\text { wall }\end{array}$ \\
\hline 6 & House Maurer, Wolfurt & AT & $\begin{array}{l}\text { external / internal insulation with } \\
\text { wood fibre }\end{array}$ & 0.62 & 0.20 & Log wall \\
\hline 7 & House Breuer, Tschagguns & AT & $\begin{array}{l}\text { internal insulation between } \\
\text { wooden construction }\end{array}$ & 3.00 & 0.17 & $\begin{array}{l}\text { Wooden } \\
\text { formwork }\end{array}$ \\
\hline 8 & Music school in Velden & AT & internal insulation - calcium silicate & 1.18 & 0.38 & $\begin{array}{l}\text { Solid brick } \\
\text { wall }\end{array}$ \\
\hline 9 & Hof Neuhäusl & AT & internal insulation - cellulose & 0.86 & 0.15 & Log wall \\
\hline 10 & $\begin{array}{l}\text { Community Hall } \\
\text { Zwischenwasser }\end{array}$ & AT & internal insulation - calcium silicate & 0.79 & 0.31 & $\begin{array}{l}\text { Solid brick } \\
\text { wall }\end{array}$ \\
\hline 11 & Freihof Sulz & AT & internal insulation - wood fibre & 1.26 & 0.45 & $\begin{array}{l}\text { Stone } \\
\text { masonry }\end{array}$ \\
\hline 12 & $\begin{array}{l}\text { Oeconomy building Josef } \\
\text { Weiss }\end{array}$ & AT & $\begin{array}{l}\text { internal insulation between } \\
\text { wooden construction }\end{array}$ & 3.00 & 0.23 & $\begin{array}{l}\text { Wooden } \\
\text { formwork }\end{array}$ \\
\hline 13 & Giatla Haus & AT & internal insulation - sheep wool & 0.85 & 0.35 & Log wall \\
\hline 14 & Rhine Valley House Irgang & AT & external insulation - EPS & $\begin{array}{r}2 \\
0.53\end{array}$ & $\begin{array}{l}0.21 \\
0.17\end{array}$ & $\begin{array}{l}\text { Stone } \\
\text { masonry } \\
\text { Log wall }\end{array}$ \\
\hline 15 & Baur Residence, Lustenau & AT & $\begin{array}{l}\text { external insulation - mineral wool / } \\
\text { shingles }\end{array}$ & 0.51 & 0.15 & Log wall \\
\hline 16 & Kasperhof & AT & $\begin{array}{l}\text { internal insulation - mineral foam } \\
\text { panels }\end{array}$ & 2.14 & 0.34 & $\begin{array}{l}\text { Stone } \\
\text { masonry }\end{array}$ \\
\hline 17 & Maison Rubens & $\mathrm{BE}$ & internal insulation - wood fibre & - & 0.41 & $\begin{array}{l}\text { Solid brick } \\
\text { wall }\end{array}$ \\
\hline 18 & $\begin{array}{l}\text { Half-timberframed house in } \\
\text { Alken }\end{array}$ & $\mathrm{BE}$ & $\begin{array}{l}\text { insulation between framework - } \\
\text { wood fiber / mineral wool }\end{array}$ & 1.93 & 0.29 & $\begin{array}{l}\text { wooden } \\
\text { framework } \\
\text { (loam, } \\
\text { bricks) }\end{array}$ \\
\hline 19 & Doragno Castle, Rovio & $\mathrm{CH}$ & internal insulation - mineral wool & 0.90 & 0.24 & $\begin{array}{l}\text { Stone } \\
\text { masonry }\end{array}$ \\
\hline 20 & Solar silo & $\mathrm{CH}$ & $\begin{array}{l}\text { external insulation - mineral wool / } \\
\text { cladding }\end{array}$ & 2.00 & 0.16 & Concrete \\
\hline 21 & $\begin{array}{l}\text { Wohn- und Geschäftshaus } \\
\text { Feldbergstrasse }\end{array}$ & $\mathrm{CH}$ & external insulation - mineral wool & 1.40 & 0.16 & $\begin{array}{l}\text { Solid brick } \\
\text { wall }\end{array}$ \\
\hline 22 & $\begin{array}{l}\text { Mehrfamilienhaus } \\
\text { Magnusstrasse }\end{array}$ & $\mathrm{CH}$ & external insulation - mineral wool & 0.80 & 0.12 & $\begin{array}{l}\text { Solid brick } \\
\text { wall }\end{array}$ \\
\hline 23 & $\begin{array}{l}\text { St. Franziskus Church } \\
\text { Ebmatingen }\end{array}$ & $\mathrm{CH}$ & - & - & - & - \\
\hline
\end{tabular}




\begin{tabular}{|c|c|c|c|c|c|c|}
\hline 24 & $\begin{array}{l}\text { Kindergarten and apartments } \\
\text { (PEB) Chur }\end{array}$ & $\mathrm{CH}$ & $\begin{array}{l}\text { external insulation - multipor } \\
\text { (mineral foam) }\end{array}$ & 2.50 & 0.14 & $\begin{array}{l}\text { Stone } \\
\text { masonry }\end{array}$ \\
\hline 25 & $\begin{array}{l}\text { Single family home } \\
\text { Luisenstrasse }\end{array}$ & $\mathrm{CH}$ & cavity insulation - Isofloc & 0.68 & 0.44 & $\begin{array}{l}\text { Double shell } \\
\text { masonry } \\
\text { wall }\end{array}$ \\
\hline 26 & Single Family House - Gstaad & $\mathrm{CH}$ & $\begin{array}{l}\text { external insulation - stone wool } \\
\text { internal insulation - not described }\end{array}$ & $\begin{array}{l}- \\
-\end{array}$ & $\begin{array}{l}0.2 \\
0.2\end{array}$ & $\begin{array}{l}\text { Log wall } \\
\text { Log wall }\end{array}$ \\
\hline 27 & Glaserhaus in Affoltern & $\mathrm{CH}$ & $\begin{array}{l}\text { internal insulation between } \\
\text { wooden construction }\end{array}$ & 3.00 & 0.16 & $\begin{array}{l}\text { Wooden } \\
\text { formwork }\end{array}$ \\
\hline 28 & PalaCinema Locarno & $\mathrm{CH}$ & internal insulation - mineral wool & - & 0.15 & $\begin{array}{l}\text { Stone } \\
\text { masonry }\end{array}$ \\
\hline 29 & Casa Rossa Chemnitz & $\mathrm{DE}$ & external insulation - mineral wool & 0.85 & 0.20 & $\begin{array}{l}\text { Solid brick } \\
\text { wall }\end{array}$ \\
\hline 30 & Rathaus Bergrheinfeld & $\mathrm{DE}$ & $\begin{array}{l}\text { internal insulation - insulating } \\
\text { plaster }\end{array}$ & $\begin{array}{l}1.16 \\
1.11\end{array}$ & $\begin{array}{l}0.68 \\
0.66\end{array}$ & $\begin{array}{l}\text { Wooden } \\
\text { framework } \\
\text { Solid brick } \\
\text { wall }\end{array}$ \\
\hline 31 & Farmhouse Straub & $\mathrm{DE}$ & $\begin{array}{l}\text { external insulation - blow in } \\
\text { cellulose } \\
\text { internal insulation - wood fibre / } \\
\text { clayrender } \\
\text { internal insulation - cellulose / } \\
\text { wood fibre }\end{array}$ & $\begin{array}{l}- \\
-\end{array}$ & $\begin{array}{l}0.13 \\
0.16 \\
0.11\end{array}$ & $\begin{array}{l}\text { Log wall } \\
\text { Log wall } \\
\text { wooden } \\
\text { formwork }\end{array}$ \\
\hline 32 & Early work Sep Ruf & $\mathrm{DE}$ & $\begin{array}{l}\text { external insulation - multipor } \\
\text { (mineral foam) }\end{array}$ & 1.42 & 0.49 & $\begin{array}{l}\text { Solid brick } \\
\text { wall }\end{array}$ \\
\hline 33 & Ackerbürgerhäuschen & $\mathrm{DE}$ & external insulaiton - mineral wool & 3.50 & $\begin{array}{r}0.13 \\
- \\
0.21\end{array}$ & $\begin{array}{l}\text { Solid brick } \\
\text { wall }\end{array}$ \\
\hline 34 & Ritterhof & $\mathrm{DE}$ & $\begin{array}{l}\text { internal insulation - flexibel wood } \\
\text { fibre } \\
\text { external insulation - blow in } \\
\text { cellulose }\end{array}$ & - & $\begin{array}{r}0.18 \\
0.115\end{array}$ & $\begin{array}{l}\text { Log wall } \\
\text { Log wall }\end{array}$ \\
\hline 35 & Rathaus Burgkunstadt & $\mathrm{DE}$ & $\begin{array}{l}\text { lightweight clay bricks between } \\
\text { framework / clay insulation board } \\
\text { inside }\end{array}$ & - & 0.77 & $\begin{array}{l}\text { wooden } \\
\text { framework }\end{array}$ \\
\hline 36 & $\begin{array}{l}\text { Osramhuset (The Osram } \\
\text { Building) }\end{array}$ & DK & $\begin{array}{l}\text { internal insulation covered by a } \\
\text { layer of glass }\end{array}$ & 2.24 & 0.13 & Concrete \\
\hline 37 & Klitgaarden & DK & internal insulation - no information & 1.40 & 0.30 & $\begin{array}{l}\text { Solid brick } \\
\text { wall }\end{array}$ \\
\hline 38 & RYESGADE 30 & DK & $\begin{array}{l}\text { internal insulation - Aerorock (mix } \\
\text { of rock wool and aerogel) }\end{array}$ & 1.40 & 0.40 & $\begin{array}{l}\text { Solid brick } \\
\text { wall }\end{array}$ \\
\hline 39 & $\begin{array}{l}\text { Timber-framed house in } \\
\text { Alsace }\end{array}$ & FR & $\begin{array}{l}\text { internal insulation - lime hemp } \\
\text { concrete }\end{array}$ & 1.75 & 0.39 & $\begin{array}{l}\text { wooden } \\
\text { framework }\end{array}$ \\
\hline 40 & $\begin{array}{l}\text { Elementary School in } \\
\text { Mulhouse }\end{array}$ & FR & internal insulation - mineral wool & 3.00 & 0.34 & $\begin{array}{l}\text { Stone } \\
\text { masonry }\end{array}$ \\
\hline 41 & $\begin{array}{l}\text { Timber-framed barn in the } \\
\text { north of France }\end{array}$ & FR & $\begin{array}{l}\text { internal insulation - hemp wool / } \\
\text { clay render }\end{array}$ & - & 0.4 & $\begin{array}{l}\text { wooden } \\
\text { framework }\end{array}$ \\
\hline 42 & Rainhof & IT & $\begin{array}{l}\text { internal insulation - insulating } \\
\text { plaster } \\
\text { internal insulation - wood } \\
\text { fibreboard behind wooden } \\
\text { cladding } \\
\text { internal insulation - wood fibre } \\
\text { coverd by OSB board + installation } \\
\text { layer }\end{array}$ & $\begin{array}{l}2.39 \\
1.33 \\
0.64\end{array}$ & $\begin{array}{l}0,87 \\
0,36 \\
0,24\end{array}$ & $\begin{array}{l}\text { Stone } \\
\text { masonry } \\
\text { Stone } \\
\text { masonry } \\
\text { Log wall }\end{array}$ \\
\hline 43 & Villa Castelli & IT & $\begin{array}{l}\text { internal insulation - perlite panel } \\
\text { internal insulation - aerogel }\end{array}$ & $\begin{array}{l}1.4- \\
1.33 \\
2.47\end{array}$ & $\begin{array}{l}0.19 \\
0.18\end{array}$ & $\begin{array}{l}\text { Solid brick } \\
\text { wall / Stone } \\
\text { masonry } \\
\text { Concrete }\end{array}$ \\
\hline 44 & Ansitz Kofler & IT & $\begin{array}{l}\text { internal insulation - mineral wool / } \\
\text { XPS (first half meter) } \\
\text { external insulation - mineral wool }\end{array}$ & $\begin{array}{l}2.10 \\
2.10\end{array}$ & $\begin{array}{l}0.17 \\
0.16\end{array}$ & $\begin{array}{l}\text { Stone } \\
\text { masonry } \\
\text { Stone } \\
\text { masonry }\end{array}$ \\
\hline 45 & $\begin{array}{l}\text { Basilica di Santa Maria di } \\
\text { Collemaggio }\end{array}$ & IT & - & - & - & - \\
\hline 46 & House Pernter & IT & external insulation - mineral wool & & & $\begin{array}{l}\text { Stone } \\
\text { masonry }\end{array}$ \\
\hline 47 & Kohlerhaus & IT & $\begin{array}{l}\text { external / internal insulation - reed } \\
\text { mat }\end{array}$ & 2.00 & 0.33 & $\begin{array}{l}\text { Stone } \\
\text { masonry }\end{array}$ \\
\hline 48 & Ruckenzaunerhof & IT & $\begin{array}{l}\text { internal insulation - insulating } \\
\text { plaster }\end{array}$ & 1.43 & 0.64 & $\begin{array}{l}\text { Stone } \\
\text { masonry }\end{array}$ \\
\hline 49 & Aussergrubhof & IT & internal insulation - hemp wool & 0.45 & 0.24 & Log wall \\
\hline
\end{tabular}




\begin{tabular}{|c|c|c|c|c|c|c|}
\hline 50 & Oberbergerhof & IT & house in house solution & - & 0.2 & $\begin{array}{l}\text { Stone } \\
\text { masonry }\end{array}$ \\
\hline 51 & Platzbonhof & IT & $\begin{array}{l}\text { external insulation - insulating } \\
\text { plaster }\end{array}$ & 2.22 & 0.44 & $\begin{array}{l}\text { Stone } \\
\text { masonry }\end{array}$ \\
\hline 52 & Mairhof & IT & $\begin{array}{l}\text { internal insulation - insulating } \\
\text { plaster }\end{array}$ & 1.85 & 0.48 & $\begin{array}{l}\text { Stone } \\
\text { masonry }\end{array}$ \\
\hline 53 & Obergasserhof & IT & - & - & - & - \\
\hline 54 & Rebecco Farm & IT & $\begin{array}{l}\text { internal insulation - hemp lime } \\
\text { bricks / lime - hemp plaster } \\
\text { internal insulation - reed panel / } \\
\text { hemp lime plaster }\end{array}$ & $\begin{array}{l}1.86 \\
1.90\end{array}$ & $\begin{array}{l}0.14 \\
0.54\end{array}$ & $\begin{array}{l}\text { Stone } \\
\text { masonry } \\
\text { Stone } \\
\text { masonry }\end{array}$ \\
\hline 55 & Villa Capodivacca & IT & internal insulation - calcium silicate & 1.72 & 0.39 & $\begin{array}{l}\text { Solid brick } \\
\text { wall }\end{array}$ \\
\hline 56 & House Moroder & IT & internal insulation - perlite panel & 2.5 & 0.38 & $\begin{array}{l}\text { Stone } \\
\text { masonry }\end{array}$ \\
\hline 57 & Huberhof & IT & $\begin{array}{l}\text { internal insulation - gaseous } \\
\text { concrete brick } \\
\text { internal insulation - wood fibre } \\
\text { behind panelling } \\
\text { internal insulation - wood fibre }\end{array}$ & $\begin{array}{l}0.71 \\
0.74 \\
0.93\end{array}$ & $\begin{array}{l}0.51 \\
0.42 \\
0.25\end{array}$ & $\begin{array}{l}\text { Stone } \\
\text { masonry } \\
\text { Stone } \\
\text { masonry } \\
\text { Wooden } \\
\text { framework }\end{array}$ \\
\hline 58 & Notarjeva vila & SL & external insulation & 0.86 & 0.23 & $\begin{array}{l}\text { Solid brick } \\
\text { wall }\end{array}$ \\
\hline 59 & Rožna ulica 15, Idrija & SL & external insulation & 1.51 & 0.21 & $\begin{array}{l}\text { Stone } \\
\text { masonry }\end{array}$ \\
\hline 60 & Hiša trentarskih vodnikov & SL & external insulation - XPS & 3.5 & 0.5 & $\begin{array}{l}\text { Stone } \\
\text { masonry }\end{array}$ \\
\hline 61 & Idrija mercury smelting plant & SL & - & - & - & $\begin{array}{l}\text { Concrete } \\
\text { filled with } \\
\text { bricks }\end{array}$ \\
\hline 62 & Mercado del Val, Valladolid & SP & New glazing facade & & & \\
\hline 63 & Correria 119 & $\mathrm{SP}$ & $\begin{array}{l}\text { internal insulation - recycled cotton } \\
\text { fibre insulation } \\
\text { external insulation - corkboard }\end{array}$ & $\begin{array}{l}0.82 \\
0.46\end{array}$ & $\begin{array}{l}0.43 \\
0.26\end{array}$ & $\begin{array}{l}\text { Stone } \\
\text { masonry } \\
\text { Solid brick } \\
\text { wall }\end{array}$ \\
\hline 64 & Ahmet Aga Mansion & TK & - & - & - & - \\
\hline 65 & Nwcip Pasa Library & TK & - & - & - & - \\
\hline 66 & Downie's Cottage & UK & $\begin{array}{l}\text { internal insulation - insulated lime } \\
\text { plaster }\end{array}$ & 1.76 & 1.23 & $\begin{array}{l}\text { Stone } \\
\text { masonry }\end{array}$ \\
\hline 67 & Hollyrood Park Lodge & UK & $\begin{array}{l}\text { internal insulation - blow in } \\
\text { cellulose behind existing plaster } \\
\text { lining }\end{array}$ & 1.3 & 0.67 & $\begin{array}{l}\text { Stone } \\
\text { masonry }\end{array}$ \\
\hline 68 & Annat Road & UK & $\begin{array}{l}\text { internal insulation - Water based } \\
\text { expanding foam injected into } \\
\text { cavity }\end{array}$ & 1.1 & 0.41 & $\begin{array}{l}\text { Stone } \\
\text { masonry }\end{array}$ \\
\hline 69 & Aspinall Courthouse & USA & $\begin{array}{l}\text { internal insulation - spray foam } \\
\text { insulation }\end{array}$ & 1.04 & 0.42 & $\begin{array}{l}\text { Stone } \\
\text { masonry }\end{array}$ \\
\hline
\end{tabular}

\subsubsection{Kind of insulation \& insulation system}

The majority of solutions are achieved by internal insulation. The main reason is that existing facades remain unchanged in their appearance. Internal insulation can increase the thermal resistance of the wall while preserving historic exterior plasters and paintings. For example, at the Ruckzaunerhof in Tarsch (Italy), the old plaster, which partly dates back to the 16th century, was worked out and the thermal resistance was increased by internal insulation. Due to the preservation of the historical facades, it is not always possible to create a surface that is resistant to driving rain. For that reason, it makes sense to use diffusion open capillary active internal insulation systems. It is not surprising that 28 out of the 41 solutions with internal insulation were executed with capillary-active materials or a diffusion-open structure with plasters. Due to the capillary activity and the diffusion open construction, moisture penetrating from outside can also dry out on the inside. Despite these systems, the issue of driving rain must be solved and any rising damp must be prevented or appropriately removed.

Insulating plasters were used to a large extent for the diffusible capillary-active internal insulation systems. These plasters were mostly applied in a thickness of $60-80 \mathrm{~mm}$ and achieve U-values between $0.39-1.23 \mathrm{~W} /\left(\mathrm{m}^{2} \cdot \mathrm{K}\right)$ depending on the external wall. A special feature is the Timber framed house in Alsace, where a $180 \mathrm{~mm}$ thick lime hemp plaster was applied and achieved a U-value of $0.39 \mathrm{~W} /\left(\mathrm{m}^{2} \cdot \mathrm{K}\right)$. In case of the use of board insulation materials, mostly wood fiber boards, aerogel boards, perlite boards and mineral wool boards were used. In the 
case of Villa Castelli, perlite boards with $200 \mathrm{~mm}$ as well as $80 \mathrm{~mm}$ aerogel boards were installed and achieved a U-value of $0.18-0.19 \mathrm{~W} /\left(\mathrm{m}^{2} \cdot \mathrm{K}\right)$. Cellulose fibers were used as blow-in insulation.

The second most frequent, namely in 12 cases out of 41 , internal insulation systems with vapour retardant layers were used. Especially for log walls, mostly OSB boards were used as vapour retarders. The OSB board is also used as an airtight level. In these cases, attention must be paid to the choice of the company of the OSB board, as there are big differences in the air-tightness of the boards. In the other cases, vapour retarders were made out of polyethylene foil. At the elementary school "Cour de Larraine" a hygrovariable vapour retardant layer was installed. The U-values achieved with the vapour retardant systems are in the range of $0.15-0.60 \mathrm{~W} /\left(\mathrm{m}^{2} \cdot \mathrm{K}\right)$.

In comparison to the diffusion-open capillary-active systems, the vapour retarding systems usually rely on insulation in the form of soft panels or blow in insulation. At the "Rainhof" and the "Hof 6, Schwarzenberg", wood fiber boards were used for the insulation of the log walls. Hemp fiber boards were attached to the wooden walls of the "Aussergrubhof" and mineral wool boards were used for most of the remaining examples.

Actually, there is only one example where a vapour-tight internal insulation system has been chosen. This is the best practice example Osramhuset in Denmark. There a layer of glass was applied to the inside of the building.

With regard to the choice of materials, there is a general trend towards ecological materials. EPS and XPS have almost never been used. However, the use of ecological materials such as hemp fiber, wood fiber and cellulose fiber is quite common. In the field of plasters, a trend towards lime and clay plasters is noticeable.

In 22 out of the 67 cases, the thermal improvement was achieved by external insulation. In almost all these cases mineral wool insulation was used for this purpose. In the example of Mariahilfstraße 182 an ecological hemp insulation was used. At the Kelchalm in Austria a blow in cellulose system was applied. In two cases an improvement was achieved by a thermal insulation plaster. One of these was aerogel plaster and the other lime insulation plaster from the company Röfix (CalceClima Thermo). Other materials used were mineral foam, reed mat and XPS.

A reversible solution was also implemented in one case study: at Farmhouse Trins in Austria, reversible wood fibre boards with cellulose cavity insulation were installed.

\subsubsection{Technical compatibility}

Especially for the renovation of old buildings, the requirements for a flawless construction in terms of building physics are very high. Hygrothermal risks, structural risks, robustness, and thermal bridges must be examined in detail.

Based on the solutions for the wall constructions, it is obvious that there are very high hygrothermal risks, especially with regard to internal insulation. Therefore, it is not surprising that 6 out of the 11 best practice examples which reach $U$-values below $0.15 \mathrm{~W} /\left(\mathrm{m}^{2} \cdot \mathrm{K}\right)$ have been applied external insulation. However, there are also individual solutions with internal insulation where, through simulation and on-site measurements, mould growth can be ruled out and high energy efficiency is achieved.

At the Osramhuset, in addition to the solution of an external insulation, an internal insulation that achieves a $U$ value of $0.13 \mathrm{~W} / \mathrm{m}^{2} \cdot \mathrm{K}$ was implemented by means of a consistent vapour barrier. Excellent values were also achieved with Perlite insulation and aerogel in the Villa Castelli. In the case of the Villa Castelli, the application of this solution should not be generalized. The solution works perfectly for the location in Italy, but would probably lead to damage in high alpine regions. At Ansitz Kofler, a rather critical internal insulation of mineral wool on a block wall was installed. This insulation works there due to sufficient driving rain protection and the geographical location. The choice of a capillary-active insulation material would be advantageous in this case.

For the remaining solutions, lower insulation thicknesses were mostly used to avoid the mould problem without detailed analysis.

The problem of thermal bridges was also considered in detail for some buildings. In case of refurbishments with external insulation, remaining thermal bridges mostly only caused higher heat losses. In case of internal insulation, they can quickly lead to moisture damage. Wherever possible, at the "Ansitz Kofler" the internal wall was cut and insulation placed vertically against the wall. Where new partition walls were built up, interspaces to the external wall were left in order to place insulation, OSB-boarding and vapor retarder behind. Thus, the energy losses as well as possible moisture damage, caused by the thermal bridge of inner walls, were avoided. 


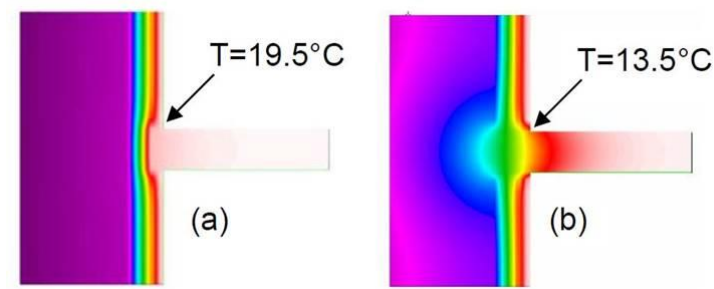

Figure 2:2-dimensional simulation of partition wall abutting outside wall with THERM a) thermal division b) no thermal division

Due to the renovation, new floor constructions and thus higher payloads are usually applied to the intermediate ceilings. These additional loads must be absorbed by the existing walls. Especially in the case of wooden block buildings, additional subsidence is to be expected and must be considered.

\subsubsection{Heritage compatibility}

As already mentioned, in most cases an internal insulation was installed. This fulfilled the requirements for maintaining the original appearance and did not change the proportions of the building. Sometimes, the plastering on the façade was renewed. Here, great importance was attached to the use of suitable plasters such as lime plasters. Particularly at the "Ruckzaunerhof", the restoration of the exterior plaster was carried out in close cooperation with the Department of Monuments in order to preserve the old plaster.

External insulation was mostly used for unlisted buildings and for building components that were not subject to any requirements by the heritage agency. But even there, the preservation of proportions was considered. At the Mariahilferstraße 182, the outer walls were moved inwards to make space for a $20 \mathrm{~cm}$ thick hemp insulation.

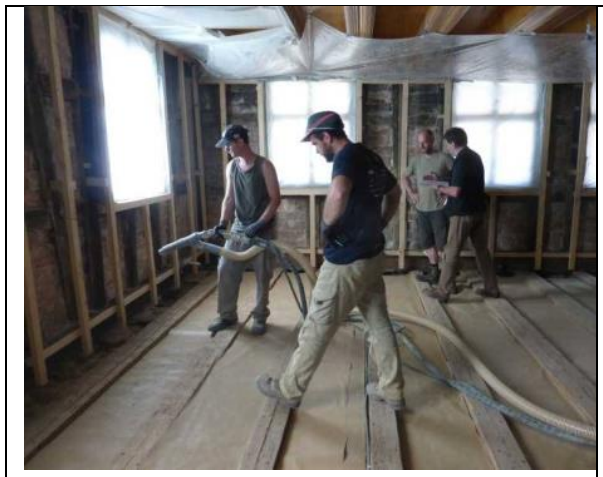

Figure 3: timber framed house in Alsace, sprayed hemp concrete

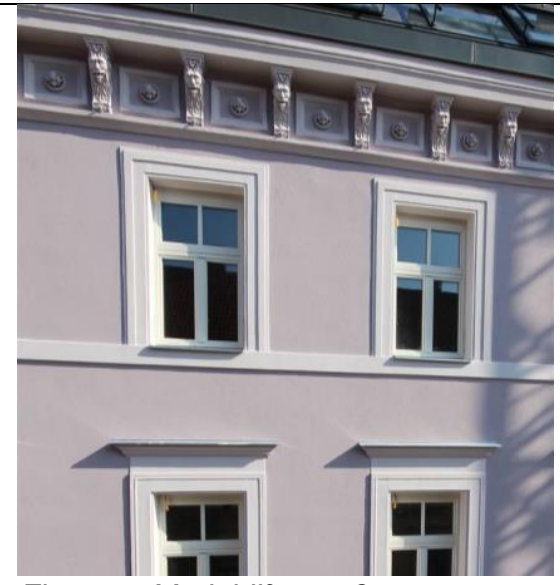

Figure 6: Mariahilferstraße 182, aerogel facade

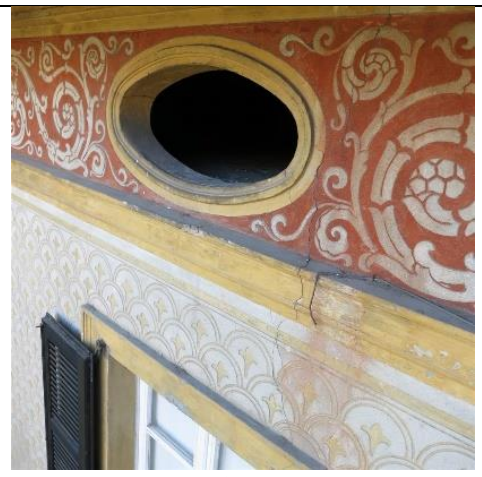

Figure 4: Villa Castelli, historical plaster and painting

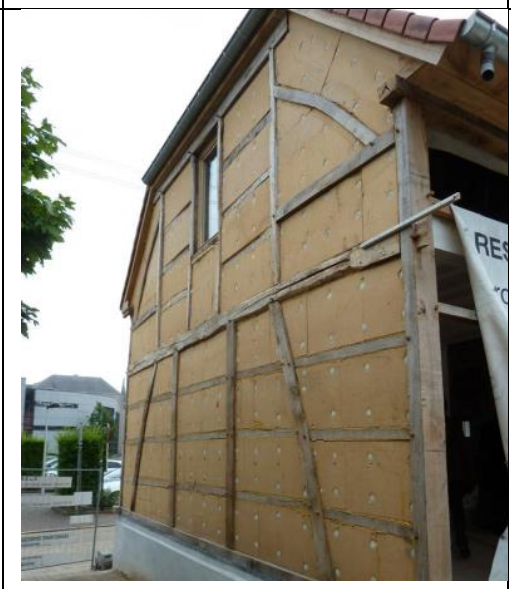

Figure 7: half-timbered house in Alkjen, wood fibre board with clay plaster

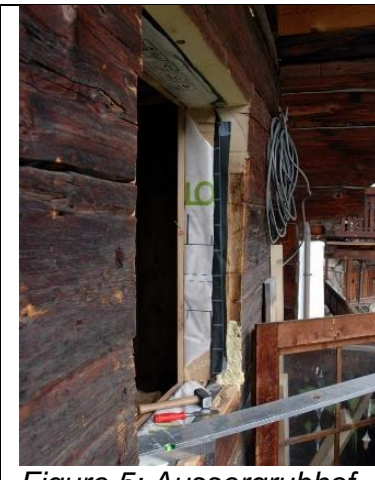

Figure 5: Aussergrubhof, internal hemp fibre insulation

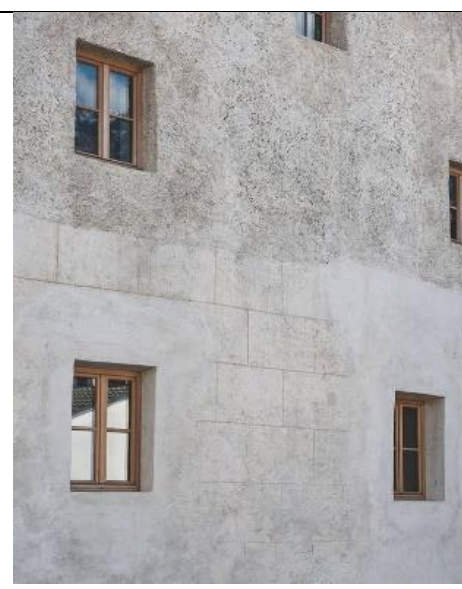

Figure 8: Ruckzaunerhof, historic plaster from the $16^{\text {th }}$ century 


\subsubsection{Roof}

\subsubsection{Overview of roof-solutions}

Out of 69 best practice examples, 45 roofs were refurbished and documented (see Table 10). The roof shapes were retained in all cases and were evident in 61 examples.

Table 10: Overview of roof-solutions

\begin{tabular}{|c|c|c|c|c|c|}
\hline \multirow[t]{2}{*}{$\stackrel{0}{\mathbf{z}}$} & \multirow[t]{2}{*}{ 点 출 } & \multirow[t]{2}{*}{ 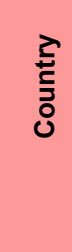 } & \multirow[t]{2}{*}{ 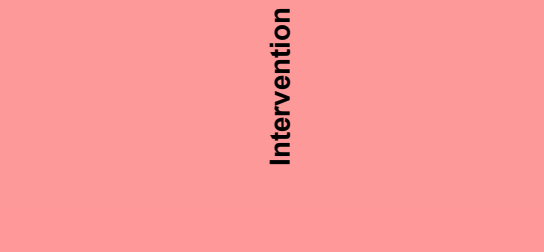 } & \multicolumn{2}{|c|}{ 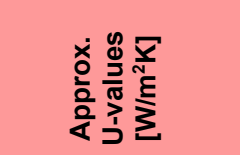 } \\
\hline & & & & Before & After \\
\hline 1 & Klostergebäude Kaiserstrasse & AT & Hipped roof - insulation between rafters & 0.90 & 0.16 \\
\hline 2 & Farm house Trins & AT & $\begin{array}{l}\text { New pitched roof - insulation between } \\
\text { rafters }\end{array}$ & & 0.12 \\
\hline 3 & Hof 6, Schwarzenberg, Voralberg & AT & $\begin{array}{l}\text { New pitched roof - insulation between } \\
\text { rafters }\end{array}$ & 4.00 & 0.28 \\
\hline 4 & Kelchalm - Bochumer alpine hut & AT & Hipped roof - insulation between rafters & 1.65 & 0.15 \\
\hline 5 & Mariahilferstrasse 182 & AT & New partly pent roof & - & 0.30 \\
\hline 6 & House Maurer, Wolfurt & AT & $\begin{array}{l}\text { New pitched roof - insulation between } \\
\text { rafters }\end{array}$ & 0.89 & 0.15 \\
\hline 7 & House Breuer, Tschagguns & AT & $\begin{array}{l}\text { New pitched roof - insulation between } \\
\text { rafters }\end{array}$ & 2.50 & 0.13 \\
\hline 8 & Music school in Velden & AT & Pitched roof - insulation between rafters & 2.10 & 0.09 \\
\hline 9 & Hof Neuhäusl & AT & Pitched roof - insulation above rafters & - & 0.16 \\
\hline 10 & Community Hall Zwischenwasser & AT & Pitched roof - insulation between rafters & 3.00 & 0.15 \\
\hline 11 & Freihof Sulz & AT & Hipped roof - insulation on the attic floor & & 0.14 \\
\hline 12 & Oeconomy building Josef Weiss & AT & Pitched roof - insulation between rafters & 2.00 & 0.19 \\
\hline 13 & Giatla Haus & AT & $\begin{array}{l}\text { New pitched roof - insulation between } \\
\text { rafters }\end{array}$ & 2.80 & 0.11 \\
\hline 14 & Rhine Valley House Irgang & AT & Pitched roof - insulation between rafters & 3.00 & 0.18 \\
\hline 15 & Baur Residence, Lustenau & AT & $\begin{array}{l}\text { New pitched roof - insulation between } \\
\text { rafters }\end{array}$ & 1.42 & 0.18 \\
\hline 16 & Kasperhof & AT & Pitched roof - insulation between rafters & 2.97 & 0.18 \\
\hline 17 & Maison Rubens & $\mathrm{BE}$ & Pitched roof - insulation between rafters & - & 0.23 \\
\hline 18 & $\begin{array}{l}\text { Half-timberframed house in Alken, } \\
\text { Belgium }\end{array}$ & $\mathrm{BE}$ & $\begin{array}{l}\text { Hipped roof - insulation above old roof } \\
\text { truss and } \\
\text { between the new rafters }\end{array}$ & 5.07 & 0.26 \\
\hline 19 & Doragno Castle, Rovio & $\mathrm{CH}$ & New pent roof - inslation abover rafters & 1.00 & 0.20 \\
\hline 20 & Solar silo & $\mathrm{CH}$ & New pent roof - inslation abover rafters & 2.00 & 0.19 \\
\hline 21 & $\begin{array}{l}\text { Wohn- und Geschäftshaus } \\
\text { Feldbergstrasse }\end{array}$ & $\mathrm{CH}$ & $\begin{array}{l}\text { New pitched roof - insulation between } \\
\text { rafters }\end{array}$ & 2.00 & 0.10 \\
\hline 22 & Mehrfamilienhaus Magnusstrasse & $\mathrm{CH}$ & $\begin{array}{l}\text { New ptiched / flat roof - insulation between } \\
\text { rafters }\end{array}$ & 2.00 & 0.13 \\
\hline 23 & St. Franziskus Church Ebmatingen & $\mathrm{CH}$ & Pitched roof - insulation between rafters & 0.26 & 0.12 \\
\hline 24 & $\begin{array}{l}\text { Kindergarten and apartments (PEB) } \\
\text { Chur }\end{array}$ & $\mathrm{CH}$ & $\begin{array}{l}\text { New pitched roof - insulation between } \\
\text { rafters }\end{array}$ & 3.00 & 0.12 \\
\hline 25 & $\begin{array}{l}\text { Single family home Luisenstrasse - } \\
\text { Bern }\end{array}$ & $\mathrm{CH}$ & Hipped roof - insulation between rafters & 1.36 & 0.19 \\
\hline 26 & Single Family House - Gstaad & $\mathrm{CH}$ & Hipped roof - insulation between rafters & - & 0.17 \\
\hline 27 & Glaserhaus in Affoltern & $\mathrm{CH}$ & Hipped roof - insulation above rafters & 3.00 & 0.16 \\
\hline 28 & PalaCinema Locarno & $\mathrm{CH}$ & Addition of a storey & - & 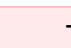 \\
\hline 29 & Casa Rossa Chemnitz & $\mathrm{DE}$ & $\begin{array}{l}\text { New pitched roof - insulation between } \\
\text { rafters }\end{array}$ & 5.00 & 0.12 \\
\hline 30 & Rathaus Bergrheinfeld & $\mathrm{DE}$ & Hipped roof - insulation on the attic floor & - & 0.23 \\
\hline
\end{tabular}




\begin{tabular}{|c|c|c|c|c|c|}
\hline 31 & Farmhouse Straub & DE & $\begin{array}{l}\text { Partly new pitched roof - insulation above } \\
\text { the rafters }\end{array}$ & 3.50 & 0.14 \\
\hline 32 & Early work Sep Ruf & $\mathrm{DE}$ & $\begin{array}{l}\text { Pitched roof - insulation between and on } \\
\text { rafters }\end{array}$ & 1.16 & 0.23 \\
\hline 33 & Ackerbürgerhäuschen & DE & New pitched roof above old roof truss & 3.70 & 0.22 \\
\hline 34 & Ritterhof & DE & $\begin{array}{l}\text { New pitched roof (1989) - insulation } \\
\text { between rafters }\end{array}$ & - & 0.20 \\
\hline 35 & Rathaus Burgkunstadt & $\mathrm{DE}$ & $\begin{array}{l}\text { Insulation of the attic floor with mineral } \\
\text { wool }\end{array}$ & - & 0.22 \\
\hline 36 & Osramhuset & DK & - & - & - \\
\hline 37 & Klitgaarden & DK & Pitched roof - insulation between rafters & - & - \\
\hline 38 & RYESGADE 30 & DK & Addition of a storey & - & - \\
\hline 39 & Timber-framed house in Alsace & FR & Hipped roof - insulation on the attic floor & 0.95 & 0.16 \\
\hline 40 & Elementary School in Mulhouse & FR & $\begin{array}{l}\text { Insulation of the attic floor with mineral } \\
\text { wool }\end{array}$ & 0.95 & 0.12 \\
\hline 41 & $\begin{array}{l}\text { Timber-framed barn in the north of } \\
\text { France }\end{array}$ & FR & Insulation of the attic floor with hemp wool & - & 0.14 \\
\hline 42 & Rainhof & IT & Pitched roof - insulation between rafters & 2.7 & 0.2 \\
\hline 43 & Villa Castelli & IT & $\begin{array}{l}\text { New hipped roof - insulation between and } \\
\text { above the rafters }\end{array}$ & 2.23 & 0.13 \\
\hline 44 & Ansitz Kofler & IT & Flat roof - insulation between rafters & 1.27 & 0.17 \\
\hline 45 & $\begin{array}{l}\text { Basilica di Santa Maria di } \\
\text { Collemaggio }\end{array}$ & IT & - & - & - \\
\hline 46 & House Pernter & IT & Insulation on the attic floor with XPS & - & - \\
\hline 47 & Kohlerhaus & IT & Hipped roof - insulation between rafters & 2.30 & 0.19 \\
\hline 48 & Ruckenzaunerhof & IT & Pitched roof - insulation between rafters & 3.09 & 0.43 \\
\hline 49 & Aussergrubhof & IT & Pitched roof - insulation above rafters & - & 0.22 \\
\hline 50 & Oberbergerhof & IT & Insulation on the attic floor & 1.00 & 0.2 \\
\hline 51 & Platzbonhof & IT & Pitched roof - insulation above rafters & 1.22 & 0.17 \\
\hline 52 & Mairhof & IT & $\begin{array}{l}\text { New pitched roof - insulation between } \\
\text { rafters }\end{array}$ & 1.22 & 0.13 \\
\hline 53 & Obergasserhof & IT & New pitched roof - insulation above rafters & - & 0.16 \\
\hline 54 & Rebecco Farm & IT & New pitched roof - insulation above rafters & 0.52 & 0.17 \\
\hline 55 & Villa Capodivacca & IT & Tent roof - insulation above rafters & 2.38 & 0.22 \\
\hline 56 & House Moroder & IT & Insulation on the attic floor (20 cm EPS) & - & - \\
\hline 57 & Huberhof & IT & Pitched roof - insulation between rafters & 0.81 & 0.22 \\
\hline 58 & Notarjeva vila & SL & Tent roof - insulation between rafters & 1.50 & 0.15 \\
\hline 59 & Rožna ulica 15, Idrija & SL & - & 3.30 & 0.15 \\
\hline 60 & Hiša trentarskih vodnikov & SL & Hipped roof - insulation between rafters & 8.00 & 0.15 \\
\hline 61 & Idrija mercury smelting plant & SL & - & - & - \\
\hline 62 & Mercado del Val, Valladolid & SP & New pitched roof - insulation above rafters & 1.07 & 0.38 \\
\hline 63 & Correria 119 & SP & Pitched roof & - & - \\
\hline 64 & Ahmet Aga Mansion & TK & only repairing the pitched roof & & \\
\hline 65 & Nwcip Pasa Library & TK & - & - & - \\
\hline 66 & Downie's Cottage & UK & Insulation on the attic floor & - & - \\
\hline 67 & Hollyrood Park Lodge & UK & Pitched roof - insulation between rafters & 1.08 & 0.36 \\
\hline 68 & Annat Road & UK & Hipped roof - insulation between rafters & 1 & 0.13 \\
\hline 69 & Aspinall Courthouse & USA & Flat roof & - & - \\
\hline
\end{tabular}




\subsubsection{Roof shape and measures}

Many different roof shapes are included in the 45 examples. The most common roof form is the pitched roof with 38 out of 61 examples. Hipped roofs were renovated in 16 cases. Furthermore, there are three pent roofs, two tent roofs and also two flat roofs in the collection.

The thermal upgrading was achieved by various measures. In 23 cases, insulation between the rafters was carried out. In some cases, the rafters were doubled to achieve a higher insulation thickness. Different insulation materials such as mineral wool, glass wool, cellulose and wood fibre insulation were used. Especially wood fibre insulation was used in many cases. For the "Villa Castelli" and the "Mercado del Val" a mixture of between-rafter insulation and above-rafter insulation was installed. In eight other cases a simple above-rafter insulation was used. It is not always possible to insulate the outer roof level. Therefore, in 10 cases the ceiling of the attic was insulated. In the case of the elementary school "Cour de Larraine" $260 \mathrm{~mm}$ mineral wool was applied to the attic of the last floor.

Throughout, the thermal improvement was quite huge. For example, the U-values of $0.26-5.07 \mathrm{~W} /\left(\mathrm{m}^{2} \cdot \mathrm{K}\right) \mathrm{were}$ improved to a range of $0.09-0.43 \mathrm{~W} /\left(\mathrm{m}^{2} \cdot \mathrm{K}\right)$ by the additional insulation. In 14 of the 69 buildings $\mathrm{U}$-values of even $\leq 0.15 \mathrm{~W} / \mathrm{m}^{2} \mathrm{~K}$ were achieved.

\subsubsection{Technical compatibility}

The technical requirements for roofs are not as complex as for wall solutions with internal insulation. In most cases, conventional roof structures are used. In the case of insulation between rafters, moderate vapour barriers minimize the entry of moisture and diffusion-open underlay membranes and possibly rear ventilation allow excess moisture to be removed. In this context, the roof must be refurbished as a complete system. Partial refurbishment can lead to considerable damage if carried out incorrectly. For example, insulation between the rafters in combination with an old diffusion-tight roof sheeting would be catastrophic and could destroy a roof truss within a few years.

\subsubsection{Heritage compatibility}

In order to meet the visual requirements of the monument protection, insulation between rafters is a good solution. The proportions of the building remain unchanged and the roof covering can be retained. Also, with other roof insulation solutions the original roof covering can be reused or reproduced. For example, the "Rainhof" (figure 9) was covered with long shingles, the "Klostergebäude Kaiserstraße" (figure 14) and the "Hollyrood Park Lodge" (figure 12) were covered with the original slate tiles. In the case of the "Klitgaarden", the historic thatched roof was completely preserved and thermally improved with additional insulation between the rafters.

In some cases, parts of the roof truss are damaged so badly that renovation is almost impossible. In 22 cases the roof truss was therefore completely renewed. As some examples show, this is also possible for listed buildings while retaining the roof shape and the materials used.

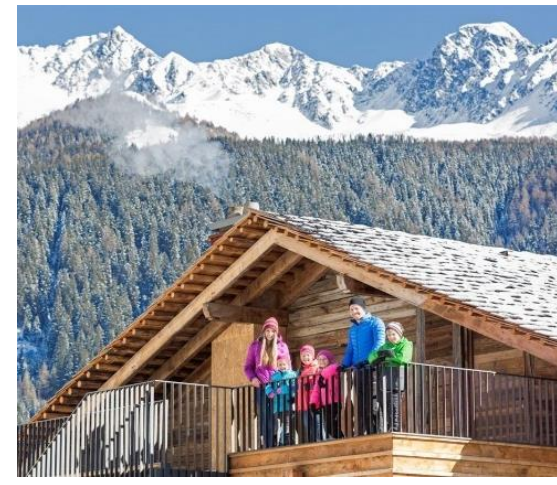

Figure 9: Rainhof, roof with long shingles and insulation between rafters

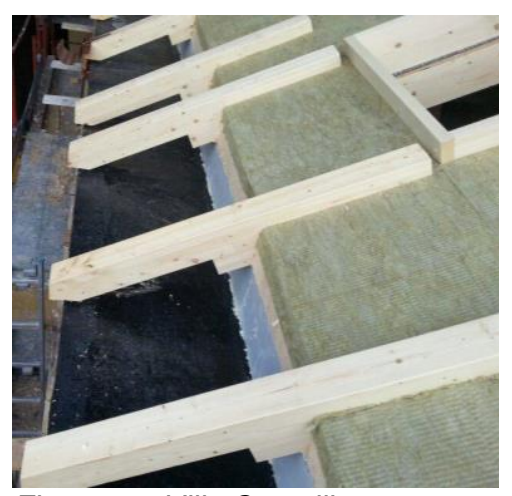

Figure 10: Villa Castelli, new pitched roof

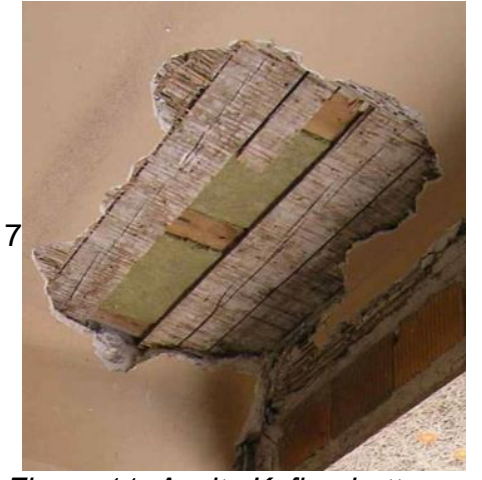

Figure 11: Ansitz Kofler, bottom view of the insulation between the rafters 


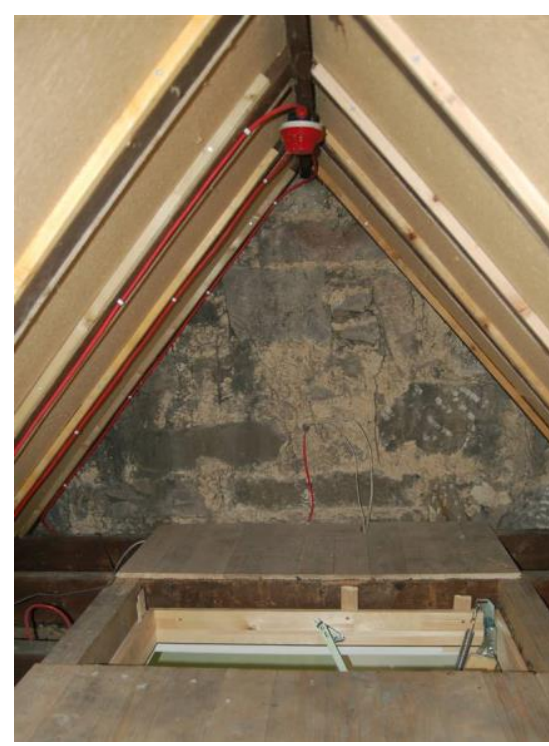

Figure 12: Hollyrood Park Lodge, pitched roof with wood fibre

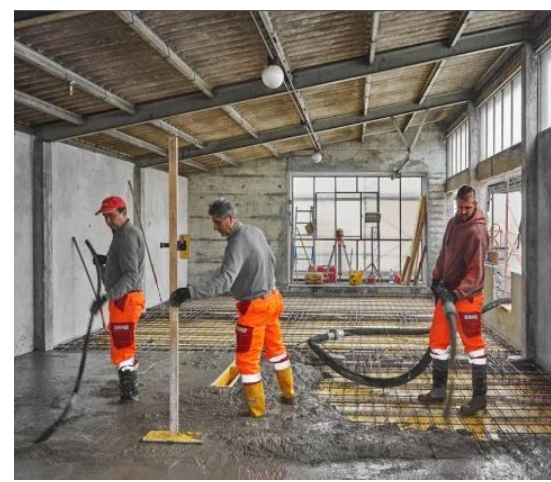

Figure 15: Solar Silo, old beam structure

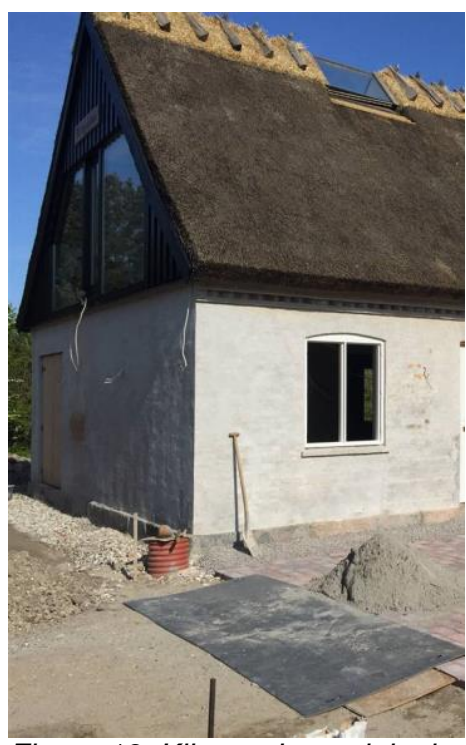

Figure 13: Klitgaarden, original thatched roof with internal insulation between the rafters

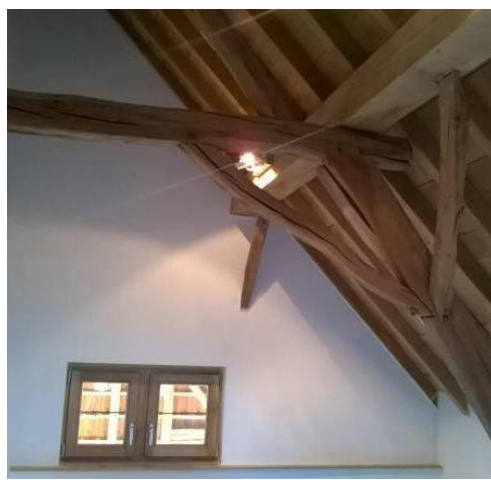

Figure 16: Half timberframed house in Alken, old roof truss with some new elements

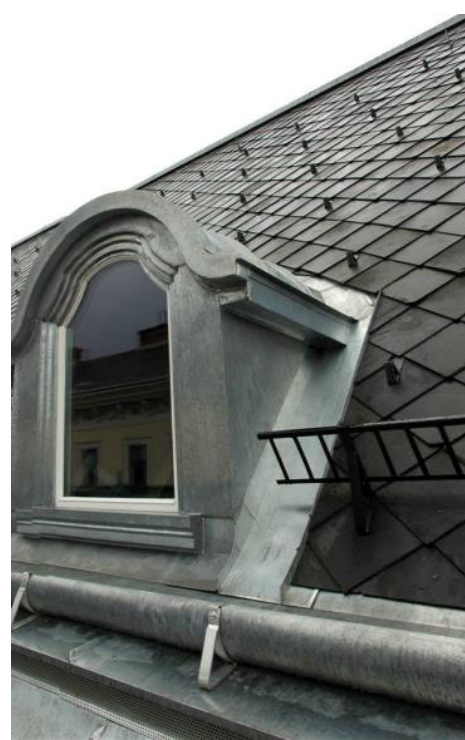

Figure 14: Klostergebäude

Kaiserstraße, roofing with original slate tiles

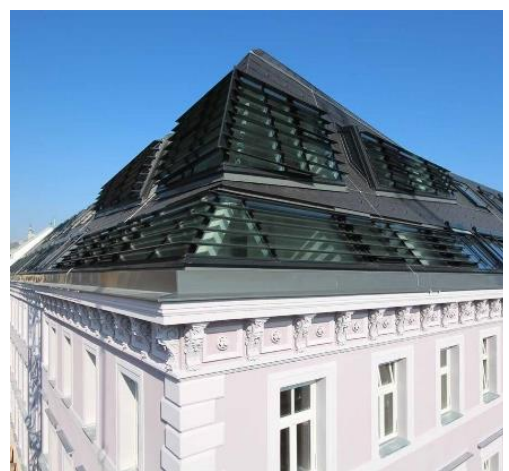

Figure 17: Mariahilferstraße 182, modern roof solution 


\subsubsection{Floor}

\subsubsection{Overview of floor-solutions}

As table 11 shows, in 45 of the 69 best practice examples, the refurbishment of the ground floor was documented.

Table 11: Overview of floor-solutions

\begin{tabular}{|c|c|c|c|c|c|}
\hline \multirow[t]{2}{*}{ i } & \multirow[t]{2}{*}{ 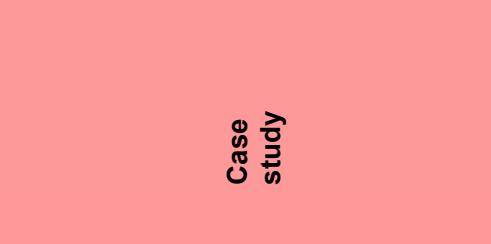 } & \multirow[t]{2}{*}{ 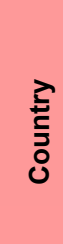 } & \multirow[t]{2}{*}{ 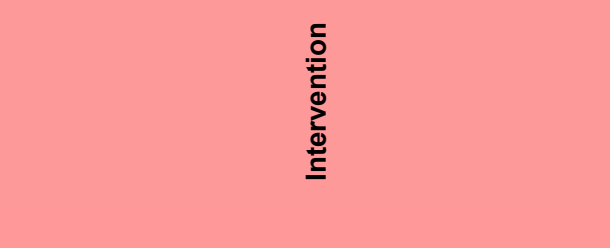 } & \multicolumn{2}{|c|}{ 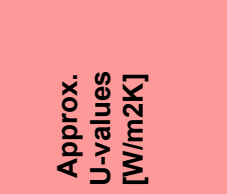 } \\
\hline & & & & Before & After \\
\hline 1 & Klostergebäude Kaiserstrasse & AT & - & - & - \\
\hline 2 & Farm house Trins & AT & - & - & - \\
\hline 3 & $\begin{array}{l}\text { Hof 6, Schwarzenberg, Voralberg, } \\
\text { Austria }\end{array}$ & AT & $\begin{array}{l}\text { Adding insulation on the wood beam ceiling of } \\
\text { the cellar }\end{array}$ & 2.50 & 0.17 \\
\hline 4 & Kelchalm - Bochumer alpine hut & AT & - & - & - \\
\hline 5 & Mariahilferstrasse 182 & AT & - & - & - \\
\hline 6 & House Maurer, Wolfurt & AT & $\begin{array}{l}\text { Adding EPS insulation above the new concrete } \\
\text { slab }\end{array}$ & 0.68 & 0.20 \\
\hline 7 & House Breuer, Tschagguns & AT & $\begin{array}{l}\text { Adding } 20 \mathrm{~cm} \text { of EPS insulation above the new } \\
\text { concrete slab }\end{array}$ & 1.70 & 0.17 \\
\hline 8 & Music school in Velden & AT & $\begin{array}{l}\text { Adding } 4 \mathrm{~cm} \text { of XPS above existing concrete } \\
\text { slab }\end{array}$ & 4.00 & 0.59 \\
\hline 9 & Hof Neuhäusl & AT & $\begin{array}{l}\text { Adding } 22 \mathrm{~cm} \text { of PU - insulation above new } \\
\text { concrete slab }\end{array}$ & 2.00 & 0.10 \\
\hline 10 & Community Hall Zwischenwasser & AT & $\begin{array}{l}\text { Adding } 16 \mathrm{~cm} \text { of mineral wool insulation } \\
\text { underneath existing concrete ceiling }\end{array}$ & 1.80 & 0.14 \\
\hline 11 & Freihof Sulz & AT & - & - & - \\
\hline 12 & Oeconomy building Josef Weiss & AT & $\begin{array}{l}\text { Adding } 20 \mathrm{~cm} \text { of XPS underneath the new } \\
\text { concrete slab }\end{array}$ & 2.30 & 0.18 \\
\hline 13 & Giatla Haus & AT & Adding $20 \mathrm{~cm}$ of EPS above new concrete slab & 2.80 & 0.17 \\
\hline 14 & Rhine Valley House Irgang & AT & $\begin{array}{l}\text { Adding } 15 \mathrm{~cm} \text { of glass wool underneath the } \\
\text { floorboards }\end{array}$ & 3.00 & 0.17 \\
\hline 15 & Baur Residence, Lustenau & AT & - & - & - \\
\hline 16 & Kasperhof & AT & $\begin{array}{l}15 \mathrm{~cm} \text { of foam glass gravel underneath a } 5 \mathrm{~cm} \\
\text { granular subbase }\end{array}$ & 2.50 & 0.24 \\
\hline 17 & Maison Rubens & $\mathrm{BE}$ & $\begin{array}{l}\text { Adding blow in cellulose underneath the brick } \\
\text { arches }\end{array}$ & - & 0.19 \\
\hline 18 & $\begin{array}{l}\text { Half-timberframed house in Alken, } \\
\text { Belgium }\end{array}$ & $\mathrm{BE}$ & $\begin{array}{l}\text { Adding } 6 \mathrm{~cm} \text { of PUR - insulation above new } \\
\text { concrete slab }\end{array}$ & 0.76 & 0.30 \\
\hline 19 & Doragno Castle, Rovio (CH) & $\mathrm{CH}$ & $\begin{array}{l}\text { Adding } 12 \mathrm{~cm} \text { of insulation underneath new } \\
\text { concrete slab }\end{array}$ & 0.90 & 0.25 \\
\hline 20 & Solar silo & $\mathrm{CH}$ & - & - & - \\
\hline 21 & $\begin{array}{l}\text { Wohn- und Geschäftshaus } \\
\text { Feldbergstrasse }\end{array}$ & $\mathrm{CH}$ & $\begin{array}{l}\text { Adding insulation on the wood beam ceiling of } \\
\text { the cellar }\end{array}$ & 2.40 & 0.15 \\
\hline 22 & Mehrfamilienhaus Magnusstrasse & $\mathrm{CH}$ & $\begin{array}{l}\text { Adding insulation on the wood beam ceiling of } \\
\text { the cellar }\end{array}$ & 2.00 & 0.18 \\
\hline 23 & St. Franziskus Church Ebmatingen & $\mathrm{CH}$ & - & - & - \\
\hline 24 & $\begin{array}{l}\text { Kindergarten and apartments (PEB) } \\
\text { Chur }\end{array}$ & $\mathrm{CH}$ & $\begin{array}{l}\text { Adding } 50 \mathrm{~cm} \text { of foam glass gravel underneath } \\
\text { new concrete slab }\end{array}$ & 3.50 & 0.16 \\
\hline 25 & $\begin{array}{l}\text { Single family home Luisenstrasse - } \\
\text { Bern }\end{array}$ & $\mathrm{CH}$ & $\begin{array}{l}\text { Adding } 14 \mathrm{~cm} \text { of mineral wool underneath the } \\
\text { concrete ceiling }\end{array}$ & 1.00 & 0.19 \\
\hline 26 & $\begin{array}{l}\text { Single Family House - Gstaad, } \\
\text { Switzerland }\end{array}$ & $\mathrm{CH}$ & $\begin{array}{l}\text { Adding } 18 \mathrm{~cm} \text { of XPS underneath new concrete } \\
\text { slab }\end{array}$ & - & 0.17 \\
\hline 27 & Glaserhaus in Affoltern & $\mathrm{CH}$ & $\begin{array}{l}\text { Adding } 15 \mathrm{~cm} \text { of XPS underneath new concrete } \\
\text { slab }\end{array}$ & 1.00 & 0.16 \\
\hline 28 & PalaCinema Locarno & $\mathrm{CH}$ & $\begin{array}{l}\text { Adding } 20 \mathrm{~cm} \text { of insulation above new concrete } \\
\text { slab }\end{array}$ & - & - \\
\hline 29 & Casa Rossa Chemnitz & $\mathrm{DE}$ & $\begin{array}{l}\text { Adding } 8 \mathrm{~cm} \text { of perlite gravel above existing } \\
\text { ceiling of the cellar }\end{array}$ & - & - \\
\hline
\end{tabular}




\begin{tabular}{|c|c|c|c|c|c|}
\hline 30 & Rathaus Bergrheinfeld & $\mathrm{DE}$ & $\begin{array}{l}\text { Adding } 6 \mathrm{~cm} \text { of perlite gravel above existing } \\
\text { ceiling of the cellar }\end{array}$ & - & 0.35 \\
\hline 31 & Farmhouse Straub & $\mathrm{DE}$ & $\begin{array}{l}\text { Adding } 20 \mathrm{~cm} \text { of blow in cellulose between wood } \\
\text { beam ceiling of the cellar }\end{array}$ & 1.20 & 0.18 \\
\hline 32 & Early work Sep Ruf & $\mathrm{DE}$ & $\begin{array}{l}\text { Adding } 8 \mathrm{~cm} \text { of EPS underneath the concrete } \\
\text { ceiling of the cellar }\end{array}$ & 1.20 & 0.32 \\
\hline 33 & Ackerbürgerhäuschen & $\mathrm{DE}$ & $\begin{array}{l}\text { Adding } 6 \mathrm{~cm} \text { of insulation above new concrete } \\
\text { slab and } 25 \mathrm{~cm} \text { of foam glass gravel underneath. }\end{array}$ & - & 0.21 \\
\hline 34 & Ritterhof & $\mathrm{DE}$ & Adding insulation above new concrete slab & - & 0.20 \\
\hline 35 & Rathaus Burgkunstadt & $\mathrm{DE}$ & - & - & - \\
\hline 36 & Osramhuset (The Osram Building) & DK & - & - & - \\
\hline 37 & Klitgaarden & DK & $\begin{array}{l}\text { Adding } 10 \mathrm{~cm} \text { of insulation on existing concrete } \\
\text { slab }\end{array}$ & - & - \\
\hline 38 & RYESGADE 30 & DK & - & - & - \\
\hline 39 & $\begin{array}{l}\text { Timber-framed house in Alsace, } \\
\text { France }\end{array}$ & $\mathrm{FR}$ & $\begin{array}{l}\text { Adding } 13 \mathrm{~cm} \text { of lime-hemp concrete between } \\
\text { new joists }\end{array}$ & 0.95 & 0.4 \\
\hline 40 & $\begin{array}{l}\text { Elementary School in Mulhouse, } \\
\text { France }\end{array}$ & FR & Adding $8 \mathrm{~cm}$ of PUR - insulation & 1.7 & 0.35 \\
\hline 41 & $\begin{array}{l}\text { Timber-framed barn in the north of } \\
\text { France }\end{array}$ & $\mathrm{FR}$ & Adding $20 \mathrm{~cm}$ of polysterol & - & - \\
\hline 42 & Rainhof & IT & Adding $7.5 \mathrm{~cm}$ of insulation & 3.21 & 0.28 \\
\hline 43 & Villa Castelli & IT & Adding $20 \mathrm{~cm}$ of XPS above new concrete slab & 1.49 & 0.15 \\
\hline 44 & Ansitz Kofler & IT & $\begin{array}{l}\text { Adding } 20 \mathrm{~cm} \text { of XPS above existing concrete } \\
\text { slab }\end{array}$ & 2.42 & 0.17 \\
\hline 45 & $\begin{array}{l}\text { Basilica di Santa Maria di } \\
\text { Collemaggio }\end{array}$ & IT & - & - & - \\
\hline 46 & House Pernter & IT & $\begin{array}{l}\text { Adding } 20 \mathrm{~cm} \text { of XPS underneath the new } \\
\text { concrete slab }\end{array}$ & - & - \\
\hline 47 & Kohlerhaus & IT & $\begin{array}{l}\text { Adding } 5 \mathrm{~cm} \text { of foam glass on the existing } \\
\text { concrete slab }\end{array}$ & 3 & 1.1 \\
\hline 48 & Ruckenzaunerhof & IT & $\begin{array}{l}\text { Adding } 10 \mathrm{~cm} \text { of insulation above new concrete } \\
\text { slab }\end{array}$ & - & 0.28 \\
\hline 49 & Aussergrubhof & IT & $\begin{array}{l}\text { Adding } 4 \mathrm{~cm} \text { of Polyfoam insulation and } 5 \mathrm{~cm} \text { of } \\
\text { Liapor gravel on the wooden beam ceiling }\end{array}$ & - & 0.38 \\
\hline 50 & Oberbergerhof & IT & - & - & - \\
\hline 51 & Platzbonhof & IT & $\begin{array}{l}\text { Adding insulation on the wood beam ceiling of } \\
\text { the cellar }\end{array}$ & 6.55 & 0.28 \\
\hline 52 & Mairhof & IT & $\begin{array}{l}\text { Adding insulation above the existing concrete } \\
\text { slab }\end{array}$ & 3.23 & 0.26 \\
\hline 53 & Obergasserhof & IT & Adding insulation above new concrete slab & - & 0.37 \\
\hline 54 & Rebecco Farm & IT & - & - & - \\
\hline 55 & Villa Capodivacca & IT & - & - & - \\
\hline 56 & House Moroder & IT & - & - & - \\
\hline 57 & Huberhof & IT & Adding $12 \mathrm{~cm}$ XPS above new concrete slab & - & - \\
\hline 58 & Notarjeva vila & $\mathrm{SL}$ & - & - & - \\
\hline 59 & Rožna ulica 15, Idrija & SL & - & 2.8 & 0.27 \\
\hline 60 & Hiša trentarskih vodnikov & SL & - & - & - \\
\hline 61 & Idrija mercury smelting plant & $\mathrm{SL}$ & - & - & - \\
\hline 62 & Mercado del Val, Valladolid (Spain) & SP & New construction & 0.507 & 0.36 \\
\hline 63 & Correria 119 & SP & - & - & - \\
\hline 64 & Ahmet Aga Mansion & TK & only repairing the floor & - & - \\
\hline 65 & Nwcip Pasa Library & TK & only repairing the floor with new terracotta tiles & - & - \\
\hline 66 & Downie's Cottage & UK & Insulated lime concrete floor & 4.5 & 0.28 \\
\hline 67 & Hollyrood Park Lodge & UK & $\begin{array}{l}\text { Adding wood fiber insulation between existing } \\
\text { joists }\end{array}$ & 1.04 & 0.22 \\
\hline 68 & Annat Road & UK & $\begin{array}{l}\text { Adding wood fiber insulation between existing } \\
\text { joists }\end{array}$ & - & - \\
\hline 69 & $\begin{array}{l}\text { Aspinall Courthouse - Net Zero } \\
\text { renovation }\end{array}$ & USA & - & - & - \\
\hline
\end{tabular}




\subsubsection{Measures}

The renovation of the floor structure was carried out in different ways. Basically, it is necessary to differentiate between cases where a concrete slab already exists and cases where a new concrete slab was made. In 10 out of 45 cases the insulation was applied to an existing floor structure. In 20 cases a new lean concrete slab was installed. In 6 of these 20 solutions the insulation was installed under the new reinforced concrete slab, in 14 solutions above it. A specially mentioned bitumen sealing was only found in 5 solutions. At this point, it is important to note that the information content of the documentation varies greatly.

At "Downie's Cottage" and "Hollyrood Park Lodge" the existing floor construction consisted of wooden beams. In order to increase the thermal resistance, insulation was placed between the beams. There are also 12 examples in the collection where the ceiling between the basement and ground floor was thermally upgraded.

Especially in connection with a reinforced concrete slab, XPS and PUR were mostly used as insulation material. For the wooden constructions materials as hemp concrete, mineral wool and wood fiber were chosen. The insulation measures result in a significant improvement of the thermal resistance, values between $0.10-1.10$ $\mathrm{W} /\left(\mathrm{m}^{2} \cdot \mathrm{K}\right)$ were achieved.

\subsubsection{Technical Compatibility}

As for the walls, the position of the insulation is also decisive for the floor construction. If the insulation is installed as internal insulation, the same rules as for the walls apply. However, it is necessary to differentiate the scope of the insulation, as the outside temperatures to the ground are not as low as for an external wall. For the "half timberframed house in Alken" a vapour retarder was installed above the insulation to minimise the entry of moisture onto the cold concrete slab.

A completely different solution was used for Downie's Cottage. There insulated lime conrete was applied. This allows a continuous flow of water vapour.

\subsubsection{Heritage Compatibility}

Especially with floor constructions many possibilities exist to reconcile energetic necessity and monument protection. In many cases, the existing floor coverings can be removed and later re-integrated into the new structure.

For example, at "Downie's Cottage" the original flagstone floor was reinstalled (figure 19). In the case of the "timber framed house in Alsace" the existing terracotta tiles were used again. Wooden floors can also be reused, as the example of "Hollyrood Park Lodge" shows. In figure 23 you can see the reinstallation of the quarry stones at the "Ansitz Mairhof".

Another possibility is the imitation of existing floors. In the case of the "Oberbergerhof" the existing terrazzo floor was reproduced (figure 22).

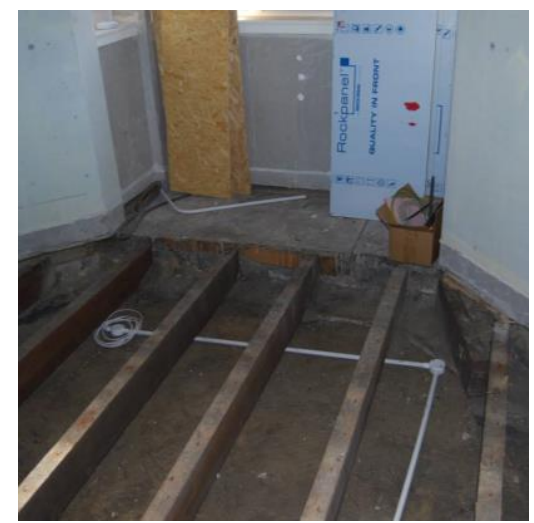

Figure 18: Hollyrood Park Lodge, suspended timber floors

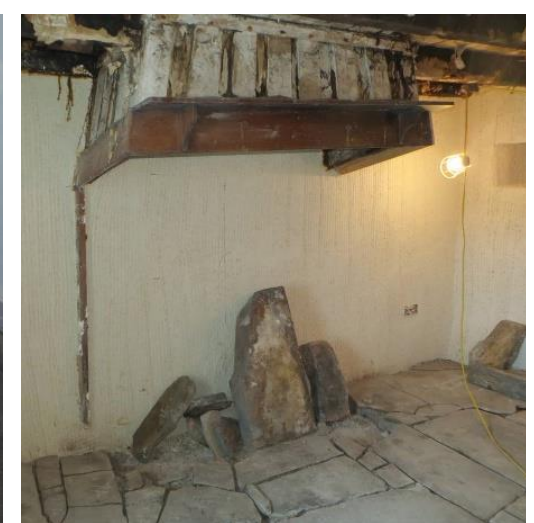

Figure 19: Downie's Cottage, original flagstone floor

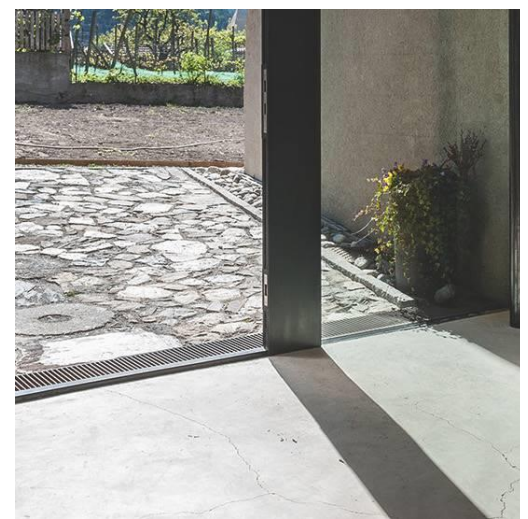

Figure 20: Ruckzaunerhof, new floor construction 


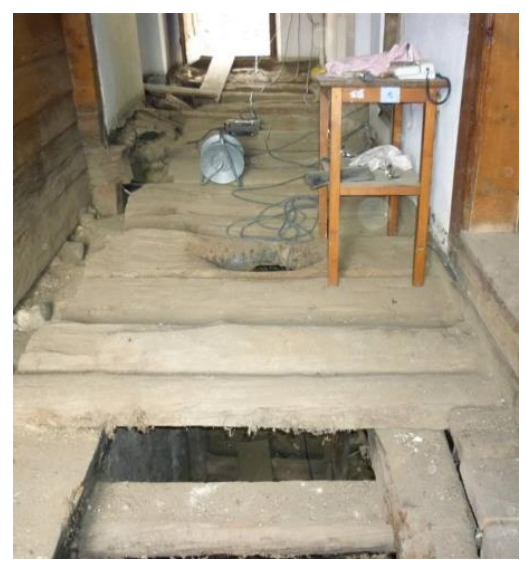

Figure 21: Aussergrubhof, old wooden floor joists

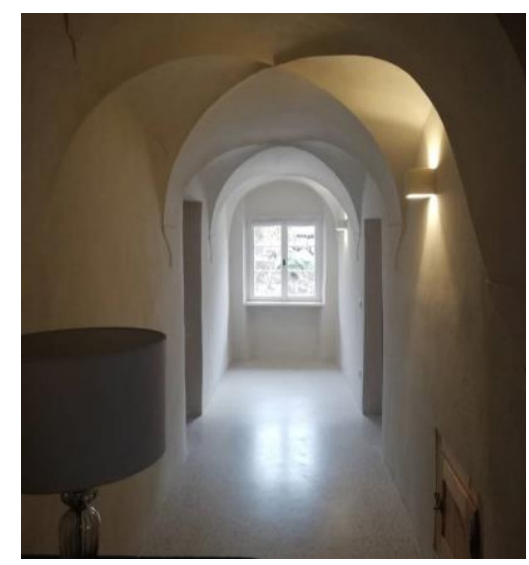

Figure 22: Oberbergerhof, imitated terrazzo floor

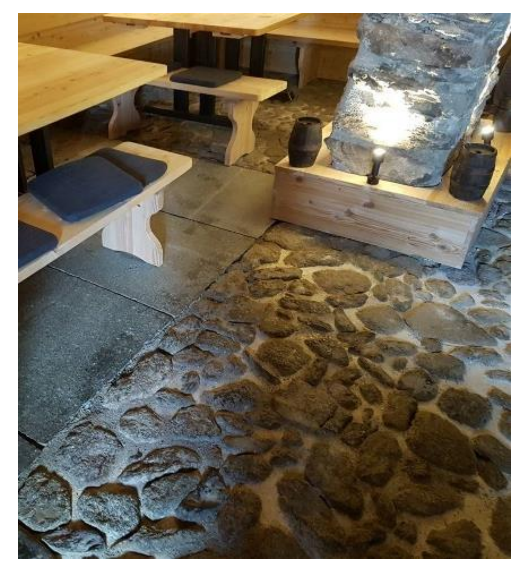

Figure 23: Ansitz Mairhof, historical floor made of quarry stones

\subsubsection{Windows}

\subsubsection{Overview of window solutions}

In general, all photos in this chapter are taken from the individual cases and they show solutions after renovation. Only few cases have registered the solar transmittance of the new/restored windows, however typically they are in the range of $g=0.45-0.50$ for triple-layer and $g=0.55-0.65$ for double-layer. Similar for light transmittance, no values have been registered.

In the 69 cases analysed, there are 67 different documented window solutions, i.e. not counting solutions where the only difference is e.g. layers of glazing. For instance, in Doragno Castle, most of the new windows have three layers of glass, but some have two layers - this is counted as one solution.

The solutions range from small repairs and careful restoration where maintaining the overall appearance of the building is vital, to replacing with or even adding new low energy windows bringing significant changes to the original appearance of the building. The following Table 12 gives an overview of the solutions.

Table 12: Types of window solutions in case studies.

\begin{tabular}{|c|c|c|c|c|c|c|}
\hline \multirow[t]{2}{*}{ No } & \multirow[t]{2}{*}{ Case study } & \multirow[t]{2}{*}{ 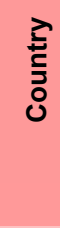 } & \multirow[t]{2}{*}{ Building type } & \multirow[t]{2}{*}{ Intervention } & \multicolumn{2}{|c|}{$\begin{array}{c}\text { Approx. } \\
\text { U-values }\left[\mathrm{W} / \mathrm{m}^{2} \mathrm{~K}\right]\end{array}$} \\
\hline & & & & & Before & After \\
\hline 1 & Klostergebäude Kaiserstrasse & AT & Residential & Renovate & 4.10 & 1.06 \\
\hline 2 & Farm house Trins & AT & Residential & Replace & 4.40 & 0.79 \\
\hline 3 & $\begin{array}{l}\text { Hof } 6 \text {, Schwarzenberg, Voralberg, } \\
\text { Austria }\end{array}$ & AT & Residential & Refurbish & 1.65 & 1.65 \\
\hline 4 & Kelchalm - Bochumer alpine hut & AT & Restaurant & Replace & 2.20 & 0.73 \\
\hline 5 & Mariahilferstrasse 182 & AT & Residential & & & \\
\hline 6 & House Maurer, Wolfurt & AT & Residential & Replace & 2.42 & 0.75 \\
\hline 7 & House Breuer, Tschagguns & AT & Residential & New & & 0.75 \\
\hline 8 & Music school in Velden & AT & Educational & Renovate & 2.50 & 0.82 \\
\hline 9 & Hof NeuhäusI & AT & Residential & New & 5.50 & 0.70 \\
\hline 10 & Community Hall Zwischenwasser & AT & Office & New & 1.45 & 0.67 \\
\hline 11 & Freihof Sulz & AT & Restaurant & Renovate & 2.14 & 1.86 \\
\hline 12 & Oeconomy building Josef Weiss & AT & Residential & New & 3.00 & 0.92 \\
\hline 13 & Giatla Haus & AT & Residential & Renovate & 3.04 & 0.89 \\
\hline
\end{tabular}




\begin{tabular}{|c|c|c|c|c|c|c|}
\hline 14 & Rhine Valley House Irgang & AT & Residential & Replace & 2.18 & 1.18 \\
\hline 15 & Baur Residence, Lustenau & AT & Residential & Replace & 2.70 & 0.64 \\
\hline 16 & Kasperhof & AT & Residential & Replace & 4.48 & 1.05 \\
\hline 17 & Maison Rubens & $\mathrm{BE}$ & Residential & Replace & & 1.04 \\
\hline 18 & $\begin{array}{l}\text { Half-timberframed house in Alken, } \\
\text { Belgium }\end{array}$ & $\mathrm{BE}$ & Residential & Replace & 4.70 & 1.27 \\
\hline 19 & Doragno Castle, Rovio (CH) & $\mathrm{CH}$ & Residential & New/replace & 4.40 & 0.84 \\
\hline 20 & Solar silo & $\mathrm{CH}$ & Office & New & 3.35 & 0.91 \\
\hline 21 & $\begin{array}{l}\text { Wohn- und Geschäftshaus } \\
\text { Feldbergstrasse }\end{array}$ & $\mathrm{CH}$ & Residential & Replace & 2.35 & 0.84 \\
\hline 22 & Mehrfamilienhaus Magnusstrasse & $\mathrm{CH}$ & Residential & & & \\
\hline 23 & St. Franziskus Church Ebmatingen & $\mathrm{CH}$ & Church & Renovate & 2.60 & 1.27 \\
\hline 24 & $\begin{array}{l}\text { Kindergarten and apartments } \\
\text { (PEB) Chur }\end{array}$ & $\mathrm{CH}$ & Educational & New & 3.00 & 0.80 \\
\hline 25 & $\begin{array}{l}\text { Single family home Luisenstrasse } \\
\text { - Bern }\end{array}$ & $\mathrm{CH}$ & Residential & New/renovate & 3.00 & 0.88 \\
\hline 26 & $\begin{array}{l}\text { Single Family House - Gstaad, } \\
\text { Switzerland }\end{array}$ & $\mathrm{CH}$ & Residential & Replace & 3.00 & 0.90 \\
\hline 27 & Glaserhaus in Affoltern & $\mathrm{CH}$ & Residential & Replace & 3.00 & 0.79 \\
\hline 28 & PalaCinema Locarno & $\mathrm{CH}$ & Culture & Replace & & 0.81 \\
\hline 29 & Casa Rossa Chemnitz & $\mathrm{DE}$ & Residential & Replace & 4.80 & 0.71 \\
\hline 30 & Rathaus Bergrheinfeld & $\mathrm{DE}$ & Town hall & Replace & & 1.12 \\
\hline 31 & Farmhouse Straub & $\mathrm{DE}$ & Residential & New & & 0.70 \\
\hline 32 & Early work Sep Ruf & $\mathrm{DE}$ & Residential & Replace & 2.31 & 0.98 \\
\hline 33 & Ackerbürgerhäuschen & $\mathrm{DE}$ & Residential & Replace & & 0.81 \\
\hline 34 & Ritterhof & $\mathrm{DE}$ & Residential & Replace & 4.62 & 0.60 \\
\hline 35 & Rathaus Burgkunstadt & $\mathrm{DE}$ & Town hall & Replace & 3.00 & 1.30 \\
\hline $36 a$ & Osramhuset (The Osram Building) & DK & Culture & Renovate & 5.90 & 1.20 \\
\hline $36 b$ & - Towards yard & - & & Replace & 2.70 & 1.20 \\
\hline $36 c$ & - Roof windows & - & & New & & 1.80 \\
\hline 37 & Klitgaarden & DK & Residential & Renovate & 5.80 & 0.70 \\
\hline 38 & Ryesgade 30 & DK & Residential & Replace & 4.61 & 1.04 \\
\hline 39 & $\begin{array}{l}\text { Timber-framed house in Alsace, } \\
\text { France }\end{array}$ & $\mathrm{FR}$ & Residential & Replace & 4.00 & 2.14 \\
\hline 40 & $\begin{array}{l}\text { Elementary School in Mulhouse, } \\
\text { France }\end{array}$ & FR & Educational & Replace & 2.80 & 1.40 \\
\hline 41 & $\begin{array}{l}\text { Timber-framed barn in the north of } \\
\text { France }\end{array}$ & $\mathrm{FR}$ & Residential & New & & 1.30 \\
\hline 42 & Rainhof & IT & Residential & Replace & 5.50 & 2.00 \\
\hline 43 & Villa Castelli & IT & Residential & Replace & 4.60 & 1.10 \\
\hline 44 & Ansitz Kofler & IT & Residential & Replace & 2.84 & 0.86 \\
\hline 45 & $\begin{array}{l}\text { Basilica di Santa Maria di } \\
\text { Collemaggio }\end{array}$ & $\mathrm{IT}$ & Church & & & \\
\hline 46 & House Pernter & IT & Residential & Replace & 5.56 & 1.31 \\
\hline 47 & Kohlerhaus & IT & Residential & Replace & 2.70 & 1.38 \\
\hline 48 & Ruckenzaunerhof & IT & Residential & Replace & 4.01 & 1.31 \\
\hline 49 & Aussergrubhof & IT & Residential & Replace & & 1.19 \\
\hline 50 & Oberbergerhof & IT & Residential & Replace & & 1.00 \\
\hline 51 & Platzbonhof & IT & Residential & Replace & 4.17 & 0.70 \\
\hline 52 & Mairhof & IT & Residential & Replace & 2.33 & 0.59 \\
\hline 53 & Obergasserhof & IT & Residential & Replace & 4.50 & 1.20 \\
\hline 54 & Rebecco Farm & IT & Residential & Replace & 4.96 & 1.46 \\
\hline 55 & Villa Capodivacca & $\mathrm{IT}$ & Residential & Replace & 2.67 & 1.28 \\
\hline 56 & House Moroder & IT & Residential & Replace & 1.79 & 0.82 \\
\hline 57 & Huberhof & IT & Residential & Replace & 4.18 & 1.18 \\
\hline 58 & Notarjeva vila & SL & Residential & Replace & 2.28 & 1.22 \\
\hline
\end{tabular}




\begin{tabular}{|l|l|l|l|l|l|l|}
\hline 59 & Rožna ulica 15, Idrija & SL & Residential & Replace & 5.80 & 1.10 \\
\hline 60 & Hiša trentarskih vodnikov & SL & Office & Replace & 5.00 & 1.17 \\
\hline 61 & Idrija mercury smelting plant & SL & Educational & Replace & 1.10 \\
\hline 62 & Mercado del Val, Valladolid (Spain) & SP & Wholesale/retail & Replace & 5.80 & 1.29 \\
\hline 63 & Correria 119 & SP & Residential & Replace & 1.73 & 1.10 \\
\hline 64 & Ahmet Aga Mansion & TR & Office & Renovate & 4.72 \\
\hline 65 & Necip Pasa Library & TR & Library & Replace & 4.99 & 2.76 \\
\hline 66 & Downie's Cottage & UK & Residential & Refurbish & 3.92 & 3.92 \\
\hline 67 & Hollyrood Park Lodge & UK & Wholesale/retail & Renovate & 4.48 & 1.26 \\
\hline 68 & Annat Road & UK & Residential & & & \\
\hline 69 & $\begin{array}{l}\text { Aspinall Courthouse - Net Zero } \\
\text { renovation }\end{array}$ & US & & & & \\
\hline
\end{tabular}

In table 12, "New" means that windows were added, i.e. in parts of the building where there were no windows before. "Replace" means that existing windows were replaced with either new windows or renovated windows where e.g. the frame was kept and the glazing replaced. "Refurbish" indicates that windows were renovated and re-fitted in the building without improvement of the energy aspects, and finally "Renovate" indicates that windows where renovated and the renovation included an improvement of the energy aspects. In e.g. Basilica di Santa Maria di Collemaggio no interventions were carried out for the windows. For Mehrfamilienhaus Magnusstrasse, Annat Road and Mariahilferstrasse no information was available on the window solutions.

In most cases, there is a significant improvement of the energy aspects of the windows. Only in two cases there was no energy improvement, i.e. in Downie's Cottage and Hof 6, Schwarzenberg, Vorarlberg, Austria. In Downie's Cottage windows were few and very small, therefore it was deemed unnecessary to improve the windows, and instead they were simply carefully renovated.

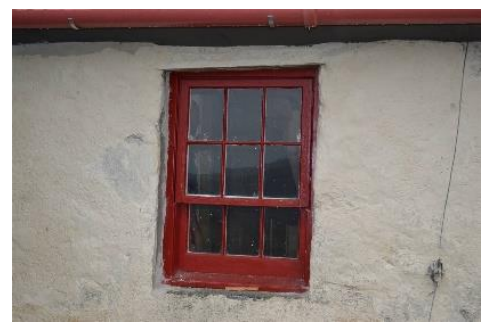

Figure 24: Downie's Cottege. Few and very small windows.

Figure 24 shows a comparison of the approximate total window $U$-values before and after the renovation. From the figure it is quite clear that in most cases, the renovation has increased the energy efficiency of the windows significantly.

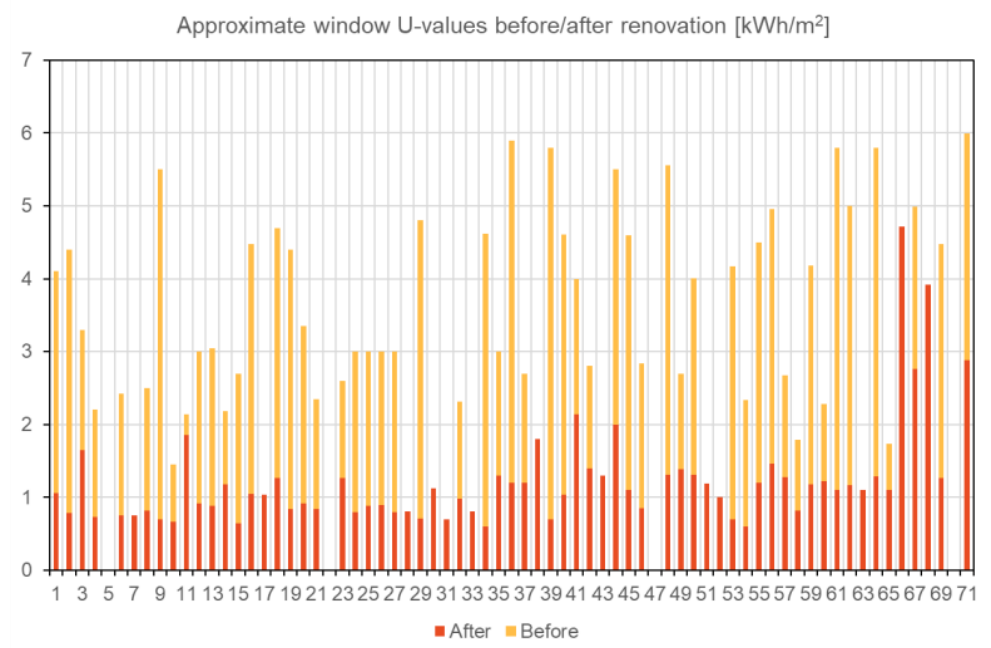

Figure 25: Window U-values before and after renovation. 
In buildings where for some reason it is not possible or does not make sense to replace or improve the windows, a careful renovation can restore the original windows to increase their lifetime and improve their appearance.

The main reason for the improvement in the rest of the cases are due to the fact that single-layer glazing is replaced by either double- or triple-layer glazing, however, some cases also see slight improvements in window frames, reveals etc.

\subsubsection{Adding windows}

There are 10 cases where new windows were added to the buildings, i.e. installing windows in parts of the building that did not have windows before the renovation. Common to these solutions are that the new windows are highly energy efficient, since there were no restrictions.

Solar Silo used to be a coal silo and heating plant and it was transformed into an office building. Therefore, the building had only very few windows to start with and it was necessary to add windows to supply adequate daylighting for the new use. This also meant that there were no restrictions on the window solutions and therefore highly efficient low energy windows with 3-layers of glass, argon filling, a wooden frame and a mean U-value of approx. $1.0 \mathrm{~W} / \mathrm{m}^{2} \mathrm{~K}$ could be chosen.

In Osramhuset new roof windows were added. Only the building's façade was protected and therefore adding roof windows was a way to significantly increase daylighting levels in the building while also providing an opportunity for natural ventilation. The roof windows were double-layer wood/aluminium windows with argon and a U-value of $1.80 \mathrm{~W} / \mathrm{m}^{2} \mathrm{~K}$.

In Doragno Castle new large windows and glass facades were added. The original stone walls are highlighted by the large windows completing the volume of the existing building. The windows have double- or triple-layer glass and aluminium frame and the $U$-value ranges from $1.0-1.2 \mathrm{~W} / \mathrm{m}^{2} \mathrm{~K}$.

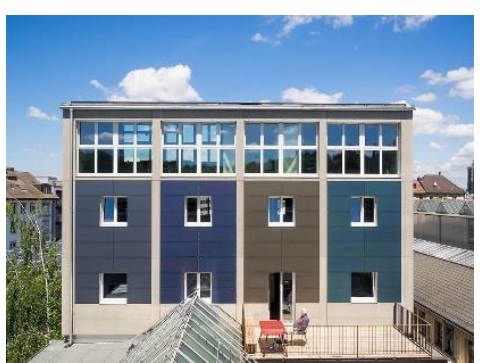

Figure 26: Solar silo. New windows added to the façade.

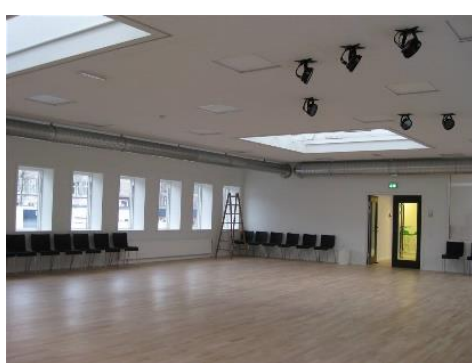

Figure 27: Osramhuset. New roof windows increased daylighting levels.

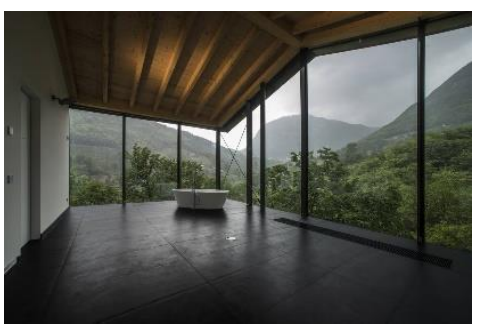

Figure 28: Doragno Castle. New large windows to improve daylighting.

Adding new windows is a way to achieve a high level of energy efficiency and improving daylighting in the buildings, however, this solution will only be relevant in buildings or parts of buildings that are not protected and where there is a high degree of freedom for the architect. In the Solar Silo it was necessary to transform the building from silo to offices and hence add windows; in Osramhuset roof windows did not change the protected façade and improved daylighting levels significantly and in Doragno Castle there were no restrictions which allowed for adding a modern touch to the building with panoramic windows.

\subsubsection{Replacing windows}

In 45 of the analysed solutions, the existing windows were replaced with new ones. In eight of these, there were no restrictions regarding the new windows and therefore off-the-shelf solutions with no specific appearance could be used, e.g. in Osramhuset it was the windows facing the back of the building and in Doragno Castle there were no restrictions in general.

For Villa Castelli, the original windows were not of heritage value and therefore they were replaced with new more energy-efficient windows. The new windows were handcrafted by a carpenter in wood-aluminium and the only requirement of the heritage authority was to maintain the original layout of the windows (number and thickness of sashes etc.). 


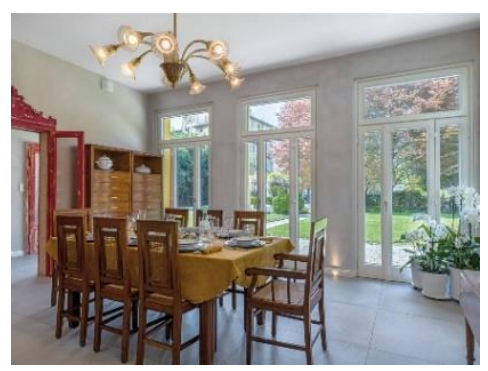

Figure 29: Villa Castelli. Old windows replaced by similar new ones.

In 13 cases, e.g. Mairhof, Obergasserhof, Platzbonhof, Aussergrubhof, Ruckenzaunerhof and Mercado del Val, replaced the original windows with new more or less off-the-shelf solutions, i.e. no specialized craftsmanship was necessary to produce the windows. This also meant that there were no particular focus on heritage values and the windows were chosen to have similar characteristics and outlook as the original windows. The energy improvement is therefore also significant and U-values are typically reduced by $70-80 \%$.

In Mairhof, all windows and doors, including existing PVC windows with double-glazing, were replaced by wooden windows with triple glazing.

For Obergasserhof, as part of the energy upgrade, the old windows from the 1970s were replaced with new, single-leaf and double-glazed lattice windows.

In Platzbonhof, the existing windows were replaced by new triple-glazed wooden windows. The windows have been completely replaced, only the lintel has remained.

Aussergrubhof had existing windows from the 1970s. These windows were replaced by larch wood windows with double-glazing.

In Ruckenzaunerhof, indoor lighting levels was a problem due to the small-format windows. To ensure that enough light enters the house, clear views were an important point during the renovation. This also increased the solar energy gains. All existing windows were replaced with new double-glazed windows in order to meet today's energy standards. The selection of new windows was carried out in close cooperation with the monument office. The original materiality of the wooden windows, as well as the typical rungs should definitely be restored.

In Mercado del Val a new glazed façade was added and existing windows were replaced to allow for natural ventilation. The glazing façade increases daylighting and makes the commercial activities visible from the outside. The original windows and skylights were not catalogued as a heritage part of the building. The overall U-value of windows and glass façade was improved by approx. $75 \%$.

The following figures show examples from the six buildings.

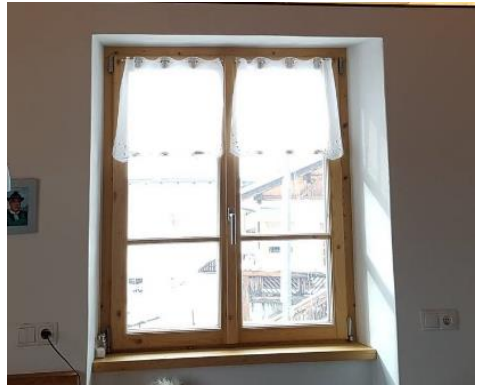

Figure 30: Mairhof. All windows were replaced.

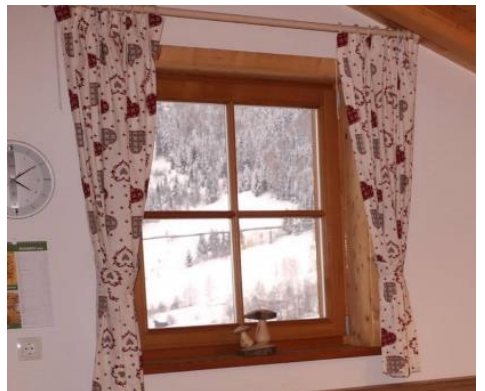

Figure 31: Obergasserhof. Singleleaf and double-glazed new windows.

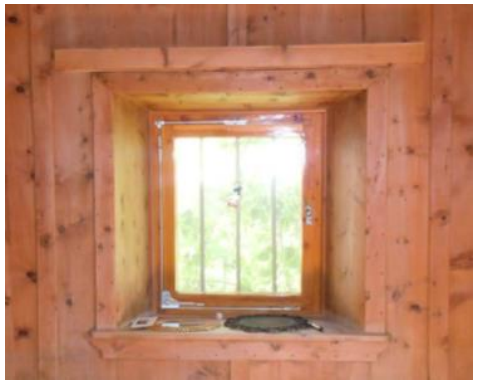

Figure 32: Platzbonhof. Tripleglazed windows improved energy efficiency. 


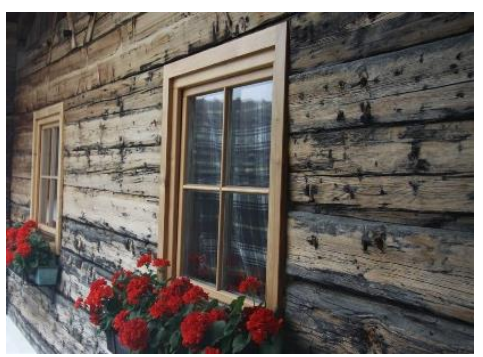

Figure 33: Aussergrubhof. Larch wood double-glazed windows.

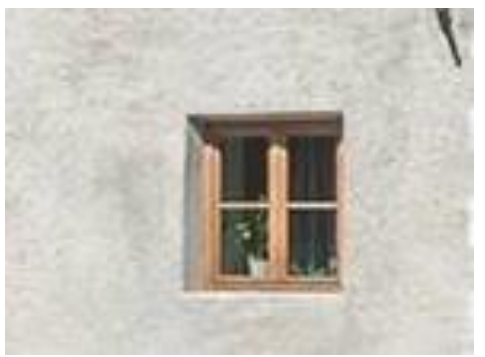

Figure 34: Ruckenzaunerhof

New windows selected in close

cooperation with monument office.

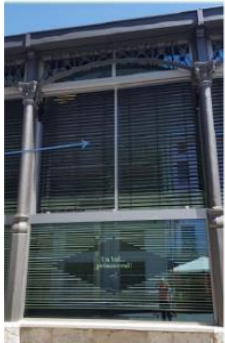

Figure 35: Mercado del Val. The new glazed façade increased daylighting levels.

In the remaining 24 solutions, e.g. Ansitz Kofler, Bauernhof Trins, Maison Rubens, Half-timberframed house in Alken, Oberbergerhof, Timber-Framed house in Alsace and Rainhof all new windows were handcrafted to match the originals as far as possible. This meant typically that the frame would be an exact replica of the original except for the fact that it was adjusted to allow for another type of glazing, i.e. double- or triple-layer low energy glazing instead of the typical single-layer glazing. The change of the glazing reduces heat losses significantly making the windows perform more or less like today's standard.

In the west façade of Ansitz Kofler, where the wall was insulated from the outside, the ancient openings were reconstructed. In order to allow for the most efficient position of the new window in a line with the insulation layer, the sub-frame had to be placed just "outside" the original stonewall. In order to avoid thermal bridges, the rollershutter casings were installed on the wall in the insulation layer and the sub-frame was completely covered by the mineral wool panels. In order to achieve an airtight connection between sub-frame and the main frame of the window, jointing tape was applied.

In Bauernhof Trins, the windows were completely replaced. The new wooden windows in the local style were pre-installed in the custom-made wooden wall elements. The optimized position of the window frames in the insulation layer increases thermal comfort and reduces the risk of condensation through minimized thermal bridges.

For Maison Rubens, the windows were replaced with new ones of oak wood. The new windows are identical to the original and has the original shape. Most of the windows have been replaced and only the ones on the front façade at first floor were kept because they already had double-glazing.

In the Half-timberframed house in Alken, the windows were in a very bad state. There were windows (remains) from the 17th, 19th and even 20th century. In some places the windows were also missing. The remaining wooden joinery in the half-timbered house has been completely replaced by typologically appropriate new joinery. The new windows have double-glazing and in order to accommodate this, the profile thickness has been adjusted slightly.

In Oberbergerhof, most of the windows have been replaced. The bifora windows (mullioned windows with two lights) from the late renaissance were preserved in the middle room on the second floor. The windows in the apartment were renewed, while four new windows were added to the roof on the top floor. In the knight's hall, the old, historical windows have been preserved and not changed. When replacing the windows, special care was taken to ensure that the view of the courtyard was not changed. Therefore, it was only possible to provide the very thin frames, which appear historic in shape, with double-layer glazing, i.e. triple-layer glazing was not an option.

In Timber-framed house in Alsace all the original single-glazed wooden windows were replaced by doubleglazed ones dedicated especially to heritage buildings. Thanks to pictures from the beginning of the 20th century, it was possible to restore the windows as they were originally. The windows were made to measure by a local joiner. Heritage authorities had to be convinced that a double-glazed solution would fit the original design of the windows and therefore the owner and the joiner had to produce a prototype of the window which that finally convinced the heritage board.

In Rainhof all existing windows were replaced with new units made ad-hoc by a local furniture maker. The aim was building a two-sash window with two glazing bars each that on the one hand achieved a high energy performance and on the other hand were of high aesthetic quality. One of the original windows was used as a 
model for the new windows in terms of proportions and profile widths. To improve the thermal performance of the window, a double-glazed unit was installed and this improves the U-value by approx. $60 \%$.

In Baur Residence, Lustenau, the windows from the 1960s were replaced and building sins were eradicated by partially moving the windows back to their original place. This not only restored the character of the Rhinevalley house, but also brought more daylight into the building. The existing windows on the upper floors were replaced by windows in passive house quality.

In Single family home Luissenstrasse - Bern, most of the existing windows have been renovated and only a few have been replaced with a copy, due to the bad state of conservation.

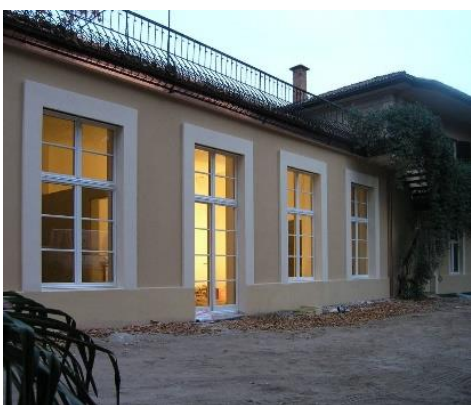

Figure 36: Ansitz Kofler. Façade restored to original state.

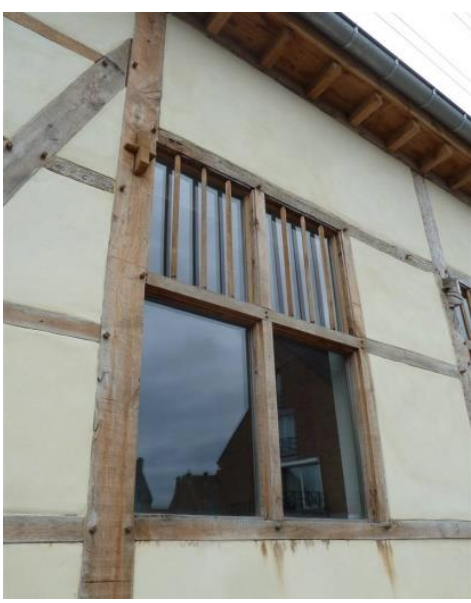

Figure 39: Half-timberframed house in Alken. Handcrafted windows.

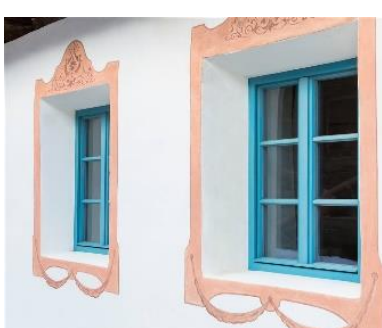

Figure 42: Rainhof. Windows produced by local furniture maker.

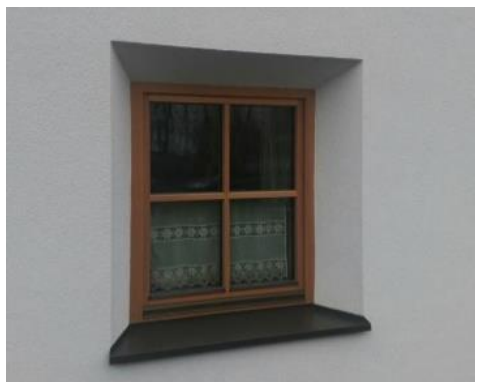

Figure 37: Bauernhof Trins. Windows is local style.

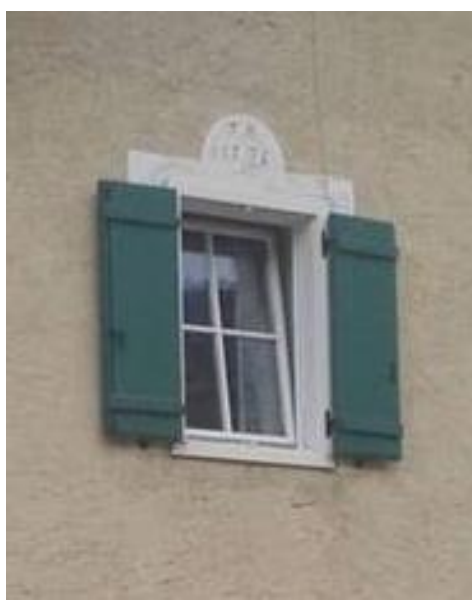

Figure 40: Oberbergerhof. Thin frame with a historic appearance.

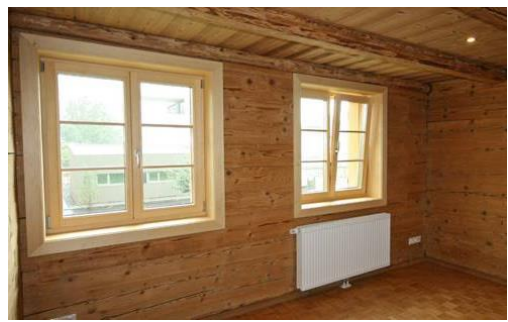

Figure 43: Baur Residence,

Lustenau. Windows moved back to original place

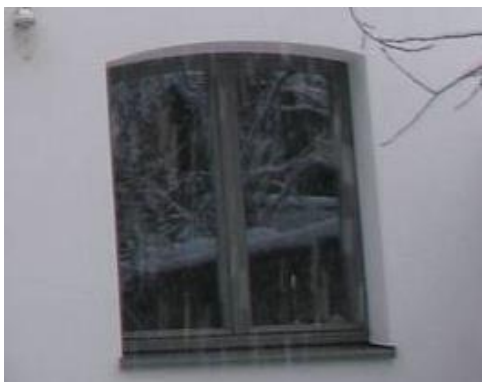

Figure 38: Maison Rubens. New oak wood windows.

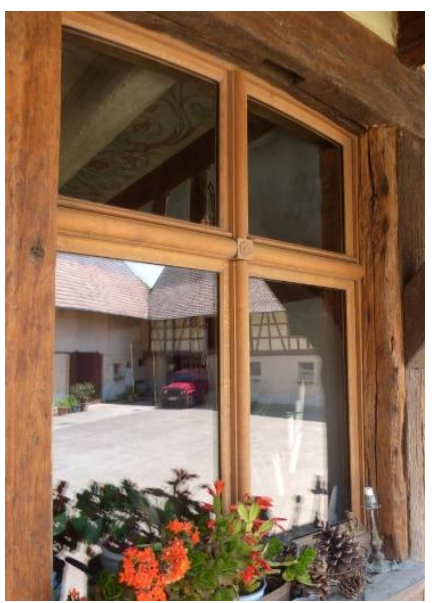

Figure 41: Timber-framed house in Alsace. New double-glazed solution.

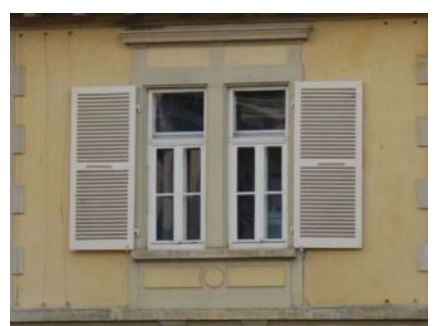

Figure 44: Single family home

Luisenstrasse - Bern. Most of the existing windows have been renovated

From the figures above it is quite clear that windows are new, however, the workmanship is clearly professional and the windows fit perfectly in the renovated building. Even though the windows match perfectly the original style of the buildings, the overall U-value is significantly improved. Typically, U-value is reduced from $3.0-5.0 \mathrm{~W} / \mathrm{m}^{2} \mathrm{~K}$ to $1.0 \mathrm{~W} / \mathrm{m}^{2} \mathrm{~K}$, primarily due to the fact that glazing has been changed from single-layer or old double-layer to either low energy double-layer or even triple-layer. 


\subsubsection{Renovating windows}

In the buildings where replacing the windows was not an option, focus has instead been on restoring and renovating the existing windows. In 11 cases, the windows were renovated in a way which also improved the energy efficiency, e-g- Osramhuset, Hollyrood Park Lodge, Klitgaarden and Klostergebäude Kaiserstrasse.

For Osramhuset the original façade could not be changed in any way and therefore improving the energy efficiency had to be done on the inside. On the inside of the façade walls a sheet of glass was added that spanned from floor to ceiling, i.e. covering both the walls themselves and the windows. From the outside this is not visible, but it has a significant influence on the overall $U$-value of the windows that is reduced from approx. $5.90 \mathrm{~W} / \mathrm{m}^{2} \mathrm{~K}$ to $1.20 \mathrm{~W} / \mathrm{m}^{2} \mathrm{~K}$ and also on the indoor climate where it helps remove draughts.

In Hollyrood Park Lodge the frame of the existing windows was in good condition and therefore the decision was made to keep them. The glazing was replaced by new slim-profile double-glazed units, which significantly improved the energy characteristics of the windows without changing notably the appearance. The $U$-value is reduced by approx. $70 \%$ while the new glazing also improves the indoor climate by removing draughts.

In Klitgaarden the old wooden windows have been renovated and reused. The glazing sash bar has been partially removed making it possible to replace three smaller panes with one larger. No information is available on the overall U-value, but the glazing has improved from $5.80 \mathrm{~W} / \mathrm{m}^{2} \mathrm{~K}$ to $0.85 \mathrm{~W} / \mathrm{m}^{2} \mathrm{~K}$.

In Klostergebäude Kaisterstrasse the outer part of the box-windows in listed facades were renovated and, on the inside, a new wooden window with special interior insulation was added. The new inner wing is completely flush with the inner wall and reduces thermal bridges through internal insulation and reveal insulation. The shading is positioned between the wings in the lintel in existing roller blind niches. The new solution reduces the heat loss through windows by approx. $75 \%$.

On the ground floor of Freihof Sulz, where possible, the well-preserved cambered windows are retained. These are box-type windows with curved panes on the outside and normal single glazing on the inside.

In Giatla House new larch windows were installed on the inside, behind the historical windows.

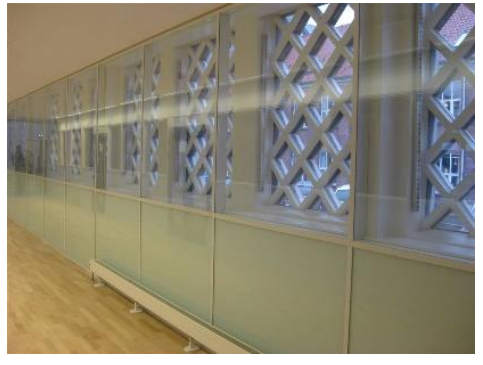

Figure 45: Osramhuset.

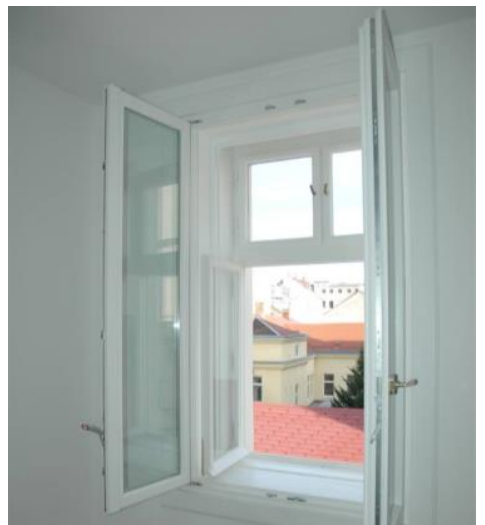

Figure 48: Klostergebäude Kaiserstrasse.

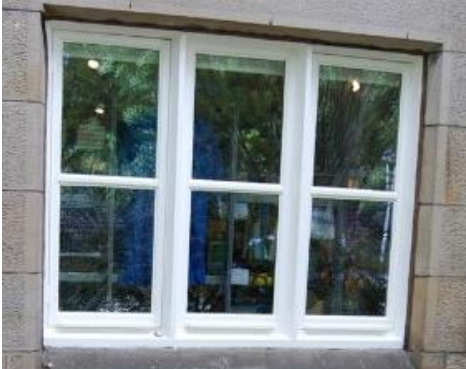

Figure 46: Hollyrood Park Lodge.

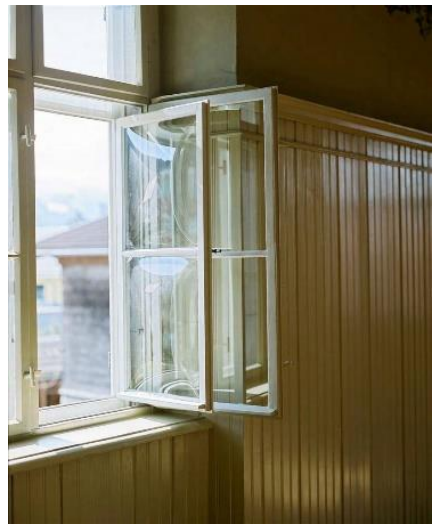

Figure 49: Freihof Sulz. (Source: Lukas Schaller)

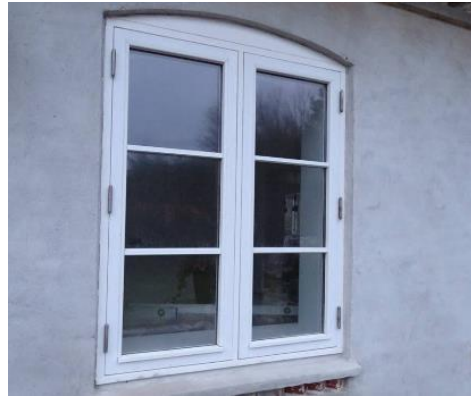

Figure 47: Klitgaarden.

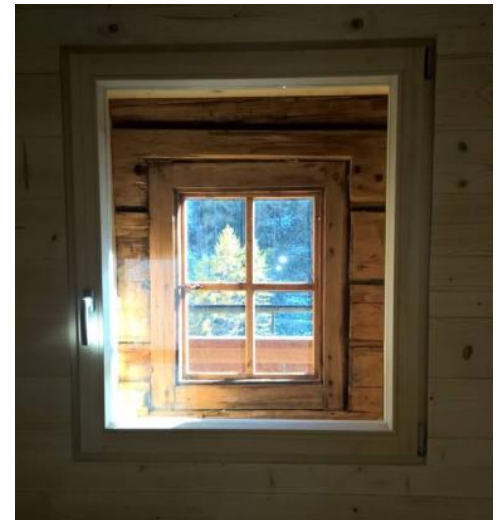

Figure 50: Giatla House. (Source: Benjamin Schaller)

When e.g. the façade of the building is protected it is often not possible to change or replace the windows. Instead attention can be turned towards the inside part, where adding extra layers of glass can have a significant effect on heat losses. The solutions shown in the figures above are very good examples showing how energy efficiency and preserving heritage values can go hand-in-hand. 


\subsection{HVAC}

\subsubsection{Heating}

\subsubsection{Overview of heating}

In 62 out of the 69 studied cases a change in the heating system was documented. With 25 buildings more than one third changed to a biomass boiler system - be it pellets, wood chip or wooden boilers. 7 buildings are connected to district heating and considering that 4 of those are based on biomass the total number of buildings with biomass as main source for heating sums up to 29.18 buildings are now equipped with heat pumps, 2 of them explicitly mentioning, that the electricity is derived from on-site PV. The major part of heat pump system is connect to a geothermal heat source, but also air as heat source is common. 9 buildings have gas heating systems, and only one has solar thermal as main heating system.

- 25 case studies heated with any kind of biomass

- 7 cases studied with district heating (4 of which based on biomass, two at least partly on waste)

- 18 case studies with heat pumps (2 of which explicitly with electricity from PV, half of the systems with geothermal heat source, one third with air and the rest without explicit information)

- 9 case studies with gas

- 1 case study is heated still with oil

- 1 case study is heated with solar thermal panels

- 1 case study has a direct electric system

- 1 case study has no heating system

- 1 case mentions that the heating system is not known and

- 5 do not have any information.

The tendency towards biomass and heat pump systems is especially pronounced in rural residential buildings, as Figure 51 shows. Gas condensing boilers have been chosen mainly in urban residential buildings. Other systems which include the district heating systems (7 out of 10 ) are most often use in not residential buildings.
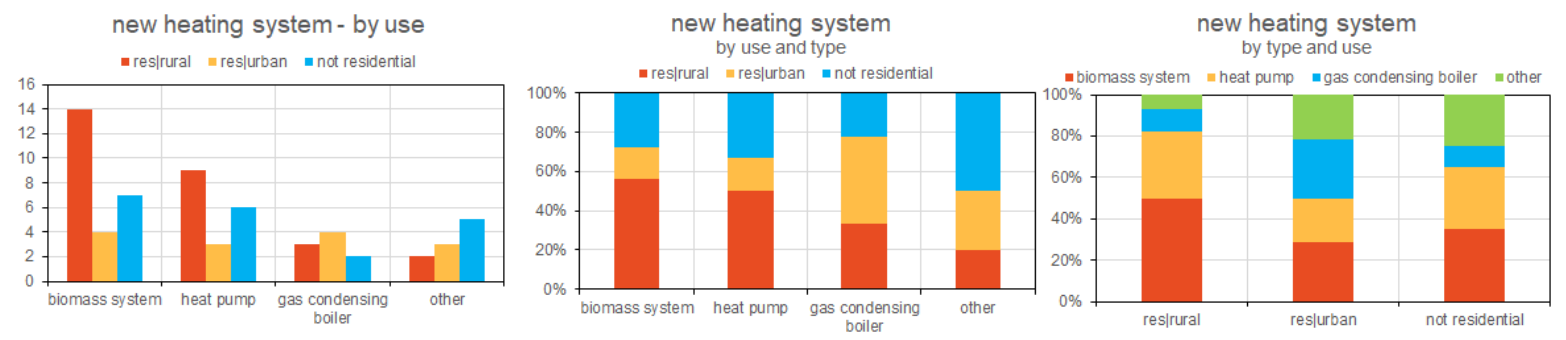

Figure 51: New heating system by use and system type

In a bit more than half of the cases (39 out of 69) information was also given on the old system which was exchanged (including the 6 buildings with explicitly say, that the building had not been heated). The change of system has been depicted in the left sankey diagram with redish colours for not renewable and yellowish colours for renewable energy systems (district heating and heat pump remaining grey, as they can be both): there is clearly a shift from not renewable to renewable systems visible, even if the completely new systems were in one third of the cases not renewable ones.

Solar thermal systems come mainly into use as secondary system, together with biomass systems in the majority of cases but not only. The right sankey diagram points out the solar system (with yellowish link), but it also gives the complete overview of all 31 buildings for which a secondary heating system has been documented: here even more renewable energy systems dominate, with 8 cases involving solar systems and 10 cases involving wood fired stoves.

Interesting to observe is also that in $\mathbf{1 8}$ cases, thus more than one fourth of documented cases, the original wood stove was kept in use, in most cases as secondary heating system: Thereof 15 cases are rural residential buildings (out of 31 , i.e. $50 \%$ ), 2 are hotels (out of 4 , so again $50 \%$ ) and 1 is a urban residential building (out of 17). 4 "kept stoves" are documented in Austria (making up $25 \%$ of the documented cases), 1 in Switzerland (10\% of cases), 2 in Germany (30\% of cases), 1 in France (33\%), 1 in the United Kingdom (again 33\%) and actually 9 
in Italy (corresponding to more than $50 \%$ of the documented cases) where this high number corresponds however to a high share of rural residential buildings having been documented.

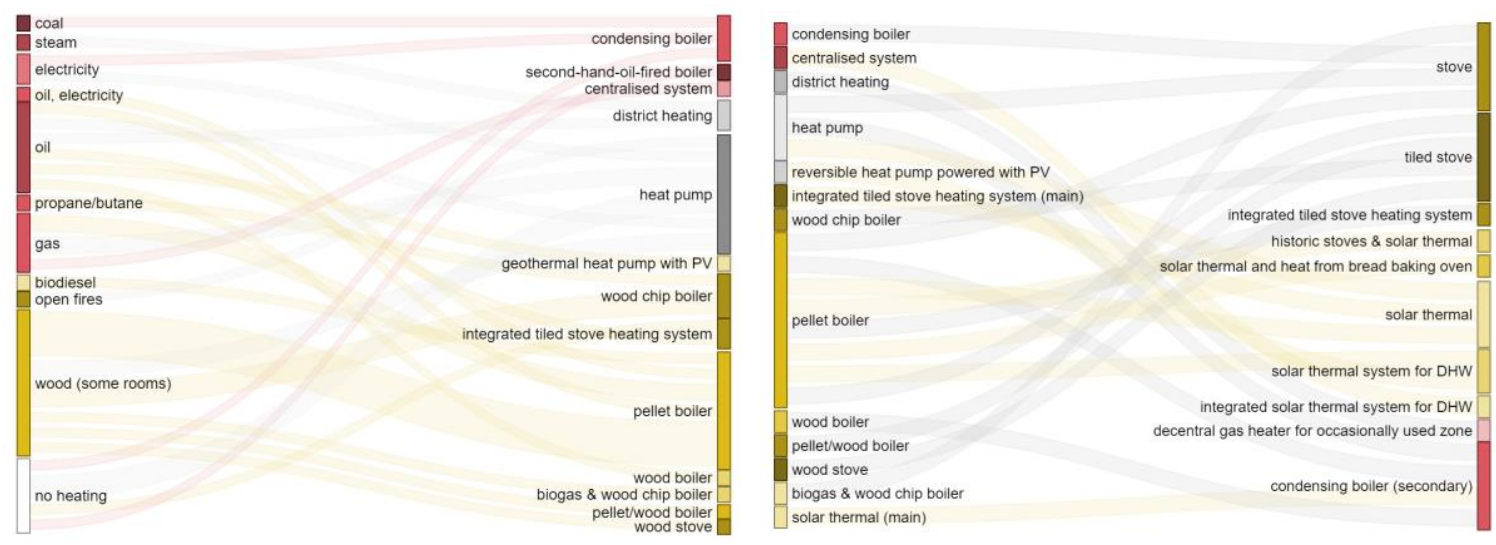

Figure 52: Change of heating system (for the 39 cases where this information is available)

Table 13: Overview of heating

\begin{tabular}{|c|c|c|c|c|c|c|c|c|}
\hline No & Case study & $\begin{array}{l}\text { Cou } \\
\text { ntry }\end{array}$ & $\begin{array}{l}\text { Old } \\
\text { system }\end{array}$ & $\begin{array}{l}\text { New main } \\
\text { system } \\
\text { type }\end{array}$ & $\mathbf{k W}$ & fuel & $\begin{array}{l}\text { Distribution } \\
\text { system }\end{array}$ & $\begin{array}{l}\text { Secon-dary } \\
\text { system }\end{array}$ \\
\hline 1 & $\begin{array}{l}\text { Klostergebäude } \\
\text { Kaiserstrasse }\end{array}$ & AT & & district heating & 160 & $\begin{array}{l}\text { waste and } \\
\text { CHP }\end{array}$ & $\begin{array}{l}\text { floor heating, } \\
\text { radiators or wall } \\
\text { heating }\end{array}$ & \\
\hline 2 & Farm house Trins & AT & & heat pump & 12.3 & geothermal & radiating floor & old stoves \\
\hline 3 & $\begin{array}{l}\text { Hof } 6 \text {, Schwarzenberg, } \\
\text { Voralberg, Austria }\end{array}$ & AT & oil & pellet boiler & & biomass & radiators & $\begin{array}{l}\text { solar thermal } \\
\text { system for } \\
\text { DHW }\end{array}$ \\
\hline 4 & $\begin{array}{l}\text { Kelchalm - Bochumer } \\
\text { alpine hut }\end{array}$ & AT & biodiesel & pellet boiler & 22 & biomass & radiators & $\begin{array}{l}\text { solar thermal } \\
\text { system for } \\
\text { DHW }\end{array}$ \\
\hline 5 & Mariahilferstrasse 182 & AT & gas & $\begin{array}{l}\text { centralised } \\
\text { system }\end{array}$ & 150 & gas & radiating floor & $\begin{array}{l}\text { integrated } \\
\text { solar thermal } \\
\text { system for } \\
\text { DHW }\end{array}$ \\
\hline 6 & House Maurer, Wolfurt & AT & & & & & & \\
\hline 7 & $\begin{array}{l}\text { House Breuer, } \\
\text { Tschagguns }\end{array}$ & AT & $\begin{array}{l}\text { no } \\
\text { heating }\end{array}$ & $\begin{array}{l}\text { integrated tiled } \\
\text { stove heating } \\
\text { system }\end{array}$ & & biomass & $\begin{array}{l}\text { radiating floor } \\
\text { and wall }\end{array}$ & solar thermal \\
\hline 8 & Music school in Velden & AT & & district heating & 42 & $\begin{array}{l}\text { Ind. heat, } \\
\text { biomass } \\
\text { and gas }\end{array}$ & $\begin{array}{l}\text { radiating floor, } \\
\text { radiators and air }\end{array}$ & \\
\hline 9 & Hof Neuhäusl & AT & & heat pump & 7 & $\begin{array}{l}\text { air and } \\
\text { electricity }\end{array}$ & radiating floor & \\
\hline 10 & $\begin{array}{l}\text { Community Hall } \\
\text { Zwischenwasser }\end{array}$ & AT & & district heating & & biomass & radiating floor & \\
\hline 11 & Freihof Sulz & AT & $\begin{array}{l}\text { tiled } \\
\text { stoves }\end{array}$ & pellet boiler & 110 & biomass & radiating ceiling & $\begin{array}{l}\text { solar thermal } \\
\text { and heat from } \\
\text { bread baking } \\
\text { oven }\end{array}$ \\
\hline 12 & $\begin{array}{l}\text { Oeconomy building } \\
\text { Josef Weiss }\end{array}$ & AT & $\begin{array}{l}\text { no } \\
\text { heating }\end{array}$ & $\begin{array}{l}\text { condensing } \\
\text { boiler }\end{array}$ & 12 & gas & $\begin{array}{l}\text { partly concrete } \\
\text { core activation }\end{array}$ & \\
\hline 13 & Giatla Haus & AT & stoves & pellet boiler & 21.8 & biomass & radiating floor & \\
\hline
\end{tabular}




\begin{tabular}{|c|c|c|c|c|c|c|c|c|}
\hline 14 & $\begin{array}{l}\text { Rhine Valley House } \\
\text { Irgang }\end{array}$ & AT & oil & $\begin{array}{l}\text { integrated tiled } \\
\text { stove heating } \\
\text { system }\end{array}$ & 15 & biomass & $\begin{array}{l}\text { radiators and } \\
\text { wall heating } \\
\text { System }\end{array}$ & \\
\hline 15 & $\begin{array}{l}\text { Baur Residence, } \\
\text { Lustenau }\end{array}$ & AT & $\begin{array}{l}\text { oil, elec- } \\
\text { tricity }\end{array}$ & pellet boiler & 8.5 & pellets & radiators & tiled stove \\
\hline 16 & Kasperhof & AT & & $\begin{array}{l}\text { condensing } \\
\text { boiler }\end{array}$ & 36.9 & gas & radiating floor & \\
\hline 17 & Maison Rubens & $\mathrm{BE}$ & & $\begin{array}{l}\text { condensing } \\
\text { boiler }\end{array}$ & 17.2 & gas & radiators & \\
\hline 18 & $\begin{array}{l}\text { Half-timberframed house } \\
\text { in Alken, Belgium }\end{array}$ & $\mathrm{BE}$ & & $\begin{array}{l}\text { condensing } \\
\text { boiler }\end{array}$ & 35 & gas & radiating floor & \\
\hline 19 & $\begin{array}{l}\text { Doragno Castle, Rovio } \\
\text { (CH) }\end{array}$ & $\mathrm{CH}$ & & $\begin{array}{l}\text { reversible heat } \\
\text { pump powered } \\
\text { with PV }\end{array}$ & 44.8 & air & radiating floor & $\begin{array}{l}\text { solar thermal } \\
\text { system for } \\
\text { DHW }\end{array}$ \\
\hline 20 & Solar silo & $\mathrm{CH}$ & & district heating & & $\begin{array}{l}\text { waste } \\
\text { incinerator }\end{array}$ & radiators & $\begin{array}{l}\text { decentral gas } \\
\text { heater for } \\
\text { occa-sionnally } \\
\text { used zone }\end{array}$ \\
\hline 21 & $\begin{array}{l}\text { Wohn- und } \\
\text { Geschäftshaus } \\
\text { Feldbergstrasse }\end{array}$ & $\mathrm{CH}$ & & & 13 & solar & radiators & \\
\hline 22 & $\begin{array}{l}\text { Mehrfamilienhaus } \\
\text { Magnusstrasse }\end{array}$ & $\mathrm{CH}$ & & pellet boiler & 32 & biomass & radiators & \\
\hline 23 & $\begin{array}{l}\text { St. Franziskus Church } \\
\text { Ebmatingen }\end{array}$ & $\mathrm{CH}$ & oil & heat pump & 20 & geothermal & $\begin{array}{l}\text { partly radiator, } \\
\text { partly } \\
\text { floorheating }\end{array}$ & solar thermal \\
\hline 24 & $\begin{array}{l}\text { Kindergarten and } \\
\text { apartments (PEB) Chur }\end{array}$ & $\mathrm{CH}$ & gas & pellet boiler & 22 & pellets & radiators & solar thermal \\
\hline 25 & $\begin{array}{l}\text { Single family home } \\
\text { Luisenstrasse - Bern }\end{array}$ & $\mathrm{CH}$ & gas & heat pump & 8 & geothermal & $\begin{array}{l}\text { Radiators and } \\
\text { radiators wall }\end{array}$ & solar thermal \\
\hline 26 & $\begin{array}{l}\text { Single Family House - } \\
\text { Gstaad, Switzerland }\end{array}$ & $\mathrm{CH}$ & $\begin{array}{l}\text { no } \\
\text { heating }\end{array}$ & heat pump & 6 & air & radiating floor & \\
\hline 27 & Glaserhaus in Affoltern & $\mathrm{CH}$ & & heat pump & 17 & geothermal & radiating floor & $\begin{array}{l}\text { swiss stop } \\
\text { stove }\end{array}$ \\
\hline 28 & PalaCinema Locarno & $\mathrm{CH}$ & & heat pump & 450 & geothermal & & \\
\hline 29 & Casa Rossa Chemnitz & $\mathrm{DE}$ & & solar thermal & 18 & solar & radiating floor & $\begin{array}{l}\text { condensing } \\
\text { boiler }\end{array}$ \\
\hline 30 & Rathaus Bergrheinfeld & $\mathrm{DE}$ & & pellet boiler & & biomass & radiators & $\begin{array}{l}\text { condensing } \\
\text { boiler }\end{array}$ \\
\hline 31 & Farmhouse Straub & $\mathrm{DE}$ & & pellet boiler & 18 & biomass & radiating wall & $\begin{array}{l}\text { historic stoves } \\
\text { \& solar }\end{array}$ \\
\hline 32 & Early work Sep Ruf & $\mathrm{DE}$ & oil & district heating & & biomass & radiators & \\
\hline 33 & Ackerbürgerhäuschen & $\mathrm{DE}$ & coal & $\begin{array}{l}\text { condensing } \\
\text { boiler }\end{array}$ & 14.2 & gas & radiating floor & stove \\
\hline 34 & Ritterhof & $\mathrm{DE}$ & & pellet boiler & 32 & pellets & radiating wall & $\begin{array}{l}\text { integrated tiled } \\
\text { stove heating } \\
\text { system }\end{array}$ \\
\hline 35 & Rathaus Burgkunstadt & $\mathrm{DE}$ & $\begin{array}{l}\text { electricit } \\
\mathrm{y}\end{array}$ & $\begin{array}{l}\text { condensing } \\
\text { boiler }\end{array}$ & 45 & gas & radiating wall & \\
\hline 36 & $\begin{array}{l}\text { Osramhuset (The Osram } \\
\text { Building) }\end{array}$ & DK & steam & district heating & & & radiators & \\
\hline 37 & - Towards yard & DK & electricit & heat pump & 10 & & floor heating & \\
\hline 38 & - Roof windows & DK & & & & & & \\
\hline 39 & Klitgaarden & FR & & pellet boiler & 25 & biomass & $\begin{array}{l}\text { radiating } \\
\text { baseboards }\end{array}$ & stove \\
\hline 40 & Ryesgade 30 & FR & & $\begin{array}{l}\text { condensing } \\
\text { boiler }\end{array}$ & & gas & $\begin{array}{l}\text { radiators \& floor } \\
\text { heating }\end{array}$ & \\
\hline 41 & $\begin{array}{l}\text { Timber-framed house in } \\
\text { Alsace, France }\end{array}$ & FR & $\begin{array}{l}\text { no } \\
\text { heating }\end{array}$ & $\begin{array}{l}\text { second-hand- } \\
\text { oil-fired boiler }\end{array}$ & & oil & $\begin{array}{l}\text { floor heating and } \\
\text { old stove } \\
\text { operated from } \\
\text { central heating }\end{array}$ & \\
\hline
\end{tabular}




\begin{tabular}{|c|c|c|c|c|c|c|c|c|}
\hline 42 & $\begin{array}{l}\text { Elementary School in } \\
\text { Mulhouse, France }\end{array}$ & IT & & $\begin{array}{l}\text { biogas \& wood } \\
\text { chipp boiler }\end{array}$ & & biomass & radiating floor & stove \\
\hline 43 & $\begin{array}{l}\text { Timber-framed barn in } \\
\text { the north of France }\end{array}$ & IT & oil & $\begin{array}{l}\text { geothermal } \\
\text { heat pump } \\
\text { with PV }\end{array}$ & & geothermal & radiating floor & \\
\hline 44 & Rainhof & IT & gas & pellet boiler & 14 & biomass & radiating floor & tiled stove \\
\hline 45 & Villa Castelli & IT & & heat pump & & geothermal & $\begin{array}{l}\text { hydronic high- } \\
\text { efficiency pew- } \\
\text { based system }\end{array}$ & \\
\hline 46 & Ansitz Kofler & IT & & pellet boiler & 30 & biomass & radiating floor & stove \\
\hline 47 & $\begin{array}{l}\text { Basilica di Santa Maria } \\
\text { di Collemaggio }\end{array}$ & IT & & district heating & 14.9 & biomass & $\begin{array}{l}\text { combination of } \\
\text { wall heating } \\
\text { (ground floor) } \\
\text { and radiators } \\
\text { (other floors) }\end{array}$ & \\
\hline 48 & House Pernter & IT & & & & not known & $\begin{array}{l}\text { floor and wall } \\
\text { heating }\end{array}$ & tiled stove \\
\hline 49 & Kohlerhaus & IT & $\begin{array}{l}\text { only part } \\
\text { with } \\
\text { wood }\end{array}$ & $\begin{array}{l}\text { wood chip } \\
\text { boiler }\end{array}$ & & biomass & $\begin{array}{l}\text { radiator \& floor } \\
\text { heating }\end{array}$ & tiled stove \\
\hline 50 & Ruckenzaunerhof & IT & $\begin{array}{l}\text { only part } \\
\text { with } \\
\text { wood }\end{array}$ & $\begin{array}{l}\text { pellet/wood } \\
\text { boiler }\end{array}$ & & $\begin{array}{l}\text { Wood and } \\
\text { Pellets }\end{array}$ & radiating floor & tiled stove \\
\hline 51 & Aussergrubhof & IT & $\begin{array}{l}\text { only part } \\
\text { with } \\
\text { wood }\end{array}$ & $\begin{array}{l}\text { wood chip } \\
\text { boiler }\end{array}$ & & biomass & radiators & tiled stove \\
\hline 52 & Oberbergerhof & IT & oil & wood boiler & 30 & biomass & radiating floor & $\begin{array}{l}\text { condensing } \\
\text { boiler }\end{array}$ \\
\hline 53 & Platzbonhof & IT & $\begin{array}{l}\text { only part } \\
\text { with } \\
\text { wood }\end{array}$ & $\begin{array}{l}\text { wood chip } \\
\text { boiler }\end{array}$ & 50 & biomass & radiating floor & tiled stove \\
\hline 54 & Mairhof & IT & & wood boiler & not. & biomass & & \\
\hline 55 & Obergasserhof & IT & & heat pump & 32 & & radiating wall & $\begin{array}{l}\text { condensing } \\
\text { boiler }\end{array}$ \\
\hline 56 & Rebecco Farm & IT & gas & heat pump & 102. & & radiating ceiling & \\
\hline 57 & Villa Capodivacca & IT & & & 11 & biomass & $\begin{array}{l}\text { floor heating and } \\
\text { old stove }\end{array}$ & tiled stove \\
\hline 58 & House Moroder & SL & & pellet boiler & & biomass & radiators & \\
\hline 59 & Huberhof & SL & & $\begin{array}{l}\text { condensing } \\
\text { boiler }\end{array}$ & 48 & gas & radiators & \\
\hline 60 & Notarjeva vila & SL & $\begin{array}{l}\text { propane/ } \\
\text { butane }\end{array}$ & $\begin{array}{l}\text { wood chip } \\
\text { boiler }\end{array}$ & 45 & biomass & & \\
\hline 61 & Rožna ulica 15, Idrija & SL & & pellet boiler & 150 & biomass & radiating floor & \\
\hline 62 & $\begin{array}{l}\text { Hiša trentarskih } \\
\text { vodnikov }\end{array}$ & $\mathrm{SP}$ & & heat pump & 150 & geothermal & $\begin{array}{l}\text { radiant floor, fan } \\
\text { coils and } \mathrm{AHU}\end{array}$ & \\
\hline 63 & $\begin{array}{l}\text { Idrija mercury smelting } \\
\text { plant }\end{array}$ & $\mathrm{SP}$ & & heat pump & 325 & $\begin{array}{l}\text { air \& } \\
\text { electricity }\end{array}$ & radiating floor & \\
\hline 64 & $\begin{array}{l}\text { Mercado del Val, } \\
\text { Valladolid (Spain) }\end{array}$ & TK & $\begin{array}{l}\text { no } \\
\text { system }\end{array}$ & heat pump & & $\begin{array}{l}\text { air \& } \\
\text { electricity }\end{array}$ & $\begin{array}{l}\text { central air-to-air } \\
\text { heat pump }\end{array}$ & \\
\hline 65 & Correria 119 & TK & $\begin{array}{l}\text { no } \\
\text { system }\end{array}$ & heat pump & 100 & $\begin{array}{l}\text { air \& } \\
\text { electricity }\end{array}$ & $\begin{array}{l}\text { central air-to-air } \\
\text { heat pump }\end{array}$ & \\
\hline 66 & Ahmet Aga Mansion & UK & $\begin{array}{l}\text { open } \\
\text { fires }\end{array}$ & heat pump & 4.5 & geothermal & radiating floor & $\begin{array}{l}\text { wood burning } \\
\text { stove }\end{array}$ \\
\hline 67 & Necip Pasa Library & UK & & IR heating & 53 & electricity & radiating panel & \\
\hline 68 & Downie's Cottage & UK & & & & & & \\
\hline 69 & Hollyrood Park Lodge & USA & & condensing & & geothermal & air & \\
\hline
\end{tabular}




\subsubsection{Domestic hot water}

\subsubsection{Domestic hot water solutions}

In 18 cases there was no change of system for domestic hot water solutions, but in 40 cases it is connected with the heating system. It is interesting, that in 8 case studies the solution is combined with heat recovery (see Table 14).

Table 14: Overview of domestic hot water solutions

\begin{tabular}{|c|c|c|c|c|c|c|}
\hline No & Case study & 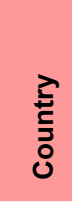 & Building type & System type & 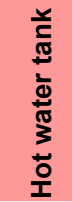 & 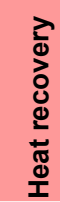 \\
\hline 1 & Klostergebäude Kaiserstrasse & AT & Residential (urban) & decentral boiler & yes & no \\
\hline 2 & Farm house Trins & AT & Residential (rural) & with heating system & yes & no \\
\hline 3 & Hof 6, Schwarzenberg & AT & Residential (rural) & RES & yes & no \\
\hline 4 & Kelchalm - Bochumer alpine hut & AT & Hotel/Restaurant & with heating system & yes & no \\
\hline 5 & Mariahilferstrasse 182 & AT & Residential (urban) & & & \\
\hline 6 & House Maurer, Wolfurt & AT & Residential (rural) & & & \\
\hline 7 & House Breuer, Tschagguns & AT & Residential (rural) & with heating system & yes & no \\
\hline 8 & Music school in Velden & AT & Educational/Research & & & \\
\hline 9 & Hof Neuhäusl & AT & Residential (rural) & with heating system & yes & no \\
\hline 10 & Community Hall Zwischenwasser & AT & Community Hall & decentral boiler & no & no \\
\hline 11 & Freihof Sulz & AT & Restaurant/Commerce & with heating system & yes & no \\
\hline 12 & Oeconomy building Josef Weiss & AT & Residential with Atelier & with heating system & yes & yes \\
\hline 13 & Giatla Haus & AT & Hotel/Restaurant & with heating system & yes & no \\
\hline 14 & Rhine Valley House Irgang & AT & Residential (rural) & RES & yes & no \\
\hline 15 & Baur Residence, Lustenau & AT & Residential (rural) & with heating system & yes & no \\
\hline 16 & Kasperhof & AT & Residential (rural) & with heating system & yes & no \\
\hline 17 & Maison Rubens & $\mathrm{BE}$ & Residential (urban) & decentral boiler & yes & yes \\
\hline 18 & Half-timberframed house in Alken & $\mathrm{BE}$ & Residential (rural) & with heating system & no & no \\
\hline 19 & Doragno Castle, Rovio & $\mathrm{CH}$ & Residential (rural) & with heating system & yes & no \\
\hline 20 & Solar silo & $\mathrm{CH}$ & Offices & & & \\
\hline 21 & Wohn- und Geschäftshaus & $\mathrm{CH}$ & Residential (urban) & with heating system & yes & yes \\
\hline 22 & Mehrfamilienhaus Magnusstrasse & $\mathrm{CH}$ & Residential (urban) & with heating system & yes & no \\
\hline 23 & St. Franziskus Church Ebmatingen & $\mathrm{CH}$ & Religious & with heating system & yes & no \\
\hline 24 & Kindergarten and apartments (PEB) & $\mathrm{CH}$ & Educational/Research & Solar & yes & no \\
\hline 25 & Single family home Luisenstrasse - & $\mathrm{CH}$ & Residential (urban) & with heating system & yes & yes \\
\hline 26 & Single Family House - Gstaad & $\mathrm{CH}$ & Residential (rural) & with heating system & yes & yes \\
\hline 27 & Glaserhaus in Affoltern & $\mathrm{CH}$ & Residential (rural) & with heating system & yes & no \\
\hline 28 & PalaCinema Locarno & $\mathrm{CH}$ & Platform for cinematic & with heating system & yes & yes \\
\hline 29 & Casa Rossa Chemnitz & $\mathrm{DE}$ & Residential (urban) & with heating system & yes & no \\
\hline 30 & Rathaus Bergrheinfeld & $\mathrm{DE}$ & Town Hall & & & \\
\hline 31 & Farmhouse Straub & $\mathrm{DE}$ & Residential (rural) & with heating system & yes & no \\
\hline 32 & Early work Sep Ruf & $\mathrm{DE}$ & Residential (rural) & Local heat & yes & no \\
\hline 33 & Ackerbürgerhäuschen & $\mathrm{DE}$ & Residential (rural) & with heating system & yes & no \\
\hline
\end{tabular}




\begin{tabular}{|c|c|c|c|c|c|c|}
\hline 34 & Ritterhof & $\mathrm{DE}$ & Residential (rural) & with heating system & yes & no \\
\hline 35 & Rathaus Burgkunstadt & $\mathrm{DE}$ & Town Hall & decentral boiler & no & no \\
\hline 36 & Osramhuset & DK & Culture \& Community & & & \\
\hline 37 & Klitgaarden & DK & Residential (rural) & & & \\
\hline 38 & RYESGADE 30 & DK & Residential (urban) & & & \\
\hline 39 & Timber-framed house in Alsace & FR & Residential (rural) & with heating system & yes & no \\
\hline 40 & Elementary School in Mulhouse & FR & Education/Research & & & \\
\hline 41 & Timber-framed barn in the north of & FR & Residential (rural) & decentral boiler & yes & no \\
\hline 42 & Rainhof & IT & Residence (rural) & with heating system & yes & no \\
\hline 43 & Villa Castelli & IT & Residential (rural) & with heating system & yes & no \\
\hline 44 & Ansitz Kofler & IT & Residential (urban) & with heating system & yes & no \\
\hline 45 & Basilica di Santa Maria di & IT & Religious & & & \\
\hline 46 & House Pernter & IT & Residential (rural) & with heating system & yes & no \\
\hline 47 & Kohlerhaus & IT & Residential (urban) & with heating system & no & no \\
\hline 48 & Ruckenzaunerhof & IT & Residential (rural) & & & \\
\hline 49 & Aussergrubhof & IT & Residential (rural) & with heating system & yes & no \\
\hline 50 & Oberbergerhof & IT & Residential (rural) & with heating system & yes & no \\
\hline 51 & Platzbonhof & IT & Residential (rural) & with heating system & yes & no \\
\hline 52 & Mairhof & IT & Residential (rural) & RES & yes & no \\
\hline 53 & Obergasserhof & IT & Residential (rural) & with heating system & no & no \\
\hline 54 & Rebecco Farm & IT & B\&B - Farm & & & \\
\hline 55 & Villa Capodivacca & IT & Residential (urban) & with heating system & yes & no \\
\hline 56 & House Moroder & IT & Residential (urban) & with heating system & & \\
\hline 57 & Huberhof & IT & Residential (rural) & with heating system & yes & no \\
\hline 58 & Notarjeva vila & $S L$ & Residential (urban) & air - water & 0 & 0 \\
\hline 59 & Rožna ulica 15 , Idrija & SL & Residential (urban) & with heating system & yes & no \\
\hline 60 & Hiša trentarskih vodnikov & SL & Multipurpose & with heating system & yes & yes \\
\hline 61 & Idrija mercury smelting plant & SL & Educational/Research & with heating system & yes & no \\
\hline 62 & Mercado del Val, Valladolid & SP & Wholesale and Retail & with heating system & yes & yes \\
\hline 63 & Correria 119 & $\mathrm{SP}$ & Residential (urban) & with heating system & & \\
\hline 64 & Ahmet Aga Mansion & TK & Offices & & & \\
\hline 65 & Nwcip Pasa Library & TK & Library & & & \\
\hline 66 & Downie's Cottage & UK & Residential (rural) & Immersion heater & yes & no \\
\hline 67 & Hollyrood Park Lodge & UK & Wholesale and Retail & & & \\
\hline 68 & Annat Road & UK & Residential (urban) & & & \\
\hline 69 & Aspinall Courthouse & US & Offices & with heating system & yes & no \\
\hline
\end{tabular}

\subsubsection{Ventilation}

\subsubsection{Overview of ventilation solutions}

Table 15: Overview - ventilation solutions

\begin{tabular}{|c|c|c|c|c|c|}
\hline No & Case study & 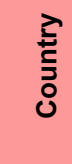 & Building type & Intervention & 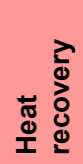 \\
\hline 1 & $\begin{array}{l}\text { Klostergebäude } \\
\text { Kaiserstrasse }\end{array}$ & AT & Residential (urban) & $\begin{array}{l}\text { Semi-centralised MVHR, distribution with } \\
\text { exist shafts }\end{array}$ & yes \\
\hline
\end{tabular}




\begin{tabular}{|c|c|c|c|c|c|}
\hline 2 & Farm house Trins & AT & Residential (rural) & centralised MVHR with cascade system & yes \\
\hline 3 & Hof 6, Schwarzenberg & AT & Residential (rural) & & \\
\hline 4 & Kelchalm & AT & Hotel/Restaurant & decentralised MVHR for laundry rooms & yes \\
\hline 5 & Mariahilferstrasse 182 & AT & Residential (urban) & centralised MVHR with bypass & yes \\
\hline 6 & House Maurer, Wolfurt & AT & Residential (rural) & & \\
\hline 7 & House Breuer, Tschagguns & AT & Residential (rural) & centralised MVHR & yes \\
\hline 8 & Music school in Velden & AT & Educational/Research & $\begin{array}{l}2 \text { centralised (from attic and using } \\
\text { existing chimneys) \& } 1 \text { decentralised } \\
\text { systems }\end{array}$ & yes \\
\hline 9 & Hof Neuhäusl & AT & Residential (rural) & centralised MVHR & yes \\
\hline 10 & $\begin{array}{l}\text { Community Hall } \\
\text { Zwischenwasser }\end{array}$ & AT & Community Hall & centralised MVHR with cascade system & yes \\
\hline 11 & Freihof Sulz & AT & Restaurant/Commerce & centralised MVHR & yes \\
\hline 12 & $\begin{array}{l}\text { Oeconomy building Josef } \\
\text { Weiss }\end{array}$ & AT & $\begin{array}{l}\text { Residential with } \\
\text { Atelier }\end{array}$ & $\begin{array}{l}\text { centralised MVHR for kitchen, restaurant } \\
\& \text { dining room }\end{array}$ & yes \\
\hline 13 & Giatla Haus & AT & Hotel/Restaurant & centralised MVHR with humidity control & yes \\
\hline 14 & Rhine Valley House Irgang & AT & Residential (rural) & & \\
\hline 15 & Baur Residence, Lustenau & AT & Residential (rural) & & \\
\hline 16 & Kasperhof & AT & Residential (rural) & & \\
\hline 17 & Maison Rubens & $\mathrm{BE}$ & Residential (urban) & centralised MVHR using chimneys & yes \\
\hline 18 & $\begin{array}{l}\text { Half-timberframed house in } \\
\text { Alken }\end{array}$ & $\mathrm{BE}$ & Residential (rural) & centralised MVHR & yes \\
\hline 19 & Doragno Castle, Rovio & $\mathrm{CH}$ & Residential (rural) & centralised MVHR & yes \\
\hline 20 & Solar silo & $\mathrm{CH}$ & Offices & natural ventilation, manual via windows & no \\
\hline 21 & $\begin{array}{l}\text { Wohn- und Geschäftshaus } \\
\text { Feldbergstrasse }\end{array}$ & $\mathrm{CH}$ & Residential (urban) & centralised MVHR & yes \\
\hline 22 & $\begin{array}{l}\text { Mehrfamilienhaus } \\
\text { Magnusstrasse }\end{array}$ & $\mathrm{CH}$ & Residential (urban) & centralised MVHR & yes \\
\hline 23 & $\begin{array}{l}\text { St. Franziskus Church } \\
\text { Ebmatingen }\end{array}$ & $\mathrm{CH}$ & Religious & & \\
\hline 24 & $\begin{array}{l}\text { Kindergarten and apartments } \\
\text { (PEB) Chur }\end{array}$ & $\mathrm{CH}$ & Educational/Research & decentralised MVHR, night cooling & yes \\
\hline 25 & $\begin{array}{l}\text { Single family home } \\
\text { Luisenstrasse - Bern }\end{array}$ & $\mathrm{CH}$ & Residential (urban) & $\begin{array}{l}\text { centralised MVHR with screed in built-in } \\
\text { cupboards }\end{array}$ & yes \\
\hline 26 & Single Family House - Gstaad & $\mathrm{CH}$ & Residential (rural) & & \\
\hline 27 & Glaserhaus in Affoltern & $\mathrm{CH}$ & Residential (rural) & natural ventilation via windows) & no \\
\hline 28 & PalaCinema Locarno & $\mathrm{CH}$ & Platform for cinematic & centralised MVHR, differentiated use & yes \\
\hline 29 & Casa Rossa Chemnitz & DE & Residential (urban) & Exhaust ventilation & no \\
\hline 30 & Rathaus Bergrheinfeld & DE & Town Hall & Centralised MVHR & yes \\
\hline 31 & Farmhouse Straub & DE & Residential (rural) & & \\
\hline 32 & Early work Sep Ruf & DE & Residential (rural) & & \\
\hline 33 & Ackerbürgerhäuschen & DE & Residential (rural) & centralised MVHR & yes \\
\hline 34 & Ritterhof & $\mathrm{DE}$ & Residential (rural) & centralised MVHR for part of the building & yes \\
\hline 35 & Rathaus Burgkunstadt & DE & Town Hall & $\begin{array}{l}\text { centralised MVHR from attic for offices } \\
\text { (in top floor) }\end{array}$ & yes \\
\hline 36 & Osramhuset & DK & $\begin{array}{l}\text { Culture \& Community } \\
\text { Centre }\end{array}$ & $\begin{array}{l}\text { centralised MVHR combined with natural } \\
\text { ventilation }\end{array}$ & \\
\hline 37 & Klitgaarden & DK & Residential (rural) & $\begin{array}{l}\text { centralised mechanical ventilation with } \\
\text { heat recovery }\end{array}$ & yes \\
\hline 38 & RYESGADE 30 & DK & Residential (urban) & 2 central one decentral MVHR & yes \\
\hline 39 & $\begin{array}{l}\text { Timber-framed house in } \\
\text { Alsace }\end{array}$ & $\mathrm{FR}$ & Residential (rural) & centralised MVHR, hidden & yes \\
\hline
\end{tabular}




\begin{tabular}{|c|c|c|c|c|c|}
\hline 40 & $\begin{array}{l}\text { Elementary School in } \\
\text { Mulhouse }\end{array}$ & FR & Education/Research & centralised MVHR with bypass & yes \\
\hline 41 & $\begin{array}{l}\text { Timber-framed barn in the } \\
\text { north of France }\end{array}$ & FR & Residential (rural) & exhaust ventilation & no \\
\hline 42 & Rainhof & IT & Residence (rural) & & \\
\hline 43 & Villa Castelli & IT & Residential (rural) & decentralised MVHR & yes \\
\hline 44 & Ansitz Kofler & IT & Residential (urban) & centralised MVHR with summer bypass & yes \\
\hline 45 & Basilica di Santa Maria di & IT & Religious & & \\
\hline 46 & House Pernter & IT & Residential (rural) & decentralised MVHR & yes \\
\hline 47 & Kohlerhaus & IT & Residential (urban) & room-by-room MVHR & yes \\
\hline 48 & Ruckenzaunerhof & IT & Residential (rural) & & \\
\hline 49 & Aussergrubhof & IT & Residential (rural) & & \\
\hline 50 & Oberbergerhof & IT & Residential (rural) & & \\
\hline 51 & Platzbonhof & IT & Residential (rural) & & \\
\hline 52 & Mairhof & IT & Residential (rural) & centralised MVHR & yes \\
\hline 53 & Obergasserhof & IT & Residential (rural) & & \\
\hline 54 & Rebecco Farm & IT & B\&B - Farm & & \\
\hline 55 & Villa Capodivacca & IT & Residential (urban) & centralised MVHR & yes \\
\hline 56 & House Moroder & IT & Residential (urban) & decentralised MVHR & yes \\
\hline 57 & Huberhof & IT & Residential (rural) & & \\
\hline 58 & Notarjeva vila & SL & Residential (urban) & & \\
\hline 59 & Rožna ulica 15, Idrija & SL & Residential (urban) & & \\
\hline 60 & Hiša trentarskih vodnikov & SL & Multipurpose & & \\
\hline 61 & Idrija mercury smelting plant & SL & Educational/Research & & \\
\hline 62 & Mercado del Val, Valladolid & SP & Wholesale and Retail & controlled natural ventilation \& AHU & yes \\
\hline 63 & Correria 119 & SP & Residential (urban) & & \\
\hline 64 & Ahmet Aga Mansion & TK & Offices & & \\
\hline 65 & Nwcip Pasa Library & TK & Library & centralised MVHR & \\
\hline 66 & Downie's Cottage & UK & Residential (rural) & & \\
\hline 67 & Hollyrood Park Lodge & UK & Wholesale and Retail & $\begin{array}{l}\text { natural Ventilation via historic flues and } \\
\text { hearth }\end{array}$ & no \\
\hline 68 & Annat Road & UK & Residential (urban) & $\begin{array}{l}\text { natural Ventilation via historic flues and } \\
\text { hearth }\end{array}$ & no \\
\hline 69 & Aspinall Courthouse & USA & Offices & & \\
\hline
\end{tabular}

In 41 of the 69 renovated buildings explicit information on the ventilation is provided: Actually, 4 of them have natural ventilation and authors use the tab to describe the deliberate decision, in two of the cases also pointing out how openings were reinstalled to allow for good natural ventilation. 2 buildings got an exhaust ventilation system and the remaining 35 buildings (i.e. 50\%) are equipped with a mechanical ventilation system with heat recovery (MVHR). Thereof 1 is a room-by-room system, 5 are decentralized systems, 26 central systems and in 2 cases both central and decentralized systems were installed (in different parts of the building, also to study performance, as well as pros and cons). There is also one case where a central mechanical ventilation with heat recovery is coupled with natural ventilation.

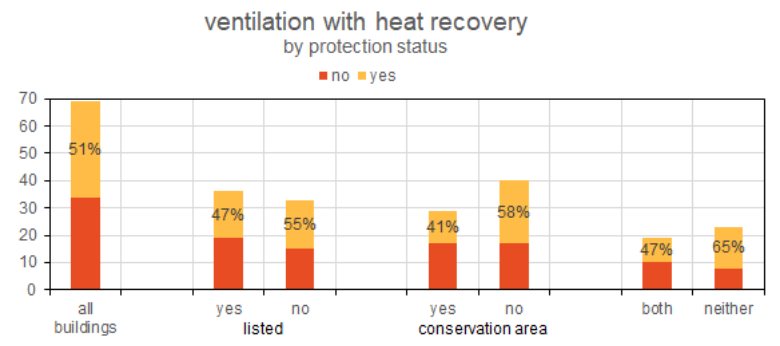


Figure 53: Ventilation with heat recovery by main use in absolute numbers (left) and share (mid) as well as by both considering main and secondary use (right)

35 buildings with MVHR correspond to slightly more than half of all the documented good practice buildings. The fact that looking specifically at listed buildings, $47 \%$ have been equipped with a ventilation with heat recovery, induces, that it might be slightly but not considerably more difficult to integrate a ventilation system in a listed building. The analysis looking at whether buildings are situated in conservation areas or are both - listed and in a conservation area - shows the same trend.
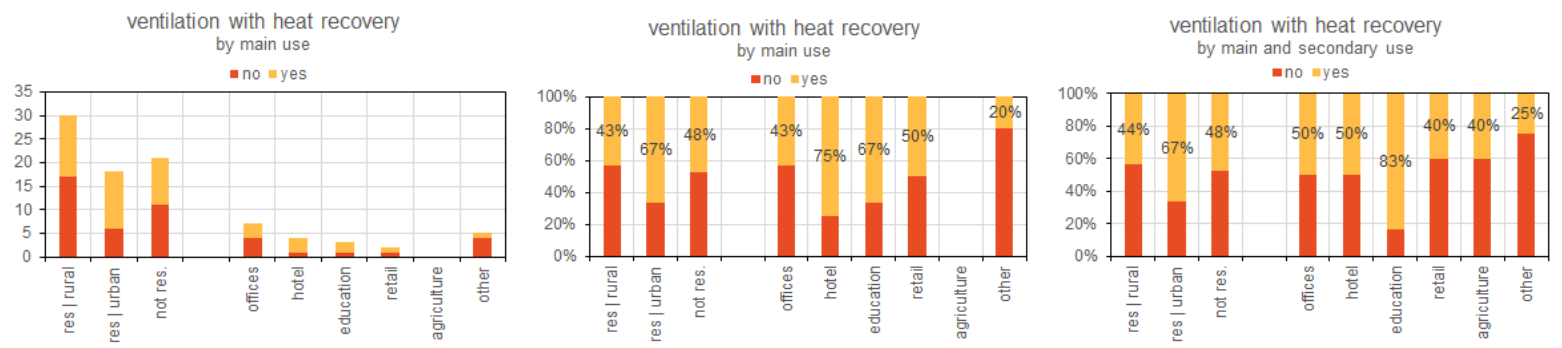

Figure 54: Ventilation with heat recovery by main use in absolute numbers (left) and share (mid) as well as by both considering main and secondary use (right)

Ventilation systems have more often be considered in retrofits of urban than rural residential buildings: the number of documented ventilation systems is with 12 each equivalent, but since overall, less urban residential buildings have been documented, the share is with of 2 out of 3 retrofits with ventilation system highest there. This value is only topped, when looking explicitly at buildings with any kind of educational use: 2 out 3 with it as main use have a ventilation system, including those with a secondary educational use the numbers rise to 4 out of 5 - this shows a clear sensibility for indoor air quality in these cases.
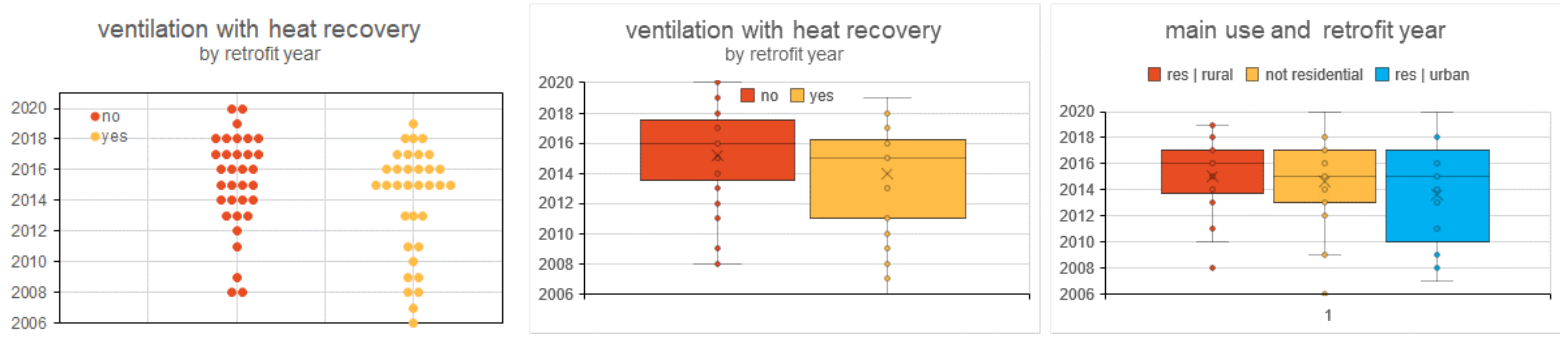

Figure 55: Year of retrofit - by ventilation with heat recovery (left) and main use (right)

The hypothesis, that recent retrofits might more often include MVHR since experience on how to implement it is rising is not supported by the collected data: the whisker plot of retrofits with and without MVHR actually shows that those with MVHR are on average "older". This could, however, also be due to the fact that there is dominance of rural residential retrofits in the documented recent cases, which as has been shown above are less likely to be equipped with MVHR.
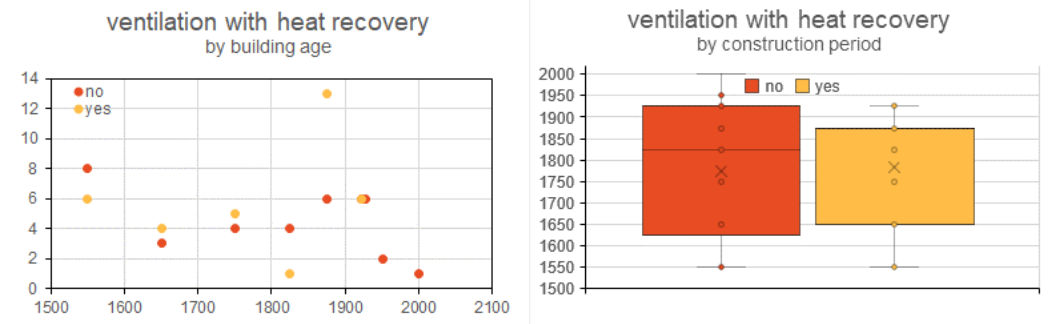

Figure 56: Differences by construction period

There is also no evidence in the data, that the implementation of a MVHR is more difficult in older buildings. The whisker plot (see Figure 56 on the right) shows very similar values for the "average age" of buildings with and 
without MVHR, the range just being wider for those without. This can also be observed in the left plot: both for the newest and the oldest buildings slightly less buildings were retrofitted with MVHR. A particularly high share of retrofits with MVHR can be observed for buildings dating $1850-1900$.
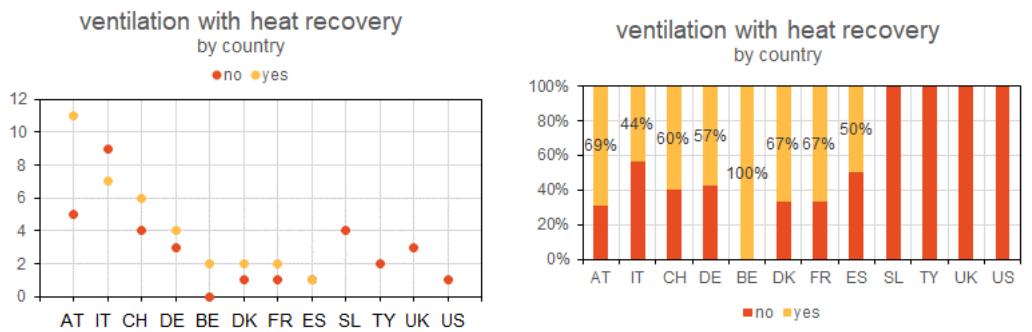

Figure 57: Differences by country

Investigating whether there can be observed differences from country to country, shows on the one hand side, that most best practice cases with MVHR have been reported for Austria, followed by Italy and Switzerland. It is worth noticing however, that all best practice from Belgium have a MVHR, followed by Austria, Denmark and France with two thirds. On the other hand, none of the cases documented for Slovenia, Turkey, the United Kingdom and the states was equipped with MVHR.

\subsubsection{Technical overview}

As the data and documentation of the ventilation systems are partly incomplete, only a few facts of the fully documented examples are given below.

In most cases, 26 (resp. 28) out of 35, the ventilation system is centralized. In one case, "Ryesgade" [38], different systems have been implemented in order to study pros and cons and learn for future implementations: a traditional central system $(A)$, a central system with demand control (B) and a decentral system (C). At "Musikschule Velden" [8] different systems were installed for different areas: one central system for the class rooms $(A)$, another central unit for event hall and assembly hall $(B)$ and finally decentralised units for the sanitary facilities (C). Additionally, to those two, decentral systems have been implemented at three Italian cases ("Villa Castelli" [43], "Haus Pernter" [46] and "Haus Moroder" [56]), the Austrian "Kelchalm - Bochumer Hütte" [4] and the Swiss "Kindergarten and apartments - Chur" [24]. For the latter this allowed and optimsed operation adapted to the occupancy - and night cooling in the kindergarten - and also for reduced ducting with MVHR units of the apartments in the bathrooms. The only case with documented room-by-room system is the "Kohlerhaus" [47].

In terms of control of the ventilation system, 4 cases explicitly report control based on $\mathrm{CO}_{2}$ : "Villa Castelli" [43) and "Kelchalm" [4] as well as "Giatla Haus" [13] and "Rathaus Bergrheinfeld" [30] - the latter two mentioning also humidity as additional parameter for control.

In the case of "Mercado del Val" [62] a natural ventilation system using windows and skylights is controlled by one BEMS together with the air handling units - which only switch on, when natural ventilation is not enough. In two of the three UK cases natural ventilation is enhanced by re-installing traditional elements and allowing for ventilation via the hearth and chimney, or an adjustable quarter light ("Hollyrood Park Lodge" [67] and "Annat Road" [68]). And at "Osramhuset" [36] the original exhaust system where air was removed from toilets and kitchens was replaced by a mechanical ventilation with heat recovery, supplemented by natural ventilation via the roof windows which are operated by electric motors based on the indoor climate.

The information on the flow regime is not complete, but 9 cases report to have implemented a cascade system in order to avoid ducting: "Bauernhof Trins" [2], "Hof Neuhäusl" [9], "Gemeindehaus Zwischenwasser" [10], "Freihof Sulz"[11] and "Giatla Haus" [13] in Austria, "Ryesgade" [38] in Denmark, the "timber-framed house in Alsace" [39] and the "Elementary school Mulhouse" [40] in France, and finally "Ansitz Mairhof" [52] in Italy.

\subsubsection{Heritage compatibility}

Ventilation is an often discussed not easy issue, especially in listed buildings. It is undisputed that ventilation is important and should be considered in every refurbishment project - for comfort and health reasons, energy saving and climate change mitigation but especially because it can support the long term preservation of the building fabric by decreasing the interstitial condensation risk as well as mould growth: by keeping relative humidity of the indoor air low and possibly also guaranteeing a slight underpressure inside the building, which 
avoids humid air penetrating into walls. That these reasons lead to the decision for ventilation systems - or at least a very deliberate consideration of the guaranteed natural ventilation has been pointed out at several best practice cases.

With regard to the protection of historical monuments, the ventilation must be as invisible as possible. Active overflow solutions and cascade ventilation considerably reduce the amount of pipework. For these old buildings you also have to be a little inventive in how to hide the air supply and exhaust air pipes. Old chimney flues and existing shafts can be used. For the "Farm house Trins" the air vents were hidden in the suspended wooden ceiling and integrated as unobtrusively as possible (figure 56). In the case of the timber-framed house in Alsace, the pipes were concealed and plastered between the joists. The owner did not want to see the exhaust and air intake vents on the roof, so he hid them behind shutters and near an exterior staircase. Attics can be used specifically for the ventilation units, as the example in figure 58 shows.

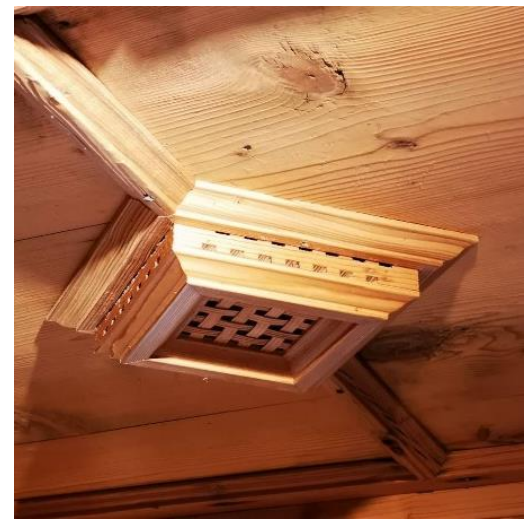

Figure 58: farm house Trins, ventilation ducts

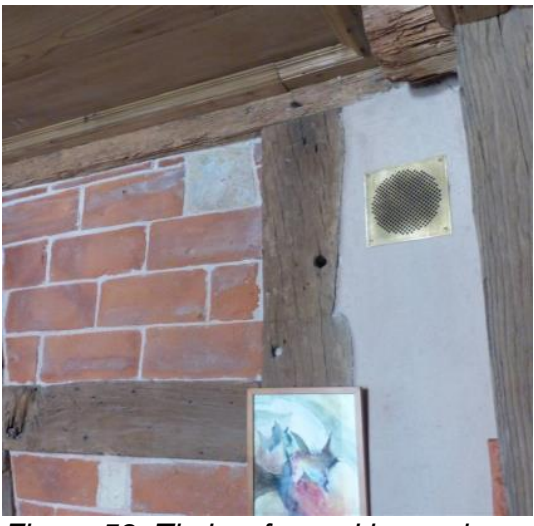

Figure 59: Timber-framed house in Alsace, supply air invisible between joists

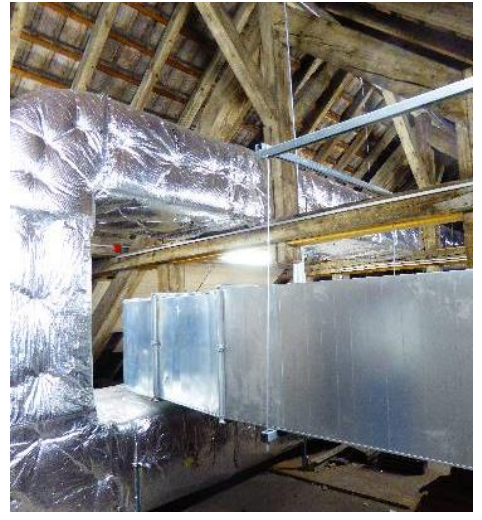

Figure 60: Elementary School "Cour de Larraine", dual flow units in the attic

\subsection{Renewable energy systems}

\subsubsection{Overview of renewable energy solutions}

In the 69 cases analysed there are 45 cases, which include renewable energy solutions and 24 which do not. In the cases where renewable energy has been implemented, most often several different types of solutions are combined, e.g. in Villa Castelli where photovoltaics, wind and geothermal energy were installed to complement the renovation of the building envelope and the building systems. The following table gives an overview of the solutions.

Table 16: Types of renewable energy solutions in case studies

\begin{tabular}{|c|c|c|c|c|c|c|c|c|}
\hline No & Case study & 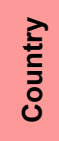 & Building type & 흘 & ฉ & $\stackrel{\text { 을 }}{3}$ & ò & $\begin{array}{l}\text { g) } \\
\frac{0}{0} \\
\frac{8}{0} \\
\frac{0}{m}\end{array}$ \\
\hline 1 & Klostergebäude Kaiserstrasse & AT & Residential (urban) & & & & & \\
\hline 2 & Farm house Trins & AT & Residential (rural) & & & & 1 & 1 \\
\hline 3 & Hof 6, Schwarzenberg & AT & Residential (rural) & 1 & & & & 1 \\
\hline 4 & Kelchalm & AT & Hotel/Restaurant & 1 & & & & 1 \\
\hline 5 & Mariahilferstrasse 182 & AT & Residential (urban) & 1 & & & & \\
\hline 6 & House Maurer, Wolfurt & AT & Residential (rural) & 1 & & & & \\
\hline 7 & House Breuer, Tschagguns & AT & Residential (rural) & 1 & 1 & & & 1 \\
\hline 8 & Music school in Velden & AT & Educational/Research & & 1 & & & \\
\hline 9 & Hof NeuhäusI & AT & Residential (rural) & & & & & \\
\hline 10 & Community Hall Zwischenwasser & AT & Community Hall & & & & & \\
\hline
\end{tabular}




\begin{tabular}{|c|c|c|c|c|c|c|c|c|}
\hline 11 & Freihof Sulz & AT & Restaurant/Commerce & 1 & & & & \\
\hline 12 & Oeconomy building Josef Weiss & AT & Residential with Atelier & & & & & \\
\hline 13 & Giatla Haus & AT & Hotel/Restaurant & & & & & 1 \\
\hline 14 & Rhine Valley House Irgang & AT & Residential (rural) & 1 & & & & 1 \\
\hline 15 & Baur Residence, Lustenau & AT & Residential (rural) & 1 & & & & 1 \\
\hline 16 & Kasperhof & AT & Residential (rural) & & & & & \\
\hline 17 & Maison Rubens & $\mathrm{BE}$ & Residential (urban) & 1 & & & & 1 \\
\hline 18 & Half-timberframed house in Alken & $\mathrm{BE}$ & Residential (rural) & & & & & \\
\hline 19 & Doragno Castle, Rovio & $\mathrm{CH}$ & Residential (rural) & 1 & 1 & & & \\
\hline 20 & Solar silo & $\mathrm{CH}$ & Offices & & 1 & & & \\
\hline 21 & $\begin{array}{l}\text { Wohn- und Geschäftshaus } \\
\text { Feldbergstrasse }\end{array}$ & $\mathrm{CH}$ & Residential (urban) & 1 & 1 & & & \\
\hline 22 & Mehrfamilienhaus Magnusstrasse & $\mathrm{CH}$ & Residential (urban) & 1 & & & & 1 \\
\hline 23 & St. Franziskus Church Ebmatingen & $\mathrm{CH}$ & Religious & 1 & 1 & & 1 & \\
\hline 24 & $\begin{array}{l}\text { Kindergarten and apartments (PEB) } \\
\text { Chur }\end{array}$ & $\mathrm{CH}$ & Educational/Research & 1 & 1 & & & 1 \\
\hline 25 & $\begin{array}{l}\text { Single family home Luisenstrasse - } \\
\text { Bern }\end{array}$ & $\mathrm{CH}$ & Residential (urban) & 1 & 1 & & 1 & 1 \\
\hline 26 & Single Family House - Gstaad & $\mathrm{CH}$ & Residential (rural) & & 1 & & & \\
\hline 27 & Glaserhaus in Affoltern & $\mathrm{CH}$ & Residential (rural) & & 1 & & 1 & \\
\hline 28 & PalaCinema Locarno & $\mathrm{CH}$ & $\begin{array}{l}\text { Platform for cinematic } \\
\text { arts }\end{array}$ & & 1 & & 1 & \\
\hline 29 & Casa Rossa Chemnitz & DE & Residential (urban) & 1 & & & & \\
\hline 30 & Rathaus Bergrheinfeld & DE & Town Hall & & & & & \\
\hline 31 & Farmhouse Straub & DE & Residential (rural) & 1 & 1 & & & \\
\hline 32 & Early work Sep Ruf & DE & Residential (rural) & & & & & 1 \\
\hline 33 & Ackerbürgerhäuschen & DE & Residential (rural) & & & & & \\
\hline 34 & Ritterhof & DE & Residential (rural) & 1 & 1 & & & 1 \\
\hline 35 & Rathaus Burgkunstadt & DE & Town Hall & & & & & \\
\hline 36 & Osramhuset & DK & $\begin{array}{l}\text { Culture \& Community } \\
\text { Centre }\end{array}$ & 1 & & & & \\
\hline 37 & Klitgaarden & DK & Residential (rural) & & & & & \\
\hline 38 & RYESGADE 30 & DK & Residential (urban) & & 1 & & & \\
\hline 39 & Timber-framed house in Alsace & FR & Residential (rural) & & & & & 1 \\
\hline 40 & Elementary School in Mulhouse & FR & Education/Research & & & & & \\
\hline 41 & $\begin{array}{l}\text { Timber-framed barn in the north of } \\
\text { France }\end{array}$ & $\mathrm{FR}$ & Residential (rural) & & & & & 1 \\
\hline 42 & Rainhof & IT & Residence (rural) & & & & & 1 \\
\hline 43 & Villa Castelli & IT & Residential (rural) & & 1 & 1 & 1 & \\
\hline 44 & Ansitz Kofler & IT & Residential (urban) & 1 & & & 1 & 1 \\
\hline 45 & $\begin{array}{l}\text { Basilica di Santa Maria di } \\
\text { Collemaggio }\end{array}$ & $\mathrm{IT}$ & Religious & & & & 1 & \\
\hline 46 & House Pernter & IT & Residential (rural) & 1 & & & & 1 \\
\hline 47 & Kohlerhaus & IT & Residential (urban) & & & & & \\
\hline 48 & Ruckenzaunerhof & IT & Residential (rural) & & & & & \\
\hline 49 & Aussergrubhof & IT & Residential (rural) & & & & & 1 \\
\hline 50 & Oberbergerhof & IT & Residential (rural) & & 1 & & & 1 \\
\hline 51 & Platzbonhof & IT & Residential (rural) & & & & & \\
\hline 52 & Mairhof & IT & Residential (rural) & 1 & 1 & & & 1 \\
\hline 53 & Obergasserhof & IT & Residential (rural) & & & & & \\
\hline 54 & Rebecco Farm & IT & $\mathrm{B} \& \mathrm{~B}$ - Farm & & & & & 1 \\
\hline 55 & Villa Capodivacca & IT & Residential (urban) & & & & & \\
\hline 56 & House Moroder & IT & Residential (urban) & & & & & \\
\hline 57 & Huberhof & IT & Residential (rural) & & 1 & & & \\
\hline 58 & Notarjeva vila & SL & Residential (urban) & & & & & 1 \\
\hline 59 & Rožna ulica 15 , Idrija & SL & Residential (urban) & & & & & \\
\hline
\end{tabular}




\begin{tabular}{|l|l|l|l|l|l|l|}
\hline 60 & Hiša trentarskih vodnikov & SL & Multipurpose & & \\
\hline 61 & Idrija mercury smelting plant & SL & Educational/Research & & \\
\hline 62 & Mercado del Val, Valladolid & SP & Wholesale and Retail & & \\
\hline 63 & Correria 119 & SP & Residential (urban) & & \\
\hline 64 & Ahmet Aga Mansion & TK & Offices & & \\
\hline 65 & Nwcip Pasa Library & TK & Library & & \\
\hline 66 & Downie's Cottage & UK & Residential (rural) & & \\
\hline 67 & Hollyrood Park Lodge & UK & Wholesale and Retail & & \\
\hline 68 & Annat Road & USA & Offices & & \\
\hline 69 & Aspinall Courthouse & & & & \\
\hline & Total & & & & \\
\hline
\end{tabular}

From the table it is clear that 22 projects rely on only one renewable energy technology, 15 projects combine two different technologies, 7 projects combine three different technologies and one project (Single family house Luissenstrasse - Bern) combines four renewable energy technologies. There is no specific combinations of technologies or single technologies that are favoured more often than others. Wind technology is only used in one case, i.e. Villa Castelli where micro wind turbines where installed on the roof. The micro wind turbines contribute to the electricity production in combination with photovoltaics; unfortunately, there is no data on production or specifics on turbines etc. Wind energy is not discussed further in this report.

Figure 59 shows the total number of buildings and the number of buildings in each category that utilizes the different types of renewable energy sources. If we compare the setting of the buildings (15 urban / 30 rural) and the use of renewable energy sources, it is clear that in particular photovoltaics and biomass is more often favoured in a rural setting.

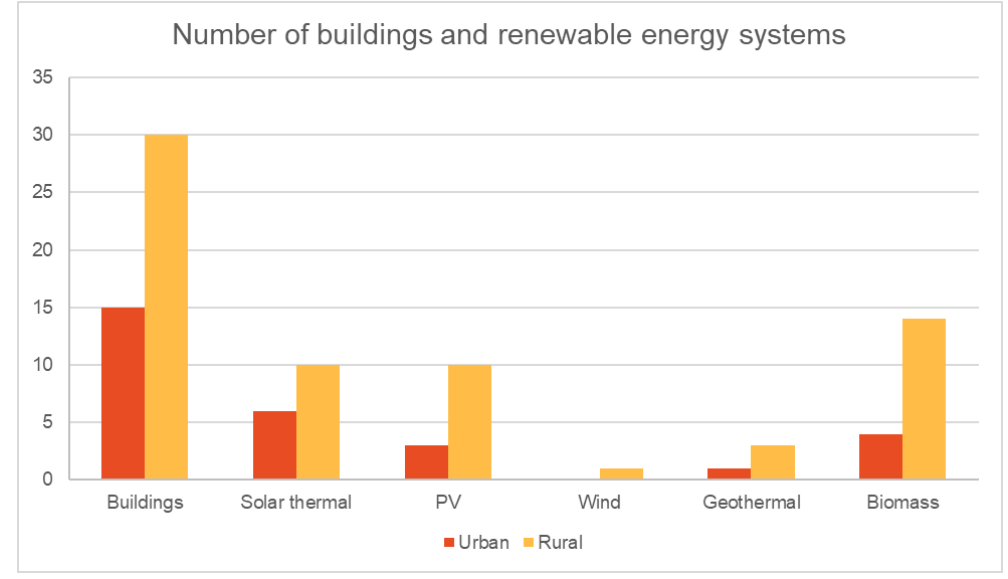

Figure 61: Urban and rural buildings and distribution of renewable energy systems.

\subsubsection{Solar thermal}

A solar heating system consists of a solar panel (typically located on a roof of the building). The panel contains a number of pipes with water and when the sun hits the panel, it heats the water. The hot water is fed directly into the buildings central heating system and can then be used for either domestic hot water, heating or both. Usually solar heat cannot act as the sole source of heat for a building, and therefore solar heating is typically used as a supplement to heating in buildings with electric heating or oil furnaces and allows to completely switch off these energy sources during the summer months.

Among the analysed cases there are 22 that have implemented solar thermal, below some examples are described.

In Osramhuset $4.8 \mathrm{~m}^{2}$ solar collectors were added to the roof. This was possible since the roof of the buildings was not part of the heritage value. The solar thermal installation only contributes to the production of domestic hot water for the building and supplies approx. $10 \mathrm{kWh} / \mathrm{m}^{2}$ floor area per year. 
In Ansitz Kopler no solar thermal was installed yet due to conservation restrictions. However, the storage for DHW has a two-coil type heat exchanger - to allow for the integration of a solar circuit in the future. A system will be installed when aesthetics of solar panels have been further developed to fit in conservation areas.

In Doragno Castle solar thermal collectors were integrated in the south-east roof pitch for the production of domestic hot water and heating. The selected roofing materials allowed for the integration of solar systems making a uniform surface, considering the aesthetic characteristics of the solar thermal panels and their geometry and position in the roof, i.e. the solar thermal modules are coplanar to the roof minimizing their visibility from the surrounding environment.

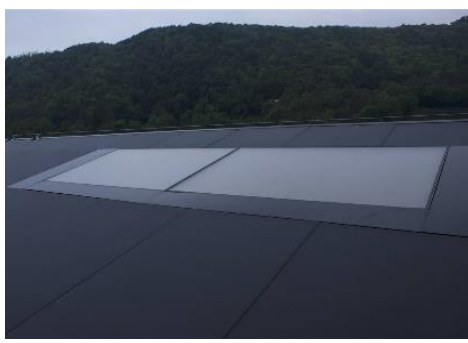

Figure 62: Integration of solar thermal panels in the roof of Doragno Castle.

Maison Rubens installed $5.4 \mathrm{~m}^{2}$ solar collectors. Unfortunately, no further information is available for this case, i.e. regarding placement, expected production etc.

In Ansitz Mairhof a solar thermal collector was installed on the roof of the new apartment (i.e. an extension of the existing building). Since the system was not installed on the listed residential building, but on the new extension on the roof, it was approved. The system has a total of $20 \mathrm{~m}^{2}$ collector area and is expected to produce 8,000 kWh per year.

In Mariahilferstrasse a $30 \mathrm{~m}^{2}$ solar thermal plant was installed on the roof. The plant supports the production of domestic hot water for the building. The new solar thermal system did not interfere with the conservation compatibility.

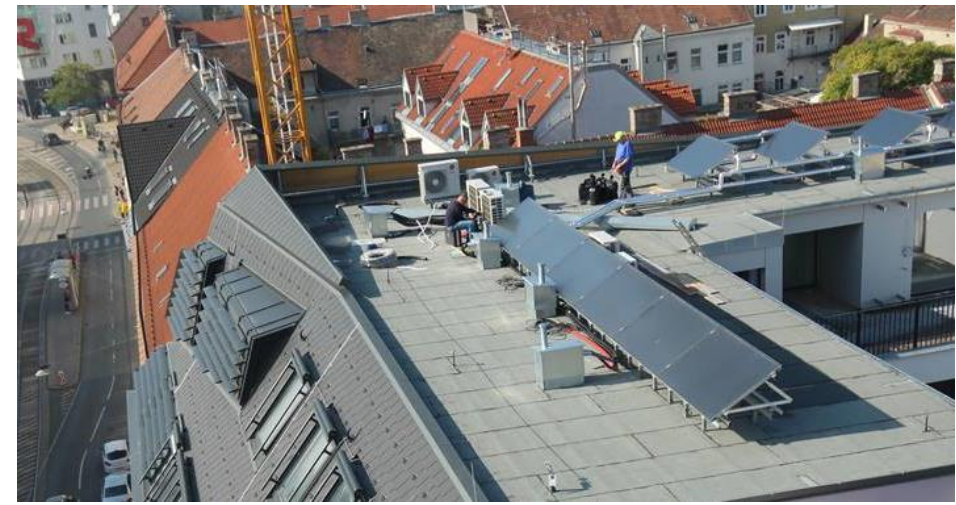

Figure 63: Mariahilferstrasse. Solar thermal installation on the roof.

In Casa Rossa Chemnitz the colour of the solar collectors was adapted to the roof skin. A rooftop system was chosen to enable simple reversibility of the technical component as soon as there are fully compatible components in terms of design, which is often a requirement for monument protection. Together with the roof windows, they represent a negligible disturbance of the roof landscape. 


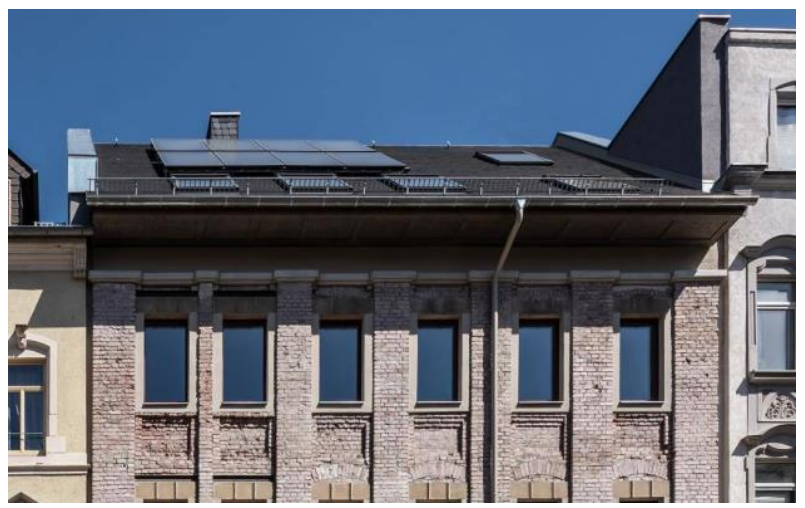

Figure 64: Casa Rossa Chemnitz. Coloured Solar collector colour adapted to roof skin. (Source: Bodensteiner Fest Architekten BDA).

Adding solar thermal to historic buildings is a way to increase energy efficiency by utilizing renewable energy (replacing the use of fossil fuels). The main part of the plant is a solar panel and optimally this needs to be placed where there is solar irradiance. For historic buildings, this can sometimes be achieved on roofs that are e.g. not visible from the ground or on parts of the building that is not protected. In the analysed cases, all plants were placed on roofs. In Osramhuset, there were no restrictions on the roof and in Doragno Castle, the panels were fitted perfectly in the roof construction, making it less visible from the ground. In Ansitz Mairhof, the plant was placed on a new part of the building that was not listed.

\subsubsection{Photovoltaics}

Photovoltaic panels, or PV-panels, convert solar irradiance into electricity. The plant typically consists of a series of panels that are placed on e.g. the roof of the building, and these panels are connected to electronic equipment (inverter, power conditioning unit, grid connection) placed inside the building.

19 of the analysed cases have installed photovoltaics for electricity production. In the following a few examples are described.

Villa Castelli, is heated by a heat pump and it was a requirement that the remaining energy demand should be covered locally and in a renewable way. Therefore, a PV-system, integrated in the roofing and not visible from outside, was installed. Before the heritage authorities approved the PV system, several prototypes were developed for a roof-integrated and preferably invisible installation. The heritage authorities opted for the doublecurled aluminium sheet covering of the roof with integrated mono-crystalline PV modules. The nominal power of the system is approx. $11 \mathrm{kWp}$. As a curiosity, it can be mentioned that a sailboat outfitter supplied the extra-thin PV modules.

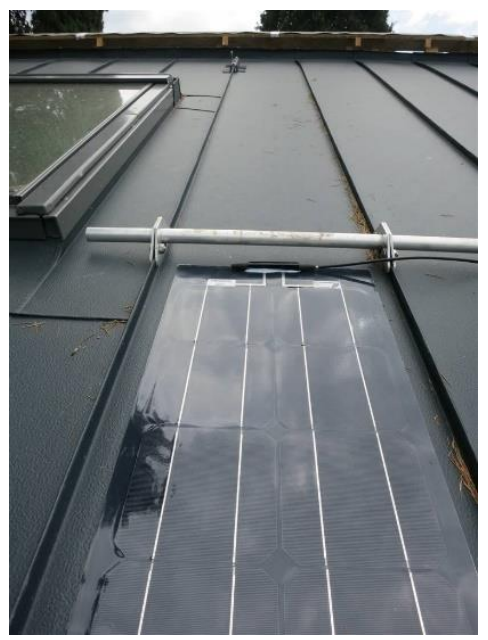

Figure 65: Villa Castelli. PV-panels integrated in the roof. 
In Doragno Castle, a PV-system was integrated in the south-east and south-west slopes of the roofs. As with the solar thermal installation, the selection of roofing materials allows for integrating the PV-system in order to make a uniform surface, i.e. the panels are coplanar to the roof.

In Solar Silo, there were no restrictions on the roof and façade of the building and therefore innovative coloured customized photovoltaic modules were used, creating a particular visual design to the building. As the "Gundeldinger Feld"-area as a whole is under heritage protection, the remodelled building was required to match the style and colour scheme of the site. The cladding (facade) consists of coloured frameless photovoltaic modules and fibre cement panels. The entire roof is covered with coloured and conventional PV modules. The modules are ventilated and serve as the water-bearing stratum. The total collector area is $159 \mathrm{~m}^{2}$ and the system has a nominal power of $24 \mathrm{kWp}$.

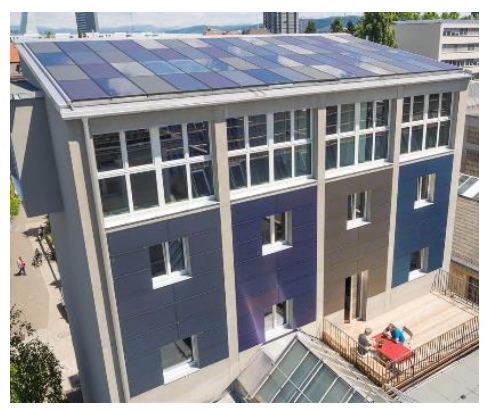

Figure 66: Solar Silo. Coloured customized photovoltaic modules creating a particular visual design.

In Platzbonhof, two photovoltaic systems of $19 \mathrm{kWp}$ were installed on the roof of the nearby stable. One system is used for power generation for the dwelling and the other for agricultural use, and power exchange occurs at both plants. This solution shows the utilization of the roof of a neighbouring building in order not to disturb the historic buildings.

Similar to Platzbonhof, in Ansitz Mairhof, a photovoltaic system was installed on a neighbouring building. The system was installed on the roof of the newly built carport. The carport and its roof surface are designed in such a way that the largest possible roof surface is created in order to make optimal use of the area. The carport stands at an elevation approx. $3 \mathrm{~m}$ above the level of the road. The inclination is chosen in such a way that the photovoltaic system is not visible from the street. The collector area is approx. $200 \mathrm{~m}^{2}$ and the system has a nominal power of $15.3 \mathrm{kWp}$.

\subsubsection{Geothermal (heat pump)}

Geothermal energy based on e.g. heat pumps generally come in two different variants, i.e. horizontal pipes or vertical boreholes. For both solutions the basic principle is that a heat transfer medium (typically brine) is circulated through the loop collecting heat from the ground and via a heat pump the temperature is raised and the energy is utilized in the heating system for heating, domestic hot water or most often both. Horizontal pipes can be challenging since it requires a relatively large area to implement and vertical boreholes are not allowed everywhere.

In the analysed cases there are 10 examples utilizing geothermal energy. Five of these are briefly described in the following.

In Villa Castelli a brine-water heat pump uses geothermal energy to generate energy via three deep probes that go down to $80 \mathrm{~m}$ depth each. The heat pump supplies both heating, domestic hot water and air conditioning for the building. It must be emphasized that the specific power of cooling is very low (soft cooling) in order to avoid condensation in the floor structure. The domestic hot water tank is 900 litres and can therefore store quite a lot of thermal energy. The nominal power of the heat pump is $14 \mathrm{~kW}$ and heating/cooling is supplied through radiating floor system.

In Ansitz Kofler the air for the ventilation system is pre-heated in winter and pre-cooled in summer with a groundto-air "heat exchanger". The pipes are placed at 1.5 to $2.0 \mathrm{~m}$ depth in the soil around the buildings, with a gradient of $2 \%$ to guarantee condensation discharge and an antimicrobial layer consisting of silver particles. The air inlet has a dust and pollen filter, a further benefit to allergy sufferers. The system does not rely on a heat pump as other systems and instead the inlet-air is simply cooled/heated by travelling through pipes in the ground. 


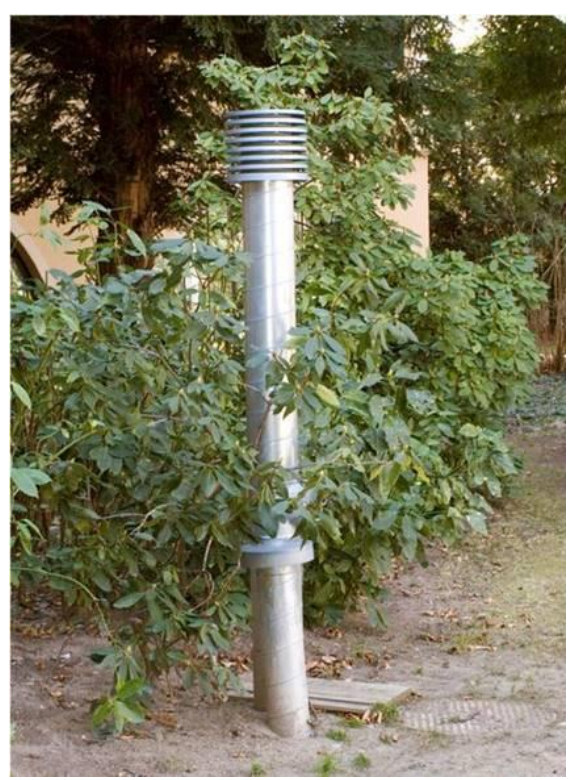

Figure 67: Ansitz Kofler. Inlet for the ventilation system pre-heating the air via the ground.

For Basilica di Santa Maria di Collemaggio (a church) heating is supplied directly to the benches where people sit during the service. It is a hydronic heating system coupled with ground-source heat pumps, and combines good local comfort levels and significant energy savings and low or no impact on the artworks and building structures. The radiant plates are extruded aluminium profiles, specifically adapted for the purpose and they have internal ducts in which the heat transfer fluid flows. The wooden structure of the bench is designed to fully integrate all pipes and joints. The heat pump has a nominal power of $30 \mathrm{~kW}$ (brine input temperature of $0^{\circ} \mathrm{C}$ and water output temperature at $40^{\circ} \mathrm{C}$ ) with a seasonal coefficient of performance (SCOP) of the geothermal heat pump system equal to 4.0 .

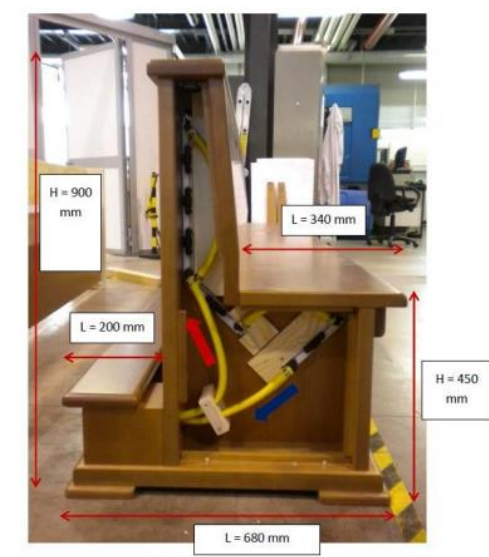

Figure 68: Basilica di Santa Maria di Collemaggio.

Cross-section of bench with hydronic heating.

In Bauernhof Trins a heat pump was installed. The heat pump is centrally located in the basement and bore holes go down to $100 \mathrm{~m}$. The heat pump does not influence the historical value of the building.

For Mercado del Val three reversible ground to water geothermal heat pumps were installed, getting temperature from vertical boreholes done on the ground (42 boreholes of $120 \mathrm{~m}$ ). In order to cover the energy needs a low temperature heating and cooling system was selected, with radiant floor on the ground floor and first floor and fan 
coils in the basement. For the DHW supply, the geothermal heat pumps will be supported by storage tanks with electric immersion heaters to prevent legionella. The installation does not affect the conservation of the building as the geothermal system is placed in the basement.

Ground source heat pump solutions are sensible solutions in historic buildings since the installation is usually not visible and therefore does not intervene with the heritage values of the building.

\subsubsection{Biomass}

Using biomass (e.g. pellet boilers) as a primary heating source is a solution typically adopted in areas where there is no possibility for connecting to e.g. district heating, gas or similar. As secondary heating (e.g. using wood stoves) biomass is used in living rooms where people spend most of the time. Some modern (low energy) homes also have wood stoves, but then it is usually for the sake of comfort or creating a certain atmosphere in the house.

25 of the analysed cases utilise biomass as a renewable energy source, and in the following some examples are described.

In Ansitz Kofler a pellet boiler was installed. The pellet boiler covers heating and domestic hot water production for now, however (as mentioned above) it is foreseen that the pellet boiler will be complemented by solar thermal in the future.

In Bauernhof Trins a tiled stove is used as secondary heating source. The stove is placed in the most frequently used room (the living room). It was built in the original Tyrolean style with tiles and is integrated in the wall to increase the thermal mass.

Maison Rubens uses a wood stove for secondary heating. No further information is available.

In Aussergrubhof a wood chip plant was installed. No further information is available.

In Platzbonhof the heating system is fired with wood chips and $120 \mathrm{~m}^{3}$ are consumed per year. The plant is placed in the basement and can only be reached via an outside staircase. The plant supplies domestic hot water and heating for the house, as well as hot water for the barn (milking machine cleaning and cattle troughs). The wood from the surrounding forests is used and the ecological footprint is low.

Ansitz Mairhof used to be oil heated, but the increased energy efficiency of the building achieved through the renovation, made it possible for a shift to log wood heating. The wood is obtained locally from a forest that is part of the property.

For Timber-framed house in Alsace a $25 \mathrm{~kW}$ pellet boiler was installed in the technical room together with a pellet silo. It provides heating and domestic hot water through two storage tanks. A traditional stove, a so-called "Kachelofe" was also installed as additional heating source. This kind of stove was the principal heating system in Alsatian houses before the 20th century and it is supplied by logs and takes its air supply directly from the outside.

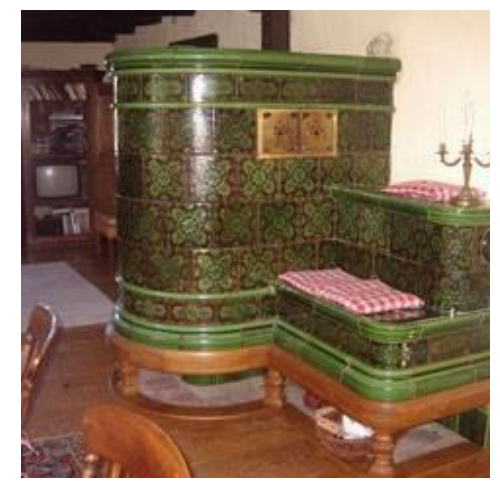

Figure 69: Timber-framed house in Alsace. Traditional stove as extra heating source.

In Rainhof the new heating system works as a combination of a biogas boiler and wood-chip boiler providing heat and domestic hot water. A traditional stove is kept in the "Stube" (living room) although it is not heated with firewood anymore; instead, it is connected to the central boiler. Almost all rooms are heated through a floor 
heating system. However, in some rooms old wood floorboards were restored and here therefore radiators are used instead. The biomass boiler uses wood chip produced directly from the farm woodland and stored in the silo next to the building. The gas boiler runs with the biogas produced from the manure of the cattle reared in the farm in a biogas plant built between the stable and residential building. The energy produced from biogas and wood chips cover the energy need of the Rainhof, a nearby house (included holiday apartments) and the stable.

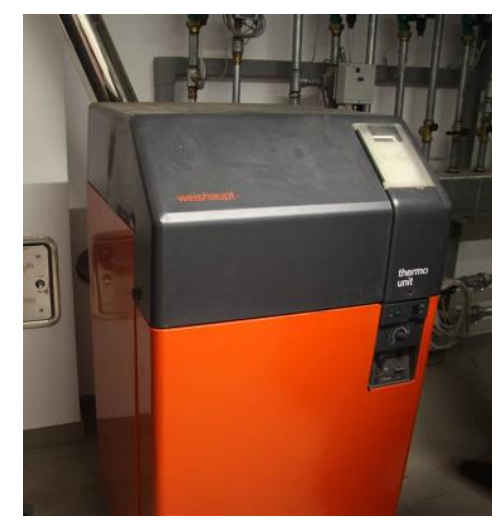

Figure 70: Rainhof. Biomass boiler that together with a biogas boiler produces heat and hot water. 


\section{Evaluation / Outcomes}

Lastly, the evaluation section presents the results of the intervention in terms of energy efficiency improvement, internal climate control, financial assessment, and environmental impact. This section, however, presents more detailed information and might only be relevant for users looking for a deeper understanding of the project. Thus, most of the information is not displayed initially when accessing the site and it is only visible when extending the collapsible menus (Figure 71).

This section is subdivided in four parts:

- Energy efficiency: summary of the building's post-retrofit energy performance. Opportunity to include detailed energy data from calculations, monitoring or real consumption.

- Internal climate: considerations on the effect of the post-retrofit internal climate on users' comfort, users' energy behaviour and artefact conservation.

- Costs: detailed information of the financial aspects of the retrofit. It offers the opportunity to break down investment and running costs showing the eventual financial result.

- Environment: overview of the environmental aspects of the intervention: life cycle assessment, water, indoor air quality, and transport and mobility.

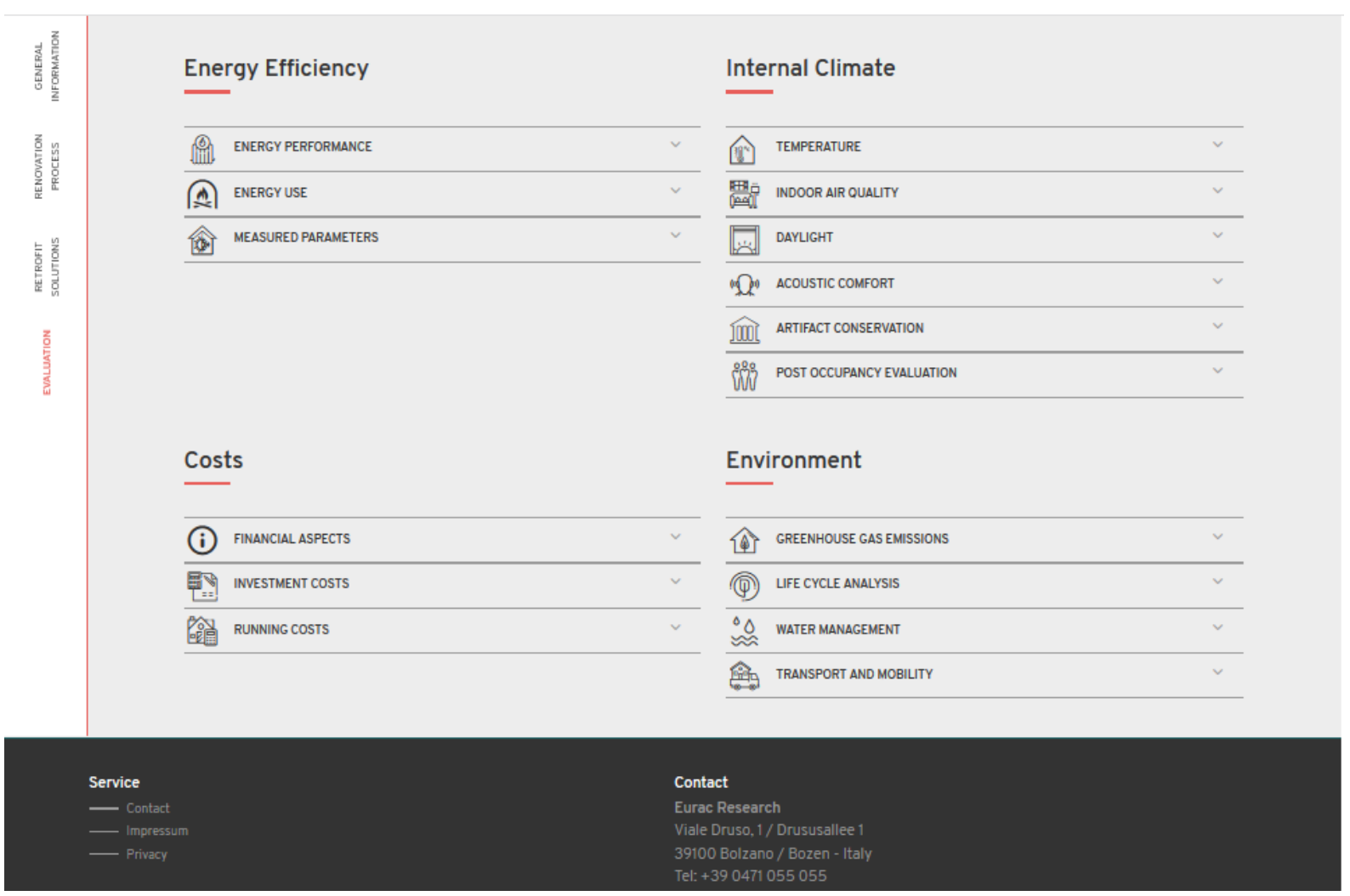

Figure 71: Evaluation section as presented in the site www.hiberatlas.com

\subsection{Energy efficiency}

The energy efficiency subsection looks at different aspects of the building's performance differentiating between Energy Performance Certificates, heating energy use, primary energy use, and the monitored performance.

\subsubsection{Energy performance}

Of the 69 cases studies included in this report, 51 (74\% of cases) have an Energy Performance Certificate (EPC) (Table 16). The methodologies, as well as the thresholds between classes, change between countries and therefore 
a comparison based on the energy classes could be misleading if not handled appropriately 4 . This information, however, can be very useful for building owners when looking at buildings in the same region of their own property. A further analysis of the energy classes can only be performed when the number of EPC per country reaches a significant sample. At the moment, the EPC distribution per country is as follows: Austria $14(87.5 \%$ of documented cases), Belgium 2 (100\%), Switzerland $3(30 \%)$, Germany $6(85.7 \%)$, Denmark $1(33.3 \%)$, France $3(100 \%)$, Italy 15 (93.8\%), Slovenia 3 (75\%), Spain 1 (50\%), Turkey 0, UK 3 (100\%), USA 0.

Regarding the distribution of EPC according to the main use of the building, the results were as follows: Residential (urban) 12 cases (75.0\%), Residential (rural) 23 (79.3\%), Offices 0, Educational \& Research 3 (75.0\%), Wholesale \& Retail 2 (100.0\%), Hotel \& Restaurant 2 (100.0\%), Religious 0, Other 10 (90.9\%).

In addition to this information, any voluntary certification (such as LEED, BREEAM, etc.) was also documented. Of the 69 documented cases studies, 16 have some sort of voluntary certification (23\%). Most of these certificates have a regional or national scope. For instance, different standards of Minergie are found among the Swiss cases, whereas two buildings in France were certified to obtain the "Bâtiment basse consummation" label. Other regional certificates found in the sample are "Gründerzeit mit Zukunft”, "Klimaaktiv“, and „Kommunaler Gebäudeausweis“ in Austria, „Plus-Wärmeenergiehaus“ in Switzerland, „KfW-Effizienzhaus“ in Germany, or "KlimaHaus/CasaClima“ in Italy. Two of the most well-known standards at international level, EnerPhit and LEED, are only found in two cases, one in Austria and one in the USA respectively.

Table 17: Availability of EPC and calculation methods

\begin{tabular}{|c|c|c|c|c|c|}
\hline No & Case study & 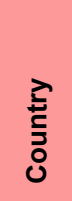 & 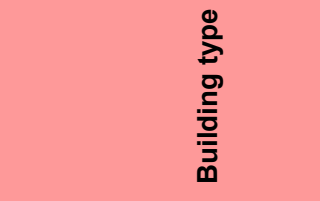 & U & 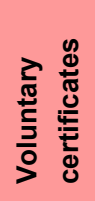 \\
\hline 1 & Klostergebäude Kaiserstrasse & AT & Residential (urban) & 1 & 1 \\
\hline 2 & $\underline{\text { Farmhouse Trins }}$ & AT & Residential (rural) & 0 & 1 \\
\hline 3 & Hof 6, Schwarzenberg, Voralberg & AT & Residential (rural) & 0 & 0 \\
\hline 4 & Kelchalm - Bochumer alpine hut & AT & Hotel \& Restaurant & 1 & 1 \\
\hline 5 & Mariahilferstrasse 182 & AT & Residential (urban) & 1 & 0 \\
\hline 6 & House Maurer, Wolfurt & AT & Residential (rural) & 1 & 0 \\
\hline 7 & House Breuer, Tschagguns & AT & Residential (rural) & 1 & 0 \\
\hline 8 & Music school in Velden & AT & Educational \& Research & 1 & 1 \\
\hline 9 & Hof Neuhäusl & AT & Residential (rural) & 1 & 0 \\
\hline 10 & Community Hall Zwischenwasser & AT & Other & 1 & 1 \\
\hline 11 & Freihof Sulz & AT & Other & 1 & 0 \\
\hline 12 & Oeconomy building Josef Weiss & AT & Other & 1 & 0 \\
\hline 13 & Giatla Haus & AT & Hotel \& Restaurant & 1 & 0 \\
\hline 14 & Rhine Valley House Irgang & AT & Residential (rural) & 1 & 0 \\
\hline 15 & Baur Residence, Lustenau & AT & Residential (rural) & 1 & 0 \\
\hline 16 & Kasperhof & AT & Residential (rural) & 1 & 0 \\
\hline 17 & Maison Rubens & $\mathrm{BE}$ & Residential (urban) & 1 & 0 \\
\hline 18 & Half-timberframed house in Alken & $\mathrm{BE}$ & Residential (rural) & 1 & 0 \\
\hline 19 & Doragno Castle, Rovio & $\mathrm{CH}$ & Residential (rural) & 0 & 0 \\
\hline 20 & $\underline{\text { Solar silo }}$ & $\mathrm{CH}$ & Offices & 0 & 0 \\
\hline 21 & Wohn- und Geschäftshaus Feldbergstrasse & $\mathrm{CH}$ & Residential (urban) & 0 & 1 \\
\hline 22 & Mehrfamilienhaus Magnusstrasse & $\mathrm{CH}$ & Residential (urban) & 0 & 1 \\
\hline 23 & $\underline{\text { St. Franziskus Church Ebmatingen }}$ & $\mathrm{CH}$ & Religious & 0 & 0 \\
\hline 24 & Kindergarten and apartments (PEB) Chur & $\mathrm{CH}$ & Other & 0 & 0 \\
\hline 25 & Single family home Luisenstrasse - Bern & $\mathrm{CH}$ & Residential (urban) & 1 & 1 \\
\hline 26 & $\underline{\text { Single Family House - Gstaad }}$ & $\mathrm{CH}$ & Residential (rural) & 1 & 0 \\
\hline 27 & Glaserhaus in Affoltern & $\mathrm{CH}$ & Residential (rural) & 0 & 1 \\
\hline
\end{tabular}

\footnotetext{
${ }^{4}$ For more information on EPC and Historic buildings see "Energy Efficiency and Historic Buildings: Energy Performance Certificates" Historic England May 2015. Available at https://historicengland.org.uk/imagesbooks/publications/eehb-energy-performance-certificates/heag015-eehb-epcs/
} 


\begin{tabular}{|c|c|c|c|c|c|}
\hline 28 & PalaCinema Locarno & $\mathrm{CH}$ & Other & 1 & 1 \\
\hline 29 & Casa Rossa Chemnitz & $\mathrm{DE}$ & Residential (urban) & 1 & 0 \\
\hline 30 & Rathaus Bergrheinfeld & $\mathrm{DE}$ & Other & 1 & 0 \\
\hline 31 & Farmhouse Straub & $\mathrm{DE}$ & Residential (rural) & 1 & 1 \\
\hline 32 & Early work Sep Ruf & $\mathrm{DE}$ & Residential (rural) & 1 & 0 \\
\hline 33 & Ackerbürgerhäuschen & $\mathrm{DE}$ & Residential (rural) & 1 & 0 \\
\hline 34 & Ritterhof & $\mathrm{DE}$ & Residential (rural) & 0 & 1 \\
\hline 35 & Rathaus Burgkunstadt & $\mathrm{DE}$ & Other & 1 & 0 \\
\hline 36 & Osramhuset (The Osram Building) & DK & Other & 1 & 0 \\
\hline 37 & Klitgaarden & DK & Residential (rural) & 0 & 0 \\
\hline 38 & Ryesgade 30 & DK & Residential (urban) & 0 & 0 \\
\hline 39 & Timber-framed house in Alsace & $\mathrm{FR}$ & Residential (rural) & 1 & 1 \\
\hline 40 & Elementary School in Mulhouse & FR & Educational \& Research & 1 & 1 \\
\hline 41 & Timber-framed barn in the north of France & FR & Residential (rural) & 1 & 0 \\
\hline 42 & $\underline{\text { Rainhof }}$ & IT & Residential (rural) & 1 & 0 \\
\hline 43 & Villa Castelli & IT & Residential (rural) & 1 & 0 \\
\hline 44 & Ansitz Kofler & IT & Residential (urban) & 1 & 1 \\
\hline 45 & Basilica di Santa Maria di Collemaggio & IT & Religious & 0 & 0 \\
\hline 46 & House Pernter & IT & Residential (rural) & 1 & 0 \\
\hline 47 & Kohlerhaus & IT & Residential (urban) & 1 & 0 \\
\hline 48 & Ruckenzaunerhof & IT & Residential (rural) & 1 & 0 \\
\hline 49 & Aussergrubhof & IT & Other & 1 & 0 \\
\hline 50 & Oberbergerhof & IT & Residential (rural) & 1 & 0 \\
\hline 51 & Platzbonhof & IT & Residential (rural) & 1 & 0 \\
\hline 52 & Mairhof & IT & Residential (rural) & 1 & 0 \\
\hline 53 & $\underline{\text { Obergasserhof }}$ & IT & Residential (rural) & 1 & 0 \\
\hline 54 & Rebecco Farm & IT & Other & 1 & 0 \\
\hline 55 & Villa Capodivacca & IT & Residential (urban) & 1 & 0 \\
\hline 56 & House Moroder & IT & Residential (urban) & 1 & 0 \\
\hline 57 & Huberhof & IT & Residential (rural) & 1 & 0 \\
\hline 58 & Notarjeva vila & SL & Residential (urban) & 1 & 0 \\
\hline 59 & Rožna ulica 15 , Idrija & $\mathrm{SL}$ & Residential (urban) & 1 & 0 \\
\hline 60 & Hiša trentarskih vodnikov & $\mathrm{SL}$ & Other & 1 & 0 \\
\hline 61 & Idrija mercury smelting plant & $\mathrm{SL}$ & Educational \& Research & 0 & 0 \\
\hline 62 & Mercado del Val, Valladolid & $S P$ & Wholesafe \& Retail & 1 & 0 \\
\hline 63 & Correria 119 & $\mathrm{SP}$ & Residential (urban) & 0 & 0 \\
\hline 64 & Ahmet Aga Mansion & TK & Offices & 0 & 0 \\
\hline 65 & Necip Pasa Library & TK & Educational \& Research & 0 & 0 \\
\hline 66 & Downie's Cottage & UK & Residential (rural) & 1 & 0 \\
\hline 67 & Hollyrood Park Lodge & UK & Wholesafe \& Retail & 1 & 0 \\
\hline 68 & Annat Road & UK & Residential (urban) & 1 & 0 \\
\hline \multirow[t]{2}{*}{69} & Aspinall Courthouse & USA & Offices & 0 & 1 \\
\hline & Total & & & 51 & 16 \\
\hline
\end{tabular}

\subsubsection{Energy use}

Information about energy use was gathered to differentiate between energy demand for heating (plus Domestic Hot Water if relevant) and total primary energy (being the amount of energy that must be generated originally in order to meet the total energy demand of a building from renewable and non-renewable energy sources). For each category, the data pre and post intervention is gathered (as well as information about whether or not DHW in included in the calculation).

It is important to highlight that just 14 cases are fully documented, but also that only in four cases no information about energy was reported. In general, the information varies notably between cases, making the comparison across the sample challenging. 
The average energy demand was around $215 \mathrm{kWh} / \mathrm{m}^{2} \mathrm{y}$ (SD 109) before the intervention and $68 \mathrm{kWh} / \mathrm{m}^{2} \mathrm{y}$ (SD 68) afterwards (Table 17). Although these data should treated be carefully, since it includes different methodologies, climatic conditions, and very different building typologies, a first analysis indicates a total energy reduction of around $70 \%$.

This result can only be considered useful when the number of projects documented, including the evaluation section, forms for a statistically significant sample that allows considering the effect of the different variables listed above. In general, and as it can see below, the samples in each subset is in some cases still limited making the analysis difficult.

In addition to this information, the calculation method used for the energy performance assessment was also documented. When compiling a case study, authors can choose from six different options. NA, Steady-state simulation, Dynamic simulation, On-site monitoring, Derived from energy bills, and Other ${ }^{5}$.

Of the 35 cases documented, the majority (21) used steady-state simulations (e.g. PHPP or national tools) while only in two cases a dynamic simulation was used. In two cases the energy use was measured on site whereas in one case it was derived from energy bills. In four cases the answer was "Other".

Table 18: Energy demand and primary energy demand before and after renovation

\begin{tabular}{|c|c|c|c|c|c|c|c|c|c|}
\hline \multirow[t]{2}{*}{ No } & \multirow[t]{2}{*}{ Case study } & \multirow[t]{2}{*}{ 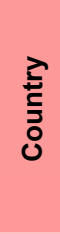 } & \multirow[t]{2}{*}{$\begin{array}{l}\text { ס } \\
\stackrel{+}{ \pm} \\
\Sigma\end{array}$} & \multicolumn{3}{|c|}{ 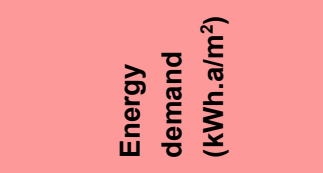 } & \multicolumn{3}{|c|}{ 胥 } \\
\hline & & & & DHW & Before & After & DHW & Before & After \\
\hline 1 & Klostergebäude & AT & monitoring & 1 & & 75.8 & 0 & & 125.0 \\
\hline 2 & Farmhouse Trins & AT & steady state & 1 & & 59.5 & 1 & & 15.8 \\
\hline 3 & Hof 6, Schwarzenberg. & AT & steady state & 1 & & 73.0 & 0 & & \\
\hline 4 & Kelchalm - Bochumer hut & AT & & 1 & 145.0 & 38.0 & 1 & & 241.8 \\
\hline 5 & Mariahilferstrasse 182 & AT & & 1 & & 27.7 & & & 92.3 \\
\hline 6 & House Maurer, Wolfurt & AT & & 0 & 142.0 & 35.0 & 1 & 65.1 & 48.1 \\
\hline 7 & House Breuer, Tschagguns & AT & steady state & 0 & & 33.0 & 0 & & \\
\hline 8 & Music school in Velden & AT & steady state & 0 & 418.1 & 27.2 & 1 & 856.2 & 247.4 \\
\hline 9 & $\underline{\text { Hof Neuhäusl }}$ & AT & & 0 & & 17.0 & 0 & & 17.0 \\
\hline 10 & Community Hall & AT & & 1 & 68.1 & 32.3 & 0 & & \\
\hline 11 & Freihof Sulz & AT & & 0 & 167.0 & 55.8 & 0 & & \\
\hline 12 & Oeconomy building Weiss & AT & NA & 1 & & 71.0 & 1 & & 90.2 \\
\hline 13 & Giatla Haus & AT & & 1 & & 65.4 & 0 & & \\
\hline 14 & Rhine Valley House Irgang & AT & & 0 & & 45.0 & 0 & & \\
\hline 15 & Baur Residence, Lustenau & AT & & 0 & 28.8 & 38.0 & 0 & & 7.6 \\
\hline 16 & Kasperhof & AT & & 0 & & 33.1 & 0 & & 107.6 \\
\hline 17 & Maison Rubens & $\mathrm{BE}$ & steady state & 1 & & 45.0 & 1 & & 71.0 \\
\hline 18 & Half-timber framed in Alken & $\mathrm{BE}$ & steady state & 1 & 358.4 & & 1 & 591.3 & \\
\hline 19 & Doragno Castle, Rovio & $\mathrm{CH}$ & steady state & 1 & & 45.6 & 0 & & \\
\hline 20 & $\underline{\text { Solar silo }}$ & $\mathrm{CH}$ & SIA 380/1:2009 & 1 & 96.6 & 66.0 & 0 & & \\
\hline 21 & Wohnhaus Feldbergstrasse & $\mathrm{CH}$ & steady state & 1 & 190.0 & 35.0 & 1 & 210.0 & 50.0 \\
\hline 22 & Magnusstrasse & $\mathrm{CH}$ & & 1 & & 46.8 & 0 & & \\
\hline 23 & St. Franziskus Church & $\mathrm{CH}$ & steady state & 1 & 78.9 & 51.1 & 1 & 109.3 & 87.0 \\
\hline 24 & Kindergarten Chur & $\mathrm{CH}$ & steady state & 1 & 250.0 & 40.0 & 1 & 310.0 & 75.0 \\
\hline 25 & Single family home - Bern & $\mathrm{CH}$ & steady state & 1 & 201.5 & 35.2 & 1 & 218.0 & 51.7 \\
\hline 26 & Single House - Gstaad & $\mathrm{CH}$ & steady state & 1 & & 31.2 & 1 & & 109.9 \\
\hline 27 & Glaserhaus in Affoltern & $\mathrm{CH}$ & monitoring & 1 & 182.7 & 26.6 & 1 & 277.3 & 79.1 \\
\hline 28 & PalaCinema Locarno & $\mathrm{CH}$ & Minergie & 1 & & 38.0 & 1 & & 38.0 \\
\hline
\end{tabular}

\footnotetext{
${ }^{5}$ For more information on Simulation Models and Energy Efficiency in Historic Buildings please see: https://historicengland.org.uk/whats-new/research/back-issues/simulation-models-and-energyefficiency-in-historic-buildings/
} 


\begin{tabular}{|c|c|c|c|c|c|c|c|c|c|}
\hline 29 & $\underline{\text { Casa Rossa Chemnitz }}$ & $\mathrm{DE}$ & & 1 & & 55.3 & 1 & & 62.8 \\
\hline 30 & Rathaus Bergrheinfeld & $\mathrm{DE}$ & & 0 & & 54.9 & 0 & 196.9 & 105.4 \\
\hline 31 & Farmhouse Straub & $\mathrm{DE}$ & steady state & 1 & & 47.3 & 1 & & 12.3 \\
\hline 32 & Early work Sep Ruf & $\mathrm{DE}$ & & 1 & 352.4 & 140.4 & 1 & 390.8 & 31.1 \\
\hline 33 & Ackerbürgerhäuschen & $\mathrm{DE}$ & & 1 & & 61.9 & 1 & & 85.1 \\
\hline 34 & $\underline{\text { Ritterhof }}$ & $\mathrm{DE}$ & from energy bills & 1 & & 60.0 & 1 & & 12.0 \\
\hline 35 & $\underline{\text { Rathaus Burgkunstadt }}$ & $\mathrm{DE}$ & & 1 & & 51.2 & 1 & & 112.5 \\
\hline 36 & Osramhuset & DK & NA & 1 & 158.0 & 37.0 & 0 & & \\
\hline 37 & $\underline{\text { Klitgaarden }}$ & DK & & 0 & & & 0 & & \\
\hline 38 & Ryesgade 30 & DK & steady state & 1 & 152.0 & 56.0 & 0 & & \\
\hline 39 & $\underline{\text { Timber-framed house }}$ & $\mathrm{FR}$ & steady state & 1 & 352.0 & 94.0 & 1 & 352.0 & 94.0 \\
\hline 40 & $\underline{\text { School in Mulhouse }}$ & FR & steady state & 1 & 214.0 & 69.0 & 1 & 214.0 & 69.0 \\
\hline 41 & Timber-framed barn & $\mathrm{FR}$ & & 1 & & 55.0 & & & \\
\hline 42 & Rainhof & IT & steady state & 0 & & 60.0 & 0 & & \\
\hline 43 & Villa Castelli & IT & steady state & 0 & 230.2 & 18.3 & 0 & & 32.4 \\
\hline 44 & Ansitz Kofler & IT & steady state & 0 & & 30.0 & 0 & & \\
\hline 45 & Basilica di Collemaggio & IT & NA & 0 & & 3.1 & 0 & 191.5 & 3.1 \\
\hline 46 & House Pernter & IT & NA & 1 & & 26.5 & & & \\
\hline 47 & $\underline{\text { Kohlerhaus }}$ & IT & & 1 & & 68.3 & 1 & & 16.9 \\
\hline 48 & Ruckenzaunerhof & IT & & 0 & 360.0 & 110.0 & 0 & & \\
\hline 49 & Aussergrubhof & IT & & 0 & & 107.0 & 1 & & 42.2 \\
\hline 50 & Oberbergerhof & IT & & 0 & 230.0 & 135.0 & 1 & 260.0 & 181.0 \\
\hline 51 & Platzbonhof & IT & steady state & 0 & & 74.0 & 1 & & 39.0 \\
\hline 52 & Mairhof & IT & NA & 1 & 283.0 & 66.0 & 1 & 283.0 & 57.0 \\
\hline 53 & $\underline{\text { Obergasserhof }}$ & IT & & 0 & & 99.0 & 1 & & 36.2 \\
\hline 54 & $\underline{\text { Rebecco Farm }}$ & IT & & 1 & & 89.3 & 1 & & \\
\hline 55 & Villa Capodivacca & IT & & 1 & 338.0 & 319.0 & 1 & & 361.9 \\
\hline 56 & House Moroder & IT & & 0 & 365.0 & 21.0 & 1 & 365.0 & 45.0 \\
\hline 57 & Huberhof & IT & & & 151.0 & 129.0 & & & \\
\hline 58 & Notarjeva vila & SL & steady state & 1 & & 28.0 & 1 & & 31.0 \\
\hline 59 & Rožna ulica 15 , Idrija & SL & & 0 & & 44.0 & & & 97.0 \\
\hline 60 & Hiša trentarskih vodnikov & SL & steady state & 1 & & 48.0 & & & 24.0 \\
\hline 61 & Idrija mercury smelting plant & SL & & 0 & & & & & \\
\hline 62 & Mercado del Val, Valladolid & $\mathrm{SP}$ & dynamic & 0 & 61.7 & 20.0 & 0 & 1300.2 & 326.9 \\
\hline 63 & $\underline{\text { Correria } 119}$ & $\mathrm{SP}$ & & 0 & & & & & \\
\hline 64 & Ahmet Aga Mansion & TK & & 1 & & 67.2 & 0 & & 67.2 \\
\hline 65 & Necip Pasa Library & TK & & 1 & & & 0 & & \\
\hline 66 & Downie's Cottage & UK & steady state & 1 & & 412.0 & 1 & 412.0 & 412.0 \\
\hline 67 & $\underline{\text { Hollyrood Park Lodge }}$ & UK & & 1 & & & 1 & 518.0 & 274.0 \\
\hline 68 & Annat Road & UK & & 1 & & 285.0 & 1 & & 285.0 \\
\hline \multirow[t]{2}{*}{69} & Aspinall Courthouse & USA & dynamic & 0 & & 75.8 & 1 & 134.4 & 66.2 \\
\hline & Average & & & 0.6 & 214.4 & 68.2 & 0.6 & 362.7 & \\
\hline
\end{tabular}

A summary of the energy use (for space heating) of the entire sample before and after the retrofits is presented in Figure 72. The results in the first plot on the left show graphically a significant reduction (around 70\%) in the demand of energy for space heating, but also a much more concentrated distribution of results. When looking at the histograms of both subsamples (before -middle- and after -right-), further detailed information can be accessed. After the renovation, most of the cases documented have an energy demand between 25 and $75 \mathrm{kWh} / \mathrm{m}^{2} \mathrm{y}$. 

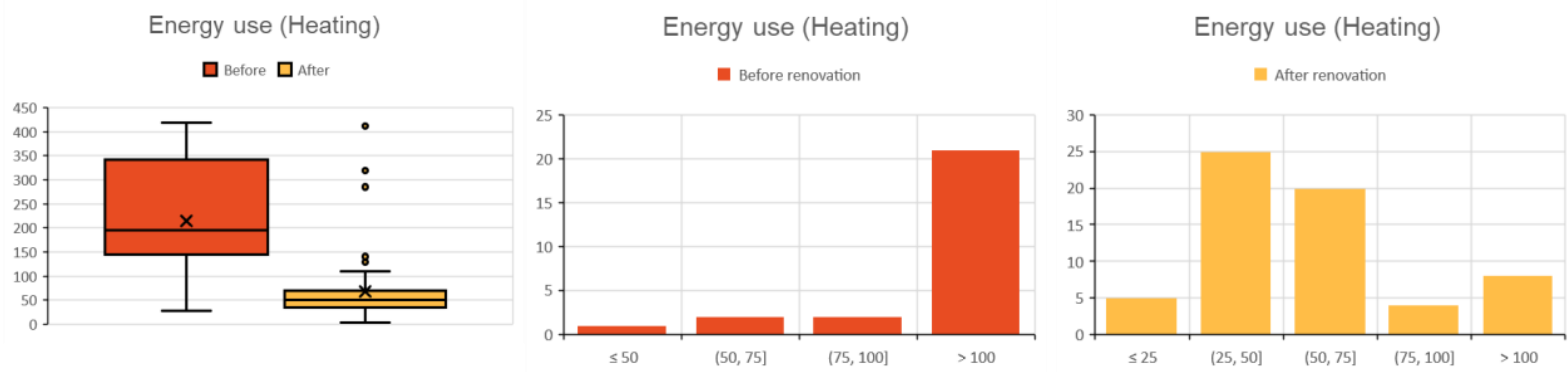

Figure 72. Energy use for space heating in $\mathrm{kWh} / \mathrm{m}^{2} \mathrm{y}$ before and after retrofit $\left(N_{\text {before }}=26, N_{\text {after }}=62\right)$

These results are heavily influenced by the climatic conditions, with colder climates having a higher energy demand. In order to minimise this uncertainty a detailed analysis of a subsample with homogeneous climatic conditions was performed. Of the documented cases, the climate $\mathrm{Cfb}^{6}$ (Temperate oceanic climate; coldest month averaging above $0{ }^{\circ} \mathrm{C}$, all months with average temperatures below $22^{\circ} \mathrm{C}$, and at least four months averaging above $10^{\circ} \mathrm{C}$ ) is by far the most representative (38 out of 69). The plots in Figure 73 show the same analysis for the subsample. The distribution of results of the case studies in climate $\mathrm{Cfb}$ is almost identical to that of the entire sample, with a great majority of cases above $100 \mathrm{kWh} / \mathrm{m}^{2} \mathrm{y}$ before the renovation and between 25 and $75 \mathrm{kWh} / \mathrm{m}^{2} \mathrm{y}$ afterwards.
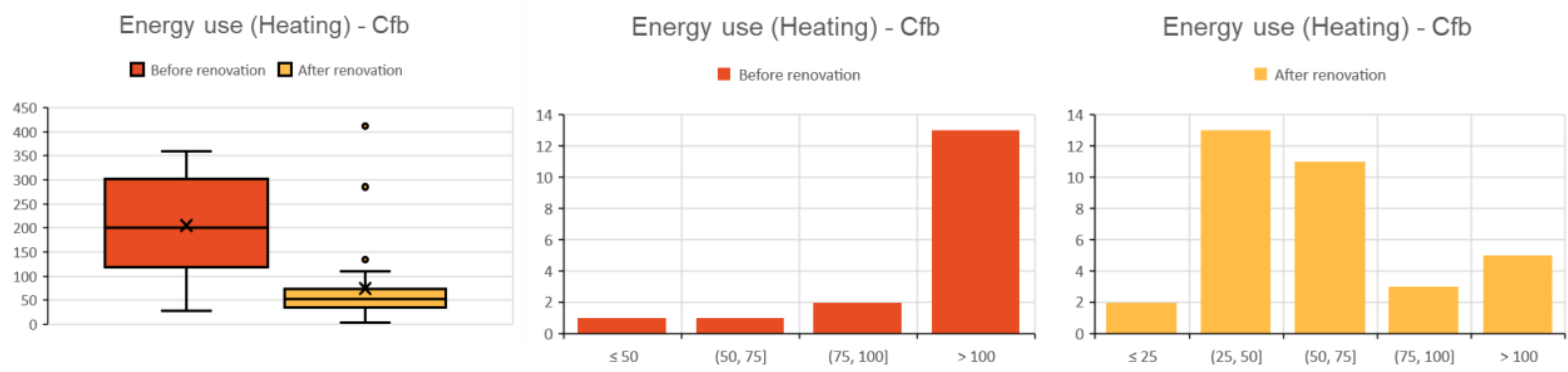

Figure 73. Energy use for space heating in $\mathrm{kWh} / \mathrm{m}^{2} . y$ before and after retrofit in climatic zone Cfb

The plots in Figure 74 show the energy savings achieved (in \%) when looking at the space heating demand. In all three plots, the energy savings are plotted as a function of a second variable (building construction period, Heating Degree Days, and Net Floor Area respectively). In general, the correlation between the energy savings and all three variables studied is fairly weak. Surprisingly, there is a positive relationship between savings and building constructions period suggesting that the more modern buildings profit more (in relative terms) of the energy retrofits. When it comes to HDD and NFA the relationship is slightly negative and positive respectively, suggesting that the colder the climate and the bigger the building, the higher is the energy saving achieved in relation to the energy demand before the intervention.

Energy savings (heating) / building age

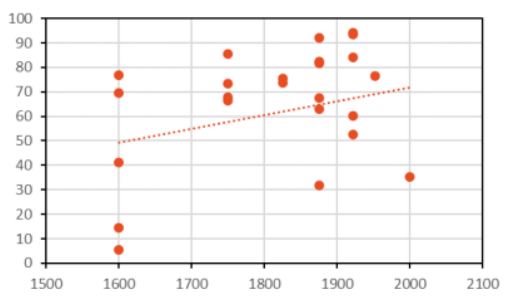

Energy savings (heating) / HDD

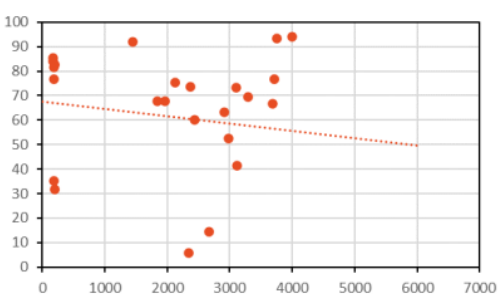

Energy savings (heating) / NFA

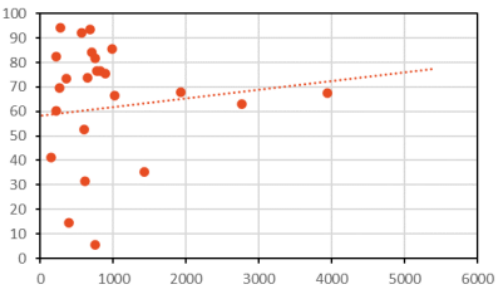

Figure 74. Energy savings (in \%) in terms of space heating demand $(\mathrm{N}=25)$ as a function of building age $\left(R^{2}=0.05\right)$, Heating Degree Days $\left(R^{2}=0,02\right)$, and Net Floor Area $\left(R^{2}=0.01\right)$.

The energy use for space heating (in $\mathrm{kWh} / \mathrm{m}^{2} \mathrm{y}$ ) and energy savings (in \%) is also studied according to the use of the building. In Figure 75, the results are filtered according to three different types of use (the most representative of the sample): residential (rural), residential (urban), and not residential. The histograms plotted below allow for a general comparison of the subsets even with different sample sizes. In all three cases, the majority of the cases lie within the 25 and $75 \mathrm{kWh} / \mathrm{m}^{2} \mathrm{y}$ and savings above $60 \%$, although with some small differences between the uses. In urban residential building the initial energy demand was the lowest, whereas the energy savings achieved lie mostly

\footnotetext{
${ }^{6}$ For more information on Köppen climate classification see: http://koeppen-geiger.vu-wien.ac.at/
} 
above $80 \%$. Rural residential buildings and non-residential building have a more comparative behaviour, with most cases distributed evenly between 25 and $75 \mathrm{kWh} / \mathrm{m}^{2} \mathrm{y}$ and an energy demand reduction of 60 to $80 \%$.

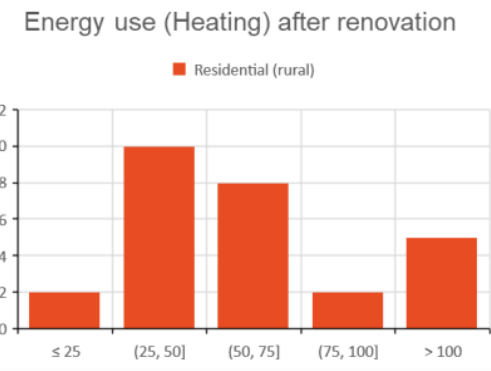

Savings (heating) after renovation

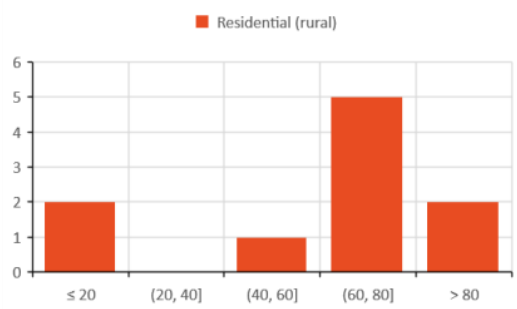

Energy use (Heating) after renovation In Residential (urban)

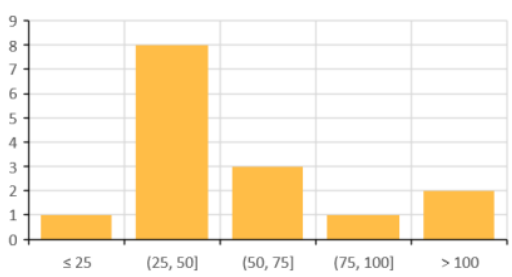

Savings (heating) after renovation

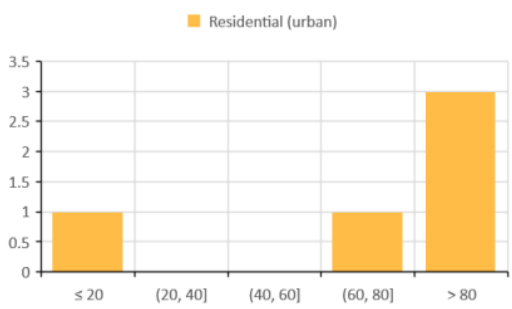

Energy use (Heating) after renovation

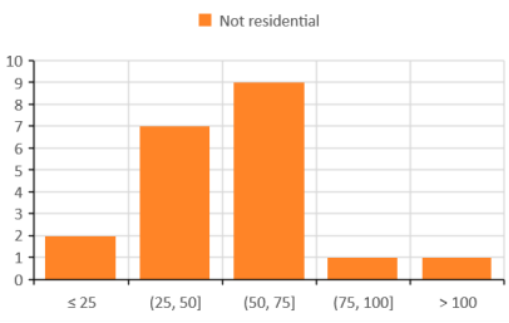

Savings (heating) after renovation

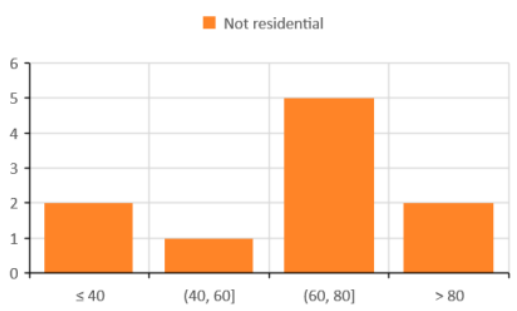

Figure 75. Energy use for space heating (in $\mathrm{kWh} / \mathrm{m}^{2} \mathrm{y}$ ) and energy savings (in \%) according to building use

To understand the limitations imposed by the legal status of the building (in terms of heritage protection), the analysis also looked into the different performance of listed and not listed buildings. Figure 76 shows the energy use and savings for both subsets. The energy use after the retrofit is slightly higher in listed buildings (top left). The histograms (top centre and top right) show slightly different curves with most cases in not listed buildings with an energy use between 25 and $50 \mathrm{kWh} / \mathrm{m}^{2} \mathrm{y}$ and 50 to $75 \mathrm{kWh} / \mathrm{m}^{2} \mathrm{y}$ in the case of listed buildings. The energy savings histograms on the other hand, show very different profiles. Whereas in the case of not listed building the cases are spread across the different ranges, in the case of listed buildings, most of the cases are concentrated in the range between 60 and $80 \%$ energy reduction.

Energy use (Heating) / Listed

口№ $\boldsymbol{\square}$ Yes

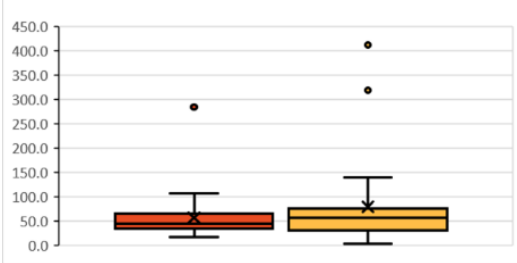

Energy use (Heating) after renovation

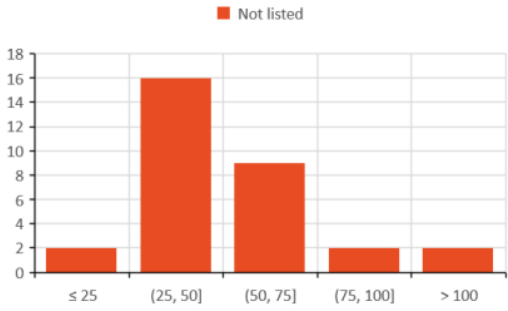

Savings (heating) after renovation

- Not listed

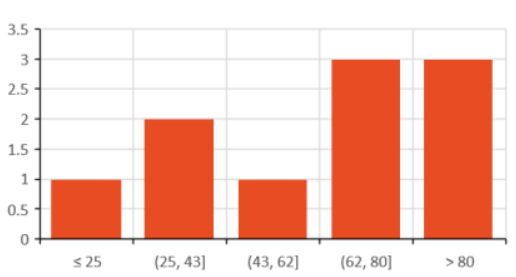

Energy use (Heating) after renovation

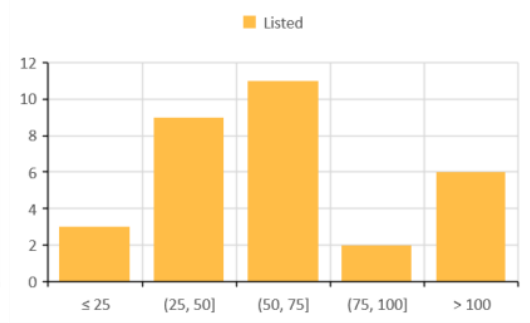

Savings (heating) after renovation

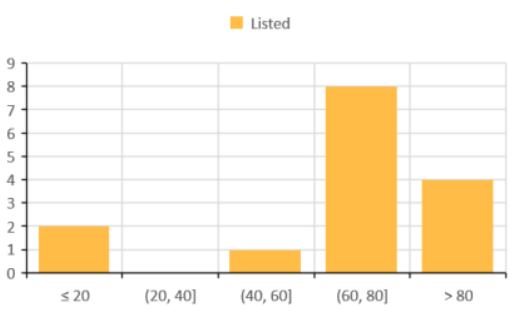

Figure 76. Energy use for space heating in $\mathrm{kWh} / \mathrm{m}^{2} \mathrm{y}$ and energy savings in \% for listed and not listed buildings

To understand how the relationship between energy consumption and other parameters of the building or site (building construction period, Heating Degree Days, and Net Floor Area), the energy use in $\mathrm{kWh} / \mathrm{m}^{2} \mathrm{y}$ is again plotted as a function of these three parameters in Figure 77. It is important to notice how in all three plots a similar trend can be observed. The dependency of all three variables decreases noticeably after the retrofit. That is, before the energy intervention the energy demand for space heating (per $\mathrm{m}^{2}$ ) is inversely proportional to the building age and net floor area while at the same time the correlation between energy use and HDD is positive. After the retrofit, the 
sign is maintained (negative for building age and area, and positive for HDD) but the dependency is much weaker and the variability between cases significantly lower.

Energy use (Heating) / building age

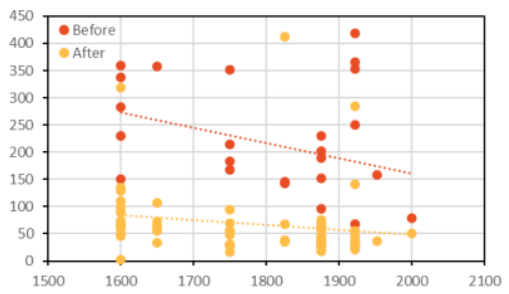

Energy use (Heating) / HDD

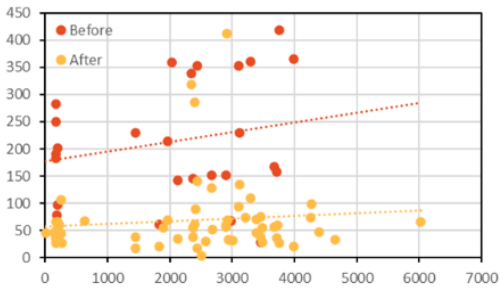

Energy use (Heating) / NFA

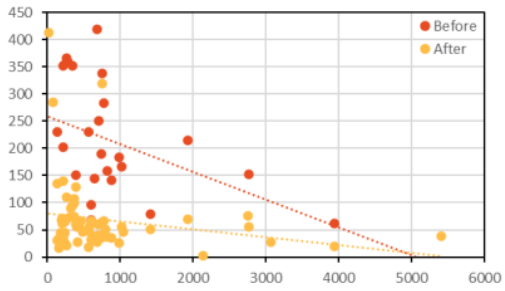

Figure 77. Energy use (in $\left.\mathrm{kWh} / \mathrm{m}^{2} \mathrm{y}\right)$ before and after retrofit as a function of building's age $\left(R^{2}\right.$ before $=0.11$, $R^{2}$ after=0.03), $H D D\left(R^{2}\right.$ before $=0.05, R^{2}$ after $\left.=0.01\right)$, and NFA $\left(R^{2}\right.$ before $=0.16, R^{2}$ after $\left.=0.04\right)$ (left to right)

In Figure 78, the effect of different parameters of the intervention on the energy use after the retrofit is studied in more detail. The plots below show (from left to right) the effect of the type of occupancy, insulation of the external wall, and use of mechanical ventilation with heat recovery (MVHR) on the final energy use for space heating. In general, no great differences can be observed when looking at the effect of a single variable with the exception perhaps of MVHR. Buildings without heat recovery (and that also includes cases relying exclusively on natural ventilation) present a much higher variability and overall higher energy use than those with MVHR. Surprisingly, when looking at the effect of wall insulation, the cases with no intervention present better results. However, it is worth noticing than only 6 cases with no intervention in the external walls are included in the sample and thus the significance of the results might be limited.
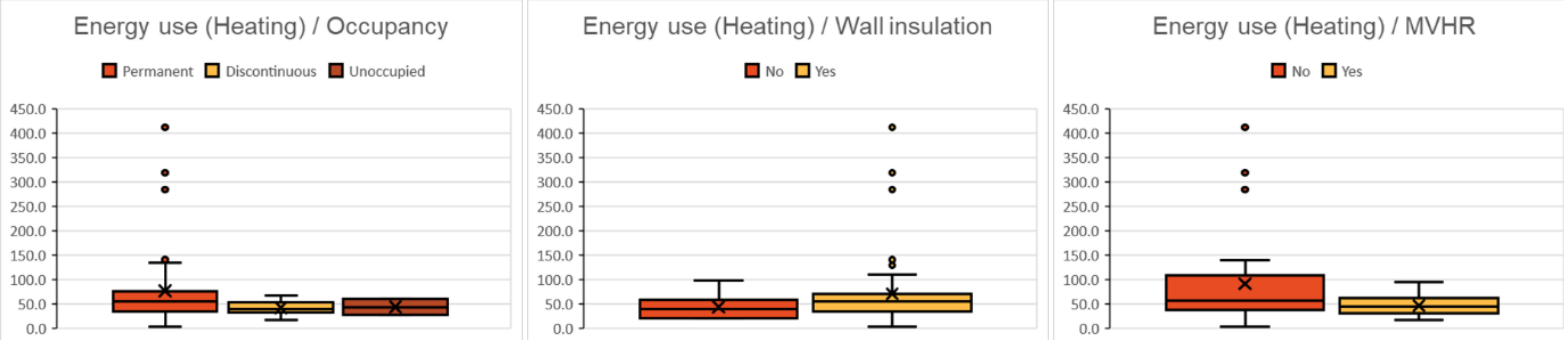

Figure 78. Energy use (in $\mathrm{kWh} / \mathrm{m}^{2} \mathrm{y}$ ) after retrofit according to different parameters of the intervention

Lastly, the effect of renewable energy systems is presented in Figure 79. In this case, and to take into consideration the contribution to the total energy performance of the building, the primary energy use of the case studies after the retrofit (in $\mathrm{kWh} / \mathrm{m}^{2} \mathrm{y}$ ) is studied in cases with and without Solar thermal (left) and PV systems (right). The positive effect of solar energy is even more evident in the case of photovoltaic systems, where the total primary energy use is reduced from 113.6 to $56.1 \mathrm{kWh} / \mathrm{m}^{2} \mathrm{y}$.

Primary energy use / Solar thermal
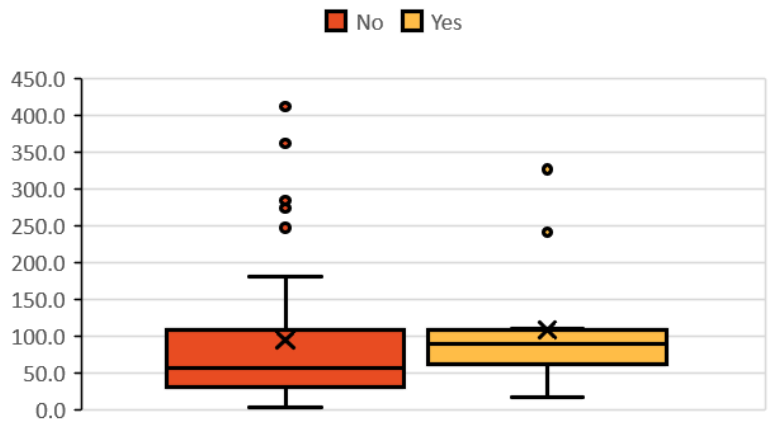

Primary energy use / PV
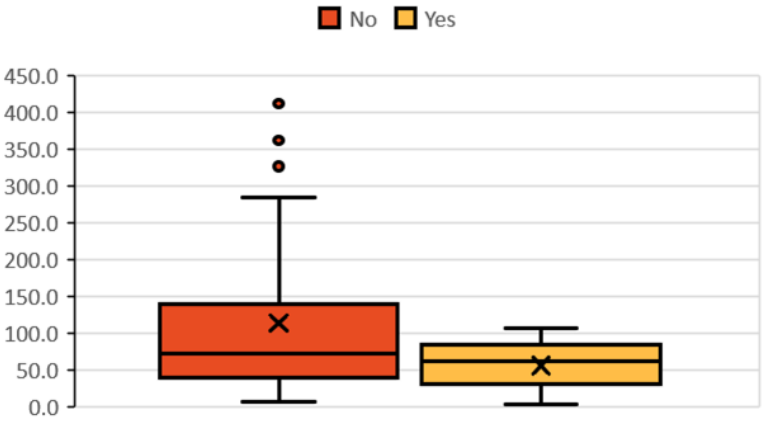

Figure 79. Total primary energy use (in $\mathrm{kWh} / \mathrm{m} 2 \mathrm{y}$ ) in cases with and without Solar thermal and PV systems 


\subsubsection{Measured parameters}

Regarding the acquisition of data directly from the cases studies (see Table 18), in 23 cases (33\% of the projects) the internal climate was monitored in some way (this includes short- and long-term monitoring), although only in 11 $(16 \%)$ of those the external climate was also monitored.

The hygrothermal performance of the intervention was monitored in 15 cases (22\% of the projects) and the energy behaviour of the users was recorded in five of the cases documented so far (7\%).

Unfortunately, in the majority of the cases the data monitored has not been made available as part of the documentation of the case studies.

Table 19: Overview of measured parameters

\begin{tabular}{|c|c|c|c|c|c|c|}
\hline No & Case study & 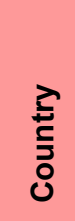 & 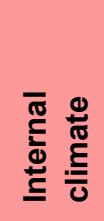 & 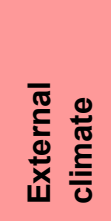 & 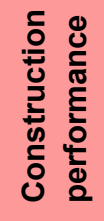 & ذِ \\
\hline 1 & Klostergebäude Kaiserstrasse & AT & 1 & 0 & 1 & 0 \\
\hline 2 & Farmhouse Trins & AT & 0 & 0 & 0 & 0 \\
\hline 3 & Hof 6. Schwarzenberg, Voralberg & AT & 0 & 0 & 0 & 0 \\
\hline 4 & Kelchalm - Bochumer alpine hut & AT & 0 & 0 & 0 & 0 \\
\hline 5 & Mariahilferstrasse 182 & AT & 1 & 1 & 0 & 0 \\
\hline 6 & House Maurer, Wolfurt & AT & 1 & 0 & 0 & 0 \\
\hline 7 & House Breuer, Tschagguns & AT & 0 & 0 & 0 & 0 \\
\hline 8 & Music school in Velden & AT & 1 & 1 & 0 & 0 \\
\hline 9 & Hof Neuhäusl & AT & 0 & 0 & 0 & 0 \\
\hline 10 & Community Hall Zwischenwasser & AT & 0 & 0 & 0 & 0 \\
\hline 11 & Freihof Sulz & AT & 0 & 0 & 1 & 0 \\
\hline 12 & Oeconomy building Josef Weiss & AT & 1 & 0 & 0 & 0 \\
\hline 13 & Giatla Haus & AT & 0 & 0 & 0 & 0 \\
\hline 14 & Rhine Valley House Irgang & AT & 0 & 0 & 0 & 1 \\
\hline 15 & Baur Residence, Lustenau & AT & 0 & 0 & 0 & 0 \\
\hline 16 & Kasperhof & AT & 0 & 0 & 0 & 0 \\
\hline 17 & Maison Rubens & $\mathrm{BE}$ & 0 & 0 & 1 & 0 \\
\hline 18 & Half-timberframed house in Alken & $\mathrm{BE}$ & 0 & 0 & 1 & 0 \\
\hline 19 & Doragno Castle, Rovio & $\mathrm{CH}$ & 0 & 0 & 0 & 0 \\
\hline 20 & $\underline{\text { Solar silo }}$ & $\mathrm{CH}$ & 0 & 0 & 0 & 0 \\
\hline 21 & Wohnhaus Feldbergstrasse & $\mathrm{CH}$ & 1 & 0 & 0 & 0 \\
\hline 22 & Mehrfamilienhaus Magnusstrasse & $\mathrm{CH}$ & 0 & 0 & 0 & 0 \\
\hline 23 & St. Franziskus Church Ebmatingen & $\mathrm{CH}$ & 0 & 0 & 0 & 0 \\
\hline 24 & Kindergarten and apartments Chur & $\mathrm{CH}$ & 1 & 1 & 1 & 0 \\
\hline 25 & Single family home Luisenstrasse - Bern & $\mathrm{CH}$ & 1 & 1 & 1 & 1 \\
\hline 26 & Single Family House - Gstaad & $\mathrm{CH}$ & 0 & 0 & 0 & 0 \\
\hline 27 & Glaserhaus in Affoltern & $\mathrm{CH}$ & 1 & 0 & 1 & 0 \\
\hline 28 & PalaCinema Locarno & $\mathrm{CH}$ & 0 & 0 & 0 & 0 \\
\hline 29 & $\underline{\text { Casa Rossa Chemnitz }}$ & $\mathrm{DE}$ & 1 & 0 & 0 & 0 \\
\hline 30 & $\underline{\text { Rathaus Bergrheinfeld }}$ & $\mathrm{DE}$ & 0 & 0 & 0 & 0 \\
\hline 31 & Farmhouse Straub & $\mathrm{DE}$ & 0 & 0 & 0 & 0 \\
\hline 32 & Early work Sep Ruf & $\mathrm{DE}$ & 0 & 0 & 0 & 0 \\
\hline 33 & Ackerbürgerhäuschen & $\mathrm{DE}$ & 0 & 0 & 0 & 0 \\
\hline 34 & Ritterhof & $\mathrm{DE}$ & 0 & 0 & 0 & 0 \\
\hline 35 & Rathaus Burgkunstadt & $\mathrm{DE}$ & 0 & 0 & 0 & 0 \\
\hline 36 & Osramhuset (The Osram Building) & DK & 0 & 0 & 0 & 0 \\
\hline 37 & $\underline{\text { Klitgaarden }}$ & DK & 1 & 1 & 1 & 0 \\
\hline 38 & Ryesgade 30 & DK & 1 & 0 & 0 & 0 \\
\hline
\end{tabular}




\begin{tabular}{|l|l|l|l|l|l|l|}
\hline 39 & Timber-framed house in Alsace & FR & 0 & 0 & 0 & 0 \\
\hline 40 & Elementary School in Mulhouse & FR & 1 & 0 & 0 & 0 \\
\hline 41 & Timber-framed barn in north of France & FR & 0 & 0 & 0 & 0 \\
\hline 42 & Rainhof & IT & 0 & 0 & 0 & 0 \\
\hline 43 & Villa Castelli & IT & 0 & 0 & 0 & 0 \\
\hline 44 & Ansitz Kofler & IT & 1 & 1 & 1 & 0 \\
\hline 45 & Basilica di Santa Maria di Collemaggio & IT & 0 & 0 & 0 & 0 \\
\hline 46 & House Pernter & IT & 0 & 0 & 0 & 0 \\
\hline 47 & Kohlerhaus & IT & 1 & 1 & 1 & 1 \\
\hline 48 & Ruckenzaunerhof & IT & 0 & 0 & 0 & 0 \\
\hline 49 & Aussergrubhof & IT & 0 & 0 & 0 & 0 \\
\hline 50 & Oberbergerhof & IT & 0 & 0 & 0 & 1 \\
\hline 51 & Platzbonhof & IT & 0 & 0 & 0 & 0 \\
\hline 52 & Mairhof & IT & 0 & 0 & 0 & 0 \\
\hline 53 & Obergasserhof & IT & 0 & 0 & 0 & 0 \\
\hline 54 & Rebecco Farm & IT & 0 & 0 & 0 & 0 \\
\hline 55 & Villa Capodivacca & IT & 0 & 0 & 0 & 0 \\
\hline 56 & House Moroder & IT & 0 & 0 & 0 & 0 \\
\hline 57 & Huberhof & IT & 1 & 0 & 0 & 0 \\
\hline 58 & Notarjeva vila & SL & 0 & 0 & 0 & 1 \\
\hline 59 & Rožna ulica 15, Idrija & SL & 0 & 0 & 0 & 0 \\
\hline 60 & Hiša trentarskih vodnikov & SL & 1 & 0 & 0 & 0 \\
\hline 61 & Idrija mercury smelting plant & SL & 0 & 0 & 0 & 0 \\
\hline 62 & Mercado del Val, Valladolid & SP & 1 & 1 & 1 & 0 \\
\hline 63 & Correria 119 & SP & 1 & 0 & 0 & 0 \\
\hline 64 & Ahmet Aga Mansion & TK & 1 & 1 & 1 & 0 \\
\hline 65 & Necip Pasa Library & TK & 1 & 1 & 1 & 0 \\
\hline 66 & Downie's Cottage & UK & 0 & 0 & 0 & 0 \\
\hline 67 & Hollyrood Park Lodge & UK & 1 & 0 & 1 & 0 \\
\hline 68 & Annat Road & UK & 1 & 1 & 1 & 0 \\
\hline 69 & Aspinall Courthouse & USA & 0 & 0 & 0 & 0 \\
\hline & Total & & 23 & 11 & 15 & 5 \\
\hline & & & & & \\
\hline
\end{tabular}




\subsection{Internal climate}

The section dedicated to the internal climate gathers the insights of occupants and users of the buildings documented. That is, the information gathered in this section it is mostly data of qualitative nature. Since this report tries to gather all the information included in the database to give an overview of the database, the table below only indicates whether the different questions had been compiled (1) or not (0).

In addition to that, information about the availability of a Post-Occupancy Evaluation (POE) was gathered. Of the 69 cases documented (see Table 19), only seven had conducted some sort of POE.

Table 20: Information on internal climate conditions

\begin{tabular}{|c|c|c|c|c|c|c|c|c|}
\hline No & Case study & 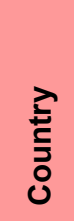 & 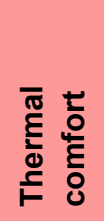 & $\underset{\mathbb{S}}{\mathbb{O}}$ & 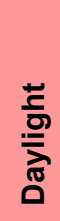 & 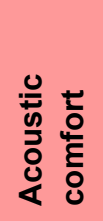 & 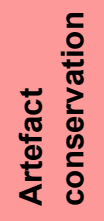 & 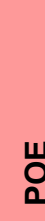 \\
\hline 1 & Klostergebäude Kaiserstrasse & AT & 1 & 0 & 0 & 0 & 0 & 0 \\
\hline 2 & Farmhouse Trins & AT & 1 & 1 & 0 & 0 & 0 & 0 \\
\hline 3 & Hof 6, Schwarzenberg, Voralberg & AT & 0 & 0 & 0 & 0 & 0 & 0 \\
\hline 4 & Kelchalm - Bochumer alpine hut & AT & 0 & 1 & 0 & 0 & 0 & 0 \\
\hline 5 & Mariahilferstrasse 182 & AT & 1 & 1 & 0 & 0 & 0 & 0 \\
\hline 6 & House Maurer, Wolfurt & AT & 0 & 0 & 1 & 1 & 0 & 0 \\
\hline 7 & House Breuer, Tschagguns & AT & 0 & 1 & 0 & 1 & 0 & 0 \\
\hline 8 & Music school in Velden & AT & 1 & 1 & 1 & 1 & 0 & 0 \\
\hline 9 & $\underline{\text { Hof Neuhäusl }}$ & AT & 1 & 1 & 1 & 1 & 0 & 0 \\
\hline 10 & Community Hall Zwischenwasser & AT & 1 & 1 & 0 & 1 & 0 & 0 \\
\hline 11 & Freihof Sulz & AT & 0 & 1 & 0 & 0 & 0 & 1 \\
\hline 12 & Oeconomy building Josef Weiss & AT & 1 & 1 & 1 & 1 & 0 & 0 \\
\hline 13 & Giatla Haus & AT & 0 & 0 & 0 & 0 & 0 & 0 \\
\hline 14 & Rhine Valley House Irgang & AT & 1 & 1 & 1 & 0 & 0 & 0 \\
\hline 15 & Baur Residence, Lustenau & AT & 0 & 0 & 0 & 0 & 0 & 0 \\
\hline 16 & Kasperhof & AT & 0 & 0 & 0 & 0 & 0 & 0 \\
\hline 17 & Maison Rubens & $\mathrm{BE}$ & 0 & 0 & 0 & 0 & 0 & 0 \\
\hline 18 & Half-timberframed house in Alken & $\mathrm{BE}$ & 1 & 1 & 1 & 1 & 0 & 0 \\
\hline 19 & Doragno Castle, Rovio & $\mathrm{CH}$ & 0 & 0 & 0 & 1 & 0 & 0 \\
\hline 20 & Solar silo & $\mathrm{CH}$ & 1 & 1 & 1 & 1 & 1 & 1 \\
\hline 21 & Wohnhaus Feldbergstrasse & $\mathrm{CH}$ & 1 & 1 & 1 & 1 & 0 & 0 \\
\hline 22 & Mehrfamilienhaus Magnusstrasse & $\mathrm{CH}$ & 1 & 1 & 1 & 1 & 0 & 0 \\
\hline 23 & St. Franziskus Church Ebmatingen & $\mathrm{CH}$ & 1 & 1 & 1 & 1 & 1 & 0 \\
\hline 24 & Kindergarten and apartments Chur & $\mathrm{CH}$ & 1 & 1 & 1 & 1 & 0 & 0 \\
\hline 25 & Single family home Luisenstrasse - Bern & $\mathrm{CH}$ & 1 & 1 & 1 & 1 & 0 & 0 \\
\hline 26 & Single Family House - Gstaad & $\mathrm{CH}$ & 1 & 1 & 1 & 1 & 0 & 0 \\
\hline 27 & Glaserhaus in Affoltern & $\mathrm{CH}$ & 1 & 1 & 1 & 1 & 1 & 0 \\
\hline 28 & PalaCinema Locarno & $\mathrm{CH}$ & 1 & 1 & 1 & 1 & 0 & 0 \\
\hline 29 & $\overline{\text { Casa Rossa Chemnitz }}$ & $\mathrm{DE}$ & 1 & 1 & 1 & 1 & 0 & 0 \\
\hline 30 & $\overline{\text { Rathaus Bergrheinfeld }}$ & $\mathrm{DE}$ & 0 & 0 & 0 & 0 & 0 & 0 \\
\hline 31 & Farmhouse Straub & $\mathrm{DE}$ & 1 & 1 & 1 & 1 & 0 & 0 \\
\hline 32 & Early work Sep Ruf & DE & 0 & 0 & 0 & 0 & 0 & 0 \\
\hline 33 & Ackerbürgerhäuschen & $\mathrm{DE}$ & 1 & 1 & 1 & 1 & 1 & 0 \\
\hline 34 & Ritterhof & $\mathrm{DE}$ & 1 & 1 & 0 & 1 & 0 & 0 \\
\hline 35 & $\overline{\text { Rathaus Burgkunstadt }}$ & $\mathrm{DE}$ & 0 & 0 & 0 & 1 & 0 & 0 \\
\hline 36 & Osramhuset (The Osram Building) & DK & 0 & 0 & 0 & 0 & 0 & 0 \\
\hline 37 & Klitgaarden & DK & 0 & 0 & 0 & 0 & 0 & 0 \\
\hline 38 & Ryesgade 30 & DK & 0 & 0 & 0 & 0 & 0 & 0 \\
\hline 39 & Timber-framed house in Alsace & $\mathrm{FR}$ & 1 & 0 & 1 & 0 & 0 & 0 \\
\hline
\end{tabular}




\begin{tabular}{|c|c|c|c|c|c|c|c|c|}
\hline 40 & Elementary School in Mulhouse & $\mathrm{FR}$ & 1 & 1 & 1 & 1 & 0 & 0 \\
\hline 41 & $\underline{\text { Timber-framed barn in north of France }}$ & FR & 1 & 0 & 0 & 0 & 0 & 0 \\
\hline 42 & Rainhof & IT & 1 & 1 & 1 & 1 & 0 & 0 \\
\hline 43 & Villa Castelli & IT & 1 & 0 & 0 & 0 & 0 & 1 \\
\hline 44 & Ansitz Kofler & IT & 1 & 0 & 1 & 0 & 0 & 1 \\
\hline 45 & Basilica di Santa Maria di Collemaggio & IT & 1 & 0 & 0 & 0 & 1 & 0 \\
\hline 46 & House Pernter & IT & 1 & 1 & 1 & 1 & 0 & 0 \\
\hline 47 & Kohlerhaus & IT & 1 & 1 & 1 & 1 & 1 & 0 \\
\hline 48 & $\underline{\text { Ruckenzaunerhof }}$ & IT & 1 & 0 & 1 & 0 & 0 & 0 \\
\hline 49 & Aussergrubhof & IT & 0 & 0 & 0 & 0 & 0 & 0 \\
\hline 50 & Oberbergerhof & IT & 1 & 0 & 0 & 0 & 0 & 0 \\
\hline 51 & Platzbonhof & IT & 1 & 1 & 1 & 1 & 0 & 0 \\
\hline 52 & Mairhof & IT & 1 & 1 & 0 & 0 & 1 & 0 \\
\hline 53 & Obergasserhof & IT & 1 & 1 & 1 & 0 & 0 & 0 \\
\hline 54 & Rebecco Farm & IT & 0 & 0 & 0 & 0 & 0 & 0 \\
\hline 55 & Villa Capodivacca & IT & 0 & 1 & 0 & 0 & 0 & 0 \\
\hline 56 & House Moroder & IT & 1 & 1 & 0 & 0 & 0 & 0 \\
\hline 57 & Huberhof & IT & 1 & 1 & 1 & 1 & 0 & 0 \\
\hline 58 & Notarjeva vila & SL & 0 & 0 & 0 & 0 & 0 & 0 \\
\hline 59 & Rožna ulica 15 , Idrija & SL & 0 & 0 & 0 & 0 & 0 & 0 \\
\hline 60 & Hiša trentarskih vodnikov & SL & 0 & 0 & 0 & 0 & 0 & 0 \\
\hline 61 & Idrija mercury smelting plant & SL & 0 & 0 & 0 & 0 & 0 & 0 \\
\hline 62 & Mercado del Val, Valladolid & SP & 1 & 1 & 1 & 1 & 0 & 0 \\
\hline 63 & $\underline{\text { Correria } 119}$ & SP & 0 & 0 & 0 & 0 & 0 & 0 \\
\hline 64 & Ahmet Aga Mansion & TK & 1 & 1 & 1 & 0 & 0 & 1 \\
\hline 65 & Necip Pasa Library & TK & 1 & 1 & 1 & 0 & 1 & 1 \\
\hline 66 & Downie's Cottage & UK & 1 & 1 & 1 & 0 & 1 & 0 \\
\hline 67 & Hollyrood Park Lodge & UK & 1 & 1 & 1 & 0 & 0 & 1 \\
\hline 68 & Annat Road & UK & 1 & 1 & 0 & 0 & 0 & 0 \\
\hline \multirow[t]{2}{*}{69} & Aspinall Courthouse & USA & 0 & 1 & 1 & 0 & 1 & 0 \\
\hline & Total & & 44 & 41 & 34 & 26 & 10 & 7 \\
\hline
\end{tabular}

\subsubsection{Temperature}

Occupants' opinion about the thermal comfort in the building after the renovation was documented in $44(64 \%)$ of the cases. This is a topic that is clearly comprehensible and appealing to the occupants of a building and therefore gathering information was relatively easy when compared to other categories. In general, the perception of thermal comfort after the intervention improved considerably.

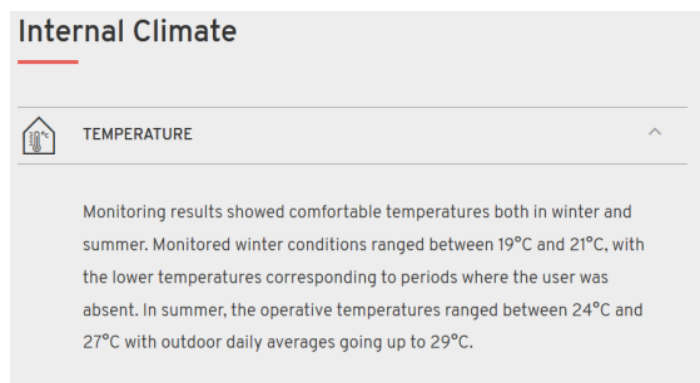

Figure 80: An example of the "Temperature" answers collected from the Ansitz Kofler case study

\subsubsection{Indoor Air Quality}

Occupants' opinion about the Indoor Air Quality (IAQ) in the building after the renovation was documented in 41 (59\%) of the cases. IAQ is also an easily comprehensible and appealing topic to users and occupants and therefore 
the collection of information was relatively successful (almost two thirds of the answers compiled). In general, the overall response was also positive in the case of IAQ.

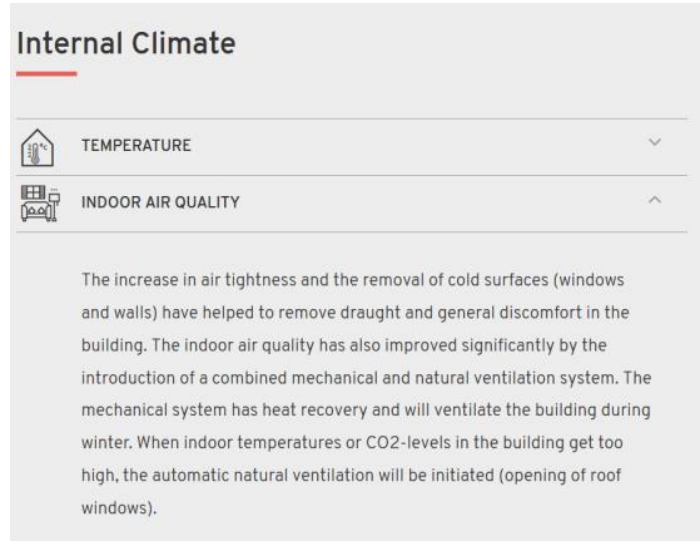

Figure 81. An example of the "IAQ" answers collected from the Osramhuset case study

\subsubsection{Daylight}

Occupants' opinion about the natural light in the building after the renovation was documented in 34 (49\%) of the cases. Analogously to IAQ, access to natural light is familiar to all and the rate of response also reached half the answers. In this case however, the information collected is rather superficial.

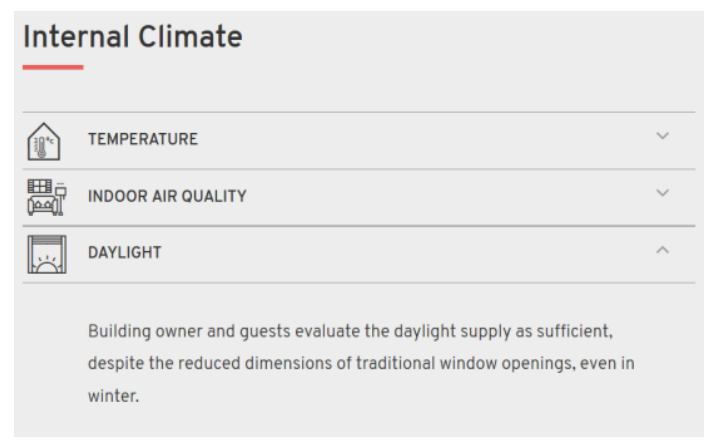

Figure 82. An example of the "Daylight" answers collected from the Rainhof case study

\subsubsection{Acoustic comfort}

Occupants' opinion about the acoustic comfort in the building after the renovation was documented in 26 of the cases. The evaluation of the acoustic performance of the intervention proved to be significantly more difficult to report and only $38 \%$ of the documented projects include some information about it. It is to be understood whether this is due to a lack of understanding of what the question was supposed to address or purely due to the lack of consideration of this topic during and after the renovation. 


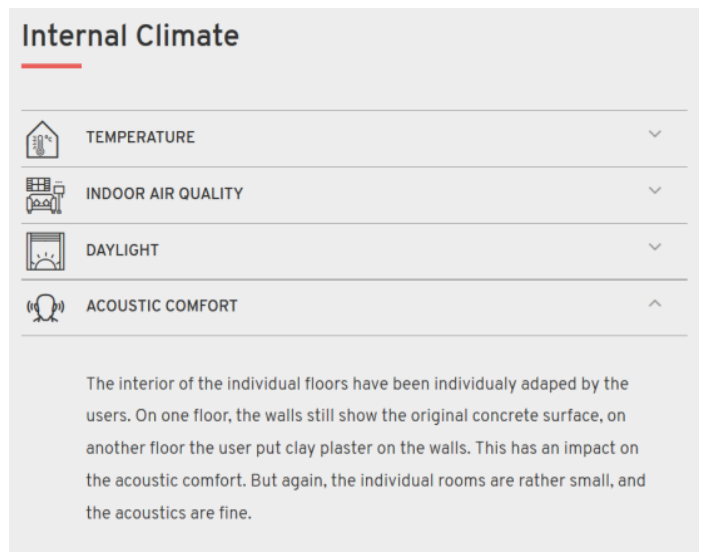

Figure 83. An example of the "Acoustic comfort" answers collected from the Solar silo case study

\subsubsection{Artefact conservation}

Occupants' opinion about the performance of the building in regard to artefact conservation was only documented in ten of the 69 cases. In line with acoustic comfort, only $15 \%$ of the cases reported information considering the impact of the renovation on the artefact conservation. Arguably, this can be due to the fact that most of the documented buildings are residential and do not have any historically significant artefacts that need especial attention.

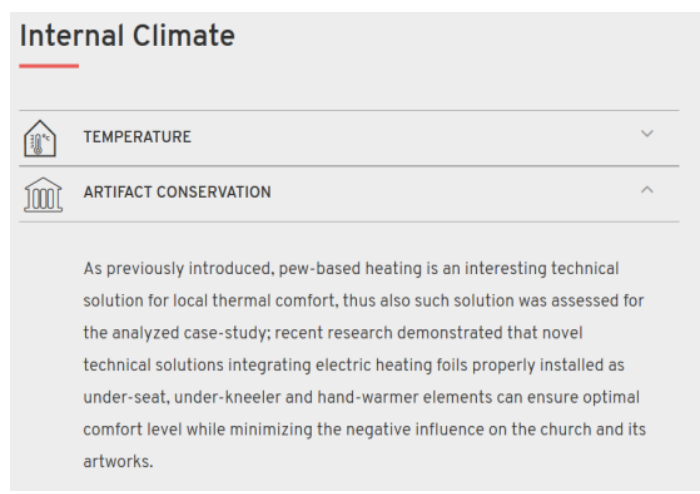

Figure 84. An example of the "Artefact conservation" answers collected from the Basilica di Santa Maria di Collemaggio 


\subsection{Costs}

In Table 20 a summary of the answers gathered to some of the most relevant questions about the financial aspects of the intervention is presented. For the sake of comparison, all information is reported in the same currency. Since most of the documented projects are in Europe, values are presented in Euros. Conversion rates (as for 18.05.2021) were used in case of other currencies $(1 \mathrm{USD}=0.82 €, 1 \mathrm{CHF}=0.91 €, 1 \mathrm{GBP}=1.16 €)$.

Table 21: Information on financial aspects and LCC

\begin{tabular}{|c|c|c|c|c|c|c|c|c|c|}
\hline \multirow[t]{2}{*}{ No } & \multirow[t]{2}{*}{ Case study } & \multirow[t]{2}{*}{ 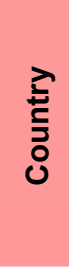 } & \multirow[t]{2}{*}{$\begin{array}{l}\frac{\bar{\pi}}{0} \\
\frac{\mathrm{C}}{\pi} \\
\frac{\mathrm{J}}{\mathrm{i}}\end{array}$} & \multirow[t]{2}{*}{ U. } & \multicolumn{2}{|c|}{ 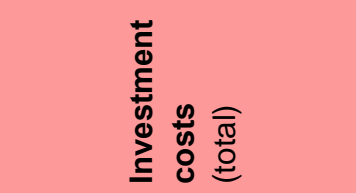 } & \multicolumn{3}{|c|}{ 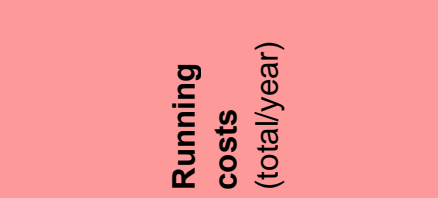 } \\
\hline & & & & & Total & Energy & Total & Heating & Electricity \\
\hline 1 & Klostergebäude & AT & 0 & 1 & $5,160,000$ & 912,000 & 31,570 & 20,900 & 10,670 \\
\hline 2 & Farmhouse Trins & AT & 1 & 0 & & & & & \\
\hline 3 & Hof 6, Schwarzenberg & AT & 0 & 0 & & & & & \\
\hline 4 & Kelchalm - Bochumer & AT & 0 & 0 & & & & & \\
\hline 5 & Mariahilferstrasse 182 & AT & 0 & 0 & $7,000,000$ & & & & \\
\hline 6 & House Maurer, Wolfurt & AT & 0 & 0 & & & 2,975 & & \\
\hline 7 & House Breuer & AT & 1 & 0 & 400,000 & & 1,400 & 400 & 1,000 \\
\hline 8 & Music school in Velden & AT & 1 & 0 & $1,460,826$ & & & & \\
\hline 9 & Hof Neuhäusl & AT & 0 & 0 & & & & & \\
\hline 10 & Community Hall & AT & 1 & 0 & $1,790,000$ & & & & \\
\hline 11 & Freihof Sulz & AT & 1 & 0 & & & & 4,000 & 200 \\
\hline 12 & Oeconomy building & AT & 0 & 0 & & & & & \\
\hline 13 & Giatla Haus & AT & 1 & 0 & & & & & \\
\hline 14 & Rhine Valley House & AT & 1 & 0 & & & & & \\
\hline 15 & Baur Residence & AT & 0 & 0 & & & & & \\
\hline 16 & Kasperhof & AT & 1 & 0 & $1,625,000$ & & & & \\
\hline 17 & Maison Rubens & $\mathrm{BE}$ & 1 & 0 & 281,060 & 75,000 & & & \\
\hline 18 & Half-timber framed & $\mathrm{BE}$ & 0 & 0 & & & & & \\
\hline 19 & Doragno Castle, Rovio & $\mathrm{CH}$ & 0 & 0 & & & & & \\
\hline 20 & Solar silo & $\mathrm{CH}$ & 1 & 0 & $1,092,000$ & $1,092,000$ & & & \\
\hline 21 & Feldbergstrasse & $\mathrm{CH}$ & 1 & 1 & $4,550,000$ & 455,000 & & & 145 \\
\hline 22 & Magnusstrasse & $\mathrm{CH}$ & 1 & 0 & $3,003,000$ & 646,100 & & & \\
\hline 23 & St. Franziskus Church & $\mathrm{CH}$ & 1 & 0 & $1,023,750$ & $1,023,750$ & 2,275 & 1,820 & 0 \\
\hline 24 & Kindergarten Chur & $\mathrm{CH}$ & 1 & 0 & $3,148,600$ & 127,400 & & & \\
\hline 25 & Single home - Bern & $\mathrm{CH}$ & 1 & 0 & & 182,000 & & & \\
\hline 26 & Single House, Gstaad & $\mathrm{CH}$ & 1 & 0 & & & & & \\
\hline 27 & Glaserhaus in Affoltern & $\mathrm{CH}$ & 1 & 0 & $2,548,000$ & 677,950 & 1,966 & 1,365 & 601 \\
\hline 28 & PalaCinema Locarno & $\mathrm{CH}$ & 1 & 0 & $30,630,600$ & & & & \\
\hline 29 & Casa Rossa Chemnitz & $\mathrm{DE}$ & 1 & 0 & & & & & \\
\hline 30 & Rathaus Bergrheinfeld & $\mathrm{DE}$ & 0 & 0 & 4.600 .000 & & & & \\
\hline 31 & Farmhouse Straub & $\mathrm{DE}$ & 1 & 0 & 931,684 & 528,333 & & & \\
\hline 32 & Early work Sep Ruf & DE & 0 & 0 & 387,972 & & & & \\
\hline 33 & Ackerbürgerhäuschen & $\mathrm{DE}$ & 1 & 0 & 549,460 & & & & \\
\hline 34 & Ritterhof & DE & 1 & 0 & & & & & \\
\hline 35 & Rathaus Burgkunstadt & $\mathrm{DE}$ & 1 & 0 & $3,000,000$ & & & & \\
\hline 36 & Osramhuset & DK & 0 & 0 & & & & & \\
\hline 37 & Klitgaarden & DK & 1 & 0 & & & & & \\
\hline 38 & Ryesgade 30 & DK & 0 & 0 & & & & & \\
\hline 39 & Timber-framed house & FR & 1 & 0 & & 150,000 & 2,500 & & \\
\hline 40 & School in Mulhouse & FR & 1 & 0 & $2,900,000$ & & & & \\
\hline
\end{tabular}




\begin{tabular}{|c|c|c|c|c|c|c|c|c|c|}
\hline 41 & Timber-framed barn & FR & 1 & 0 & & & & 2,000 & \\
\hline 42 & Rainhof & IT & 0 & 0 & & & & & 229 \\
\hline 43 & Villa Castelli & IT & 1 & 0 & $1,240,800$ & 321,480 & 1,800 & & \\
\hline 44 & Ansitz Kofler & IT & 1 & 0 & & & & & \\
\hline 45 & Basilica di Collemaggio & IT & 1 & 0 & 104,000 & & & & \\
\hline 46 & House Pernter & IT & 1 & 0 & 363,000 & & 2,100 & 600 & 1,500 \\
\hline 47 & Kohlerhaus & IT & 1 & 0 & & & & & \\
\hline 48 & Ruckenzaunerhof & IT & 0 & 0 & & & & & \\
\hline 49 & Aussergrubhof & IT & 0 & 0 & & & & & \\
\hline 50 & Oberbergerhof & IT & 1 & 0 & & & & & \\
\hline 51 & Platzbonhof & IT & 0 & 0 & & & & & \\
\hline 52 & Mairhof & IT & 1 & 0 & & & & & \\
\hline 53 & Obergasserhof & IT & 0 & 0 & & & & & \\
\hline 54 & Rebecco Farm & IT & 1 & 0 & 712,500 & & & & \\
\hline 55 & Villa Capodivacca & IT & 1 & 0 & & & & & \\
\hline 56 & House Moroder & IT & 1 & 0 & 500,000 & & 2,400 & & \\
\hline 57 & Huberhof & IT & 0 & 0 & & & & & \\
\hline 58 & Notarjeva vila & SL & 1 & 0 & 550,000 & & & & \\
\hline 59 & Rožna ulica 15 , Idrija & SL & 1 & 0 & 683,000 & & & & \\
\hline 60 & Hiša trentarskih & SL & 1 & 0 & & & & & \\
\hline 61 & Idrija mercury plant & SL & 1 & 0 & & & & & \\
\hline 62 & Mercado del Val & SP & 1 & 0 & $11,000,000$ & $2,000,000$ & 71,793 & & 71,793 \\
\hline 63 & Correria 119 & SP & 0 & 0 & & & & & \\
\hline 64 & Ahmet Aga Mansion & TK & 1 & 0 & & & & & \\
\hline 65 & Necip Pasa Library & TK & 1 & 0 & & & & & \\
\hline 66 & Downie's Cottage & UK & 1 & 0 & & 16,896 & 1,090 & & \\
\hline 67 & $\underline{\text { Hollyrood Park Lodge }}$ & UK & 1 & 0 & 76,494 & & 1,284 & 1,093 & \\
\hline 68 & Annat Road & UK & 1 & 0 & 19,728 & & & & \\
\hline \multirow[t]{2}{*}{69} & Aspinall Courthouse & USA & 1 & 1 & $12,300,000$ & & 18,229 & & \\
\hline & Total/Average & & 48 & 3 & $3,342,951 €$ & $586,279 €$ & $10,876 €$ & $4,022 €$ & $9,571 €$ \\
\hline
\end{tabular}

\subsubsection{Financial aspects}

Forty-eight $(69.5 \%)$ of the documented cases included in one way or another some considerations about the financial aspects of the project. As the next sections will show, the extent and depth of this information varies greatly across the cases.

It would be important to know whether the lack of response is due to difficulties in accessing the information or because cost was not issue considered explicitly during the retrofit.

Only three case studies (4\%) have included a Life Cycle Cost (LCC) assessment of the intervention.

\subsubsection{Investment costs}

Information regarding the investment (total or energy related) costs was included in 33 cases (48\%), but only in a third of them both considerations are included (11 cases).

The average investment of the interventions sums up to $3350 € / \mathrm{m}^{2}$, whereas the purely energy related costs are less than $600 € / \mathrm{m}^{2}$. That would represent that the energy efficiency improvement of a building represents only the $18 \%$ of the regular renovation cost. However, these results are to be considered carefully and due to the great variability between countries, a larger sample would be needed for a further analysis of the investment costs.

\subsubsection{Running costs}

Unfortunately, the information about the running costs of the retrofitted buildings is even more scarce. Only in 17 of the cases $(25 \%)$ the information was reported. The average total running cost for the retrofitted project was $10,876 €$ 
(11.6 $€ / \mathrm{m}^{2} \mathrm{a}$ ). When looking at different energy bills separately it was observed that the average yearly cost of heating was $4,022 €\left(5.0 € / \mathrm{m}^{2} \mathrm{y}\right)$ whereas the electricity bills account for $9,571 €\left(4.5 € / \mathrm{m}^{2} \mathrm{y}\right)$ per year on average. It is worth highlighting that the information is very partial and incomplete making the assessment and comparison very difficult.

\subsection{Environment}

The section dedicated to the environmental performance of the building gathered information of an extremely varied nature. Unfortunately, the cases documented so far (see Table 21) did not include a great amount of information and therefore the data presented here only shows whether the different sections had been compiled or not.

Table 22: Overview of environmental information (GHG, LCA, water and mobility)

\begin{tabular}{|c|c|c|c|c|c|c|}
\hline No & Case study & $\begin{array}{l}\stackrel{Z}{Z} \\
\stackrel{5}{J} \\
0\end{array}$ & 임 & త్ & 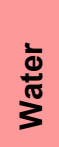 & 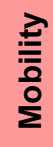 \\
\hline 1 & Klostergebäude Kaiserstrasse & AT & 0 & 0 & 0 & 0 \\
\hline 2 & Farmhouse Trins & AT & 0 & 0 & 0 & 0 \\
\hline 3 & Hof 6, Schwarzenberg, Voralberg & AT & 0 & 0 & 0 & 0 \\
\hline 4 & Kelchalm - Bochumer alpine hut & AT & 0 & 0 & 0 & 0 \\
\hline 5 & Mariahilferstrasse 182 & AT & 1 & 0 & 0 & 1 \\
\hline 6 & House Maurer, Wolfurt & AT & 0 & 0 & 0 & 0 \\
\hline 7 & House Breuer, Tschagguns & AT & 0 & 0 & 0 & 0 \\
\hline 8 & Music school in Velden & AT & 1 & 0 & 0 & 0 \\
\hline 9 & Hof Neuhäus| & AT & 0 & 0 & 0 & 0 \\
\hline 10 & Community Hall Zwischenwasser & AT & 0 & 1 & 0 & 0 \\
\hline 11 & Freihof Sulz & AT & 1 & 1 & 0 & 0 \\
\hline 12 & Oeconomy building Josef Weiss & AT & 0 & 0 & 0 & 0 \\
\hline 13 & Giatla Haus & AT & 0 & 0 & 0 & 0 \\
\hline 14 & Rhine Valley House Irgang & AT & 0 & 1 & 0 & 0 \\
\hline 15 & Baur Residence, Lustenau & AT & 0 & 0 & 0 & 0 \\
\hline 16 & Kasperhof & AT & 0 & 0 & 0 & 0 \\
\hline 17 & Maison Rubens & $\mathrm{BE}$ & 0 & 0 & 1 & 0 \\
\hline 18 & Half-timberframed house in Alken & $\mathrm{BE}$ & 0 & 0 & 0 & 0 \\
\hline 19 & $\overline{\text { Doragno Castle, Rovio }}$ & $\mathrm{CH}$ & 0 & 0 & 0 & 0 \\
\hline 20 & $\underline{\text { Solar silo }}$ & $\mathrm{CH}$ & 0 & 0 & 1 & 1 \\
\hline 21 & Wohnhaus Feldbergstrasse & $\mathrm{CH}$ & 1 & 1 & 0 & 0 \\
\hline 22 & Mehrfamilienhaus Magnusstrasse & $\mathrm{CH}$ & 1 & 0 & 0 & 0 \\
\hline 23 & St. Franziskus Church Ebmatingen & $\mathrm{CH}$ & 1 & 0 & 0 & 0 \\
\hline 24 & Kindergarten and apartments Chur & $\mathrm{CH}$ & 1 & 0 & 0 & 1 \\
\hline 25 & Single family home Luisenstrasse - Bern & $\mathrm{CH}$ & 0 & 0 & 1 & 0 \\
\hline 26 & Single Family House - Gstaad & $\mathrm{CH}$ & 0 & 0 & 0 & 0 \\
\hline 27 & Glaserhaus in Affoltern & $\mathrm{CH}$ & 0 & 0 & 1 & 1 \\
\hline 28 & PalaCinema Locarno & $\mathrm{CH}$ & 0 & 0 & 0 & 0 \\
\hline 29 & Casa Rossa Chemnitz & $\mathrm{DE}$ & 0 & 0 & 0 & 0 \\
\hline 30 & $\overline{\text { Rathaus Bergrheinfeld }}$ & $\mathrm{DE}$ & 0 & 0 & 0 & 0 \\
\hline 31 & Farmhouse Straub & $\mathrm{DE}$ & 0 & 0 & 0 & 0 \\
\hline 32 & Early work Sep Ruf & $\mathrm{DE}$ & 0 & 0 & 0 & 0 \\
\hline 33 & Ackerbürgerhäuschen & $\mathrm{DE}$ & 0 & 0 & 0 & 0 \\
\hline 34 & Ritterhof & $\mathrm{DE}$ & 0 & 0 & 0 & 0 \\
\hline 35 & Rathaus Burgkunstadt & $\mathrm{DE}$ & 0 & 0 & 0 & 0 \\
\hline 36 & Osramhuset (The Osram Building) & DK & 0 & 0 & 0 & 0 \\
\hline 37 & Klitgaarden & DK & 0 & 0 & 0 & 0 \\
\hline 38 & Ryesgade 30 & DK & 0 & 0 & 0 & 0 \\
\hline 39 & Timber-framed house in Alsace & $\mathrm{FR}$ & 0 & 0 & 0 & 0 \\
\hline
\end{tabular}




\begin{tabular}{|l|l|l|l|l|l|l|}
\hline 40 & Elementary School in Mulhouse & FR & 0 & 0 & 0 & 0 \\
\hline 41 & Timber-framed barn in north of France & FR & 0 & 0 & 0 & 0 \\
\hline 42 & Rainhof & IT & 0 & 0 & 0 & 0 \\
\hline 43 & Villa Castelli & IT & 0 & 0 & 0 & 0 \\
\hline 44 & Ansitz Kofler & IT & 0 & 0 & 1 & 1 \\
\hline 45 & Basilica di Santa Maria di Collemaggio & IT & 1 & 0 & 0 & 0 \\
\hline 46 & House Pernter & IT & 0 & 0 & 0 & 0 \\
\hline 47 & Kohlerhaus & IT & 0 & 0 & 0 & 0 \\
\hline 48 & Ruckenzaunerhof & IT & 0 & 0 & 0 & 0 \\
\hline 49 & Aussergrubhof & IT & 0 & 0 & 0 & 0 \\
\hline 50 & Oberbergerhof & IT & 1 & 0 & 0 & 0 \\
\hline 51 & Platzbonhof & IT & 1 & 0 & 0 & 0 \\
\hline 52 & Mairhof & IT & 0 & 0 & 0 & 0 \\
\hline 53 & Obergasserhof & IT & 1 & 0 & 0 & 0 \\
\hline 54 & Rebecco Farm & IT & 0 & 0 & 1 & 0 \\
\hline 55 & Villa Capodivacca & IT & 0 & 0 & 0 & 0 \\
\hline 56 & House Moroder & IT & 0 & 0 & 0 & 0 \\
\hline 57 & Huberhof & IT & 0 & 0 & 0 & 0 \\
\hline 58 & Notarjeva vila & SL & 0 & 0 & 0 & 0 \\
\hline 59 & Rožna ulica 15, Idrija & SL & 0 & 0 & 0 & 0 \\
\hline 60 & Hiša trentarskih vodnikov & SL & 1 & 0 & 0 & 0 \\
\hline 61 & Idrija mercury smelting plant & SL & 0 & 0 & 0 & 0 \\
\hline 62 & Mercado del Val, Valladolid & SP & 1 & 0 & 0 & 0 \\
\hline 63 & Correria 119 & SP & 0 & 0 & 0 & 0 \\
\hline 64 & Ahmet Aga Mansion & TK & 0 & 0 & 0 & 0 \\
\hline 65 & Necip Pasa Library & TK & 0 & 0 & 1 & 0 \\
\hline 66 & Downie's Cottage & UK & 1 & 0 & 0 & 0 \\
\hline 67 & Hollyrood Park Lodge & UK & 0 & 0 & 0 & 0 \\
\hline 68 & Annat Road & UK & 0 & 0 & 0 & 0 \\
\hline 69 & Aspinall Courthouse & & 14 & 4 & $\mathbf{8}$ & $\mathbf{5}$ \\
\hline & Total & & 0 & 0 & \\
\hline
\end{tabular}

\subsubsection{Greenhouse Gas emissions}

The greenhouse gas emissions of the buildings have been calculated in 14 of the cases (20\% of the documented projects). In many cases, GHG emissions are calculated as part of the EPC which. As the next sections will show, when there is no legal obligation, the environmental issues related to the retrofit are not considered, or at least not explicitly.

\subsubsection{Life Cycle Analysis}

Some life cycle assessment (LCA) has been conducted in four (less than 5\%) of the cases included in this report. However, the extent of these analysis varies greatly. For instance, in the case of the Community Hall in Zwischenwasser, only the LED lighting was considered in the assessment, whereas in Freihof Sulz or Rhine Valley House Irgang aspects related to primary energy content (non-renewable), Global Warming Potential and acidification associated to the new materials are calculated. It is worth noticing that three out of the four cases with LCA considerations are in Austria. 

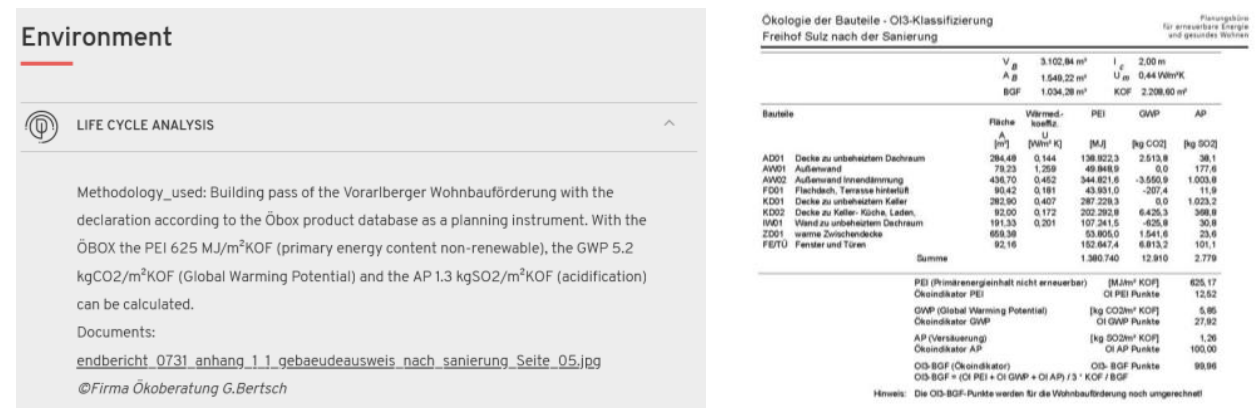

Figure 85. An example of the "Water management" answers collected from the Freihof Sulz case study

\subsubsection{Water Management}

Considerations about the water management of the building after the intervention were included in just eight cases $(11.6 \%$ of the projects) and the information included in these cases is usually rather generic. An example of this is shown in Figure 72.

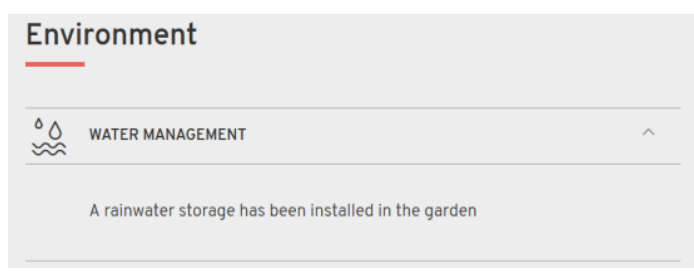

Figure 86. An example of the "Water management" answers collected from the Maison Rubens case study

\subsubsection{Transport and Mobility}

Considerations about transport and mobility after the intervention were included in five cases (7\%). It is worth noticing that the projects "Solar silo", "Glaserhaus in Affoltern" (both in Switzerland), and "Ansitz Kofler" (in Italy) included answers to both categories (Water management and Transport and mobility).

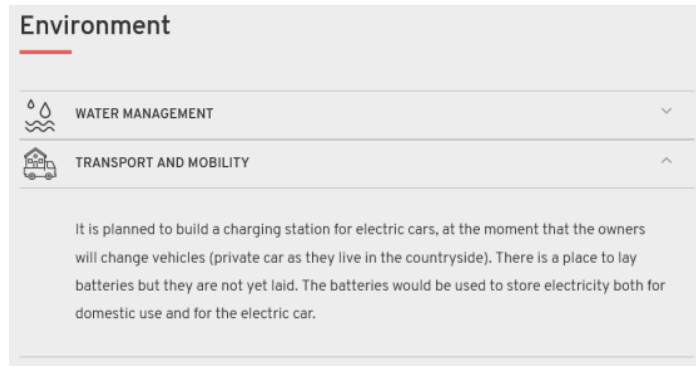

Figure 87. An example of the "Transport and Mobility" answers collected from the Glaserhaus case study 


\section{Favourable framework conditions}

When looking at possible case studies and good practice examples the project team agreed to set the "threshold" not in terms of fixed values for e.g. energy performance or energy reduction but to select cases representing solutions beyond business as usual (for the respective country).

In addition to the description of the technical solutions applied in the individual case studies, the focus was also on the description of the framework conditions that made the implementation of the projects possible in the first place.

Such favourable conditions could be, for example:

- Financial incentives

- Integration in research projects

- Cooperative planning process (owners, planners/architects, heritage authority)

- Fundamental change of use of buildings

As can easily be seen, these external conditions are very different factors that can be described individually, but only allow qualitative statements to be made regarding their incentive effect on the specific project.

In the context of this chapter, therefore, these different favourable framework conditions will be briefly described and it will be shown in which of the projects they have had a positive impact on the development.

\subsection{Financial incentives and financing models}

In order to stimulate the implementation of innovative projects that also set an example in terms of energy saving and climate protection, several states and/or regions have created financial incentive models that can be designed in different ways. Furthermore, some cases were implemented through private sponsoring or groups of private investors. In principle, the following models can be distinguished:

- Tax incentives

- Non-repayable grants

- Soft loans

- Private sponsoring (e.g. companies)

- Group of private investors

Criteria for obtaining public subsidies can be formulated, for example, in such a way that the achievement of a certain energy standard after renovation is required or that a minimum level of energy saving must be achieved through the measures implemented.

\subsection{Integration in research projects and programmes}

Some case studies have been implemented as part of a research project or national research programme, typically providing additional expertise and resources for the following possible actions:

- Detailed building survey and evaluation of the historical value of the building

- Integrated planning right from the start

- Feasibility studies concerning the technical measures

- Life cycle cost analysis

- Monitoring concept for energy (heating, cooling, yield generated from renewables etc.), comfort parameters (temperature, humidity etc.)

- Detailed technical monitoring during operation and evaluation of the parameters

- Detailed cost monitoring and evaluation

- User surveys (post occupancy evaluation - POE)

- Detailed documentation of the entire project, which is also accessible to external interested parties

With regard to the incentive effect of involvement in a research project, experience shows that building owners and planners see the opportunities that arise from the integration of a project into a research project, but on the other hand there are also concerns about the additional effort that results from this. 


\subsection{Cooperative planning process}

The close cooperation of an experienced planning team has also proven to be beneficial for many of the documented case studies. The good cooperation of the planning team with motivated owners on the one hand and the continuous dialog and coordination with the heritage protection authorities on the other hand proved to be particularly valuable for the success of a project.

Furthermore, the close collaboration between owners and planners / architects with the heritage authority seems to have a clear positive impact on the development of an adequate renovation strategy and the successful implementation of refurbishment measures.

\subsection{Fundamental change of use of buildings}

An important aspect of preserving historic buildings is to increase their economic viability in the long term. Some projects have been able to achieve this with a fundamental change in their use. Private, but especially public owners have spent a lot of energy to realize very special projects that have a significant impact on the surrounding region, mostly in the context of tourism and/or culture (Idrija mercury smelting plant, Ahmet Aga Mansion, solar silo, Musikschule Velden, various farm houses).

\subsection{Other beneficial factors for good practice examples}

Some cases, e.g. buildings owned by the same family over decades or centuries, have shown particularly motivated and dedicated owners, who, beyond a pure consideration of construction costs, also aim at long-term aspects of maintaining the value and adapting the buildings to contemporary uses. This is very clearly documented, for example, in the following videos with "key statements" from owners.

\section{https://www.youtube.com/watch?v=jke7i6muYDs}

\section{https://www.youtube.com/watch?v=fFMq-ijiJ-0}

\section{https://www.youtube.com/watch?v=eAjHbsY8Tq0}

\section{https://www.youtube.com/watch?v=-QCGDXkkvb8}

A central question for building owners is always what good examples are and how to find experienced planners. This is one reason for various case study documentations in recent years, which have been established on a national and international level. Those inventories present good examples which can also be used to establish contact with the planning teams, e.g. Architectural Atlas South Tyrol https://atlas.arch.bz.it/de/

We also found case studies with non-listed historic buildings where the legal obligation for improving the energy performance (when renovating the building) was both starting point and main driver for a comprehensive energy concept that in the end went beyond the legal minimum requirements.

Last but not least, the availability of experienced craftsmen is a key factor for every successful refurbishment project. Some architects have already developed strong and ongoing cooperation with experienced craftsmen and construction companies that are able and willing to deal with the specific requirements of historic buildings. 


\subsection{Overview}

The following table gives a brief overview of the favourable framework conditions that have been identified for the individual case studies. Footnotes below the table give more detailed information on framework conditions in exemplary case studies.

Table 23: Overview on favourable framework conditions

\begin{tabular}{|c|c|c|c|c|c|c|c|}
\hline No & Case study & 胥 & 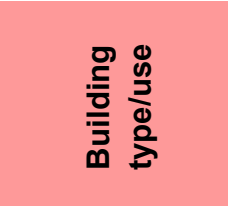 & 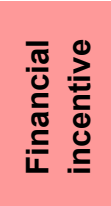 & 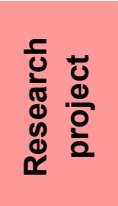 & 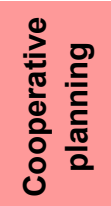 & 㐫 \\
\hline 1 & $\begin{array}{l}\text { Klostergebäude } \\
\text { Kaiserstrasse }\end{array}$ & AT & Res / urban & $x$ & $\mathrm{X} 8$ & $x$ & \\
\hline 2 & Farm house Trins & AT & Res / rural & $x$ & $\mathrm{X} 11$ & & \\
\hline 3 & Hof 6, Schwarzenberg, & AT & Res / rural & & & & $x$ \\
\hline 4 & Kelchalm - Bochumer & AT & Hotel/Rest & $x$ & & & $x$ \\
\hline 5 & Mariahilferstrasse 182 & AT & Res / urban & $x$ & $\mathrm{X} 18$ & $x$ & \\
\hline 6 & House Maurer, Wolfurt & AT & Res / rural & & & & $x$ \\
\hline 7 & $\begin{array}{l}\text { House Breuer, } \\
\text { Tschagguns }\end{array}$ & AT & Res / rural & & & & $x$ \\
\hline 8 & Music school in Velden & AT & Educational & $x$ & $x$ & & $x$ \\
\hline 9 & Hof NeuhäusI & AT & Res / rural & & & & $x$ \\
\hline 10 & Community Hall & AT & Community Hall & $x$ & & & $x$ \\
\hline 11 & Freihof Sulz & AT & Multipurpose & $x$ & $x$ & $x$ & \\
\hline 12 & Oeconomy building & AT & Res + Atelier & & & & $x$ \\
\hline 13 & Giatla Haus & AT & Hotel/Rest & $x$ & & & $x$ \\
\hline 14 & Rhine Valley House & AT & Res / rural & & & & $x$ \\
\hline 15 & $\begin{array}{l}\text { Baur Residence, } \\
\text { Lustenau }\end{array}$ & AT & Res / rural & & & & $x$ \\
\hline 16 & Kasperhof & AT & Res / rural & & & & $x$ \\
\hline 17 & Maison Rubens & $\mathrm{BE}$ & Res / urban & $\mathrm{X} 15$ & & & \\
\hline 18 & Half-timberframed house & $\mathrm{BE}$ & Res / rural & & & $\mathrm{X} 16$ & \\
\hline 19 & Doragno Castle, Rovio & $\mathrm{CH}$ & Res / rural & & & & $x$ \\
\hline 20 & Solar silo & $\mathrm{CH}$ & Offices & & $x$ & $x$ & $\mathrm{X} 14$ \\
\hline 21 & $\begin{array}{l}\text { Wohn- und } \\
\text { Geschäftshaus } \\
\text { Feldbergstraße }\end{array}$ & $\mathrm{CH}$ & Res / urban & $x$ & & & $x$ \\
\hline 22 & $\begin{array}{l}\text { Mehrfamilienhaus } \\
\text { Magnusstrasse }\end{array}$ & $\mathrm{CH}$ & Res / urban & $x$ & & & $x$ \\
\hline 23 & $\begin{array}{l}\text { St. Franziskus Church } \\
\text { Ebmatingen }\end{array}$ & $\mathrm{CH}$ & Religious & & & $x$ & $x$ \\
\hline 24 & $\begin{array}{l}\text { Kindergarten and } \\
\text { apartments (PEB) Chur }\end{array}$ & $\mathrm{CH}$ & Educational & $x$ & & $x$ & $x$ \\
\hline 25 & $\begin{array}{l}\text { Single family home } \\
\text { Luisenstrasse - Bern }\end{array}$ & $\mathrm{CH}$ & Res / urban & & & & $x$ \\
\hline 26 & $\begin{array}{l}\text { Single Family House - } \\
\text { Gstaad }\end{array}$ & $\mathrm{CH}$ & Res / rural & & & & $x$ \\
\hline 27 & Glaserhaus in Affoltern & $\mathrm{CH}$ & Res / rural & & & $x$ & $x$ \\
\hline 28 & PalaCinema Locarno & $\mathrm{CH}$ & Cultural & $x$ & & & $x$ \\
\hline 29 & Casa Rossa Chemnitz & $\mathrm{DE}$ & Res / urban & $x$ & & & $x$ \\
\hline
\end{tabular}




\begin{tabular}{|c|c|c|c|c|c|c|c|}
\hline 30 & Rathaus Bergrheinfeld & DE & Town Hall & & & & $\mathrm{x}$ \\
\hline 31 & Farmhouse Straub & DE & Res / rural & & & & $x$ \\
\hline 32 & Early work Sep Ruf & $\mathrm{DE}$ & Res / rural & & & & $x$ \\
\hline 33 & Ackerbürgerhäuschen & DE & Res / rural & & & & $\mathrm{x}$ \\
\hline 34 & Ritterhof & DE & Res / rural & $X$ & & & $x$ \\
\hline 35 & Rathaus Burgkunstadt & $\mathrm{DE}$ & Townhall & & & & $x$ \\
\hline 36 & Osramhuset (The Osram & DK & Comm Centre & $x$ & & $x$ & $\mathrm{X} 1$ \\
\hline 37 & Klitgaarden & DK & Res / rural & & X9 & & \\
\hline 38 & RYESGADE 30 & DK & Res / urban & $x$ & & & \\
\hline 39 & $\begin{array}{l}\text { Timber-framed house in } \\
\text { Alsace }\end{array}$ & $\mathrm{FR}$ & Res / rural & $x$ & & & $\mathrm{X} 12$ \\
\hline 40 & $\begin{array}{l}\text { Elementary School in } \\
\text { Mulhouse }\end{array}$ & FR & Educational & & & $\mathrm{X} 17$ & \\
\hline 41 & $\begin{array}{l}\text { Timber-framed barn in } \\
\text { the north of France }\end{array}$ & FR & Res / rural & & & & $x$ \\
\hline 42 & Rainhof & IT & Res / rural & & $x$ & $\mathrm{X} 2$ & \\
\hline 43 & Villa Castelli & IT & Res / rural & $\mathrm{X} 3$ & & $x$ & \\
\hline 44 & Ansitz Kofler & IT & Res / urban & & $x$ & $x$ & $X 4$ \\
\hline 45 & $\begin{array}{l}\text { Basilica di Santa Maria di } \\
\text { Collemaqaio }\end{array}$ & IT & Religious & $x$ & $x$ & $x$ & $\mathrm{X} 10$ \\
\hline 46 & House Pernter & IT & Res / rural & & & & $x$ \\
\hline 47 & Kohlerhaus & IT & Res / urban & $\mathrm{X}$ & & & $\mathrm{x}$ \\
\hline 48 & Ruckenzaunerhof & IT & Res / rural & & & & $\mathrm{X} 19$ \\
\hline 49 & Aussergrubhof & IT & Res / rural & & & & $\mathrm{X} 20$ \\
\hline 50 & Oberbergerhof & IT & Res / rural & & & & $\mathrm{X} 21$ \\
\hline 51 & Platzbonhof & IT & Res / rural & & & & $\mathrm{X} 22$ \\
\hline 52 & Mairhof & IT & Res / rural & & & $x$ & $\mathrm{X} 23$ \\
\hline 53 & Obergasserhof & IT & Res / rural & & & $x$ & X24 \\
\hline 54 & Rebecco Farm & IT & B\&B - Farm & $x$ & $x$ & & $x$ \\
\hline 55 & Villa Capodivacca & IT & Res / urban & $x$ & & & \\
\hline 56 & House Moroder & IT & Res / urban & & & & $x$ \\
\hline 57 & Huberhof & IT & Res / rural & $X$ & & & $x$ \\
\hline 58 & Notarjeva vila & SL & Res / urban & $x$ & & & $x$ \\
\hline 59 & Rožna ulica 15, Idrija & SL & Res / urban & $x$ & & & $x$ \\
\hline 60 & Hiša trentarskih & SL & Multipurpose & & & & $x$ \\
\hline 61 & Idrija mercury smelting & SL & Educational & $x$ & & & $x$ \\
\hline 62 & Mercado del Val, & $\mathrm{SP}$ & Retail & $X$ & $\mathrm{X} 13$ & & \\
\hline 63 & Correria 119 & SP & Res / urban & & $x$ & & $x$ \\
\hline 64 & Ahmet Aga Mansion & TK & Offices & & & & $x$ \\
\hline 65 & Nwcip Pasa Library & TK & Library & & & & $x$ \\
\hline 66 & Downie's Cottage & UK & Res / rural & & $\mathrm{X} 7$ & & \\
\hline 67 & Holyrood Park Lodge & UK & Retail & & & & $\mathrm{X} 6$ \\
\hline 68 & Annat Road & UK & Res / urban & & & $x$ & $\mathrm{x}$ \\
\hline 69 & Aspinall Courthouse & USA & Offices & & & $x$ & $x$ \\
\hline
\end{tabular}

1 In connection with the Climate Change Conference (COP 15, 2009) the City of Copenhagen initiated a strategic cooperation with a number of Danish enterprises for the purpose of mutual profiling on climate-friendly buildings. The renovation of the OSRAM Culture Centre was a part of this cooperation and acted as a spearhead for possibilities and methods of renovating old industrial and commercial buildings worth preserving. The project 
received public funding from several institutions and initiatives (Copenhagen Energy Pool, Pool for CO2 neutrality in existing city districts, Urban renewal funds, Accessibility pool).

2 The proposed project was developed in consultation and strong collaboration with the heritage office of the province of Bolzano. The project was the result of a series of meetings and discussions between all the parts involved. The open dialogue and the mutual support between building owner, architect and heritage office ensured the quality of the retrofit, promoted the valorisation of the heritage and reassured the building owner that the design was going to be in coherence with local construction regulations and high standards of quality and comfort in the building.

3 Technical variants for the refurbishment of the building were defined in such a way that the different levels of the casa clima tax bonus can be achieved. The decision was based on a life cycle cost analysis of the different refurbishment options. Source: EURAC Paper Troi et al. (HiBERatlas).

The Italian eco-bonus for the energy upgrade of existing buildings is still in place:

https://www.idealista.it/en/news/financial-advice-italy/2020/01/13/2658-green-building-incentives-italy-how-makeyour-home

4 Building owner Hans Glauber has been active in climate protection for decades and, as president of the Ökoinstitut in Bolzano. As a South Tyrolean visionary in matters of solar energy and climate protection, he wanted to set an example with the refurbishment of his own listed building. The renovation concept was elaborated in close collaboration with the preservation office of South Tyrol.

6 The involved stakeholders (Historic Environment Scotland and the City of Edinburgh Council, Planning Department) represent the public interest to demonstrate that a listed building can be thermally upgraded in a sensitive and proportionate way, improving its performance, yet respecting the existing historic fabric. As it is an accessible site, it has been effective in allowing people to view the measures and understand what can be done in other traditional buildings.

7 Very detailed 40 pages documentation through Historic Environment Scotland is available. https://www.hiberatlas.com/smartedit/projects/32/refurb-case-study-22.pdf

8 The project was part of the national R\&D programme "City of the Future" and received funding for the implementation of innovative technical measures, monitoring and detailed documentation. Furthermore, the project received funding from the wohnfonds Vienna for "normal" urban renewal and refurbishment measures. Project and monitoring reports are available (in German) for download:

https://nachhaltigwirtschaften.at/de/hdz/projekte/gruenderzeit-mit-zukunft-demonstrationsprojekt-3-ka-7kaiserstrasse-innovative-sanierung-eines-denkmalgeschuetzten-gruenderzeitgebaeudes-mitinnendaemmung.php

9 The project was also part of the H2020 REBuild-Project and is well documented. Deliverables are available for download: https://www.hiberatlas.com/smartedit/projects/37/RIBuild D3.2 v1.0 1.pdf

10 The case of the Basilica Santa Maria di Collemaggio seems to be a special one in several aspects: The restoration was caused by the earthquake in that struck L'Aquila in 2009. The restoration project involved specific research resources and techniques from Politecnico di Milano (advanced survey, modelling and design techniques, including laser scanning and HBIM). Another special feature of this project is the financing by a private sponsor (ENI).

11 The detailed project description provides some hints on subsidies. University Innsbruck was closely involved in this project (PHPP calculation, hygrothermal assessment, detailed documentation).

https://www.hiberatlas.com/smartedit/projects/40/Bauernhof\%20Trins Projektbeschreibung Fotodokumentation.p $\underline{\mathrm{df}}$

12 The main driver for the implementation of this project seems to be the owner who is involved in a heritage protection association and "spared no expense on the works". The owner often organises open house day for its members. Because the owner wanted to apply to a grant for exterior works related to heritage, a validation on these works by the Alsatian architectural review board was required. The house is one of the case studies of the CREBA (French knowledge center for responsible retrofit of heritage building) website. http://www.rehabilitationbati-ancien.fr/fr/retours-d-experiences/une-rehabilitation-energetique-et-une-restauration-patrimoniale-exemplaire 
13 R\&D companies were involved within the CommONEergy project. The total intervention cost was covered with different sources: The European Commission provided a grant through the European Project "CommONEnergy". Public funds from the Valladolid City Council and Junta de Castilla y León. Private funds from the owners of the stalls.

14 Solar Silo provides an exceptional example of private investors getting involved into the transformation of an old industrial site into an area for cultural, social and commercial uses. After Kantensprung AG was able to attract private investors to contribute one-fifth of the purchase price for the site, the Abendrot Foundation used its network of contacts to convince two other institutional investors, the Social Fund Foundation and the Basel Pension Fund, as well as a private investor, to join the project. Furthermore, the project was developed in close cooperation with the department for building conservation and with scientific partners (FHNW University of Applied Sciences and Arts Northwestern Switzerland / Muttenz).

15 Involvement of Bruxelles environnement: The owners have received subsidies for the total renovation "energy premium and renovation premium" from the government. 100euros $/ \mathrm{m}^{2}$ have been subsided by Brussels environment (appr. 10\% of total investment costs).

16 Close cooperation between Flanders Heritage Agency, Antea Belgium nv (Research) architect, conservation consultant and energy consultant.

17 Solutions that are acceptable to all have been found through dialogue between the contracting authority, the project management team and the teams of the Regional Directorate for Cultural Affairs (DRAC).

18 The project was part of the national R\&D programme "City of the Future" and received funding for the implementation of innovative technical measures, monitoring and detailed documentation. Furthermore, the project received funding from the wohnfonds Vienna for "normal" urban renewal and refurbishment measures. Project and monitoring reports are available (in German) for download:

https://nachhaltigwirtschaften.at/en/sdz/projects/enerphit-green.php

19 For the whole family, the construction phase was like a voyage of discovery through the building, through which they learned a lot about past times and the history of the place. It is therefore a great concern for all of them that the building is preserved for future generations and that the knowledge they have gained is passed on.

20 The owner is a qualified master carpenter and wanted to preserve the built heritage of the previous generations, also in the course of the reconstruction and expansion of the living space. "It was important to me to treat what my ancestors had built with heart."

21 The renovation of the Oberbergerhof should accommodate the home of the younger farmer and his family, as well as providing accommodation for the harvest workers. First of all, it was necessary to create a separate entrance for the young family, so that every generation could keep a certain degree of privacy in the shared apartment building.

22 Legal obligation: Since the house is not a listed building and is therefore subject to the obligation of energetic renovation, among other things the old plaster had to be removed, which was replaced by an insulating plaster.

23 During the refurbishment process, the owner got to know his building down to the last detail because he himself was looking for practical solutions. In doing so, he did his own research and was always in contact with the monument authority and the architect, so that an optimal solution could be found through cooperation. A compromise was always sought between the requirements of monument protection and a practical, efficient solution. It is remarkable that the client himself determined what he considered worthy of protection. The architect then served as a liaison with the Office for the Protection of Monuments. It should also be emphasised that such a comprehensive refurbishment requires professional craftsmen with a great deal of experience and competence.

24 The conditions for success of this project were the intensive exchange with the monument authority. In addition, expert and local craftsmen proved their worth in the implementation of the measures. 


\subsection{Conclusions from the planning process}

Here are some examples: of the most valuable insights relating to the overall planning process:

- Complex projects require an ambitious planning team and an open-minded client, as well as a continuous process of coordination between planners and heritage authorities (Klostergebäude Kaiserstrasse)

- Informal cooperation with heritage authorities in the early stage pays back later on in the project (Glaserhaus)

- Close cooperation within the planning team and with the users is essential for the success of complex projects (Klostergebäude Kaiserstrasse, Musikschule Velden)

- Private owners are not optimal for testing new methods, as they might have strong ideas about what to do from the outset. (Alken)

- The budget is decisive for which measures that are implemented in the end. (Alken)

- Despite a tight budget and a complex project, acceptable solutions were found through dialogue with owner, planning team and heritage authorities (Cour de Lorraine)

- The owner had to convince many people that the project was at all possible, without being persistent it had not been possible to realise it. (Timber-framed house in Alsace)

- Costs for innovative materials should be calculated early in the project, to avoid unnecessary planning efforts. (Villa Castelli)

- An engaged and knowledgeable owner can contribute with practical solutions customized to the building (Mairhof)

- It can take time and effort to make new and innovative building components to work in practice. (Solar silo)

- Creating a positive mindset among all involved project partners early on in the project can be a success factor when solving complex problems that require cooperation (Freihof Sulz)

- It is important to involve the future facility managers of the building in the project already during the planning phase. This is often problematic due to a lack of personnel, especially in public buildings. (Musikschule Velden)

- Collaboration with local craftspeople can contribute to the preservation of local crafts and local architectural features. (Giatla House)

- The planning authorities declining initial standard solutions can lead to a process where innovative solutions are developed (Single family House - Bern)

- A detailed survey of the building is essential for adequate planning (Haus Pernter)

- Public-private partnership, together with the experts' vision of the retrofit was a winning strategy: it made possible to combine solutions in a well-calibrated system of activities, with a long-term vision of the building management. The participation of some farms, trainers, small local - and extra-local innovators has been very good for defining the functions and the needs of the building. (Rebecco farm)

From these learnings, the following overarching conclusions can be drawn from the perspective of the planning process:

- An early and iterative dialogue between the planning team and the heritage authorities is in many ways a key to a successful end result and to an efficient planning process.

- Engaged clients can contribute to the project in many different ways, from identifying what is worthy to preserve to customized technical solutions and lead to an "ownership" of the project. Suggestions can be made by the planning team, but a lot depends on the budget of the owners with little influence for expert team.

- Expert dialogue is important, but the involvement of local craftsmen and companies, who have experience in the region, is valuable for the planning process as well.

- Limited project budgets can hamper innovative solutions that are cost efficient over the whole life cycle.

- A comprehensive understanding of the building is essential for identifying good solutions. 


\subsection{Conclusions from the refurbishment measures}

Most information collected regarding refurbishment measures is from walls. It would be obvious to say this is due to the historic character of the buildings and consequently the need for adapting the structure of the walls to the state of the art. Nevertheless, this cannot be concluded from the HiBERatlas as there was no information collected to specify this reason. The most applied measure regarding walls is adding internal insulation (41 of 69 cases) thus being able to keep the visual perception of the buildings from the outside. Specifically, capillary active materials ( 28 cases) and vapour retardant layers are used to make the walls more energy efficient. Regarding materials, there is a trend towards ecological materials like hemp fiber, wood fiber and cellulose fiber. External insulation is mostly just used for unlisted buildings.

Roofs were often in bad shape; therefore $\mathbf{4 5}$ case studies have refurbished their roofs. Mostly this refers to insulation between rafters of pitched roofs. As for materials, wood fibre is the most commonly used solution. With these measures a huge improvement of U-values could be achieved. It needs to be said, that 22 roofs had to be completely renovated but they tried to keep the shape and the materials of the original one.

The ground floors were refurbished in $\mathbf{4 5}$ case studies, 20 of these with completely new concrete slabs and insulation. Here, many possibilities exist to reconcile energetic necessity and monument protection as the primarily important aspect are the preservation of the floor coverings which can usually be added after renewing the sub-structure.

Regarding the windows, 67 different solutions were documented. The overall similarity being that there are significant increases of energy efficiency in most cases. This is very often due to the exchanging of singlelayer glazing to double or triple layer glazing. Nevertheless, also in $\mathbf{4 5}$ cases windows were replaced, 13 of these has no heritage value restrictions and documented "off the shelf solutions", but also 24 had handcrafted new solutions keeping the appearance of the building.

For the integration of renewables (22 solar, 19, PV, 10 geothermal, 25 biomass) there is too little data on each case study to derive conclusions. The most obvious one is, that there are more interventions in rural areas.

For HVAC, there are $\mathbf{2 8}$ cases with new heating solutions with biomass. Domestic hot water solutions were not changed in 18 cases, but 40 document a combination with heating. Interesting is the implementation of ventilation systems in 35 cases along with frequent adaption of natural ventilation. The mechanical ventilation systems mostly come with heat recovery, 26 of them are designed as central systems. There is a clear sensibility for indoor air quality - especially in the education sector - no matter how old the building is. This seems to be i.a. due to the importance for long-term preservation of the historic buildings.

In general, one can say that with refurbishment measures of historical buildings nothing is the norm and everything is special. Still there are similarities in some projects, e.g. sometimes due to the location in the same geographical area, sometimes due to the same building type.

\subsection{Conclusions from evaluation and outcomes}

Once implemented, the energy efficient measures have to demonstrate their performance in real life conditions. Looking back "ex-post" helps future implementations, both re-assuring that targets were reached with qualitative and quantitative assessments and pointing out possibly weak points.

This report includes 69 case studies in the assessment, however the number of projects that include detailed information about the evaluation of the intervention is still sometimes limited. It would be interesting to know the reasons for the lack of documentation, e.g. financing especially the require personnel resources for documentation or issues with privacy regulations -as these were aspects registered within the project. There is also the question of the documentation of products, which failed their intent. Naturally, there is little to no information on these, although learnings about the reason why they failed could improve future development of measure. Generally, if there are no official obligations, the average owner will not evaluate energy efficiency measure, amongst others due to financial reasons.

Due to the limited information, any analysis presented here must be taken carefully. While comprehensive energy, comfort monitoring, and POEs (post occupancy evaluations) are usually mostly available for renovations included in research projects in the case studies analysed in the HiBERatlas, a qualitative feedback from the 
occupants on the reached indoor climate is provided in the majority of cases, e.g. orally in interviews with building owners but also guests and tenants, thus not just those who had taken the decisions.

A first remark should therefore be made regarding the difficulties in gathering this information. It would be important to identify the reasons for the limited number of answers and depth of the information gathered so far. A first step in this direction could be to ask directly to the authors that documented these projects in the database.

Of the four sections that form the "Evaluation" chapter (chapter 5), energy efficiency is by far the best documented part. This is probably due to policies implemented at regional or national level that made these calculations compulsory. Even in the cases where listed buildings or energy renovations are exempted, several projects have included these calculations. On the other hand, on-site monitoring of buildings' performance is still very rare and seems to be limited mostly to research related projects.

The level of information gathered on the energy use of the buildings, together with the detailed description of the technical aspects of the building and the retrofit, have allowed a further evaluation of the results looking at the entire sample. In general, it is worth noticing the important reduction in energy use independently of the type of use or level of protection of the building. The number of case studies documented within the climatic zone Cfb even allowed an analysis of the cases with comparable climatic conditions. The analysis however cannot be extended to other climates until the sample size has increased considerably. In any case, it was observed that the external (climate) and intrinsic (building age, typology, size) variables of the case studies have a much larger impact on the energy demand before the intervention than afterwards.

Regarding the documentation of the internal climate, comments from users on the achieved thermal comfort are recorded in most cases and often underlining the improvement. With answers in around two thirds of the cases, indoor air quality seems to be a bit less immediate to be assessed by the occupants. Answers include both comments on the ventilation strategy, the deliberate decision for natural ventilation or the satisfaction with a controlled ventilation system, and in a few cases also issues like annoying noise but also awareness that manual ventilation is needed both for good air quality and to avoid mould. Answers on daylight and acoustic comfort are given in less than half of the cases, the first sometimes mentioning that small windows limit of course the daylight.

There are a few cases with full POEs included in the HiBERatlas, the most comprehensive perhaps Ansitz Kofler (Italy), which embraces a monitoring of (i) energy consumption, (ii) indoor comfort and (iii) hygrothermal performance (of three kinds of insulation systems - interior with mineral wool, interior with wood fibre and exterior - as well as temperatures at thermal bridges). The latter confirms calculated values, both the calculated U-values and the moisture levels within the wall staying low enough. Indoor comfort was verified both for winter and summer conditions and the difference in energy consumption with around $50 \mathrm{kWh} / \mathrm{m}^{2} \mathrm{y}$ compared to calculated $30 \mathrm{kWh} / \mathrm{m}^{2} \mathrm{y}$ could be explained partly by tenants using the shading for privacy and thus reducing solar gains (showing that once the energy balance goes towards zero, the influence of user behaviour becomes crucial). A higher energy consumption was also reported for Ryesgade 30 (Denmark), where the $83 \mathrm{kWh} / \mathrm{m}^{2} \mathrm{a}$ compared to expected 56 $\mathrm{kWh} / \mathrm{m}^{2} \mathrm{a}$ was attributed to higher indoor temperature of $22-23^{\circ} \mathrm{C}$ (compared to $20^{\circ} \mathrm{C}$ as assumed) chosen by tenants, which results in $53 \%$ savings rather than $63 \%$ (also known as rebound effect). That the measured demand can, however, also be lower than calculated is shown in the Glaserhaus in Affoltern (Switzerland), where the consumption in the monitored period was actually $10 \%$ lower than expected.

Although financial aspects are often highlighted as both triggers (reducing running costs) and limitations (excessive investment costs) for carrying an energy renovation, the projects documented so far have included very limited information. An effort should be made in improving this section, as this could play a crucial role in the relevance and success of the database among private owners.

Analogously, the information regarding the environmental performance of the building after the retrofit is very scarce, especially when looking at LCA and water management.

\subsection{Conclusions regarding the favourable framework conditions and transferability}

In many of the examples we can observe the interaction and overlapping of several favourable framework conditions: e.g. involvement in a research project that goes hand in hand with financial support for innovative measures and where at the same time a committed and experienced planning team works together from the very beginning of the process. (Alken) 
In addition to the classic financial incentive models (tax reductions, subsidies, soft loans), there are also interesting alternative approaches such as sponsoring by private companies and private investors, which have made it possible in one case to transform a former industrial site into a multifunctional area (Solar Silo).

The public sector can play a very important role as owner when it comes to exemplary redevelopment and the development of innovative projects that are also open to the public (Osramhuset, Holyrood Lodge, Ahmet Aga Mansion).

To ensure long-term economic viability, the fundamental change of use of a building can sometimes make an important contribution to preserve historic buildings if this change is adapted to the technical possibilities of the building and with respect for the heritage values (Idrija mercury smelting plant, Ahmet Aga Mansion, solar silo, Musikschule Velden, various farm houses). This has to be done in a sensible way, as it can also lead to the building not being functional for the new use. It is recommended to first consider the limited characteristic of the building and the past use before suggesting to fundamentally change the use of the building. One has to consider, that in HiBERatlas, the case studies with a fundamental change of use are best-practice examples, most of them adapted during a research context and better documented than usual projects

A key moment in the life-cycle of Alpine farmhouses is the change in ownership (in some cases a generational change). Generally, owners play a central role in private housing, but at this special point often important decisions are made on how to deal with historic buildings.

Some of the above-mentioned favourable framework conditions can also serve as a model for other countries and have considerable potential for transferability. In particular, these are national research programmes that focus on the high-quality renovation of historic buildings and thus enable the implementation, but also the documentation and detailed monitoring of demonstration projects. In addition, national or regional authorities should also provide targeted financial incentives to make better use of the possibilities for energy-efficient renovation of historic buildings.

Overall, the HiBERatlas has collected a vast amount of case studies for the renovation of historical buildings towards zero energy, which will be the source of inspiration and expertise for clients, planners, experts, craftsmen, scientists and students and others for future renovations of historical buildings. Nevertheless, we would like to point out, that one could additionally gather case studies were everything failed to see what can happen and to learn from them. 